\title{
Atypical Dearomative Spirocyclization of $\beta$-Naphthols with Diazoacetamides using a Silver Catalyst
}

\section{Haruka Homma, ${ }^{\dagger}$ Shingo Harada ${ }^{*}, \dagger$ Tsubasa Ito, ${ }^{\dagger}$ and Ayaka Kanda, Tetsuhiro Nemoto*,t,‡}

† Graduate School of Pharmaceutical Sciences, Chiba University, 1-8-1, Inohana, Chuo-ku, Chiba 260-8675 (Japan)

Tel/Fax:81-43-226-2920.

E-mail:tnemoto@faculty.chiba-u.jp, Sharada@chiba-u.jp

¥Molecular Chirality Research Center, Chiba University, 1-33, Yayoi-cho, Inageku, Chiba 263-8522 (Japan)

\section{Table of Contents}

1. General Information. .S2

2. Characterization of Dearomatized Products 4a-4i, 5a, 6a, 7c, 8c, 9a-9l, 10a.....S3

3. X-ray Crystallographic Data. S24

4. Additional Studies on $\mathrm{F}^{-}$Anion Sensor. S31

5. Synthesis and Characterization of Substrates.....................................S34

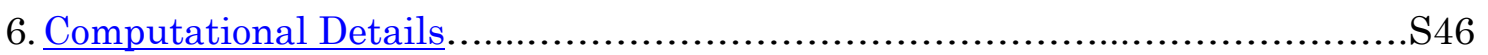

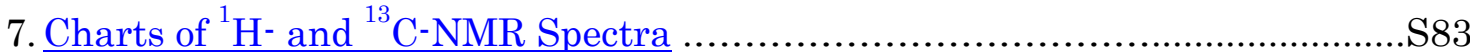

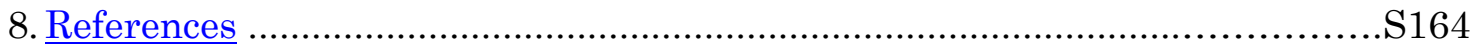




\section{General Information}

NMR spectra were recorded at $400 \mathrm{MHz}$ or $600 \mathrm{MHz}$ for ${ }^{1} \mathrm{H} \mathrm{NMR}, 100 \mathrm{MHz}$ or $150 \mathrm{MHz}$ for ${ }^{13} \mathrm{C} \mathrm{NMR}$, and $564 \mathrm{MHz}$ for ${ }^{19} \mathrm{~F} \mathrm{NMR}$ Chemical shifts in $\mathrm{CDCl}_{3}$, $\mathrm{CD}_{3} \mathrm{OD}$, or DMSO-d6, were reported downfield from TMS (=0 ppm) or solvent signals $\left[\mathrm{CH}_{3} \mathrm{OH}(3.31 \mathrm{ppm})\right.$, DMSO (2.50 ppm)] for ${ }^{1} \mathrm{H}$ NMR. Data are reported as follows: chemical shift, multiplicity $(\mathrm{s}=$ singlet, $\mathrm{d}=$ doublet, $\mathrm{t}=$ triplet, sep = septet, $\mathrm{m}=$ multiplet, and $\mathrm{br}=$ broad $)$, integration and coupling constants in $\mathrm{Hz}$. For ${ }^{13} \mathrm{C} \mathrm{NMR}$, chemical shifts were reported in the scale relative to the solvent signal $\left[\mathrm{CHCl}_{3}(77.16 \mathrm{ppm}), \mathrm{CH}_{3} \mathrm{OH}(49.00 \mathrm{ppm}), \mathrm{DMSO}\right.$ (39.52 ppm)] as an internal reference. ESI mass spectra were measured on JEOL AccuTOF LC-plus JMS-T100LP. Optical rotations were measured on a JASCO P-1020 polarimeter. Melting points were measured with a SIBATA NEL-270 melting point apparatus. The UV-Vis spectrometer was a JASCO V730 spectrometer. Analytical thin layer chromatography was performed on Kieselgel 60F254, $0.25 \mathrm{~mm}$-thick plates. Column chromatography was performed with silica gel $60 \mathrm{~N}$ (spherical, neutral 63-210 mesh). Reactions were conducted in dry solvent. Other reagents were purified by the usual methods. 


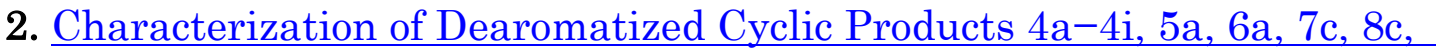
9a-91, 10a

\section{General Procedure A for Reductive Dearomatization}

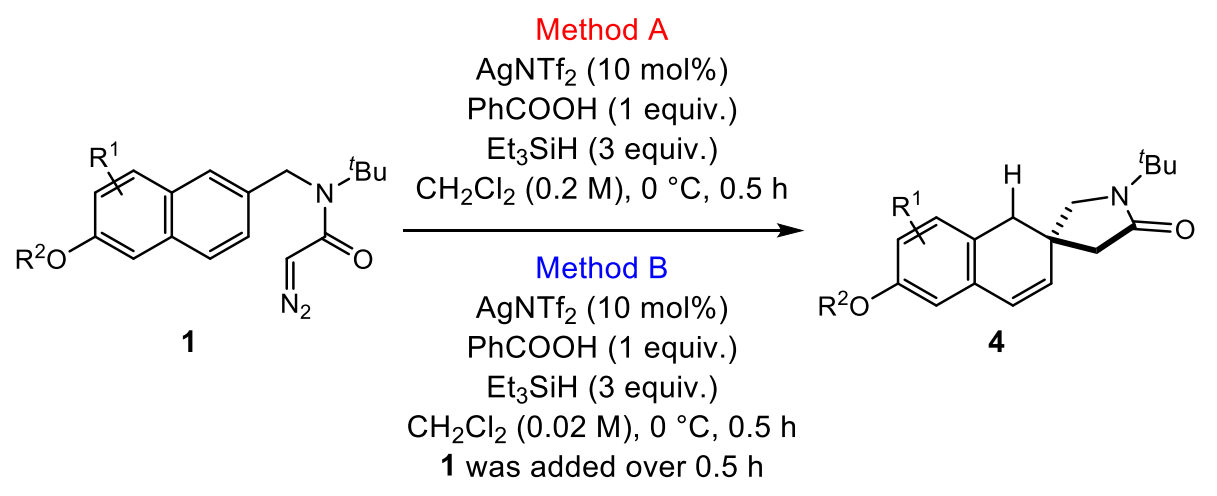

Method A: A pre-dried $30 \mathrm{~mL}$ eggplant-shaped flask equipped with a magnetic stir bar was charged with $\mathrm{AgNTf}_{2}(3.9 \mathrm{mg}, 0.01 \mathrm{mmol}, 10 \mathrm{~mol} \%)$, benzoic acid (12.2 mg, $0.1 \mathrm{mmol}, 1$ equiv.), and triethylsilane ( $47.9 \mu \mathrm{L}, 0.3 \mathrm{mmol}, 3$ equiv.), which were subsequently dissolved partially in $\mathrm{CH}_{2} \mathrm{Cl}_{2}(0.5 \mathrm{~mL})$ under an argon gas atmosphere. Diazo compound 1 (0.1 mmol) was added to the suspension at $0{ }^{\circ} \mathrm{C}$ in an ice bath, and the reaction mixture was stirred for 0.5 $\mathrm{h}$ at the same temperature. The reaction was quenched with the addition of water and $1 \mathrm{M}$ TBAF in THF $(0.1 \mathrm{~mL})$, and stirred for $15 \mathrm{~min}$ at room temperature. The aqueous solution was extracted with $\mathrm{CH}_{2} \mathrm{Cl}_{2} \times 2$. The combined organic layers were washed with brine, dried over $\mathrm{Na}_{2} \mathrm{SO}_{4}$, and concentrated under reduced pressure. The obtained crude residue was purified by flash column chromatography (column condition; gradient elution: $n$-hexane/EtOAc, $3 / 1 \rightarrow 1 / 1$ ) to afford spirocyclic compound 4 .

Method B: A pre-dried $30 \mathrm{~mL}$ eggplant-shaped flask equipped with a magnetic stir bar was charged with $\mathrm{AgNTf}_{2}(3.9 \mathrm{mg}, 0.01 \mathrm{mmol}, 10 \mathrm{~mol} \%)$, benzoic acid (12.2 mg, $0.1 \mathrm{mmol}, 1$ equiv.), and triethylsilane ( $47.9 \mu \mathrm{L}, 0.3 \mathrm{mmol}, 3$ equiv.), which were subsequently dissolved partially in $\mathrm{CH}_{2} \mathrm{Cl}_{2}(1 \mathrm{~mL})$ under an argon gas atmosphere. Diazo compound $1(0.1 \mathrm{mmol})$ in $\mathrm{CH}_{2} \mathrm{Cl}_{2}(4 \mathrm{~mL})$ was added slowly over $0.5 \mathrm{~h}$ via a syringe pump to the suspension at $0{ }^{\circ} \mathrm{C}$ in an ice bath, and the reaction mixture was stirred for $0.5 \mathrm{~h}$ at the same temperature. The reaction was quenched with the addition of water and $1 \mathrm{M}$ TBAF in THF (0.1 
$\mathrm{mL}$ ), and stirred for $15 \mathrm{~min}$ at room temperature. The aqueous solution was extracted with $\mathrm{CH}_{2} \mathrm{Cl}_{2} \times 2$. The combined organic layers were washed with brine, dried over $\mathrm{Na}_{2} \mathrm{SO}_{4}$, and concentrated under reduced pressure. The obtained crude residue was purified by flash column chromatography (column condition; gradient elution: $n$-hexane/EtOAc, $3 / 1 \rightarrow 1 / 1$ ) to afford spirocyclic compound 4.

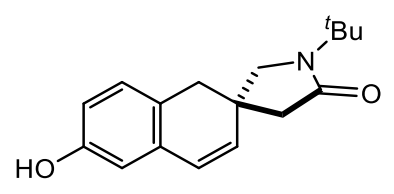

\section{1'-(tert-Butyl)-6-hydroxy-1H-spiro[naphthalene-2,3'-pyrrolidin]-5'-one (4a)}

Prepared according to the general procedure A (Method A) using 1a (29.7 mg), and isolated as white solid (21.6 mg, 80\% yield). m.p. $220-221^{\circ} \mathrm{C} ; \mathrm{R}_{f}=0.4\left(n^{-}\right.$ hexane/EtOAc, 1/1); ${ }^{1} \mathrm{H} \mathrm{NMR}\left(600 \mathrm{MHz} \mathrm{CDCl}_{3}\right) \delta 6.95(\mathrm{~d}, J=9.6 \mathrm{~Hz}, 1 \mathrm{H})$, $6.63(\mathrm{~d}, J=9.6 \mathrm{~Hz}, 1 \mathrm{H}), 6.58(\mathrm{~s}, 1 \mathrm{H}), 6.39(\mathrm{~d}, J=9.6 \mathrm{~Hz}, 1 \mathrm{H}), 5.86(\mathrm{~d}, J=9.6$ $\mathrm{Hz}, 1 \mathrm{H}), 5.67$ (br s, $1 \mathrm{H}), 3.37$ (d, $J=10.8 \mathrm{~Hz}, 1 \mathrm{H}), 3.30(\mathrm{~d}, J=10.8 \mathrm{~Hz}, 1 \mathrm{H})$, $2.81(\mathrm{~d}, J=15.6 \mathrm{~Hz}, 1 \mathrm{H}), 2.77(\mathrm{~d}, J=15.6 \mathrm{~Hz}, 1 \mathrm{H}), 2.38(\mathrm{~d}, J=18.6 \mathrm{~Hz}, 1 \mathrm{H})$, $2.35(\mathrm{~d}, J=18.6 \mathrm{~Hz}, 1 \mathrm{H}), 1.39(\mathrm{~s}, 9 \mathrm{H}) ;{ }^{13} \mathrm{C} \mathrm{NMR}\left(150 \mathrm{MHz}, \mathrm{CDCl}_{3}\right) \delta 174.1$, $155.1,134.9,134.3,129.4,128.5,124.9,114.2$, 113.5, 55.8, 54.2, 45.5, 38.6, 36.8, 27.9 (3C); IR (ATR) 3113, 2970, 2928, 1730, 1650, 1601, 1445, 1421, 1365, 1284, $1218 \mathrm{~cm}^{-1}$; HRMS (ESI-TOF) $[\mathrm{M}+\mathrm{Na}]^{+}$calcd for $\mathrm{C}_{17} \mathrm{H}_{21} \mathrm{NNaO}_{2}{ }^{+} \mathrm{m} / z$ 294.1465 , found $\mathrm{m} / \mathrm{z} 294.1465$.

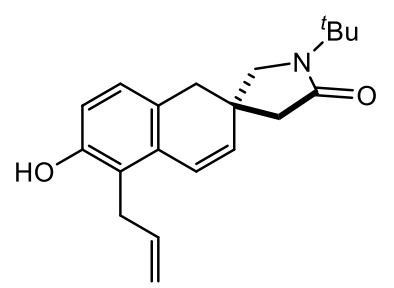

\section{5-Allyl-1'-(tert-butyl)-6-hydroxy-1H-spiro[naphthalene-2,3'-pyrrolidin]-5'- one (4b)}

Prepared according to the general procedure A (Method B) using $\mathbf{1 b}$ (33.7 $\mathrm{mg}$ ), and isolated as yellow solid $(24.3 \mathrm{mg}, 78 \%$ yield $)$. m.p. $69-71{ }^{\circ} \mathrm{C} ; \mathrm{R}_{f}=0.5\left(n^{-}\right.$ hexane/EtOAc, 1/1); ${ }^{1} \mathrm{H} \mathrm{NMR}\left(600 \mathrm{MHz}^{\mathrm{CDCl}} \mathrm{CDC}_{3}\right) \delta .84(\mathrm{~d}, J=7.8 \mathrm{~Hz}, 1 \mathrm{H})$, 
$6.66(\mathrm{~d}, J=9.6 \mathrm{~Hz}, 1 \mathrm{H}), 6.65(\mathrm{~d}, J=7.8 \mathrm{~Hz}, 1 \mathrm{H}), 5.95(\mathrm{ddt}, J=16.8,9.6,6.0$ $\mathrm{Hz}, 1 \mathrm{H}), 5.88(\mathrm{~d}, J=9.6 \mathrm{~Hz}, 1 \mathrm{H}), 5.01(\mathrm{dd}, J=9.6,1.8 \mathrm{~Hz}, 1 \mathrm{H}), 4.92(\mathrm{dd}, J=$ 16.8, $1.8 \mathrm{~Hz}, 1 \mathrm{H}), 3.47(\mathrm{~d}, J=6.0 \mathrm{~Hz}, 2 \mathrm{H}), 3.37(\mathrm{~d}, J=10.2 \mathrm{~Hz}, 1 \mathrm{H}), 3.31(\mathrm{~d}, J$ $=10.2 \mathrm{~Hz}, 1 \mathrm{H}), 2.79(\mathrm{~d}, J=15.6 \mathrm{~Hz}, 1 \mathrm{H}), 2.75(\mathrm{~d}, J=15.6 \mathrm{~Hz}, 1 \mathrm{H}), 2.36(\mathrm{~d}, J$ $=16.8 \mathrm{~Hz}, 1 \mathrm{H}), 2.33(\mathrm{~d}, J=16.8 \mathrm{~Hz}, 1 \mathrm{H}), 1.39(\mathrm{~s}, 9 \mathrm{H}) ;{ }^{13} \mathrm{C}$ NMR $(150 \mathrm{MHz}$, $\left.\mathrm{CDCl}_{3}\right) \delta 174.4,153.6,136.4,134.8,132.3,127.3,125.6,125.0,122.0,115.3$, 114.2, 55.9, 54.3, 44.9, 39.6, 36.1, 29.6, 27.9 (3C); IR (ATR) 3275, 2971, 2926, 2876, 1739, 1649, 1365, $1217 \mathrm{~cm}^{-1}$; HRMS (ESI-TOF) $[\mathrm{M}+\mathrm{Na}]^{+}$calcd for $\mathrm{C}_{20} \mathrm{H}_{25} \mathrm{NNaO}_{2}{ }^{+} \mathrm{m} / z 334.1778$, found $\mathrm{m} / z 334.1770$.

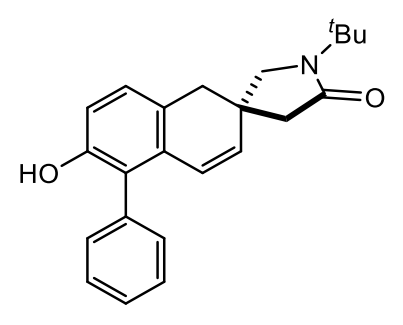

\section{1'-(tert-Butyl)-6-hydroxy-5-phenyl-1 $H$-spiro[naphthalene-2,3'-pyrrolidin]-5'- one (4c)}

Prepared according to the general procedure A (Method B) using 1c $(37.3 \mathrm{mg})$, and isolated as yellow solid (25.4 mg, $73 \%$ yield).: m.p. $225-227^{\circ} \mathrm{C} ; \mathrm{R}_{f}=0.4$ ( $n$-hexane/EtOAc, 2/1); ${ }^{1} \mathrm{H} \mathrm{NMR}\left(600 \mathrm{MHz}, \mathrm{CDCl}_{3}\right) \delta 7.50$ (dd, $J=8.4,8.4 \mathrm{~Hz}$, 2H), $7.43(\mathrm{dd}, J=8.4,8.4 \mathrm{~Hz}, 1 \mathrm{H}), 7.29(\mathrm{~d}, J=8.4 \mathrm{~Hz}, 2 \mathrm{H}), 7.03(\mathrm{~d}, J=9.6 \mathrm{~Hz}$, $1 \mathrm{H}), 6.83(\mathrm{~d}, J=9.6 \mathrm{~Hz}, 1 \mathrm{H}), 6.16(\mathrm{~d}, J=10.8 \mathrm{~Hz}, 1 \mathrm{H}), 5.76(\mathrm{~d}, J=10.8 \mathrm{~Hz}$, $1 \mathrm{H}), 5.26(\mathrm{br} \mathrm{s}, 1 \mathrm{H}), 3.41(\mathrm{~d}, J=10.8 \mathrm{~Hz}, 1 \mathrm{H}), 3.28(\mathrm{~d}, J=10.8 \mathrm{~Hz}, 1 \mathrm{H}), 2.87$ $(\mathrm{d}, J=15.6 \mathrm{~Hz}, 1 \mathrm{H}), 2.84(\mathrm{~d}, J=15.6 \mathrm{~Hz}, 1 \mathrm{H}), 2.39(\mathrm{~s}, 2 \mathrm{H}), 1.39(\mathrm{~s}, 9 \mathrm{H}) ;{ }^{13} \mathrm{C}$ $\mathrm{NMR}\left(150 \mathrm{MHz}, \mathrm{CDCl}_{3}\right) \delta$ 174.0, 152.1, 135.0, 134.3, 131.7, 130.9, 130.8, 129.4, 129.3, 128.8, 128.4, 126.3, 125.6, 125.1, 114.0, 55.5, 54.1, 45.6, 39.3, 36.1, 27.9 (3C); IR (ATR) 3030, 2970, 2934, 2710, 1745, 1644, 1568, 1432, 1364, 1279, $1215 \mathrm{~cm}^{-1}$; HRMS (ESI-TOF) $[\mathrm{M}+\mathrm{Na}]^{+}$calcd for $\mathrm{C}_{23} \mathrm{H}_{25} \mathrm{NNaO}_{2}{ }^{+} \mathrm{m} / z 370.1778$, found $\mathrm{m} / \mathrm{z} 370.1778$. 


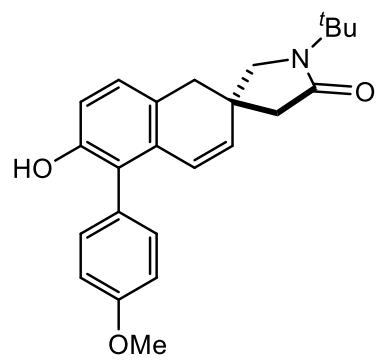

1'-(tert-Butyl)-6-hydroxy-5-(4-methoxyphenyl)-1H-spiro[naphthalene-2,3'pyrrolidin]-5'-one (4d)

Prepared according to the general procedure A (Method B) using 1d (40.3 mg), and isolated as yellow solid (28.5 mg, 76\% yield).: m.p. $199-201{ }^{\circ} \mathrm{C} ; \mathrm{R}_{f}=0.4$ ( $n$-hexane/EtOAc, $1 / 1)$; ${ }^{1} \mathrm{H} \mathrm{NMR}\left(600 \mathrm{MHz} \mathrm{CDCl}_{3}\right) \delta 7.21(\mathrm{~d}, J=9.0 \mathrm{~Hz}, 2 \mathrm{H})$, $7.04(\mathrm{~d}, J=9.0 \mathrm{~Hz}, 2 \mathrm{H}), 7.01(\mathrm{~d}, J=7.8 \mathrm{~Hz}, 1 \mathrm{H}), 6.81(\mathrm{~d}, J=7.8 \mathrm{~Hz}, 1 \mathrm{H}), 6.18$ $(\mathrm{d}, J=9.6 \mathrm{~Hz}, 1 \mathrm{H}), 5.77(\mathrm{~d}, J=9.6 \mathrm{~Hz}, 1 \mathrm{H}), 5.04$ (br s, $1 \mathrm{H}), 3.87$ (s, 3H), 3.40 $(\mathrm{d}, J=10.8 \mathrm{~Hz}, 1 \mathrm{H}), 3.27(\mathrm{~d}, J=10.8 \mathrm{~Hz}, 1 \mathrm{H}), 2.86(\mathrm{~d}, J=15.6 \mathrm{~Hz}, 1 \mathrm{H}), 2.83$ $(\mathrm{d}, J=15.6 \mathrm{~Hz}, 1 \mathrm{H}), 2.37(\mathrm{~s}, 2 \mathrm{H}), 1.38(\mathrm{~s}, 9 \mathrm{H}) ;{ }^{13} \mathrm{C} \mathrm{NMR}\left(150 \mathrm{MHz}, \mathrm{CDCl}_{3}\right) \delta$ 173.9, 159.7, 152.3, 135.0, 135.0, 132.1, 132.0, 128.6, 126.4, 125.9, 125.2, 125.1, 114.9, 114.8, 113.8, 55.5, 55.5, 54.1, 45.7, 39.4, 36.1, 27.9 (3C); IR (ATR) 2956, 2829, 1749, 1648, 1601, 1514, 1444, 1430, 1364, 1281, 1243, $1218 \mathrm{~cm}^{-1}$; HRMS (ESI-TOF) $[\mathrm{M}+\mathrm{H}]^{+}$calcd for $\mathrm{C}_{24} \mathrm{H}_{28} \mathrm{NO}_{3}{ }^{+} \mathrm{m} / z$ 378.2064, found $\mathrm{m} / z 378.2065$

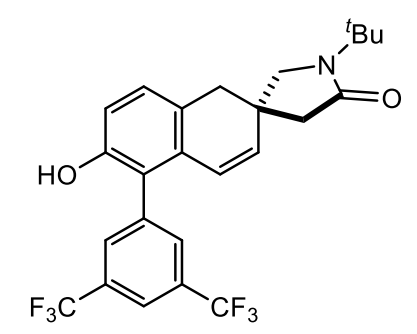

5-(3,5-Bis(trifluoromethyl)phenyl)-1'-(tert-butyl)-6-hydroxy-1Hspiro[naphthalene-2,3'-pyrrolidin]-5'-one (4e)

Prepared according to the general procedure A (Method B) using 1e (30.0 mg), and isolated as colorless oil (13.4 mg, 47\% yield, $0.059 \mathrm{mmol} \mathrm{scale)}$ ); $\mathrm{R}_{f}=0.5$ ( $n$-hexane/EtOAc, $1 / 1)$; ${ }^{1} \mathrm{H} \mathrm{NMR}\left(600 \mathrm{MHz} \mathrm{CDCl}_{3}\right) \delta 7.88(\mathrm{~s}, 1 \mathrm{H}), 7.77(\mathrm{~s}, 1 \mathrm{H})$, $7.74(\mathrm{~s}, 1 \mathrm{H}), 7.05(\mathrm{~d}, J=8.4 \mathrm{~Hz}, 1 \mathrm{H}), 6.78(\mathrm{~d}, J=8.4 \mathrm{~Hz}, 1 \mathrm{H}), 6.32(\mathrm{br} \mathrm{s}, 1 \mathrm{H})$, $6.08(\mathrm{~d}, J=9.6 \mathrm{~Hz}, 1 \mathrm{H}), 5.84(\mathrm{~d}, J=9.6 \mathrm{~Hz}, 1 \mathrm{H}), 3.41(\mathrm{~d}, J=10.2 \mathrm{~Hz}, 1 \mathrm{H}), 3.35$ $(\mathrm{d}, J=10.2 \mathrm{~Hz}, 1 \mathrm{H}), 2.88(\mathrm{~d}, J=15.0 \mathrm{~Hz}, 1 \mathrm{H}), 2.82(\mathrm{~d}, J=15.0 \mathrm{~Hz}, 1 \mathrm{H}), 2.38$ 
(d, $J=17.4 \mathrm{~Hz}, 1 \mathrm{H}), 2.35(\mathrm{~d}, J=17.4 \mathrm{~Hz}, 1 \mathrm{H}), 1.40(\mathrm{~s}, 9 \mathrm{H}) ;{ }^{13} \mathrm{C} \mathrm{NMR}(150 \mathrm{MHz}$, $\left.\mathrm{CDCl}_{3}\right) \delta 174.1,152.4,137.9,136.0,131.9,131.8$ ( $\left.J=27.3 \mathrm{~Hz}, 2 \mathrm{C}\right), 131.3,129.8$, $125.5,125.3,123.5(J=270.0 \mathrm{~Hz}, 2 \mathrm{C}), 123.2,121.5(J=3.0 \mathrm{~Hz}, 2 \mathrm{C}), 114.5$, 55.9, 54.4, 44.7, 39.3, 36.2, 27.9 (3C); ${ }^{19} \mathrm{~F} \mathrm{NMR} \mathrm{(564} \mathrm{MHz} \mathrm{CDCl}_{3}$ ) $\delta$-62.6; IR (ATR) 3331, 1659, 1577, 1456, 1424, 1369, 1279, 1218, $1176 \mathrm{~cm}^{-1}$; HRMS (ESITOF) $[\mathrm{M}+\mathrm{Na}]^{+}$calcd for $\mathrm{C}_{25} \mathrm{H}_{23} \mathrm{~F}_{6} \mathrm{NNaO}_{2}{ }^{+} \mathrm{m} / z 506.1525$, found $\mathrm{m} / z 506.1536$

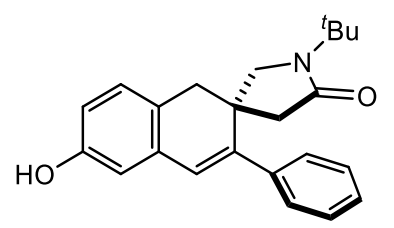

1'-(tert-Butyl)-6-hydroxy-3-phenyl-1H-spiro[naphthalene-2,3'-pyrrolidin]-5'one (4f)

Prepared according to the general procedure A (Method B) using $\mathbf{1 f}$ (37.3 $\mathrm{mg}$ ), $\mathrm{CH}_{2} \mathrm{Cl}_{2}(0.01 \mathrm{M})$, and MS $3 \AA$ (100 mg) and isolated as white solid (22.5 mg, $65 \%$ yield).: m.p. $230-232{ }^{\circ} \mathrm{C} ; \mathrm{R}_{f}=0.3$ ( $n$-hexane/EtOAc, $\left.1 / 1\right) ;{ }^{1} \mathrm{H}$ NMR $(600$ $\left.\mathrm{MHz} \mathrm{CDCl}_{3}\right) \delta 7.37(\mathrm{dd}, J=8.4,7.2 \mathrm{~Hz}, 2 \mathrm{H}), 7.32(\mathrm{t}, J=7.2 \mathrm{~Hz}, 1 \mathrm{H}), 7.22(\mathrm{~d}$, $J=8.4 \mathrm{~Hz}, 2 \mathrm{H}), 7.03(\mathrm{~d}, J=8.4 \mathrm{~Hz}, 1 \mathrm{H}), 6.66(\mathrm{~d}, J=8.4 \mathrm{~Hz}, 1 \mathrm{H}), 6.62(\mathrm{~s}, 1 \mathrm{H})$, $6.42(\mathrm{~s}, 1 \mathrm{H}), 5.00(\mathrm{br} \mathrm{s}, 1 \mathrm{H}), 3.51(\mathrm{~d}, J=9.0 \mathrm{~Hz}, 1 \mathrm{H}), 3.38(\mathrm{~d}, J=9.0 \mathrm{~Hz}, 1 \mathrm{H})$, $2.97(\mathrm{~d}, J=15.0 \mathrm{~Hz}, 1 \mathrm{H}), 2.90(\mathrm{~d}, J=15.0 \mathrm{~Hz}, 1 \mathrm{H}), 2.74(\mathrm{~d}, J=15.6 \mathrm{~Hz}, 1 \mathrm{H})$, $2.32(\mathrm{~d}, J=15.6 \mathrm{~Hz}, 1 \mathrm{H}), 1.32(\mathrm{~s}, 9 \mathrm{H}) ;{ }^{13} \mathrm{C}$ NMR (150 MHz, DMSO-d6) $\delta 172.4$, $156.4,145.4,140.1,134.3,128.9,128.4$ (2C), 127.9, 127.8 (2C), 127.5, 123.2, 114.3, 113.5, 53.3, 53.0, 43.8, 40.6, 38.1, 27.2 (3C); IR (ATR) 2956, 2829, 1749, 1648, 1601, 1514, 1444, 1430, 1364, 1281, 1243, $1218 \mathrm{~cm}^{-1}$; HRMS (ESI-TOF) $[\mathrm{M}+\mathrm{Na}]^{+}$calcd for $\mathrm{C}_{23} \mathrm{H}_{25} \mathrm{NNaO}_{2}{ }^{+} \mathrm{m} / z 370.1778$, found $\mathrm{m} / z 370.1762$<smiles>O=C1C[C@@]2(C=Cc3c(ccc(O)c3Br)C2)CN1C(=O)c1ccccc1</smiles>

\section{5-Bromo-1'-(tert-butyl)-6-hydroxy-1H-spiro[naphthalene-2,3'-pyrrolidin]-5'- one (4g)}

Prepared according to the general procedure A (Method B) using 1g (37.6 mg) 
and MS $3 \AA$ (100 mg) and isolated as white solid (16.7 mg, 48\% yield).: m.p. 205-207 ${ }^{\circ} \mathrm{C} ; \mathrm{R}_{f}=0.5$ ( $n$-hexane/EtOAc, $\left.1 / 1\right) ;{ }^{1} \mathrm{H}$ NMR (600 MHz, $\left.\mathrm{CD}_{3} \mathrm{OD}\right) \delta$ $6.96(\mathrm{~d}, J=8.4 \mathrm{~Hz}, 1 \mathrm{H}), 6.91(\mathrm{~d}, J=9.6 \mathrm{~Hz}, 1 \mathrm{H}), 6.73(\mathrm{~d}, J=8.4 \mathrm{~Hz}, 1 \mathrm{H}), 6.06$ $(\mathrm{d}, J=9.6 \mathrm{~Hz}, 1 \mathrm{H}), 3.45(\mathrm{~d}, J=10.2 \mathrm{~Hz}, 1 \mathrm{H}), 3.41(\mathrm{~d}, J=10.2 \mathrm{~Hz}, 1 \mathrm{H}), 2.85(\mathrm{~d}$, $J=16.2 \mathrm{~Hz}, 1 \mathrm{H}), 2.78(\mathrm{~d}, J=16.2 \mathrm{~Hz}, 1 \mathrm{H}), 2.37(\mathrm{~d}, J=16.2 \mathrm{~Hz}, 1 \mathrm{H}), 2.31(\mathrm{~d}$, $J=16.2 \mathrm{~Hz}, 1 \mathrm{H}), 1.39$ (s, 9H); ${ }^{13} \mathrm{C} \mathrm{NMR}\left(150 \mathrm{MHz}, \mathrm{CD}_{3} \mathrm{OD}\right) \delta 176.0,154.5$, 137.4, 134.1, 129.0, 128.2, 127.0, 115.5, 111.1, 56.4, 55.4, 45.9, 39.7, 37.4, 27.9 (3C); IR (ATR) 3373, 2973, 2925, 2485, 2077, 1651, 1471, 1418, 1295, 1217, $1117 \mathrm{~cm}^{-1}$; HRMS (ESI-TOF) $[\mathrm{M}+\mathrm{Na}]+$ calcd for $\mathrm{C}_{17} \mathrm{H}_{20} \mathrm{BrNNaO}_{2}{ }^{+} \mathrm{m} / Z$ 372.0570 , found $\mathrm{m} / \mathrm{z} 372.0572$

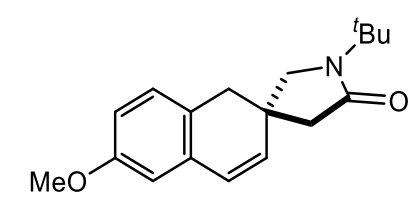

1'-(tert-Butyl)-6-methoxy-1H-spiro[naphthalene-2,3'-pyrrolidin]-5'-one (4h)

Prepared according to the general procedure A (Method B) using $1 \mathrm{~h}$ (31.1 mg) and MS $3 \AA$ (100 mg), and isolated as colorless oil (21.6 mg, 76\% yield).; $\mathrm{R}_{f}=$ 0.6 ( $n$-hexane/EtOAc, $1 / 1) ;{ }^{1} \mathrm{H}$ NMR (600 $\left.\mathrm{MHz} \mathrm{CDCl}_{3}\right) \delta 7.03(\mathrm{~d}, J=8.4 \mathrm{~Hz}$, $1 \mathrm{H}), 6.65(\mathrm{~d}, J=2.4 \mathrm{~Hz}, 1 \mathrm{H}), 6.44(\mathrm{~d}, J=9.6 \mathrm{~Hz}, 1 \mathrm{H}), 5.88(\mathrm{~d}, J=9.6 \mathrm{~Hz}, 1 \mathrm{H})$, $5.71(\mathrm{dd}, J=8.4,2.4 \mathrm{~Hz}, 1 \mathrm{H}), 3.80(\mathrm{~s}, 3 \mathrm{H}), 3.37(\mathrm{~d}, J=8.4 \mathrm{~Hz}, 1 \mathrm{H}), 3.27(\mathrm{~d}, J$ $=8.4 \mathrm{~Hz}, 1 \mathrm{H}), 2.83(\mathrm{~d}, J=16.2 \mathrm{~Hz}, 1 \mathrm{H}), 2.81(\mathrm{~d}, J=16.2 \mathrm{~Hz}, 1 \mathrm{H}), 2.41(\mathrm{~d}, J=$ $16.8 \mathrm{~Hz}, 1 \mathrm{H}), 2.35(\mathrm{~d}, J=16.8 \mathrm{~Hz}, 1 \mathrm{H}), 1.38(\mathrm{~s}, 9 \mathrm{H}) ;{ }^{13} \mathrm{C} \mathrm{NMR}(150 \mathrm{MHz}$, $\left.\mathrm{CDCl}_{3}\right) \delta 173.9,158.9,135.1,134.1,129.2,128.5,125.2,112.6,112.2,55.5,55.5$, 54.1, 45.8, 38.6, 36.7, 27.9 (3C); IR (ATR) 3352, 2971, 2871, 1682, 1604, 1573, 1494, 1404, 1364, 1300, 1261, $1222 \mathrm{~cm}^{-1}$; HRMS (ESI-TOF) $[\mathrm{M}+\mathrm{Na}]^{+}$calcd for $\mathrm{C}_{18} \mathrm{H}_{23} \mathrm{NNaO}_{2}{ }^{+} \mathrm{m} / \mathrm{z} 308.1621$, found $\mathrm{m} / \mathrm{z} 308.1635$

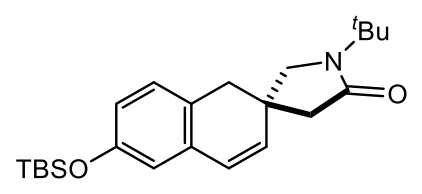

1'-(tert-Butyl)-6-((tert-butyldimethylsilyl)oxy)-1H-spiro[naphthalene-2,3'pyrrolidin]-5'-one (4i)

Prepared according to the general procedure A using $1 \mathrm{i}$ (41.2 mg) and MS $3 \AA$ 
(100 mg), and isolated as colorless oil (37.2 mg, 96\% yield).; $\mathrm{R}_{f}=0.8\left(n^{-}\right.$ hexane/EtOAc, 1/1); ${ }^{1} \mathrm{H} \mathrm{NMR}\left(600 \mathrm{MHz} \mathrm{CDCl}_{3}\right) \delta 6.95(\mathrm{~d}, J=8.4 \mathrm{~Hz}, 1 \mathrm{H})$, $6.63(\mathrm{dd}, J=8.4,3.0 \mathrm{~Hz}, 1 \mathrm{H}), 6.56(\mathrm{~d}, J=3.0 \mathrm{~Hz}, 1 \mathrm{H}), 6.39(\mathrm{~d}, J=9.6 \mathrm{~Hz}, 1 \mathrm{H})$, $5.84(\mathrm{~d}, J=9.6 \mathrm{~Hz}, 1 \mathrm{H}), 3.36(\mathrm{~d}, J=9.6 \mathrm{~Hz}, 1 \mathrm{H}), 3.27(\mathrm{~d}, J=9.6 \mathrm{~Hz}, 1 \mathrm{H}), 2.81$ $(\mathrm{d}, J=15.6 \mathrm{~Hz}, 1 \mathrm{H}), 2.78(\mathrm{~d}, J=15.6 \mathrm{~Hz}, 1 \mathrm{H}), 2.39(\mathrm{~d}, J=16.2 \mathrm{~Hz}, 1 \mathrm{H}), 2.34$ $(\mathrm{d}, J=16.2 \mathrm{~Hz}, 1 \mathrm{H}), 1.38(\mathrm{~s}, 9 \mathrm{H}), 0.98(\mathrm{~s}, 9 \mathrm{H}), 0.20(\mathrm{~s}, 6 \mathrm{H}) ;{ }^{13} \mathrm{C} \mathrm{NMR}(150 \mathrm{MHz}$, $\left.\mathrm{CDCl}_{3}\right) \delta 173.9,154.8,134.7,134.1,129.2,128.5,125.8,118.8,118.1,55.6,54.1$,

45.8, 38.7, 36.7, 27.9 (3C), 25.8 (3C), 18.3, -4.3 (2C); IR (ATR) 2957, 2929, 2858, 1747, 1688, 1604, 1568, 1492, 1401, 1363, $1277 \mathrm{~cm}^{-1}$; HRMS (ESI-TOF) $[\mathrm{M}+\mathrm{Na}]+$ calcd for $\mathrm{C}_{23} \mathrm{H}_{35} \mathrm{NNaO}_{2} \mathrm{Si}^{+} \mathrm{m} / z$ 408.2329, found $\mathrm{m} / z 408.2343$

\section{Characterization of $5 a$ and $6 a$}<smiles>CC12C=C[C@@H]3c4ccc(O)cc4C=CC3C1C(=O)C2</smiles>

\section{2-(tert-Butyl)-7-hydroxy-2,3,9b,9c-tetrahydro- $1 H$ -}

\section{naphtho[2',1':1,3]cyclopropa[1,2-c]pyrrol-1-one (5a)}

Prepared according to general procedure $\mathrm{A}$ using $\mathrm{Rh}_{2}(\mathrm{OAc})_{4}(4.4 \mathrm{mg})$ instead of $\mathrm{AgNTf}_{2}$, and isolated yellow solid (23.7 $\mathrm{mg}$, 88\% yield) (preparative thin layer chromatography condition: toluene/EtOAc 2/1).: m.p. $206-208{ }^{\circ} \mathrm{C} ; \mathrm{R}_{f}=$ 0.4 ( $n$-hexane/EtOAc, $1 / 1) ;{ }^{1} \mathrm{H} \mathrm{NMR}\left(600 \mathrm{MHz} \mathrm{CDCl}_{3}\right) \delta 7.25(\mathrm{~d}, J=8.4 \mathrm{~Hz}$, $1 \mathrm{H}), 6.77(\mathrm{dd}, J=8.4,2.4 \mathrm{~Hz}, 1 \mathrm{H}), 6.62(\mathrm{~d}, J=2.4 \mathrm{~Hz}, 1 \mathrm{H}), 6.29(\mathrm{~d}, J=9.6 \mathrm{~Hz}$, 1H), 6.08 (br s, 1H), 6.08 (d, $J=9.6 \mathrm{~Hz}, 1 \mathrm{H}), 3.76(\mathrm{~d}, J=10.8 \mathrm{~Hz}, 1 \mathrm{H}), 3.59$ (d, $J=10.8 \mathrm{~Hz}, 1 \mathrm{H}), 2.56(\mathrm{~d}, J=1.8 \mathrm{~Hz}, 1 \mathrm{H}), 1.41(\mathrm{~s}, 9 \mathrm{H}), 0.72(\mathrm{~d}, J=1.8 \mathrm{~Hz}, 1 \mathrm{H})$; ${ }^{13} \mathrm{C} \mathrm{NMR}\left(150 \mathrm{MHz} \mathrm{CDCl}_{3}\right.$ ) $\delta 175.7,154.9,132.4,130.0,126.1,125.4,124.1$, 115.5, 114.7, 54.0, 52.1, 33.4, 28.0, 27.8 (3C), 26.3; IR (ATR) 3250, 2967, 2927, 1708, 1636, 1562, 1486, 1417, 1365, 1277, $1216 \mathrm{~cm}^{-1}$; HRMS (ESI-TOF) [M + $\mathrm{Na}]^{+}$calcd for $\mathrm{C}_{17} \mathrm{H}_{19} \mathrm{NNaO}_{2}{ }^{+} \mathrm{m} / z$ 292.1308, found $\mathrm{m} / z$ 292.1310. 
<smiles>O=C1C2=Cc3ccc(O)cc3C=CC2CN1C(=O)c1ccccc1</smiles>

2-(tert-Butyl)-7-hydroxy-1,10a-dihydrobenzo[4,5]cyclohepta[1,2-c]pyrrol$3(2 H)$-one (6a)

Prepared according to general procedure A using $\mathrm{Rh}_{2}(\mathrm{OAc})_{4}(4.4 \mathrm{mg})$ instead of $\mathrm{AgNTf}_{2}$, and isolated yellow solid (2.4 mg, 9\% yield) (preparative thin layer chromatography condition: toluene/EtOAc 2/1): m.p. $216-218^{\circ} \mathrm{C} ; \mathrm{R}_{f}=0.4\left(n^{-}\right.$ hexane/EtOAc, 1/1); ${ }^{1} \mathrm{H} \mathrm{NMR}\left(400 \mathrm{MHz}, \mathrm{CDCl}_{3}\right) \delta 7.72(\mathrm{~d}, J=7.6 \mathrm{~Hz}, 1 \mathrm{H})$, $7.71(\mathrm{br} \mathrm{s}, 1 \mathrm{H}), 7.70(\mathrm{~d}, J=7.6 \mathrm{~Hz}, 1 \mathrm{H}), 7.48(\mathrm{~d}, J=8.8 \mathrm{~Hz}, 1 \mathrm{H}), 7.16(\mathrm{~s}, 1 \mathrm{H})$, $7.13(\mathrm{~d}, J=8.8 \mathrm{~Hz}, 1 \mathrm{H}), 5.21(\mathrm{~s}, 1 \mathrm{H}), 4.70(\mathrm{dd}, J=5.2,2.4 \mathrm{~Hz}, 1 \mathrm{H}), 3.28(\mathrm{dd}, J$ $=14.8,5.2 \mathrm{~Hz}, 1 \mathrm{H}), 2.74(\mathrm{dd}, J=14.8,2.4 \mathrm{~Hz}, 1 \mathrm{H}), 1.26(\mathrm{~s}, 9 \mathrm{H}) ;{ }^{13} \mathrm{C} \mathrm{NMR}(150$ $\left.\mathrm{MHz} \mathrm{CDCl}_{3}\right) \delta 167.7,154.0,136.2,134.6,129.8,128.8,127.5,126.0,124.3$, 118.5, 109.7, 54.9, 53.5, 45.9, 28.3 (3C); IR (ATR) 3287, 2973, 2929, 1711, 1635, 1607, 1446, 1366, 1287, $1226 \mathrm{~cm}^{-1}$; HRMS (ESI-TOF) $[\mathrm{M}+\mathrm{Na}]^{+}$calcd for $\mathrm{C}_{17} \mathrm{H}_{19} \mathrm{NNaO}_{2}{ }^{+} \mathrm{m} / z$ 292.1308, found $\mathrm{m} / z 292.1315$.

\section{Procedure for Dearomatizative Spirocyclization with Water and Oxidation}

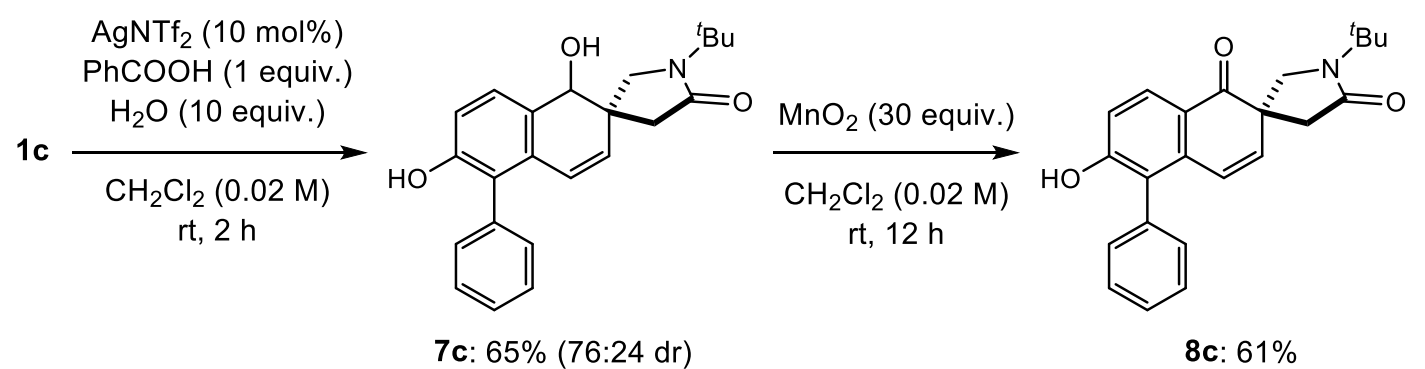

A pre-dried $30 \mathrm{~mL}$ eggplant-shaped flask equipped with a magnetic stir bar was charged with $\operatorname{AgNTf}_{2}$ (5.8 mg, $0.015 \mathrm{mmol}, 10 \mathrm{~mol} \%$ ), benzoic acid (18.3 $\mathrm{mg}, 0.15 \mathrm{mmol}, 1$ equiv.), and $\mathrm{H}_{2} \mathrm{O}$ ( $27 \mu \mathrm{L}, 1.5 \mathrm{mmol}, 10$ equiv.), which were subsequently dissolved partially in $\mathrm{CH}_{2} \mathrm{Cl}_{2}(1.5 \mathrm{~mL})$ under an argon gas atmosphere. Diazo compound $1 \mathrm{c}(56.0 \mathrm{mg}, 0.15 \mathrm{mmol})$ in $\mathrm{CH}_{2} \mathrm{Cl}_{2}(6 \mathrm{~mL})$ was added slowly over $0.5 \mathrm{~h}$ via a syringe pump to the suspension at room temperature, and the reaction mixture was stirred for $0.5 \mathrm{~h}$ at the same temperature. The reaction was quenched with the addition of water. The 
aqueous solution was extracted with $\mathrm{CH}_{2} \mathrm{Cl}_{2} \times 2$. The combined organic layers were washed with brine, dried over $\mathrm{Na}_{2} \mathrm{SO}_{4}$, concentrated under reduced pressure. The obtained crude residue was purified by flash column chromatography (column condition; gradient elution: $n$-hexane/EtOAc, $1 / 1 \rightarrow$ $1 / 3$ ) to afford spirocyclic compound $7 \mathrm{c}$ as colorless oil (35.5 mg, 65\% yield, $76: 24 \mathrm{dr})$.

A pre-dried $30 \mathrm{~mL}$ eggplant-shaped flask were equipped with a magnetic stir bar was charged with 7c (35.3 $\mathrm{mg}, 0.098 \mathrm{mmol})$, which was subsequently dissolved in $\mathrm{CH}_{2} \mathrm{Cl}_{2}(4.9 \mathrm{~mL})$ under an argon gas atmosphere. To a stirred solution was added $\mathrm{MnO}_{2}(252.1 \mathrm{mg}, 2.9 \mathrm{mmol}, 30$ equiv.), and the reaction mixture was stirred for $12 \mathrm{~h}$ at room temperature. The reaction was diluted with $\mathrm{CH}_{2} \mathrm{Cl}_{2}$, filtrated through Celite ${ }^{\circledR}$. The filtrate was concentrated under reduced pressure, and purified by flash column chromatography ( $n^{-}$ hexane/EtOAc, 1/2) to afford spirocyclic compound $8 \mathbf{c}$ as pale yellow solid (21.8 mg, 61\% yield).

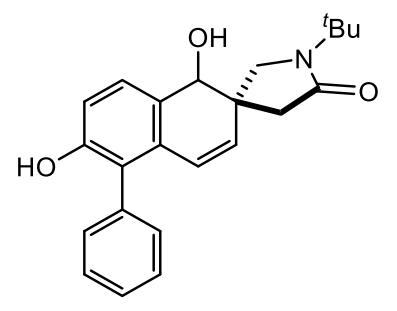

1'-(tert-Butyl)-1,6-dihydroxy-5-phenyl-1H-spiro[naphthalene-2,3'pyrrolidin]-5'-one (7c)

$\mathrm{R}_{f}=0.2$ ( $n$-hexane/EtOAc, $\left.1 / 2\right) ;{ }^{1} \mathrm{H} \mathrm{NMR}\left(600 \mathrm{MHz}, \mathrm{CD}_{3} \mathrm{OD}\right) \delta 7.40(\mathrm{br} \mathrm{s}, 1 \mathrm{H})$, $7.40(\mathrm{dd}, J=7.8,7.8 \mathrm{~Hz}, 2 \mathrm{H}), 7.33(\mathrm{~d}, J=7.8 \mathrm{~Hz}, 1 \mathrm{H}), 7.26(\mathrm{t}, J=7.8 \mathrm{~Hz}, 1 \mathrm{H})$, $7.22(\mathrm{~d}, J=9.6 \mathrm{~Hz}, 1 \mathrm{H}), 7.26-7.19(\mathrm{~m}, 1 \mathrm{H}), 7.16(\mathrm{~d}, J=9.6 \mathrm{~Hz}, 1 \mathrm{H}), 6.80(\mathrm{~d}, J$ $=7.8 \mathrm{~Hz}, 1 \mathrm{H}), 6.19(\mathrm{~d}, J=9.6 \mathrm{~Hz}, 1 \mathrm{H}), 5.81(\mathrm{~d}, J=9.6 \mathrm{~Hz}, 1 \mathrm{H}), 4.44(\mathrm{~s}, 1 \mathrm{H})$, $3.97(\mathrm{~d}, J=10.2 \mathrm{~Hz}, 1 \mathrm{H}), 3.39$ (d, $J=10.2 \mathrm{~Hz}, 1 \mathrm{H}), 2.51$ (d, $J=16.2 \mathrm{~Hz}, 1 \mathrm{H})$, $2.12(\mathrm{~d}, J=16.2 \mathrm{~Hz}, 1 \mathrm{H}), 1.41(\mathrm{~s}, 9 \mathrm{H}) ;{ }^{13} \mathrm{C} \mathrm{NMR}\left(150 \mathrm{MHz}, \mathrm{CD}_{3} \mathrm{OD}\right) \delta 176.1$, 155.9, 137.5, 134.8, 132.6, 132.0, 131.9, 131.8, 129.4, 129.3, 129.0, 128.1, 128.0, 126.6, 115.2, 74.7, 55.5, 53.1, 44.1, 42.3, 27.9 (3C) (NMR data of major diastereomer of 7c were shown.); IR (ATR) 2970, 2950, 1746, 1714, 1666, 1646, 
1369, 1316, $1284 \mathrm{~cm}^{-1}$; HRMS (ESI-TOF) $[\mathrm{M}+\mathrm{Na}]^{+}$calcd for $\mathrm{C}_{23} \mathrm{H}_{25} \mathrm{NNaO}_{3}{ }^{+}$ $\mathrm{m} / \mathrm{z} 386.1727$, found $\mathrm{m} / \mathrm{z} 386.1727$.<smiles></smiles>

1'-(tert-Butyl)-6-hydroxy-5-phenyl-1H-spiro[naphthalene-2,3'-pyrrolidine]-

\section{$1,5^{\prime}$-dione (8c)}

m.p. $235-237{ }^{\circ} \mathrm{C} ; \mathrm{R}_{f}=0.4$ ( $n$-hexane/EtOAc, $\left.1 / 2\right) ;{ }^{1} \mathrm{H} \mathrm{NMR} \mathrm{(600} \mathrm{MHz,} \mathrm{CDCl}_{3}$ ) $\delta 8.07(\mathrm{~d}, J=7.8 \mathrm{~Hz}, 1 \mathrm{H}), 7.55(\mathrm{~d}, J=6.0 \mathrm{~Hz}, 1 \mathrm{H}), 7.53(\mathrm{~d}, J=6.0 \mathrm{~Hz}, 1 \mathrm{H})$, $7.48(\mathrm{t}, J=7.2 \mathrm{~Hz}, 1 \mathrm{H}), 7.31(\mathrm{~d}, J=7.2 \mathrm{~Hz}, 1 \mathrm{H}), 7.30(\mathrm{~d}, J=7.2 \mathrm{~Hz}, 1 \mathrm{H}), 7.03$ (d, $J=7.8 \mathrm{~Hz}, 1 \mathrm{H}), 6.69(\mathrm{br} \mathrm{s}, 1 \mathrm{H}), 6.32(\mathrm{~d}, J=10.2 \mathrm{~Hz}, 1 \mathrm{H}), 6.18(\mathrm{~d}, J=10.2$ $\mathrm{Hz}, 1 \mathrm{H}), 3.86(\mathrm{~d}, J=9.6 \mathrm{~Hz}, 1 \mathrm{H}), 3.33(\mathrm{~d}, J=9.6 \mathrm{~Hz}, 1 \mathrm{H}), 2.94(\mathrm{~d}, J=16.2 \mathrm{~Hz}$, $1 \mathrm{H}), 2.30(\mathrm{~d}, J=16.2 \mathrm{~Hz}, 1 \mathrm{H}), 1.42(\mathrm{~s}, 9 \mathrm{H}) ;{ }^{13} \mathrm{C} \mathrm{NMR}\left(150 \mathrm{MHz}, \mathrm{CDCl}_{3}\right) \delta 198.7$, 172.4, 159.6, 137.6, 136.8, 133.2, 131.0, 130.8, 129.6, 129.5 (2C), 128.9, 125.8, 122.4, 122.2, 115.7, 55.3, 54.7, 47.0, 44.4, 27.8 (3C); IR (ATR) 2971, 2751, 2702, 2665, 1745, 1656, 1565, 1434, 1409, 1304, 1262, 1207, $1088 \mathrm{~cm}^{-1}$; HRMS (ESITOF) $[\mathrm{M}+\mathrm{Na}]^{+}$calcd for $\mathrm{C}_{23} \mathrm{H}_{23} \mathrm{NNaO}_{3}{ }^{+} \mathrm{m} / z$ 384.1570, found $\mathrm{m} / z 384.1572$.

General Procedure B for One-pot Double Dearomative Spirocyclization
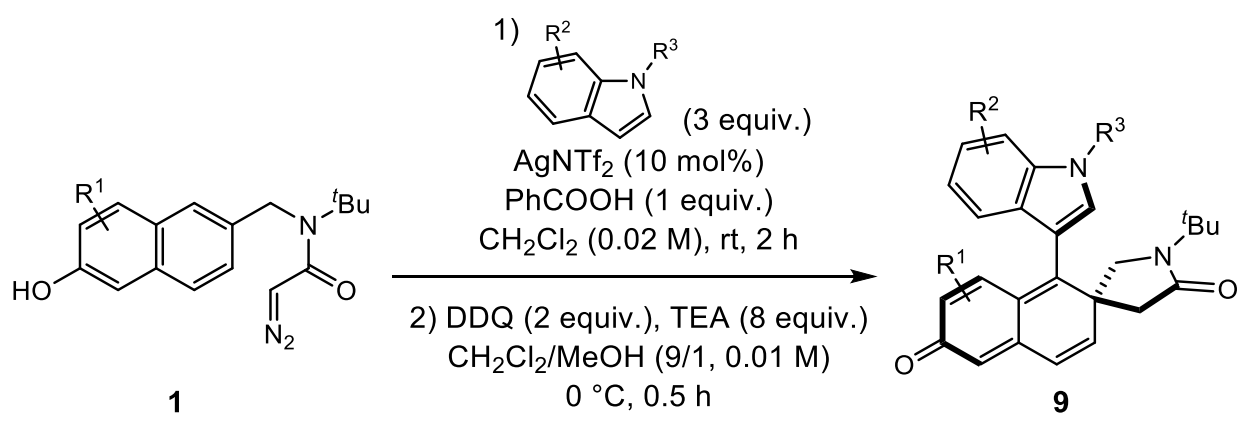
A pre-dried $30 \mathrm{~mL}$ eggplant-shaped flask equipped with a magnetic stir bar was charged with $\mathrm{AgNTf}_{2}$ (3.9 mg, $0.01 \mathrm{mmol}, 10 \mathrm{~mol} \%$ ), an indole derivative (0.3 mmol, 3 equiv.), benzoic acid (12.2 mg, $0.1 \mathrm{mmol}, 1$ equiv.), and 1 (0.1 mmol), which were subsequently dissolved partially in $\mathrm{CH}_{2} \mathrm{Cl}_{2}(5 \mathrm{~mL})$ under an argon gas atmosphere. The reaction mixture was stirred for $2 \mathrm{~h}$ at room temperature, and then cooled to $0^{\circ} \mathrm{C}$ in an ice bath. TEA $(111.5 \mu \mathrm{L}, 0.8 \mathrm{mmol}$, 8 equiv.), $\mathrm{MeOH}$ ( $1 \mathrm{~mL}$ ), and DDQ (45.4 mg, $0.2 \mathrm{mmol}, 2$ equiv.) in $\mathrm{CH}_{2} \mathrm{Cl}_{2}$ (4 $\mathrm{mL}$ ) was added dropwise to the reaction mixture, and stirring was continued for $0.5 \mathrm{~h}$ at the same temperature. The reaction was quenched with the addition of $1 \mathrm{~N}$ aqueous $\mathrm{NaOH}$. The aqueous solution was extracted with $\mathrm{CH}_{2} \mathrm{Cl}_{2} \times 3$, and the combined organic layers were washed with brine, dried over $\mathrm{Na}_{2} \mathrm{SO}_{4}$, concentrated under reduced pressure. The obtained crude residue was purified by flash column chromatography (column condition; diol $\mathrm{SiO}_{2}$, gradient elution: $n$-hexane/EtOAc, $2 / 1 \rightarrow 1 / 4$ ) to afford 9.

NMR data of major diastereomers of $9 \mathbf{a}-\mathbf{i}, \mathbf{k}, \mathbf{l}$ and $10 \mathbf{a}$ were shown.

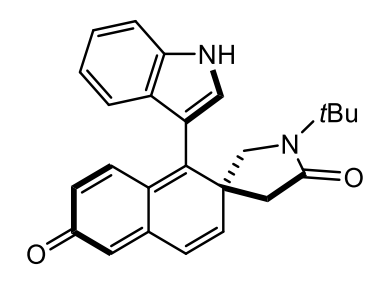

\section{1'-(tert-Butyl)-1-(1H-indol-3-yl)-6H-spiro[naphthalene-2,3'-pyrrolidine]-5',6- dione (9a)}

Prepared according to the general procedure B using $1 \mathrm{a}$ and indole, and isolated as orange solid $(37.4 \mathrm{mg}, 97 \%$ yield $)$. m.p. $159-161{ }^{\circ} \mathrm{C} ; \mathrm{R}_{f}=0.2\left(\mathrm{n}^{-}\right.$ hexane/EtOAc, 1/3); ${ }^{1} \mathrm{H} \mathrm{NMR}\left(600 \mathrm{MHz} \mathrm{CDCl}_{3}\right) \delta 9.32$ (br s, 1H), 7.48 (d, $J=$ $8.4 \mathrm{~Hz}, 1 \mathrm{H}), 7.33(\mathrm{~d}, J=1.8 \mathrm{~Hz}, 1 \mathrm{H}), 7.30(\mathrm{~d}, J=8.4 \mathrm{~Hz}, 1 \mathrm{H}), 7.27(\mathrm{dd}, J=8.4$, $7.2 \mathrm{~Hz}, 1 \mathrm{H}), 7.16(\mathrm{dd}, J=8.4,7.2 \mathrm{~Hz}, 1 \mathrm{H}), 7.01(\mathrm{~d}, J=9.6 \mathrm{~Hz}, 1 \mathrm{H}), 6.66(\mathrm{~d}, J$ $=9.6 \mathrm{~Hz}, 1 \mathrm{H}), 6.35(\mathrm{~d}, J=9.6 \mathrm{~Hz}, 1 \mathrm{H}), 6.29(\mathrm{~d}, J=1.8 \mathrm{~Hz}, 1 \mathrm{H}), 6.24(\mathrm{~d}, J=$ 9.6, $1.8 \mathrm{~Hz}, 1 \mathrm{H}), 3.51(\mathrm{~d}, J=10.8 \mathrm{~Hz}, 1 \mathrm{H}), 3.45(\mathrm{~d}, J=10.8 \mathrm{~Hz}, 1 \mathrm{H}), 2.91(\mathrm{~d}, J$ $=18.0 \mathrm{~Hz}, 1 \mathrm{H}), 2.80(\mathrm{~d}, J=18.0 \mathrm{~Hz}, 1 \mathrm{H}), 0.84(\mathrm{~s}, 9 \mathrm{H}) ;{ }^{13} \mathrm{C} \mathrm{NMR}(150 \mathrm{MHz}$, $\left.\mathrm{CDCl}_{3}\right) \delta 187.9,172.3,153.8,140.2,139.6,137.4,135.6,129.5,128.7,128.1$, $126.1,124.0,123.4,123.3,121.3,118.9$, 111.9, 110.5, 54.8, 54.2, 44.5, 43.1, 
27.0 (3C); IR (ATR) 3134, 2971, 2923, 1748, 1672, 1621, 1549, 1509, 1453, 1410, $1217 \mathrm{~cm}^{-1}$; HRMS (ESI-TOF) $[\mathrm{M}+\mathrm{Na}]^{+}$calcd for $\mathrm{C}_{25} \mathrm{H}_{24} \mathrm{~N}_{2} \mathrm{NaO}_{2}{ }^{+} \mathrm{m} / z$ 407.1730, found $\mathrm{m} / \mathrm{z} 407.1739$.

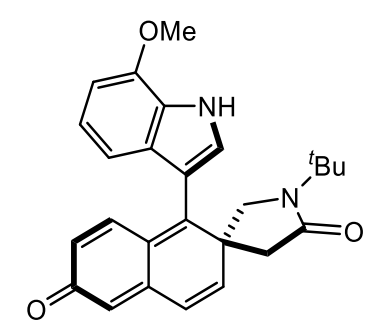

\section{1'-(tert-Butyl)-1-(7-methoxy-1H-indol-3-yl)-6H-spiro[naphthalene-2,3'- pyrrolidine]-5',6-dione (9b)}

Prepared according to the general procedure B using 1a and 7-methoxyindole, and isolated as orange solid (39.6 mg, 95\% yield).: m.p. $155-156{ }^{\circ} \mathrm{C} ; \mathrm{R}_{f}=0.2$ ( $n$-hexane/EtOAc, $1 / 3) ;{ }^{1} \mathrm{H}$ NMR $\left(600 \mathrm{MHz} \mathrm{CDCl}_{3}\right) \delta 8.81$ (br s, 1H), $7.29(\mathrm{~d}$, $J=1.8 \mathrm{~Hz}, 1 \mathrm{H}), 7.08(\mathrm{dd}, J=7.8,7.2 \mathrm{~Hz}, 1 \mathrm{H}), 7.00(\mathrm{~d}, J=10.2 \mathrm{~Hz}, 1 \mathrm{H}), 6.87$ $(\mathrm{d}, J=7.8 \mathrm{~Hz}, 1 \mathrm{H}), 6.71(\mathrm{~d}, J=7.2 \mathrm{~Hz}, 1 \mathrm{H}), 6.64(\mathrm{~d}, J=9.0 \mathrm{~Hz}, 1 \mathrm{H}), 6.35(\mathrm{~d}, J$ $=9.0 \mathrm{~Hz}, 1 \mathrm{H}), 6.28(\mathrm{~d}, J=1.8 \mathrm{~Hz}, 1 \mathrm{H}), 6.23(\mathrm{dd}, J=10.2,1.8 \mathrm{~Hz}, 1 \mathrm{H}), 3.99(\mathrm{~s}$, $3 \mathrm{H}), 3.47(\mathrm{~d}, J=10.2 \mathrm{~Hz}, 1 \mathrm{H}), 3.44(\mathrm{~d}, J=10.2 \mathrm{~Hz}, 1 \mathrm{H}), 2.91(\mathrm{~d}, J=17.4 \mathrm{~Hz}$, $1 \mathrm{H}), 2.73(\mathrm{~d}, J=17.4 \mathrm{~Hz}, 1 \mathrm{H}), 0.88(\mathrm{~s}, 9 \mathrm{H}) ;{ }^{13} \mathrm{C} \mathrm{NMR}\left(150 \mathrm{MHz}, \mathrm{CDCl}_{3}\right) \delta 187.8$, 172.1, 153.5, 146.5, 140.5, 139.6, 137.4, 129.9, 129.4, 128.2, 126.2, 125.9, 124.0, $122.8,121.1,111.5,111.1,102.9,55.7,54.7,54.2,44.5,43.0,27.1$ (3C); IR (ATR) 3204, 2925, 2853, 1746, 1668, 1620, 1544, 1420, 1366, $1217 \mathrm{~cm}^{-1}$; HRMS (ESI-TOF) $[\mathrm{M}+\mathrm{Na}]^{+}$calcd for $\mathrm{C}_{26} \mathrm{H}_{26} \mathrm{~N}_{2} \mathrm{NaO}_{3}{ }^{+} \mathrm{m} / z$ 437.1836, found $m / z 437.1843$.

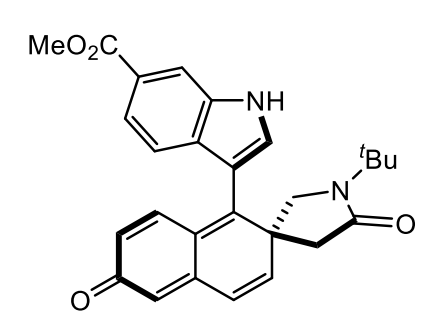

Methyl 3-(1'-(tert-butyl)-5',6-dioxo-6H-spiro[naphthalene-2,3'-pyrrolidin]-1yl)-1 $H$-indole-6-carboxylate (9c)

Prepared according to the general procedure B using 1a and methyl indole-6- 
carboxylate, and isolated as orange solid (36.1 mg, 82\% yield). m.p. 155$156{ }^{\circ} \mathrm{C} ; \mathrm{R}_{f}=0.1(n$-hexane/EtOAc, $1 / 2) ;{ }^{1} \mathrm{H} \mathrm{NMR}\left(600 \mathrm{MHz}, \mathrm{CDCl}_{3}\right) \delta 10.21(\mathrm{br}$ s, $1 \mathrm{H}), 8.28(\mathrm{~d}, J=1.8 \mathrm{~Hz}, 1 \mathrm{H}), 7.86(\mathrm{dd}, J=10.2,1.8 \mathrm{~Hz}, 1 \mathrm{H}), 7.50(\mathrm{~d}, J=3.0$ $\mathrm{Hz}, 1 \mathrm{H}), 7.34(\mathrm{~d}, J=10.2 \mathrm{~Hz}, 1 \mathrm{H}), 6.93(\mathrm{~d}, J=10.2 \mathrm{~Hz}, 1 \mathrm{H}), 6.67(\mathrm{~d}, J=9.6$ $\mathrm{Hz}, 1 \mathrm{H}), 6.35(\mathrm{~d}, J=9.6 \mathrm{~Hz}, 1 \mathrm{H}), 6.30(\mathrm{~d}, J=1.8 \mathrm{~Hz}, 1 \mathrm{H}), 6.24(\mathrm{dd}, J=10.2$, $1.8 \mathrm{~Hz}, 1 \mathrm{H}), 3.97(\mathrm{~s}, 3 \mathrm{H}), 3.55(\mathrm{~d}, J=11.4 \mathrm{~Hz}, 1 \mathrm{H}), 3.43(\mathrm{~d}, J=11.4 \mathrm{~Hz}, 1 \mathrm{H})$, $2.90(\mathrm{~d}, J=17.4 \mathrm{~Hz}, 1 \mathrm{H}), 2.85(\mathrm{~d}, J=17.4 \mathrm{~Hz}, 1 \mathrm{H}), 0.82(\mathrm{~s}, 9 \mathrm{H}) ;{ }^{13} \mathrm{C}$ NMR $(150$ $\left.\mathrm{MHz}_{2} \mathrm{CDCl}_{3}\right) \delta 187.8,172.4,168.0,152.6,140.0,139.5,137.0,135.1,132.0$, $129.7,128.3,126.8,126.3,124.9,124.2,122.2,118.5,114.6,110.6,54.8,54.3$, 52.4, 44.4, 43.2, 27.0 (3C); IR (ATR) 3208, 2972, 2918, 1709, 1661, 1618, 1544, 1434, 1311, 1274, $1214 \mathrm{~cm}^{-1}$; HRMS (ESI-TOF) $[\mathrm{M}+\mathrm{Na}]^{+}$calcd for $\mathrm{C}_{27} \mathrm{H}_{26} \mathrm{~N}_{2} \mathrm{NaO}_{4}{ }^{+} \mathrm{m} / z$ 465.1785, found $\mathrm{m} / z$ 465.1774.

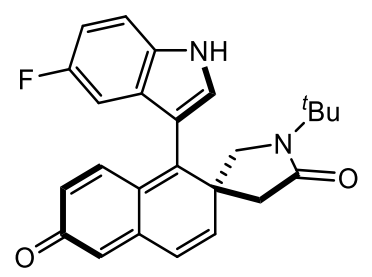

\section{1'-(tert-Butyl)-1-(5-fluoro-1 $H$-indol-3-yl)-6H-spiro[naphthalene-2,3'- pyrrolidine]-5',6-dione (9d)}

Prepared according to the general procedure B using 1a and 5-fluoroindole, and isolated as pale orange solid (34.9 mg, 87\% yield). m.p. $206-208{ }^{\circ} \mathrm{C} ; \mathrm{R}_{f}=$ 0.2 ( $n$-hexane/EtOAc, 1/3); $\left.{ }^{1} \mathrm{H} \mathrm{NMR} \mathrm{(600} \mathrm{MHz,} \mathrm{CDCl}_{3}\right) \delta 9.56$ (br s, $\left.1 \mathrm{H}\right), 7.42$ $(\mathrm{dd}, J=9.0,5.4 \mathrm{~Hz}, 1 \mathrm{H}), 7.37(\mathrm{~d}, J=2.4 \mathrm{~Hz}, 1 \mathrm{H}), 7.02(\mathrm{ddd}, J=9.0,9.0,1.8$ $\mathrm{Hz}, 1 \mathrm{H}), 6.98(\mathrm{~d}, J=10.2 \mathrm{~Hz}, 1 \mathrm{H}), 6.95(\mathrm{dd}, J=9.0,1.8 \mathrm{~Hz}, 1 \mathrm{H}), 6.66(\mathrm{~d}, J=$ $9.6 \mathrm{~Hz}, 1 \mathrm{H}), 6.34(\mathrm{~d}, J=9.6 \mathrm{~Hz}, 1 \mathrm{H}), 6.29(\mathrm{~d}, J=1.8 \mathrm{~Hz}, 1 \mathrm{H}), 6.26(\mathrm{dd}, J=$ $10.2,1.8 \mathrm{~Hz}, 1 \mathrm{H}), 3.53(\mathrm{~d}, J=10.8 \mathrm{~Hz}, 1 \mathrm{H}), 3.41(\mathrm{~d}, J=10.8 \mathrm{~Hz}, 1 \mathrm{H}), 2.89(\mathrm{~d}$, $J=17.4 \mathrm{~Hz}, 1 \mathrm{H}), 2.82(\mathrm{~d}, J=17.4 \mathrm{~Hz}, 1 \mathrm{H}), 0.86(\mathrm{~s}, 9 \mathrm{H}) ;{ }^{13} \mathrm{C} \mathrm{NMR}(150 \mathrm{MHz}$, $\left.\mathrm{CDCl}_{3}\right) \delta 187.8,172.4,158.1,152.9,140.0,139.5,137.1,132.1,129.7,129.0$ $(\mathrm{dd}, J=10.1 \mathrm{~Hz}), 128.1,126.2,125.2,124.2,112.9(\mathrm{~d}, J=10.1 \mathrm{~Hz}), 111.9(\mathrm{~d}, J$ $=25.8 \mathrm{~Hz}), 110.5(\mathrm{~d}, J=4.4 \mathrm{~Hz}), 103.7(\mathrm{~d}, J=24.5 \mathrm{~Hz}), 54.8,54.3,44.4,43.2$, 27.1; ${ }^{19} \mathrm{~F}$ NMR (564 MHz, $\mathrm{CDCl}_{3}$ ) $\delta$-121.9; IR (ATR) 3167, 3111, 2971, 2926, 1739, 1669, 1615, 1542, 1417, $1231 \mathrm{~cm}^{-1}$; HRMS (ESI-TOF) $[\mathrm{M}+\mathrm{Na}]^{+}$calcd for $\mathrm{C}_{25} \mathrm{H}_{23} \mathrm{FN}_{2} \mathrm{NaO}_{2}{ }^{+} \mathrm{m} / z$ 425.1636, found $\mathrm{m} / z 425.1632$. 


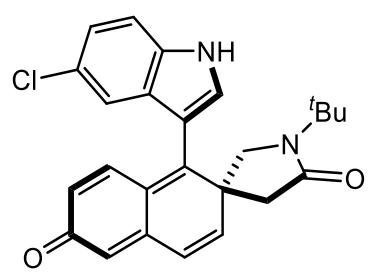

1'-(tert-Butyl)-1-(5-chloro-1H-indol-3-yl)-6H-spiro[naphthalene-2,3'-

\section{pyrrolidine]-5',6-dione (9e)}

Prepared according to the general procedure B using 1a and 5-chloroindole, and isolated as pale orange solid ( $34.6 \mathrm{mg}, 83 \%$ yield). $\mathrm{m} . \mathrm{p} .235-237^{\circ} \mathrm{C} ; \mathrm{R}_{f}=$ 0.1 ( $n$-hexane/EtOAc, 1/3); ${ }^{1} \mathrm{H}$ NMR $\left(600 \mathrm{MHz} \mathrm{CDCl}_{3}\right) \delta 9.79$ (br s, $\left.1 \mathrm{H}\right), 7.42$ $(\mathrm{d}, J=9.0 \mathrm{~Hz}, 1 \mathrm{H}), 7.34(\mathrm{~d}, J=2.4 \mathrm{~Hz}, 1 \mathrm{H}), 7.27(\mathrm{~s}, 1 \mathrm{H}), 7.22(\mathrm{~d}, J=9.0 \mathrm{~Hz}$, $1 \mathrm{H}), 6.95(\mathrm{~d}, J=10.2 \mathrm{~Hz}, 1 \mathrm{H}), 6.67(\mathrm{~d}, J=9.6 \mathrm{~Hz}, 1 \mathrm{H}), 6.34(\mathrm{~d}, J=9.6 \mathrm{~Hz}, 1 \mathrm{H})$, $6.30(\mathrm{~s}, 1 \mathrm{H}), 6.27(\mathrm{~d}, J=10.2 \mathrm{~Hz}, 1 \mathrm{H}), 3.55(\mathrm{~d}, J=10.2 \mathrm{~Hz}, 1 \mathrm{H}), 3.43(\mathrm{~d}, J=$ $10.2 \mathrm{~Hz}, 1 \mathrm{H}), 2.87(\mathrm{~d}, J=17.4 \mathrm{~Hz}, 1 \mathrm{H}), 2.83(\mathrm{~d}, J=17.4 \mathrm{~Hz}, 1 \mathrm{H}), 0.86(\mathrm{~s}, 9 \mathrm{H})$; ${ }^{13} \mathrm{C}$ NMR $\left(150 \mathrm{MHz} \mathrm{CDCl}_{3}\right) \delta 187.8,172.4,152.6,140.0,139.5,137.0,134.0$, 129.8, 129.6, 128.3, 127.1, 126.3, 124.8, 124.2, 123.7, 118.1, 113.2, 110.0, 54.9, 54.3, 44.3, 43.3, 27.0 (3C); IR (ATR) 3104, 2971, 2924, 2877, 1739, 1668, 1612, 1541, 1508, 1416, $1217 \mathrm{~cm}^{-1}$; HRMS (ESI-TOF) $[\mathrm{M}+\mathrm{Na}]+$ calcd for $\mathrm{C}_{25} \mathrm{H}_{23} \mathrm{ClN}_{2} \mathrm{NaO}_{2}{ }^{+} \mathrm{m} / z$ 441.1340, found $\mathrm{m} / z$ 441.1336.

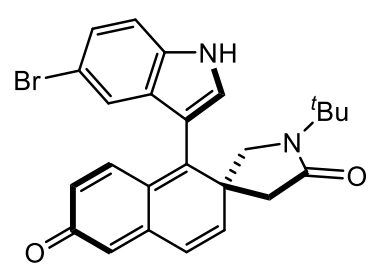

1-(5-Bromo-1H-indol-3-yl)-1'-(tert-butyl)-6H-spiro[naphthalene-2,3'pyrrolidine]-5',6-dione (9f)

Prepared according to the general procedure B using 1a and 5-bromoindole, and isolated as pale orange solid (36.6 mg, $79 \%$ yield).: m.p. $238-240{ }^{\circ} \mathrm{C} ; \mathrm{R}_{f}=$ 0.2 ( $n$-hexane/EtOAc, $1 / 3) ;{ }^{1} \mathrm{H} \mathrm{NMR}\left(600 \mathrm{MHz} \mathrm{CDCl}_{3}\right) \delta 9.54$ (br s, $\left.1 \mathrm{H}\right), 7.43$ $(\mathrm{s}, 1 \mathrm{H}), 7.36(\mathrm{~d}, J=2.4 \mathrm{~Hz}, 1 \mathrm{H}), 7.38-7.35(\mathrm{~m}, 1 \mathrm{H}), 7.32(\mathrm{~d}, J=2.4 \mathrm{~Hz}, 1 \mathrm{H})$, $6.94(\mathrm{~d}, J=10.2 \mathrm{~Hz}, 1 \mathrm{H}), 6.66(\mathrm{~d}, J=9.6 \mathrm{~Hz}, 1 \mathrm{H}), 6.33(\mathrm{~d}, J=9.6 \mathrm{~Hz}, 1 \mathrm{H}), 6.30$ $(\mathrm{d}, J=1.8 \mathrm{~Hz}, 1 \mathrm{H}), 6.27(\mathrm{dd}, J=10.2,1.8 \mathrm{~Hz}, 1 \mathrm{H}), 3.54(\mathrm{~d}, J=10.2 \mathrm{~Hz}, 1 \mathrm{H})$, $3.43(\mathrm{~d}, J=10.2 \mathrm{~Hz}, 1 \mathrm{H}), 2.86(\mathrm{~d}, J=17.4 \mathrm{~Hz}, 1 \mathrm{H}), 2.81(\mathrm{~d}, J=17.4 \mathrm{~Hz}, 1 \mathrm{H})$, 
$0.85(\mathrm{~s}, 9 \mathrm{H}) ;{ }^{13} \mathrm{C} \mathrm{NMR}\left(150 \mathrm{MHz}, \mathrm{CDCl}_{3}\right) \delta$ 187.8, 172.3, 152.4, 140.0, 139.4, 136.9, 134.3, 130.2, 129.9, 128.4, 126.3, 126.3, 124.7, 124.3, 121.2, 114.5, 113.5, 110.0, 54.9, 54.3, 44.3, 43.3, 27.0 (3C); IR (ATR) 3213, 2971, 2924, 1746, 1673, 1610, 1541, 1507, 1413, $1218 \mathrm{~cm}^{-1}$; HRMS (ESI-TOF) $\left[\mathrm{M}+\mathrm{Na}{ }^{+}\right.$calcd for $\mathrm{C}_{25} \mathrm{H}_{23} \mathrm{BrN}_{2} \mathrm{NaO}_{2}{ }^{+} \mathrm{m} / z$ 485.0835, found $\mathrm{m} / z$ 485.0842.

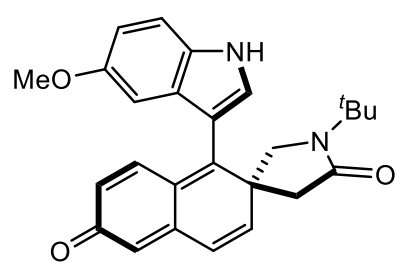

1'-(tert-Butyl)-1-(5-methoxy-1H-indol-3-y])-6H-spiro[naphthalene-2,3'pyrrolidine]-5',6-dione (9g)

Prepared according to the general procedure B using 1a, 5-methoxyindole, and 3 equiv. of DDQ and isolated as orange solid (34.2 mg, 82\% yield).: m.p. $173-175^{\circ} \mathrm{C} ; \mathrm{R}_{f}=0.2$ ( $n$-hexane/EtOAc, $\left.1 / 3\right) ;{ }^{1} \mathrm{H} \mathrm{NMR}\left(600 \mathrm{MHz}, \mathrm{CDCl}_{3}\right) \delta 9.06$ (br s, 1H), $7.36(\mathrm{~d}, J=8.4 \mathrm{~Hz}, 1 \mathrm{H}), 7.28(\mathrm{~s}, 1 \mathrm{H}), 7.04(\mathrm{~d}, J=10.2 \mathrm{~Hz}, 1 \mathrm{H}), 6.93$ $(\mathrm{d}, J=8.4 \mathrm{~Hz}, 1 \mathrm{H}), 6.65(\mathrm{~s}, 1 \mathrm{H}), 6.65(\mathrm{~d}, J=9.6 \mathrm{~Hz}, 1 \mathrm{H}), 6.35(\mathrm{~d}, J=9.6 \mathrm{~Hz}$, $1 \mathrm{H}), 6.29(\mathrm{~s}, 1 \mathrm{H}), 6.26(\mathrm{~d}, J=10.2 \mathrm{~Hz}, 1 \mathrm{H}), 3.76(\mathrm{~s}, 3 \mathrm{H}), 3.50(\mathrm{~d}, J=10.8 \mathrm{~Hz}$, $1 \mathrm{H}), 3.42(\mathrm{~d}, J=10.8 \mathrm{~Hz}, 1 \mathrm{H}), 2.90(\mathrm{~d}, J=16.8 \mathrm{~Hz}, 1 \mathrm{H}), 2.78(\mathrm{~d}, J=16.8 \mathrm{~Hz}$, $1 \mathrm{H}), 0.88(\mathrm{~s}, 9 \mathrm{H}) ;{ }^{13} \mathrm{C} \mathrm{NMR}\left(150 \mathrm{MHz} \mathrm{CDCl}_{3}\right) \delta$ 187.9, 172.3, 157.0, 155.5, 140.3, 139.7, 137.5, 130.7, 129.4, 129.1, 127.8, 126.1, 124.1, 124.0, 113.5, 112.7, 110.3, 100.4, 56.1, 54.9, 54.3, 44.5, 43.1, 27.1 (3C); IR (ATR) 3647, 3469, 3229, 2939, 1739, 1658, 1616, 1544, 1486, 1422, 1288, $1217 \mathrm{~cm}^{-1}$; HRMS (ESI-TOF) $[\mathrm{M}+\mathrm{Na}]^{+}$calcd for $\mathrm{C}_{26} \mathrm{H}_{26} \mathrm{~N}_{2} \mathrm{NaO}_{3}{ }^{+} \mathrm{m} / z$ 437.1836, found $\mathrm{m} / z 437.1849$.

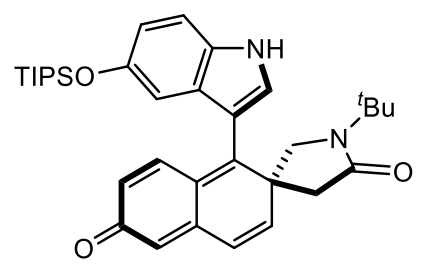

1'-(tert-Butyl)-1-(5-((triisopropylsilyl)oxy)-1H-indol-3-yl)-6Hspiro[naphthalene-2,3'-pyrrolidine]-5',6-dione (9h)

Prepared according to the general procedure $\mathrm{B}$ using $\mathbf{1 a}$ and $5^{-}$ ((triisopropylsilyl)oxy)-indole, and isolated as orange solid (47.1 mg, 83\% 
yield).: m.p. $156-158{ }^{\circ} \mathrm{C} ; \mathrm{R}_{f}=0.3$ ( $n$-hexane/EtOAc, $\left.1 / 2\right) ;{ }^{1} \mathrm{H}$ NMR $(600 \mathrm{MHz}$, $\left.\mathrm{CDCl}_{3}\right) \delta 8.98(\mathrm{br} \mathrm{s}, 1 \mathrm{H}), 7.30(\mathrm{~d}, J=1.2 \mathrm{~Hz}, 1 \mathrm{H}), 7.30(\mathrm{~d}, J=9.0 \mathrm{~Hz}, 1 \mathrm{H}), 7.03$ $(\mathrm{d}, J=9.6 \mathrm{~Hz}, 1 \mathrm{H}), 6.88(\mathrm{dd}, J=9.0,1.8 \mathrm{~Hz}, 1 \mathrm{H}), 6.68(\mathrm{~d}, J=1.8 \mathrm{~Hz}, 1 \mathrm{H}), 6.65$ $(\mathrm{d}, J=9.6 \mathrm{~Hz}, 1 \mathrm{H}), 6.35(\mathrm{~d}, J=9.6 \mathrm{~Hz}, 1 \mathrm{H}), 6.29(\mathrm{~d}, J=1.8 \mathrm{~Hz}, 1 \mathrm{H}), 6.23(\mathrm{dd}$, $J=9.6,1.8 \mathrm{~Hz}, 1 \mathrm{H}), 3.48(\mathrm{~d}, J=10.2 \mathrm{~Hz}, 1 \mathrm{H}), 3.35(\mathrm{~d}, J=10.2 \mathrm{~Hz}, 1 \mathrm{H}), 2.93$ (d, $J=18.0 \mathrm{~Hz}, 1 \mathrm{H}$ ), 2.78 (d, $J=18.0 \mathrm{~Hz}, 1 \mathrm{H}$ ), 1.20 (sep, $J=7.8 \mathrm{~Hz}, 3 \mathrm{H}$ ), 1.05 (d, $J=7.8 \mathrm{~Hz}, 18 \mathrm{H}), 0.91$ (s, 9H); ${ }^{13} \mathrm{C} \mathrm{NMR}\left(150 \mathrm{MHz}, \mathrm{CDCl}_{3}\right) \delta 187.8,172.3$, 153.7, 151.3, 140.2, 139.6, 137.6, 130.7, 129.6, 129.4, 127.7, 126.0, 124.2, 123.9, 117.5, 112.2, 110.2, 107.7, 54.8, 54.3, 44.4, 43.0, 27.1 (3C), 18.1 (6C), 12.7 (3C); IR (ATR) 2942, 2865, 1746, 1672, 1621, 1543, 1469, 1419, 1365, $1217 \mathrm{~cm}^{-1}$; HRMS (ESI-TOF) $[\mathrm{M}+\mathrm{Na}]^{+}$calcd for $\mathrm{C}_{34} \mathrm{H}_{44} \mathrm{~N}_{2} \mathrm{NaO}_{3} \mathrm{Si}^{+} \mathrm{m} / z$ 579.3013, found $\mathrm{m} / \mathrm{z} 579.3002$.

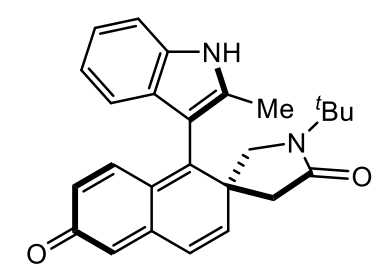

\section{1-(1-Benzyl-1 H-indol-3-yl)-1'-(tert-butyl)-6H-spiro[naphthalene-2,3'- pyrrolidine]-5',6-dione (9i)}

Prepared according to the general procedure B using $1 \mathrm{a}$ and 2-methylindole. Major atropisomer: Orange solid (22.6 mg, $58 \%$ yield): m.p. $174-176{ }^{\circ} \mathrm{C} ; \mathrm{R}_{f}=$ 0.2 ( $n$-hexane/EtOAc, $1 / 3) ;{ }^{1} \mathrm{H} \mathrm{NMR}\left(600 \mathrm{MHz}, \mathrm{CDCl}_{3}\right) \delta 8.44(\mathrm{br} \mathrm{s}, 1 \mathrm{H}), 7.37$ (d, $J=7.8 \mathrm{~Hz}, 1 \mathrm{H}), 7.20(\mathrm{dd}, J=7.8,7.2 \mathrm{~Hz}, 1 \mathrm{H}), 7.15(\mathrm{~d}, J=7.2 \mathrm{~Hz}, 1 \mathrm{H}), 7.10$ (dd, $J=7.2,7.2 \mathrm{~Hz}, 1 \mathrm{H}), 6.82(\mathrm{~d}, J=9.6 \mathrm{~Hz}, 1 \mathrm{H}), 6.67(\mathrm{~d}, J=10.8 \mathrm{~Hz}, 1 \mathrm{H})$, $6.50(\mathrm{~d}, J=9.6 \mathrm{~Hz}, 1 \mathrm{H}), 6.29(\mathrm{~d}, J=2.4 \mathrm{~Hz}, 1 \mathrm{H}), 6.22(\mathrm{dd}, J=10.8,2.4 \mathrm{~Hz}$, $1 \mathrm{H}), 3.87(\mathrm{~d}, J=9.6 \mathrm{~Hz}, 1 \mathrm{H}), 3.42(\mathrm{~d}, J=9.6 \mathrm{~Hz}, 1 \mathrm{H}), 2.83(\mathrm{~d}, J=16.8 \mathrm{~Hz}, 1 \mathrm{H})$, $2.45(\mathrm{~d}, J=16.8 \mathrm{~Hz}, 1 \mathrm{H}), 2.33(\mathrm{~s}, 3 \mathrm{H}), 1.05(\mathrm{~s}, 9 \mathrm{H}) ;{ }^{13} \mathrm{C} \mathrm{NMR}\left(150 \mathrm{MHz}, \mathrm{CDCl}_{3}\right)$ $\delta$ 187.9, 172.0, 153.5, 140.0, 139.7, 136.6, 135.6, 133.5, 131.3, 129.9, 129.5, $125.8,124.0,122.4,120.8,118.5,111.1,108.0,54.3,54.2,47.4,43.4,27.3$ (3C), 13.4; IR (ATR) 3272, 2973, 2925, 1747, 1670, 1621, 1524, 1456, $1228 \mathrm{~cm}^{-1}$; HRMS (ESI-TOF) $[\mathrm{M}+\mathrm{Na}]^{+}$calcd for $\mathrm{C}_{26} \mathrm{H}_{26} \mathrm{~N}_{2} \mathrm{NaO}_{2}{ }^{+} \mathrm{m} / z$ 421.1886, found $\mathrm{m} / \mathrm{z} 421.1893$.

Minor atropisomer: Orange solid (16.7 mg, $42 \%$ yield): m.p. $183-184{ }^{\circ} \mathrm{C} ; \mathrm{R}_{f}=$ 
$0.3(n$-hexane/EtOAc, $1 / 3) ;{ }^{1} \mathrm{H}$ NMR $\left(600 \mathrm{MHz} \mathrm{CDCl}_{3}\right) \delta 8.46$ (br s, $\left.1 \mathrm{H}\right), 7.38$ $(\mathrm{d}, J=7.8 \mathrm{~Hz}, 1 \mathrm{H}), 7.21(\mathrm{dd}, J=7.8,7.8 \mathrm{~Hz}, 1 \mathrm{H}), 7.16(\mathrm{~d}, J=7.8 \mathrm{~Hz}, 1 \mathrm{H}), 7.10$ $(\mathrm{dd}, J=7.8,7.8 \mathrm{~Hz}, 1 \mathrm{H}), 6.78(\mathrm{~d}, J=9.6 \mathrm{~Hz}, 1 \mathrm{H}), 6.67(\mathrm{~d}, J=10.8 \mathrm{~Hz}, 1 \mathrm{H})$, $6.61(\mathrm{~d}, J=10.2 \mathrm{~Hz}, 1 \mathrm{H}), 6.30(\mathrm{~s}, 1 \mathrm{H}), 6.23(\mathrm{~d}, J=9.6 \mathrm{~Hz}, 1 \mathrm{H}), 3.70(\mathrm{~d}, J=9.0$ $\mathrm{Hz}, 1 \mathrm{H}), 3.29(\mathrm{~d}, J=9.0 \mathrm{~Hz}, 1 \mathrm{H}), 3.14(\mathrm{~d}, J=15.6 \mathrm{~Hz}, 1 \mathrm{H}), 2.33(\mathrm{~d}, J=15.6$ $\mathrm{Hz}, 1 \mathrm{H}), 2.26(\mathrm{~s}, 3 \mathrm{H}), 1.31(\mathrm{~s}, 9 \mathrm{H}) ;{ }^{13} \mathrm{C} \mathrm{NMR}\left(150 \mathrm{MHz}, \mathrm{CDCl}_{3}\right) \delta 187.9,171.9$, 153.1, 139.8, 139.6, 136.6, 135.5, 132.9, 132.4, 130.0, 129.4, 125.4, 123.9, 122.7, 121.2, 119.4, 110.9, 108.4, 54.5, 53.7, 48.0, 43.7, 27.8 (3C), 13.0; IR (ATR) 3272, 2972, 2924, 1746, 1671, 1621, 1523, 1456, $1228 \mathrm{~cm}^{-1}$; HRMS (ESI-TOF) [M + $\mathrm{Na}]^{+}$calcd for $\mathrm{C}_{26} \mathrm{H}_{26} \mathrm{~N}_{2} \mathrm{NaO}_{2}{ }^{+} \mathrm{m} / Z$ 421.1886, found $\mathrm{m} / z 421.1882$.

The relative configurations of $\mathbf{9}$ i were determined by NOESY experiments.

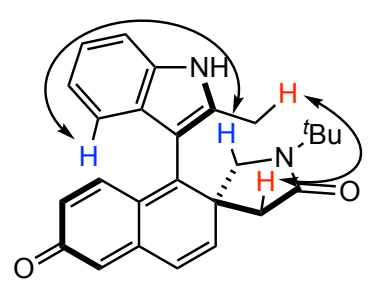

9i (major)

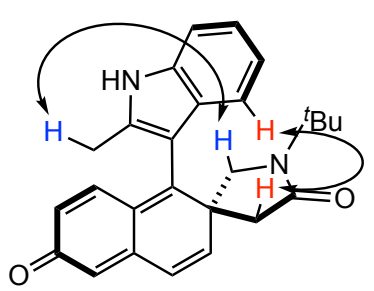

9i (minor)

ح: NOESY correlation

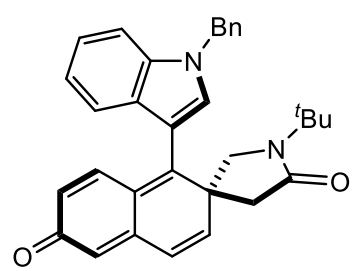

1-(1-Benzyl-1H-indol-3-yl)-1'-(tert-butyl)-6H-spiro[naphthalene-2,3'pyrrolidine]-5',6-dione (9j)

Prepared according to the general procedure B using 1 a and $N$-benzylindole, and isolated as pale orange solid (37.4 mg, 79\% yield). $\mathrm{m} . \mathrm{p} .90-91{ }^{\circ} \mathrm{C} ; \mathrm{R}_{f}=$ 0.2 ( $n$-hexane/EtOAc, $1 / 3) ;{ }^{1} \mathrm{H}$ NMR (600 $\left.\mathrm{MHz} \mathrm{CDCl}_{3}\right) \delta$ 7.38-7.34 (m, 3H), $7.30(\mathrm{dd}, J=7.2,7.2 \mathrm{~Hz}, 2 \mathrm{H}), 7.29(\mathrm{~s}, 1 \mathrm{H}), 7.24(\mathrm{~d}, J=7.2 \mathrm{~Hz}, 2 \mathrm{H}), 7.16$ (d, $J$ $=6.6 \mathrm{~Hz}, 1 \mathrm{H}), 7.16(\mathrm{t}, J=7.2 \mathrm{~Hz}, 1 \mathrm{H}), 7.03(\mathrm{~d}, J=9.6 \mathrm{~Hz}, 1 \mathrm{H}), 6.63(\mathrm{~d}, J=9.6$ $\mathrm{Hz}, 1 \mathrm{H}), 6.35(\mathrm{~d}, J=9.6 \mathrm{~Hz}, 1 \mathrm{H}), 6.27(\mathrm{~d}, J=2.4 \mathrm{~Hz}, 1 \mathrm{H}), 6.25(\mathrm{dd}, J=9.6,2.4$ $\mathrm{Hz}, 1 \mathrm{H}), 5.39(\mathrm{~d}, J=15.0 \mathrm{~Hz}, 1 \mathrm{H}), 5.32(\mathrm{~d}, J=15.0 \mathrm{~Hz}, 1 \mathrm{H}), 3.47(\mathrm{~d}, J=10.4$ 
$\mathrm{Hz}, 1 \mathrm{H}), 3.43(\mathrm{~d}, J=10.4 \mathrm{~Hz}, 1 \mathrm{H}), 2.91(\mathrm{~d}, J=17.4 \mathrm{~Hz}, 1 \mathrm{H}), 2.72(\mathrm{~d}, J=17.4$ $\mathrm{Hz}, 1 \mathrm{H}), 0.86(\mathrm{~s}, 9 \mathrm{H}) ;{ }^{13} \mathrm{C} \mathrm{NMR}\left(150 \mathrm{MHz}, \mathrm{CDCl}_{3}\right) \delta 187.8,172.0,153.5,140.2$, 139.6, 137.4, 136.4, 135.9, 129.4, 129.4, 129.1 (2C), 128.2, 128.1, 127.4, 127.3 (2C), 125.8, 123.9, 123.1, 121.2, 119.3, 110.5, 109.8, 54.7, 54.1, 50.7, 44.4, 42.9, 27.0 (3C); IR (ATR) 3029, 2970, 2927, 1746, 1680, 1625, 1550, 1455, 1415, $1218 \mathrm{~cm}^{-1}$; HRMS (ESI-TOF) $[\mathrm{M}+\mathrm{Na}]^{+}$calcd for $\mathrm{C}_{32} \mathrm{H}_{30} \mathrm{~N}_{2} \mathrm{NaO}_{2}{ }^{+} \mathrm{m} / z$ 497.2199, found $\mathrm{m} / \mathrm{z} 497.2210$.

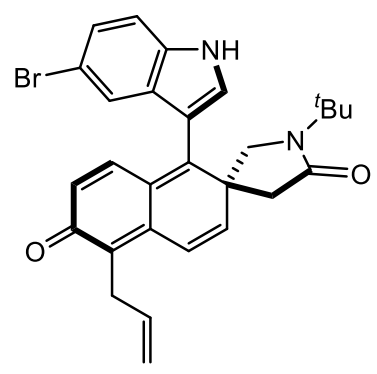

\section{5-Allyl-1-(5-bromo-1H-indol-3-yl)-1'-(tert-butyl)-6H-spiro[naphthalene-2,3'- pyrrolidine]-5',6-dione (9k)}

Prepared according to the general procedure B using 5-bromoindole and $\mathbf{1 b}$ which was added slowly over $0.5 \mathrm{~h}$ via a syringe pump at room temperature, and isolated as yellow solid $\left(42.2 \mathrm{mg}, 84 \%\right.$ yield). m.p. $187-189{ }^{\circ} \mathrm{C} ; \mathrm{R}_{f}=0.4$ ( $n$-hexane/EtOAc, $1 / 3) ;{ }^{1} \mathrm{H} \mathrm{NMR}\left(400 \mathrm{MHz} \mathrm{CDCl}_{3}\right) \delta 9.78$ (br s, 1H), 7.43 (s, $1 \mathrm{H}), 7.35(\mathrm{~d}, J=4.0 \mathrm{~Hz}, 1 \mathrm{H}), 7.35(\mathrm{~d}, J=2.8 \mathrm{~Hz}, 1 \mathrm{H}), 7.29(\mathrm{~d}, J=2.8 \mathrm{~Hz}, 1 \mathrm{H})$, $6.98(\mathrm{~d}, J=10.4 \mathrm{~Hz}, 1 \mathrm{H}), 6.93(\mathrm{~d}, J=10.0 \mathrm{~Hz}, 1 \mathrm{H}), 6.34(\mathrm{~d}, J=10.0 \mathrm{~Hz}, 1 \mathrm{H})$, $6.28(\mathrm{~d}, J=10.4 \mathrm{~Hz}, 1 \mathrm{H}), 5.90(\mathrm{ddt}, J=17.2,10.0,6.4 \mathrm{~Hz}, 1 \mathrm{H}), 5.09(\mathrm{dd}, J=$ $17.2,2.4 \mathrm{~Hz}, 1 \mathrm{H}), 5.05(\mathrm{dd}, J=10.0,2.4 \mathrm{~Hz}, 1 \mathrm{H}), 3.53(\mathrm{~d}, J=10.8 \mathrm{~Hz}, 1 \mathrm{H})$, $3.46(\mathrm{~d}, J=6.4 \mathrm{~Hz}, 2 \mathrm{H}), 3.44(\mathrm{~d}, J=10.8 \mathrm{~Hz}, 1 \mathrm{H}), 2.84(\mathrm{~d}, J=18.4 \mathrm{~Hz}, 1 \mathrm{H})$, $2.80(\mathrm{~d}, J=18.4 \mathrm{~Hz}, 1 \mathrm{H}), 0.84(\mathrm{~s}, 9 \mathrm{H}) ;{ }^{13} \mathrm{C} \mathrm{NMR}\left(100 \mathrm{MHz}, \mathrm{CDCl}_{3}\right) \delta 186.4$, 172.5, 151.1, 139.4 136.5, 135.7, 134.8, 134.4, 131.7, 130.4, 129.0, 128.7, 126.1, 124.6, 123.3, 121.3, 115.6, 114.3, 113.5, 110.3, 55.0, 54.2, 43.6, 28.8, 27.8, 27.0 (3C); IR (ATR) 3214, 2979, 2922, 1667, 1617, 1548, 1419, 1361, $1210 \mathrm{~cm}^{-1}$; HRMS (ESI-TOF) $[\mathrm{M}+\mathrm{Na}]^{+}$calcd for $\mathrm{C}_{28} \mathrm{H}_{27} \mathrm{BrN}_{2} \mathrm{NaO}_{2}{ }^{+} \mathrm{m} / z 525.1148$, found $m / z 525.1172$. 


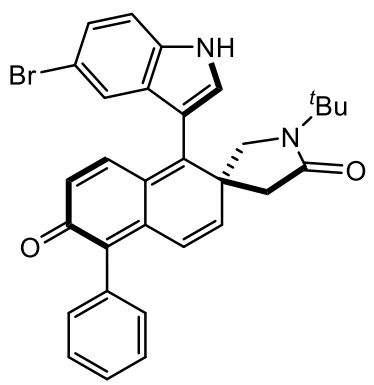

\section{1-(5-Bromo-1H-indol-3-yl)-1'-(tert-butyl)-5-phenyl-6H-spiro[naphthalene-}

\section{2,3'-pyrrolidine]-5',6-dione (91)}

Prepared according to the general procedure B using 5-bromoindole and 1c which was added slowly over $0.5 \mathrm{~h}$ via a syringe pump at room temperature, and isolated as pale orange solid ( $40.2 \mathrm{mg}, 74 \%$ yield).: m.p. $185-186{ }^{\circ} \mathrm{C} ; \mathrm{R}_{f}=$ 0.4 ( $n$-hexane/EtOAc, 1/3); ${ }^{1} \mathrm{H}$ NMR (600 MHz, $\left.\mathrm{CDCl}_{3}\right) \delta 10.07$ (br s, $\left.1 \mathrm{H}\right), 7.49$ (s, 1H), $7.46(\mathrm{dd}, J=6.0,7.2 \mathrm{~Hz}, 2 \mathrm{H}), 7.40-7.32(\mathrm{~m}, 4 \mathrm{H}), 7.26(\mathrm{~s}, 2 \mathrm{H}), 0.85(\mathrm{~s}$, $9 \mathrm{H}), 7.03(\mathrm{~d}, J=9.6 \mathrm{~Hz}, 1 \mathrm{H}), 6.65(\mathrm{~d}, J=9.6 \mathrm{~Hz}, 1 \mathrm{H}), 6.37(\mathrm{~d}, J=9.6 \mathrm{~Hz}, 1 \mathrm{H})$, $6.22(\mathrm{~d}, J=9.6 \mathrm{~Hz}, 1 \mathrm{H}), 3.51(\mathrm{~d}, J=10.8 \mathrm{~Hz}, 1 \mathrm{H}), 3.46(\mathrm{~d}, J=10.8 \mathrm{~Hz}, 1 \mathrm{H})$, $2.89(\mathrm{~d}, J=17.4 \mathrm{~Hz}, 1 \mathrm{H}), 2.88(\mathrm{~d}, J=17.4 \mathrm{~Hz}, 1 \mathrm{H}) ;{ }^{13} \mathrm{C} \mathrm{NMR}\left(150 \mathrm{MHz}, \mathrm{CDCl}_{3}\right)$ $\delta 186.3,172.4,152.8,139.5,136.8,135.0,135.0,134.5,134.4,130.7,130.6$, 130.3, 129.4, 128.6, 128.4, 128.3, 128.0, 126.0, 124.8, 124.6, 121.2, 114.3, 113.7, 110.0, 55.0, 54.3, 43.9, 43.4, 27.0 (3C); IR (ATR) 3238, 2972, 2923, 2864, 1745, 1669, 1622, 1541, 1456, 1415, 1362, $1215 \mathrm{~cm}^{-1}$; HRMS (ESI-TOF) $[\mathrm{M}+\mathrm{Na}]^{+}$ calcd for $\mathrm{C}_{31} \mathrm{H}_{27} \mathrm{BrN}_{2} \mathrm{NaO}_{2}{ }^{+} \mathrm{m} / z$ 561.1148, found $\mathrm{m} / z$ 561.1135.

\section{Preparation of 10a}

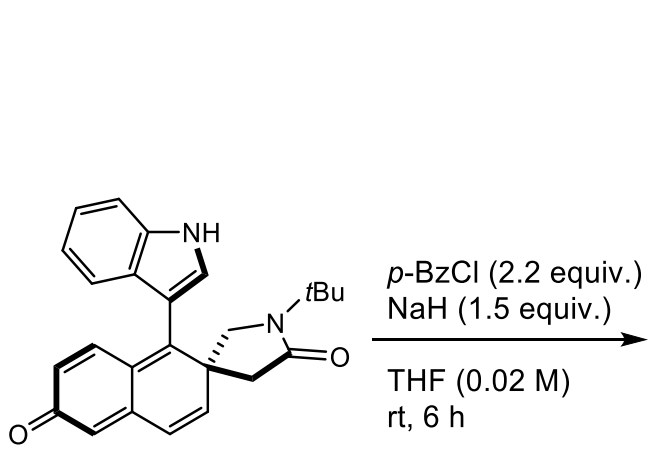

$9 a$

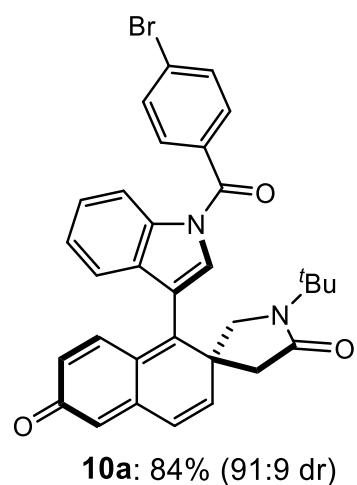

10a: $84 \%(91: 9 \mathrm{dr})$

A pre-dried $30 \mathrm{~mL}$ eggplant-shaped flask equipped with a magnetic stir bar was charged with 9a (19.2 $\mathrm{mg}, 0.05 \mathrm{mmol})$, which was subsequently 
dissolved in THF (2.5 mL) under an argon gas atmosphere. NaH (5 mg, 0.125 mmol, 1.5 equiv.) was added to the solution at $0{ }^{\circ} \mathrm{C}$ in an ice bath, and the reaction mixture was stirred for $0.5 \mathrm{~h}$ at room temperature. The resulting solution was cooled to $0{ }^{\circ} \mathrm{C}$ in an ice bath, para-bromo-benzoyl chloride $(24.2$ mg, $0.11 \mathrm{mmol}, 2.2$ equiv.) was then added, and stirring was continued for 6 $\mathrm{h}$ at room temperature. The reaction was quenched with the addition of water. The aqueous solution was extracted with EtOAc $\times 2$. The combined organic layers were washed with brine, dried over $\mathrm{Na}_{2} \mathrm{SO}_{4}$, concentrated under reduced pressure. The obtained crude residue was purified by flash column chromatography (column condition; diol $\mathrm{SiO}_{2}$, gradient elution: $n^{-}$ hexane/EtOAc $=3 / 1 \rightarrow 2 / 1$ ) to give $10 \mathrm{a}$ as yellow solid (23.8 $\mathrm{mg}$, 84\% yield).

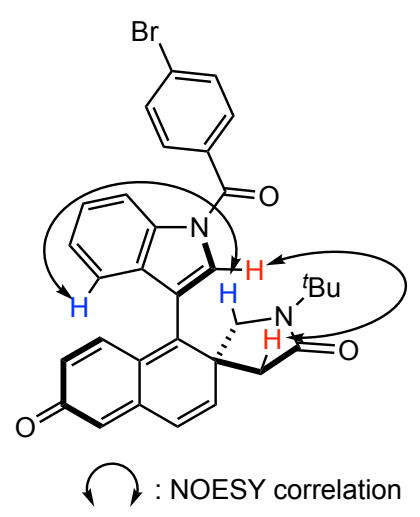

1-(1-(4-Bromobenzoyl)-1H-indol-3-yl)-1'-(tert-butyl)-6H-spiro[naphthalene2,3'-pyrrolidine]-5',6-dione (10a)

m.p. $157-159{ }^{\circ} \mathrm{C} ; \mathrm{R}_{f}=0.4$ ( $n$-hexane/EtOAc, $\left.1 / 3\right) ;{ }^{1} \mathrm{H} \mathrm{NMR}\left(600 \mathrm{MHz}, \mathrm{CDCl}_{3}\right.$ ) $\delta 8.54(\mathrm{~d}, J=7.8 \mathrm{~Hz}, 1 \mathrm{H}), 7.81(\mathrm{~d}, J=9.0 \mathrm{~Hz}, 2 \mathrm{H}), 7.75(\mathrm{~d}, J=9.0 \mathrm{~Hz}, 2 \mathrm{H})$, $7.51(\mathrm{dd}, J=7.8,7.2 \mathrm{~Hz}, 1 \mathrm{H}), 7.39(\mathrm{dd}, J=7.8,7.2 \mathrm{~Hz}, 1 \mathrm{H}), 7.35(\mathrm{~s}, 1 \mathrm{H}), 7.33$ $(\mathrm{d}, J=7.8 \mathrm{~Hz}, 1 \mathrm{H}), 6.89(\mathrm{~d}, J=9.6 \mathrm{~Hz}, 1 \mathrm{H}), 6.65(\mathrm{~d}, J=9.6 \mathrm{~Hz}, 1 \mathrm{H}), 6.30(\mathrm{~d}, J$ $=9.6 \mathrm{~Hz}, 1 \mathrm{H}), 6.27(\mathrm{~d}, J=1.2 \mathrm{~Hz}, 1 \mathrm{H}), 6.23(\mathrm{dd}, J=9.6,1.2 \mathrm{~Hz}, 1 \mathrm{H}), 3.56(\mathrm{~d}$, $J=10.4 \mathrm{~Hz}, 1 \mathrm{H}), 3.53(\mathrm{~d}, J=10.4 \mathrm{~Hz}, 1 \mathrm{H}), 2.80(\mathrm{~d}, J=17.4 \mathrm{~Hz}, 1 \mathrm{H}), 2.64(\mathrm{~d}$, $J=17.4 \mathrm{~Hz}, 1 \mathrm{H}), 0.84(\mathrm{~s}, 9 \mathrm{H}) ;{ }^{13} \mathrm{C} \mathrm{NMR}\left(150 \mathrm{MHz}, \mathrm{CDCl}_{3}\right) \delta 187.5,172.0$, $167.9,149.6,139.6,138.9,136.0,135.7,132.4$ (2C), 132.4, 131.5, 131.3 (2C), $130.2,128.7,127.7,126.6,126.5,125.5,125.4,124.5,119.2,117.3,116.5,54.5$, 54.1, 44.3, 43.3, 27.0 (3C); IR (ATR) 2976, 1690, 1670, 1626, 1585, 1450, 1362, 1332, 1261, $1224 \mathrm{~cm}^{-1}$; HRMS (ESI-TOF) $[\mathrm{M}+\mathrm{Na}]^{+}$calcd for 
Supporting Information

$\mathrm{C}_{32} \mathrm{H}_{27} \mathrm{BrN}_{2} \mathrm{NaO}_{3}{ }^{+} \mathrm{m} / z$ 589.1097, found $\mathrm{m} / z$ 589.1105. 


\section{X-ray Crystallographic Data}

ORTEP of 10a, CCDC No. 2031608

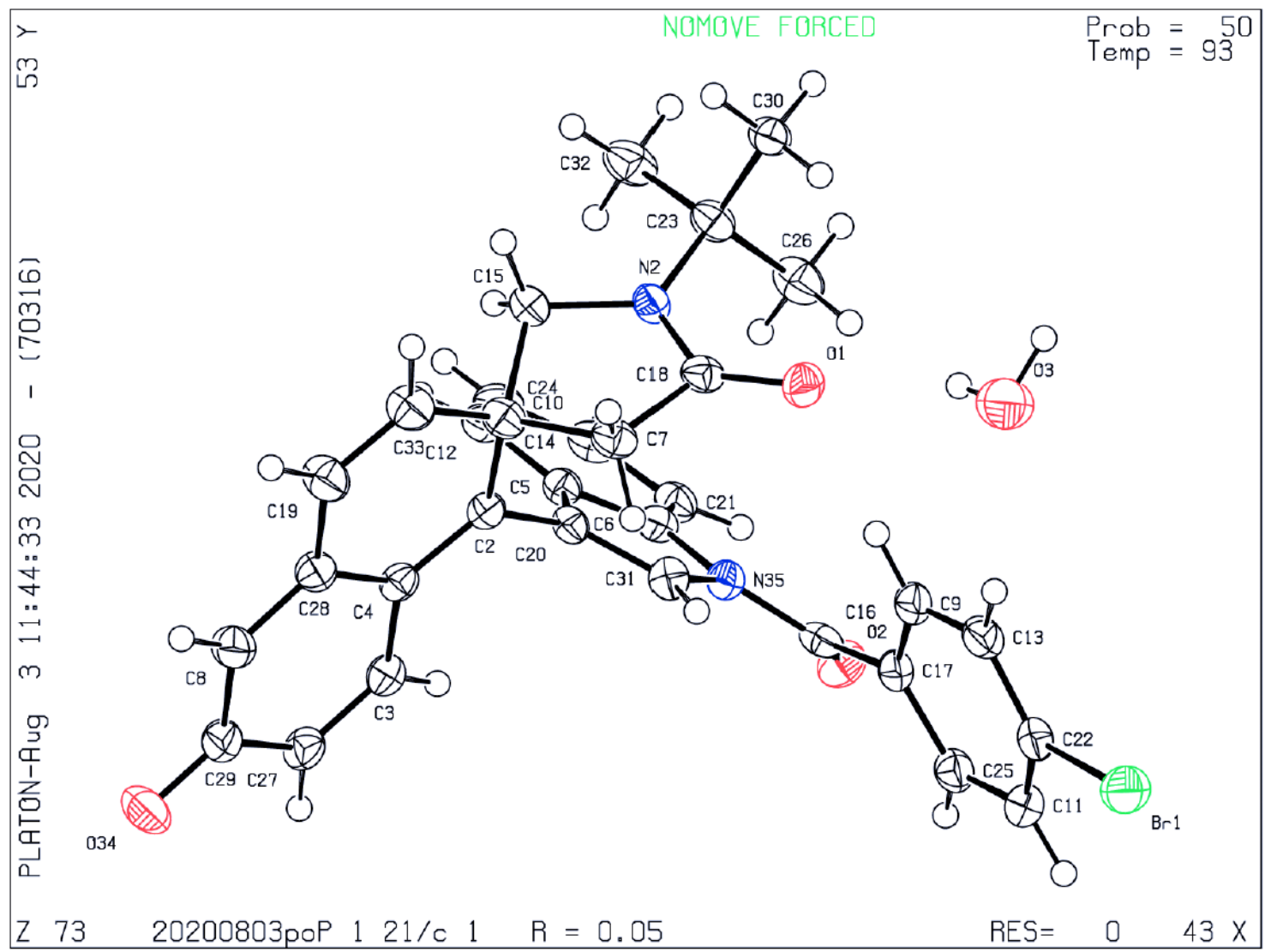

The ellipsoid contour probability level in the ORTEP is 50\%. Experimental

\section{Data Collection}

A colorless block crystal of $\mathrm{C} 32 \mathrm{H} 29 \mathrm{BrN} 2 \mathrm{O} 4$ having approximate dimensions of $0.200 \times 0.200 \times 0.100 \mathrm{~mm}$ was mounted on a glass fiber. All measurements were made on a Rigaku R-AXIS RAPID diffractometer using multi-layer mirror monochromated $\mathrm{Cu}-\mathrm{K} \alpha$ radiation.

The crystal-to-detector distance was $127.40 \mathrm{~mm}$. 
Cell constants and an orientation matrix for data collection corresponded to a primitive monoclinic cell with dimensions:

$$
\begin{aligned}
& \mathrm{a}=7.77343(14) \AA \\
& \mathrm{b}=13.7756(3) \AA \\
& \mathrm{c}=24.8433(5) \AA \\
& \mathrm{V}=2660.22(8) \AA^{3}
\end{aligned}
$$

For $\mathrm{Z}=4$ and F.W. $=585.50$, the calculated density is $1.462 \mathrm{~g} / \mathrm{cm}^{3}$. The reflection conditions of:

$$
\begin{array}{ll}
\text { h0l: } & \mathrm{l}=2 \mathrm{n} \\
\text { 0k0: } & \mathrm{k}=2 \mathrm{n}
\end{array}
$$

uniquely determine the space group to be:

$$
\mathrm{P} 2{ }_{1} / \mathrm{c}(\# 14)
$$

The data were collected at a temperature of $-180 \pm 1^{\circ} \mathrm{C}$ to a maximum $2 \theta$ value of $136.4^{\circ}$. A total of 45 oscillation images were collected. A sweep of data was done using $\omega$ scans from 80.0 to $260.0^{\circ}$ in $20.00^{\circ}$ step, at $\chi=54.0^{\circ}$ and $\phi=0.0^{\circ}$. The exposure rate was 6.0 [sec./O]. A second sweep was performed using $\omega$ scans from 80.0 to $260.0^{\circ}$ in $20.00^{\circ}$ step, at $\chi=54.0^{\circ}$ and $\phi$ $=90.0^{\circ}$. The exposure rate was 6.0 [sec./O]. Another sweep was performed using $\omega$ scans from 80.0 to $260.0^{\circ}$ in $20.00^{\circ}$ step, at $\chi=54.0^{\circ}$ and $\phi=180.0^{\circ}$. The exposure rate was 6.0 [sec./o]. Another sweep was performed using $\omega$ scans from 80.0 to $260.0^{\circ}$ in $20.00^{\circ}$ step, at $\chi=54.0^{\circ}$ and $\phi=270.0^{\circ}$. The exposure rate was 6.0 [sec./O]. Another sweep was performed using $\omega$ scans from 80.0 to $260.0^{\circ}$ in $20.00^{\circ}$ step, at $\chi=0.0^{\circ}$ and $\phi=0.0^{\circ}$. The exposure rate was 6.0 [sec./o]. The crystal-to-detector distance was $127.40 \mathrm{~mm}$. Readout was performed in the $0.100 \mathrm{~mm}$ pixel mode. 


\section{EXPERIMENTAL DETAILS}

The sample for X-ray crystal structure analysis was prepared by condensation method. A pre-dried $1 \mathrm{~mL}$ sample tube was charged with $10 \mathrm{a}$, which was dissolved in $\mathrm{CH}_{2} \mathrm{Cl}_{2}$. The sample tube was left to stand in a $5 \mathrm{~mL}$ sample tube charged with argon gas until a single crystal forms.

\section{A. Crystal Data}

Empirical Formula

Formula Weight

Crystal Color, Habit

Crystal Dimensions

Crystal System

Lattice Type

Lattice Parameters

Space Group
$\mathrm{C}_{32} \mathrm{H}_{29} \mathrm{BrN}_{2} \mathrm{O}_{4}$

585.50

colorless, block

$0.200 \times 0.200 \times 0.100 \mathrm{~mm}$

monoclinic

Primitive

$$
\begin{aligned}
& \mathrm{a}=7.77343(14) \AA \\
& \mathrm{b}=13.7756(3) \AA \\
& \mathrm{c}=24.8433(5) \AA \\
& \beta=90.465(6) \mathrm{o} \\
& \mathrm{V}=2660.22(8) \AA^{3}
\end{aligned}
$$

$\mathrm{P} 21_{1} / \mathrm{c}(\# 14)$ 
$\mathrm{D}_{\text {calc }}$

$1.462 \mathrm{~g} / \mathrm{cm}^{3}$

F000

1208.00

$\mu(\mathrm{CuK} \alpha)$

$24.469 \mathrm{~cm}^{-1}$

B. Intensity Measurements

Diffractometer

Radiation

monochromated

Voltage, Current

Temperature

Detector Aperture

Data Images

$\omega$ oscillation Range $(\chi=54.0, \phi=0.0)$

Exposure Rate

$\omega$ oscillation Range $(\chi=54.0, \phi=90.0)$
R-AXIS RAPID

$\operatorname{CuK} \alpha(\lambda=1.54187 \AA)$

multi-layer

mirror

$40 \mathrm{kV}, 30 \mathrm{~mA}$

$-180.0^{\circ} \mathrm{C}$

$460.0 \times 256.0 \mathrm{~mm}$

45 exposures

$80.0-260.0^{\circ}$

$6.0 \mathrm{sec} . /^{\mathrm{O}}$

$80.0-260.0^{\circ}$ 
Exposure Rate

$\omega$ oscillation Range $(\chi=54.0, \phi=180.0)$

Exposure Rate

$\omega$ oscillation Range $(\chi=54.0, \phi=270.0)$

Exposure Rate

$\omega$ oscillation Range $(\chi=0.0, \phi=0.0)$

Exposure Rate

Detector Position

Pixel Size

$2 \theta_{\max }$

No. of Reflections Measured

Corrections

$0.783)$
$6.0 \mathrm{sec} . / 0$

$80.0-260.0^{0}$

$6.0 \mathrm{sec} . / 0$

$80.0-260.0^{\circ}$

$6.0 \mathrm{sec} . / 0$

$80.0-260.0^{\circ}$

$6.0 \mathrm{sec} .{ }^{\circ}$

$127.40 \mathrm{~mm}$

$0.100 \mathrm{~mm}$

$136.4^{0}$

Total: 29736

Unique: $4860\left(\mathrm{R}_{\text {int }}=0.0614\right)$

Lorentz-polarization

Absorption

(trans. factors: 0.582

C. Structure Solution and Refinement 
Structure Solution

Refinement

Function Minimized

Least Squares Weights

$\left.2 \mathrm{Fc}^{2}\right) / 3$

$2 \theta_{\max }$ cutoff

Anomalous Dispersion

No. Observations (All reflections)

No. Variables

Reflection/Parameter Ratio

Residuals: R1 (I>2.00б(I))

Residuals: R (All reflections)

Residuals: wR2 (All reflections)

Goodness of Fit Indicator

Max Shift/Error in Final Cycle

Maximum peak in Final Diff. Map
Direct Methods

Full-matrix least-squares on $\mathrm{F}^{2}$

$$
\begin{aligned}
& \sum \mathrm{w}\left(\mathrm{Fo}^{2}-\mathrm{Fc}^{2}\right)^{2} \\
& \mathrm{w}=1 /\left[\sigma^{2}\left(\mathrm{Fo}^{2}\right)+(0.0461 \cdot \mathrm{P})^{2}\right. \\
& +2.4256 \cdot \mathrm{P}] \\
& \text { where } \mathrm{P}=\left(\operatorname{Max}\left(\mathrm{Fo}_{0}^{2}, 0\right)+\right.
\end{aligned}
$$

$136.4^{\mathrm{O}}$

All non-hydrogen atoms

4860

360

13.50

0.0501

0.0801

0.1172

1.047

0.000

$0.82 \mathrm{e}^{-} / \mathrm{A}^{3}$ 
Supporting Information

Minimum peak in Final Diff. Map

$-0.57 \mathrm{e}^{-} / \mathrm{A}^{3}$ 


\section{Additional Studies on $\mathrm{F}^{-}$Anion Sensor}

\section{Figure S1. Additional Colorimetric Experiments}

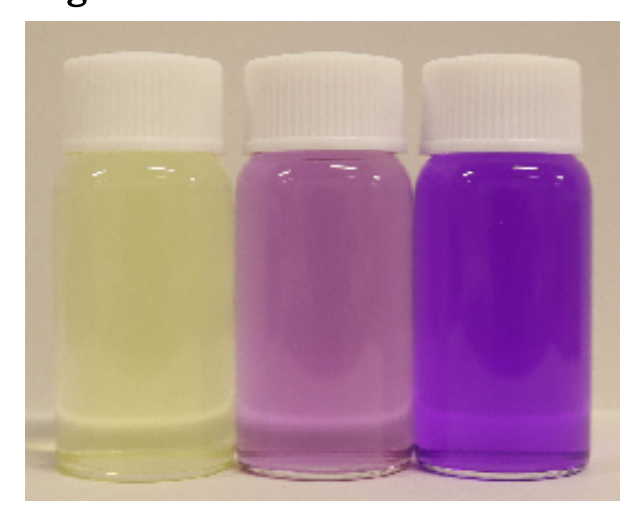

free (9a only) $\quad F^{-}$(1 eq.) $\quad F^{-}$(10 eq.)

conditions: 9a (0.19 mg, $0.5 \mu \mathrm{mol})$, TBAF ( $1 \mathrm{M}$ in THF) $(0.5 \mu \mathrm{L}, 0.5 \mu \mathrm{mol}, 1$ equiv. or $5 \mu \mathrm{L}, 5$ mol, 10 equiv.), $\mathrm{MeCN}(10 \mathrm{~mL}, 0.05 \mathrm{mM})$

\section{Figure S2. Additional UV/vis Spectra Experiments}

UV/vis spectra of 9a recorded in MeCN (0.017 mM) after addition of TBAF (1 $\mathrm{M}$ in THF, 0-6 equiv.).

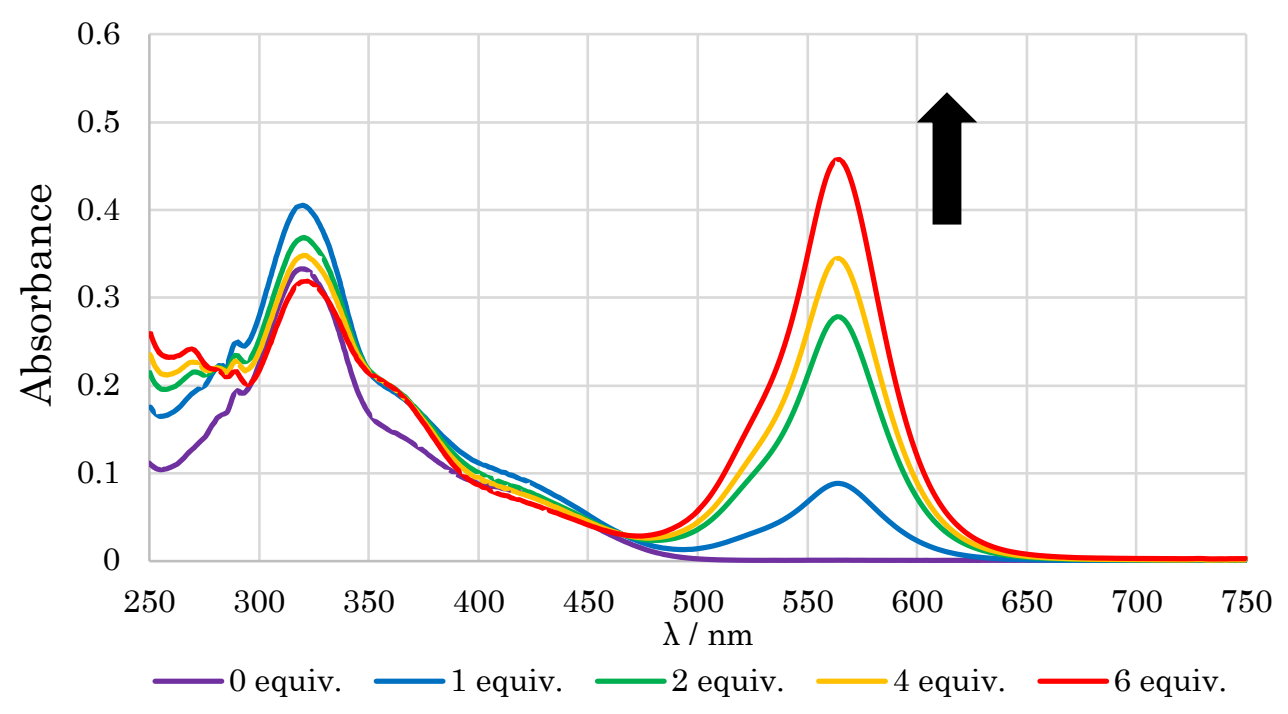


Supporting Information

\section{Figure S3. ${ }^{1} \mathrm{H}$ NMR Experiments}

Samples (1.9 mg, $0.005 \mathrm{mmol})$ were dissolved in acetonitrile-d6 $(1 \mathrm{~mL}, 5 \mathrm{mM})$. $\mathrm{TBAF} \cdot 3 \mathrm{H}_{2} \mathrm{O}(15.8 \mathrm{mg}, 10$ equiv. $)$ was used as $\mathrm{F}$ anion.
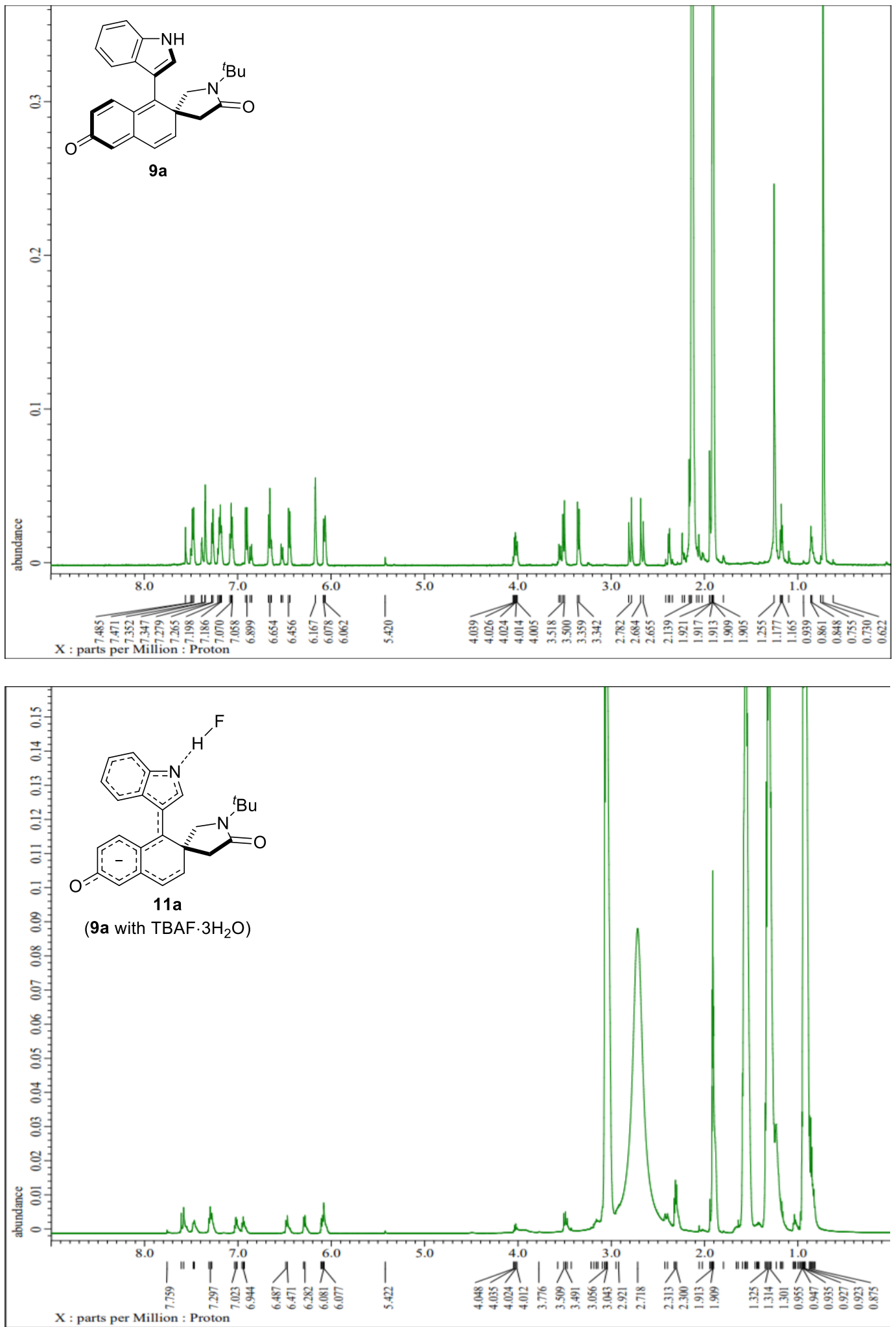

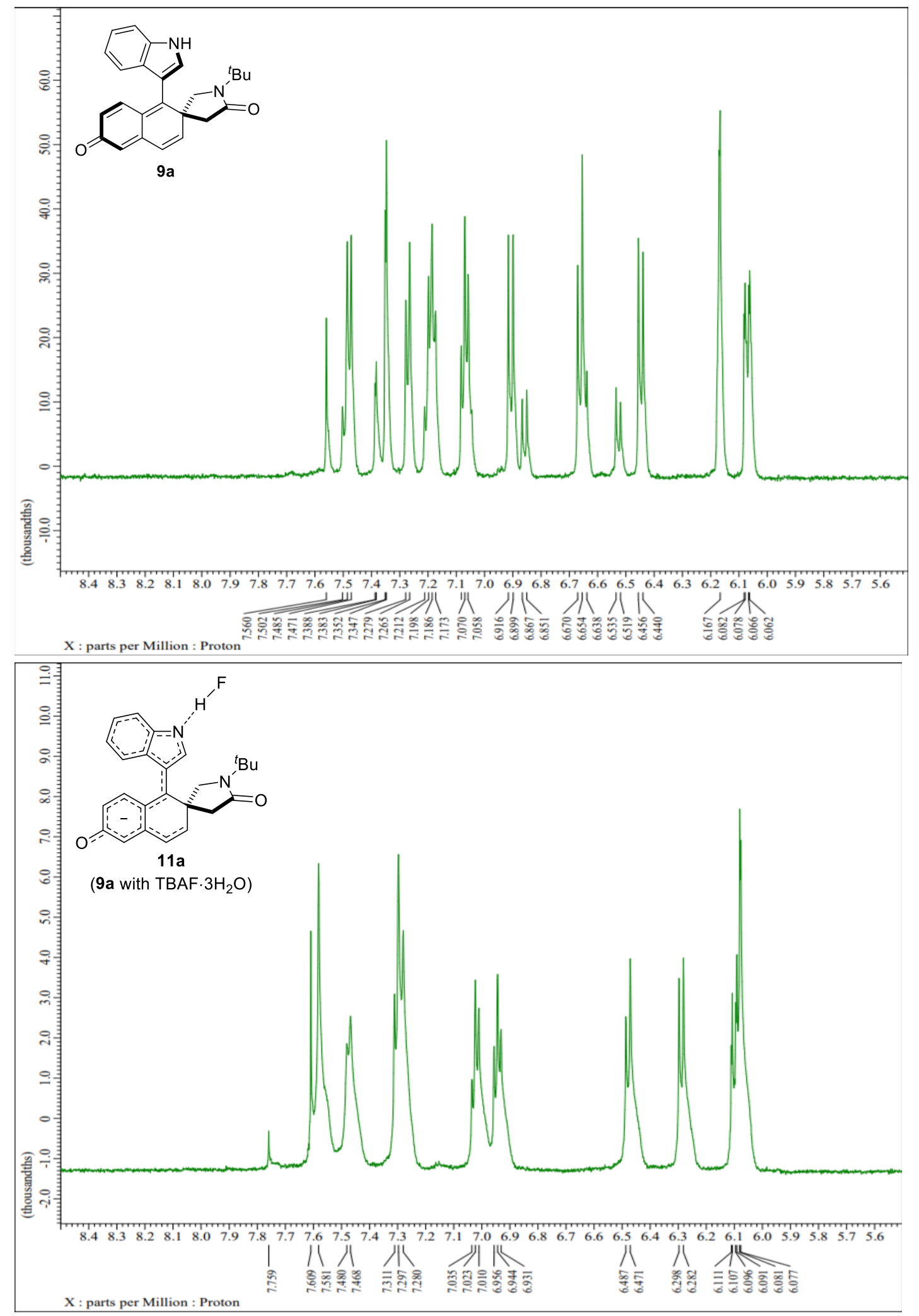


\section{Synthesis and Characterization of Substrates}

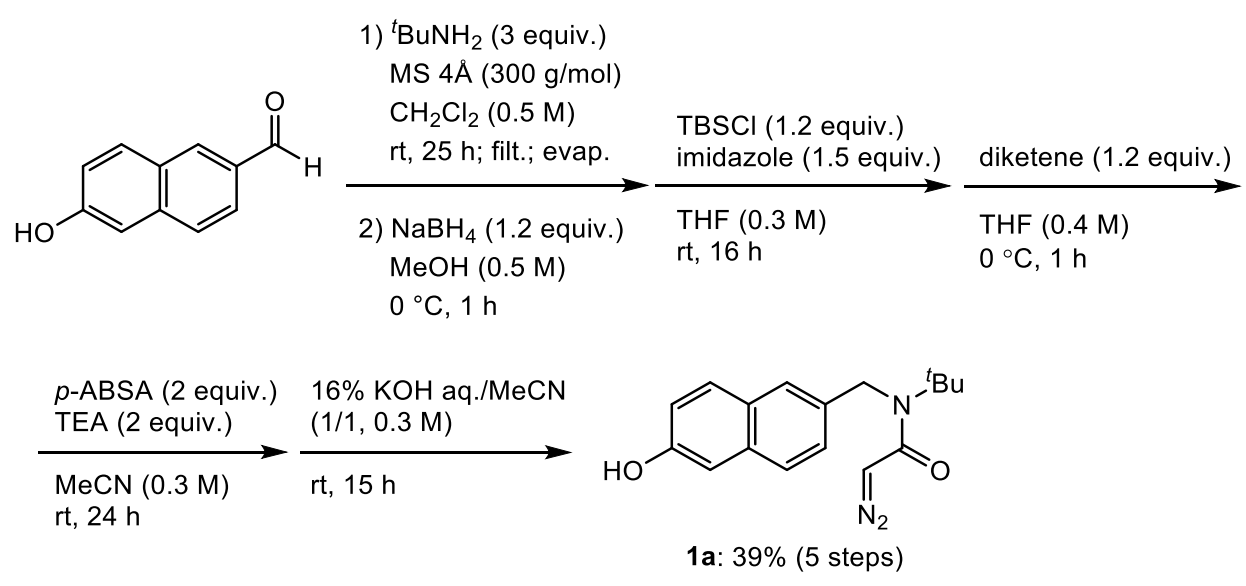

To a stirred suspension of 6-hydroxy-2-naphthaldehyde ( $860.9 \mathrm{mg}, 5 \mathrm{mmol})$ and activated MS $4 \AA(1500 \mathrm{mg}, 300 \mathrm{~g} / \mathrm{mol})$ in $\mathrm{CH}_{2} \mathrm{Cl}_{2}(10 \mathrm{~mL})$ was added ${ }^{t} \mathrm{BuNH}_{2}(1.6 \mathrm{~mL}, 15 \mathrm{mmol}, 3$ equiv.), and the reaction mixture was stirred for $25 \mathrm{~h}$ at room temperature. The mixture was then filtered through Celite ${ }^{\circledR}$ and concentrated under reduced pressure to remove $\mathrm{CH}_{2} \mathrm{Cl}_{2}$. $\mathrm{MeOH}(10 \mathrm{~mL})$ was added to the residue. The resulting solution was cooled to $0{ }^{\circ} \mathrm{C}$ in an ice bath, $\mathrm{NaBH}_{4}$ (227 mg, $6 \mathrm{mmol}, 1.2$ equiv.) was then added, and stirring was continued for $1 \mathrm{~h}$ at $0{ }^{\circ} \mathrm{C}$. The reaction was quenched with the addition of water and concentrated under reduced pressure to remove most of the $\mathrm{MeOH}$. The aqueous solution was extracted with $\mathrm{EtOAc} \times 3$, and the combined organic layers were washed with brine, dried over $\mathrm{Na}_{2} \mathrm{SO}_{4}$, and concentrated under reduced pressure to afford crude secondary amine $(750.4 \mathrm{mg})$, which was used for the next step without further purification.

To a stirred solution of crude secondary amine and imidazole (333.6 mg, 4.9 mmol, 1.5 equiv.) in THF (11 mL) was added TBSCl (587.8 mg, $3.9 \mathrm{mmol}, 1.2$ equiv.) at $0{ }^{\circ} \mathrm{C}$ in an ice bath. After being stirred for $16 \mathrm{~h}$ at room temperature, saturated aqueous $\mathrm{NH}_{4} \mathrm{Cl}$ was added. The reaction mixture was extracted with EtOAc $\times 3$, washed with brine, dried over $\mathrm{Na}_{2} \mathrm{SO}_{4}$, and concentrated under reduced pressure to afford the crude mixture, which was used for the next step without further purification.

To a stirred solution of the crude residue obtained above in THF $(8.3 \mathrm{~mL})$ was added diketene $(0.31 \mathrm{~mL}, 4.0 \mathrm{mmol}, 1.2$ equiv. $)$ at $0{ }^{\circ} \mathrm{C}$ in an ice bath, and 
the reaction mixture was stirred for $1 \mathrm{~h}$ at $0{ }^{\circ} \mathrm{C}$. The reaction was quenched with the addition of $1 \mathrm{~N}$ aqueous $\mathrm{KHSO}_{4}$ and the aqueous layer was extracted with EtOAc $\times 2$. The combined organic layers were washed with brine, dried over $\mathrm{Na}_{2} \mathrm{SO}_{4}$, and concentrated under reduced pressure. The resulting residue was passed through a short pad of silica to remove polar compounds ( $n^{-}$ hexane/EtOAc $=3 / 1$ ). The filtrate was concentrated to give crude mixture, which was used for the next step without further purification.

The crude residue obtained above was dissolved in MeCN (11 mL), and paraacetamidobenzenesulfonyl azide (1585.6 mg, $6.6 \mathrm{mmol}, 2$ equiv.) and $\mathrm{Et}_{3} \mathrm{~N}$ (0.92 mL, $6.6 \mathrm{mmol}, 2$ equiv.) were added at $0{ }^{\circ} \mathrm{C}$ in an ice bath. After being stirred for $24 \mathrm{~h}$ at room temperature, the reaction mixture was quenched with the addition of saturated aqueous $\mathrm{NH}_{4} \mathrm{Cl}$ and the aqueous layer was extracted with EtOAc $\times 2$. The combined organic layers were washed with brine, dried over $\mathrm{Na}_{2} \mathrm{SO}_{4}$, and concentrated under reduced pressure. The resulting residue was passed through a short pad of silica to remove polar compounds $\left(n^{-}\right.$ hexane/EtOAc $=2 / 1$ ) and concentrated to give crude mixture (987.0 mg), which was used for the next step without further purification.

A solution of the crude residue obtained above in $16 \%$ aqueous $\mathrm{KOH} / \mathrm{CH}_{3} \mathrm{CN}$ $(1 / 1,7.4 \mathrm{~mL})$ was stirred for $15 \mathrm{~h}$ at room temperature. The reaction was quenched with the addition of saturated aqueous $\mathrm{NH}_{4} \mathrm{Cl}$ and diluted with EtOAc. The aqueous layer was extracted with EtOAc $\times 3$, and the combined organic layers were washed with brine, dried over $\mathrm{Na}_{2} \mathrm{SO}_{4}$, concentrated under reduced pressure, and purified by recrystallization ( $n$-hexane/ $\mathrm{CH}_{2} \mathrm{Cl}_{2}$ ) to afford diazocarbonyl compound $1 \mathrm{a}$ as yellow solid $(585.8 \mathrm{mg}, 39 \%$ yield (5 steps)).

$N^{-}$(tert-Butyl)-2-diazo- $N$-((6-hydroxynaphthalen-2-yl)methyl)acetamide (1a) m.p. $147-149^{\circ} \mathrm{C} ; \mathrm{R}_{f}=0.4$ ( $n$-hexane/EtOAc, $\left.1 / 3\right) ;{ }^{1} \mathrm{H}$ NMR $(400 \mathrm{MHz}$, DMSOd6) $\delta 9.71(\mathrm{~s}, 1 \mathrm{H}), 7.76(\mathrm{~d}, J=8.8 \mathrm{~Hz}, 1 \mathrm{H}), 7.68(\mathrm{~d}, J=8.8 \mathrm{~Hz}, 1 \mathrm{H}), 7.55(\mathrm{~s}$, $1 \mathrm{H}), 7.26(\mathrm{~d}, J=8.8 \mathrm{~Hz}, 1 \mathrm{H}), 7.10(\mathrm{~s}, 1 \mathrm{H}), 7.06(\mathrm{dd}, J=8.8,2.4 \mathrm{~Hz}, 1 \mathrm{H}), 5.62$ $(\mathrm{s}, 1 \mathrm{H}), 4.54(\mathrm{~s}, 2 \mathrm{H}), 1.39$ (s, 9H); ${ }^{13} \mathrm{C}$ NMR (150 MHz, DMSO-d6) $\delta$ 167.0, 155.2, 134.0, 133.7, 129.2, 127.7, 126.5, 124.6, 123.9, 118.9, 108.6, 57.7, 48.4, 48.2, 28.6 (3C); IR (ATR) 3157, 3095, 2960, 2098, 1632, 1575, 1415, 1393, 1365, 
1220, 1189, $1172 \mathrm{~cm}^{-1}$; HRMS (ESI-TOF) $[\mathrm{M}+\mathrm{Na}]^{+}$calcd for $\mathrm{C}_{17} \mathrm{H}_{19} \mathrm{~N}_{3} \mathrm{NaO}_{2}{ }^{+}$ $\mathrm{m} / \mathrm{z} 320.1369$, found $\mathrm{m} / \mathrm{z} 320.1363$.

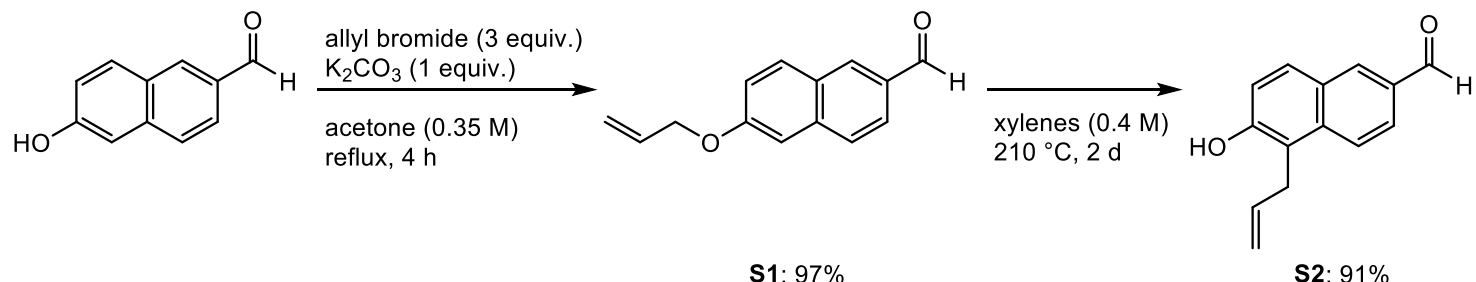

A pre-dried $100 \mathrm{~mL}$ eggplant-shaped flask equipped with a magnetic stir bar was charged with 6-hydroxy-2-naphthaldehyde (1721.8 $\mathrm{mg}, 10 \mathrm{mmol})$ and $\mathrm{K}_{2} \mathrm{CO}_{3}$ (1382.1 mg, $10 \mathrm{mmol}$, 1 equiv.), which were subsequently dissolved partially in acetone $(28.6 \mathrm{~mL})$ under an argon gas atmosphere. To the stirred suspension was added allyl bromide $(2.6 \mathrm{~mL}, 30 \mathrm{mmol}, 3$ equiv. $)$ at room temperature. The reaction mixture was refluxed for $4 \mathrm{~h}$ in an oil bath. The reaction mixture was then cooled to room temperature, diluted with $\mathrm{Et}_{2} \mathrm{O}$, and quenched with the addition of water. The aqueous layer was extracted with $\mathrm{Et}_{2} \mathrm{O} \times 2$ and the combined organic layers were dried over $\mathrm{Na}_{2} \mathrm{SO}_{4}$, filtered through a plug of cotton, and concentrated under reduced pressure. The resulting residue was purified by flash chromatography on silica gel ( $n$ hexane/EtOAc $=6 / 1)$ to afford $\mathbf{S} 1$ as beige solid $(2.1 \mathrm{~g}, 97 \%$ yield $)$.

A pre-dried $100 \mathrm{~mL}$ Schlenk tube equipped with a magnetic stir bar was charged with S1 (2.1 g, $9.7 \mathrm{mmol})$, which was subsequently dissolved in xylenes $(24.3 \mathrm{~mL})$ under an argon gas atmosphere. After being stirred for 2 days at $210^{\circ} \mathrm{C}$, the reaction mixture was then cooled to room temperature, and concentrated under reduced pressure. The aqueous layer was extracted with $\mathrm{Et}_{2} \mathrm{O} \times 2$ and the combined organic layers were dried over $\mathrm{Na}_{2} \mathrm{SO}_{4}$, filtered through a plug of cotton, and concentrated under reduced pressure. The resulting residue was purified by flash column chromatography ( $n$ hexane/EtOAc $=3 / 1)$ to afford $\mathbf{S} 2$ as green solid $(1.9 \mathrm{~g}, 91 \%$ yield $)$.

\section{6-(Allyloxy)-2-naphthaldehyde (S1)}

m.p. $56-58{ }^{\circ} \mathrm{C} ; \mathrm{R}_{f}=0.6$ ( $n$-hexane/EtOAc, $\left.3 / 1\right) ;{ }^{1} \mathrm{H} \mathrm{NMR}\left(400 \mathrm{MHz}, \mathrm{CDCl}_{3}\right) \delta$ 
$10.09(\mathrm{~s}, 1 \mathrm{H}), 8.24(\mathrm{~d}, J=1.6 \mathrm{~Hz}, 1 \mathrm{H}), 7.91(\mathrm{dd}, J=8.8,1.6 \mathrm{~Hz}, 1 \mathrm{H}), 7.89(\mathrm{~d}, J$ $=8.8 \mathrm{~Hz}, 1 \mathrm{H}), 7.78(\mathrm{~d}, J=8.8 \mathrm{~Hz}, 1 \mathrm{H}), 7.26(\mathrm{dd}, J=8.8,2.4 \mathrm{~Hz}, 1 \mathrm{H}), 7.18(\mathrm{~d}$, $J=2.4 \mathrm{~Hz}, 1 \mathrm{H}), 6.12(\mathrm{ddt}, J=17.2,10.4,5.6 \mathrm{~Hz}, 1 \mathrm{H}), 5.49(\mathrm{dd}, J=17.2,1.6$ $\mathrm{Hz}, 1 \mathrm{H}), 5.36$ (dd, $J=10.4,1.6 \mathrm{~Hz}, 1 \mathrm{H}), 4.69$ (d, $J=5.6 \mathrm{~Hz}, 2 \mathrm{H}) ;{ }^{13} \mathrm{C}$ NMR $(100$ $\left.\mathrm{MHz}_{2} \mathrm{CDCl}_{3}\right) \delta 192.2,159.3,138.3,134.4,132.7,132.5,131.3,128.1,127.9$, 123.7, 120.3, 118.4, 107.3, 69.1; IR (ATR) 2915, 2831, 2725, 2119, 1907, 1678, 1620, 1474, 1420, 1383, 1360, 1333, 1264, 1240, 1178, $1150 \mathrm{~cm}^{-1}$; HRMS (ESITOF) $[\mathrm{M}+\mathrm{Na}]^{+}$calcd for $\mathrm{C}_{14} \mathrm{H}_{12} \mathrm{NaO}_{2}{ }^{+} \mathrm{m} / z$ 235.0730, found $\mathrm{m} / z 235.0735$.

\section{5-Allyl-6-hydroxy-2-naphthaldehyde (S2)}

m.p. $122-124{ }^{\circ} \mathrm{C} ; \mathrm{R}_{f}=0.3$ ( $n$-hexane/EtOAc, $\left.3 / 1\right) ;{ }^{1} \mathrm{H} \mathrm{NMR}\left(400 \mathrm{MHz}, \mathrm{CDCl}_{3}\right)$ $\delta 10.11(\mathrm{~s}, 1 \mathrm{H}), 8.28(\mathrm{~d}, J=1.6 \mathrm{~Hz}, 1 \mathrm{H}), 7.99(\mathrm{~d}, J=9.2 \mathrm{~Hz}, 1 \mathrm{H}), 7.95(\mathrm{dd}, J=$ $9.2,1.6 \mathrm{~Hz}, 1 \mathrm{H}), 7.85(\mathrm{~d}, J=9.2 \mathrm{~Hz}, 1 \mathrm{H}), 7.22(\mathrm{~d}, J=9.2 \mathrm{~Hz}, 1 \mathrm{H}), 6.07$ (ddt, $J$ $=17.2,10.0,4.0 \mathrm{~Hz}, 1 \mathrm{H}), 5.60(\mathrm{~s}, 1 \mathrm{H}), 5.14(\mathrm{dd}, J=10.0,1.6 \mathrm{~Hz}, 1 \mathrm{H}), 5.06(\mathrm{dd}$, $J=17.2,1.6 \mathrm{~Hz}, 1 \mathrm{H}), 3.85(\mathrm{dd}, J=4.0,1.6 \mathrm{~Hz}, 2 \mathrm{H}) ;{ }^{13} \mathrm{C} \mathrm{NMR}\left(100 \mathrm{MHz}, \mathrm{CDCl}_{3}\right)$ $\delta 192.4,154.4,137.0,135.4,135.3,131.9,130.4,128.6,124.3,123.7,119.2$, 117.8, 116.5, 29.4; IR (ATR) 2970, 1747, 1670, 1488, 1426, 1365, 1285, 1230 $\mathrm{cm}^{-1} ;\left[\mathrm{M}+\mathrm{Na}+2 \mathrm{H}_{2} \mathrm{O}\right]+$ calcd for $\mathrm{C}_{14} \mathrm{H}_{16} \mathrm{NaO}_{4}{ }^{+} \mathrm{m} / z$ 271.0941, found $\mathrm{m} / z$ 271.0946.

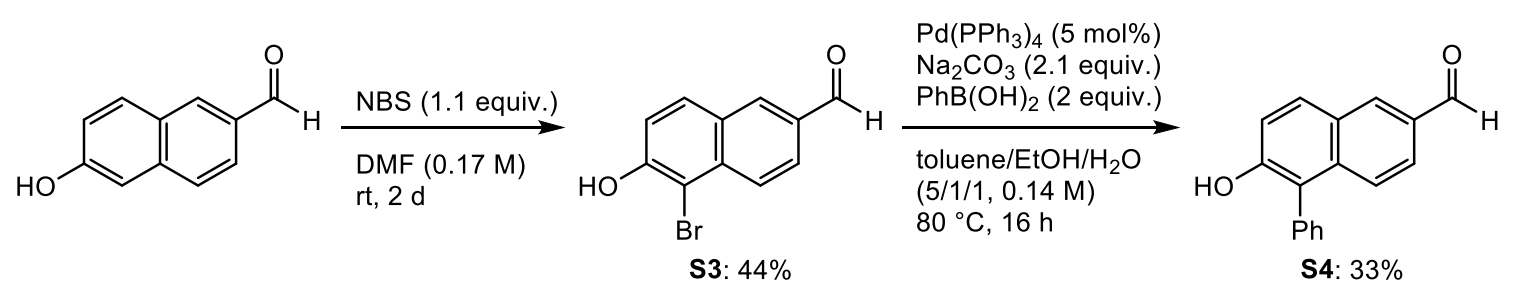

S3 was synthesized with reported procedure. ${ }^{[1]}$

A pre-dried $50 \mathrm{~mL}$ eggplant-shaped flask equipped with a magnetic stir bar was charged with toluene/EtOH/ $\mathrm{H}_{2} \mathrm{O}(5 / 1 / 1,14.3 \mathrm{~mL})$ and the solvents were degassed by bubbling with argon gas over $0.5 \mathrm{~h}$. To the stirred solution were added $\mathrm{Pd}\left(\mathrm{PPh}_{3}\right)_{4}(115.6 \mathrm{mg}, 0.1 \mathrm{mmol}, 5 \mathrm{~mol} \%), \mathrm{Na}_{2} \mathrm{CO}_{3}(22.3 \mathrm{mg}, 0.21 \mathrm{mmol}$, 2.1 equiv.), $\mathrm{S} 3$ (502.2 mg, $2 \mathrm{mmol}$ ), and $\mathrm{PhB}(\mathrm{OH})_{2}$ (607.8 $\mathrm{mg}, 4 \mathrm{mmol}, 2$ equiv.) at room temperature. After being stirred for $16 \mathrm{~h}$ at $80^{\circ} \mathrm{C}$, the reaction 
mixture was then cooled and diluted with water. The aqueous layer was extracted with EtOAc $\times 3$ and the combined organic layers were dried over $\mathrm{Na}_{2} \mathrm{SO}_{4}$, filtered through a plug of cotton, and concentrated under reduced pressure. The resulting residue was purified by flash column chromatography (gradient elution: toluene/ EtOAc $=15 / 1 \rightarrow 5 / 1)$ and recrystallization $\left(n^{-}\right.$ hexane/EtOAc) to afford as white solid S4 (162.3 mg, 33\% yield). ${ }^{1} \mathrm{H}$ and ${ }^{13} \mathrm{C}$ NMR, and other data are identical to those reported. ${ }^{[2]}$

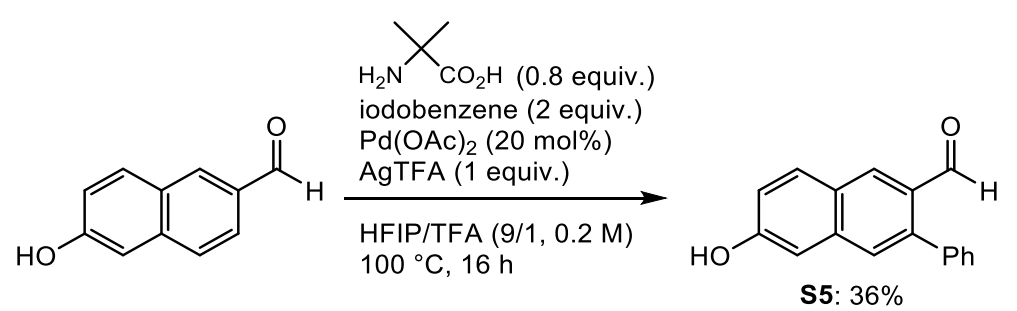

A $100 \mathrm{~mL}$ Schlenk tube equipped with a magnetic stir bar was charged with 6-hydroxy-2-naphthaldehyde ( $516.5 \mathrm{mg}, 3 \mathrm{mmol})$, iodobenzene $(0.67 \mathrm{~mL}, 6$ mmol, 2 equiv. $), \quad \operatorname{Pd}(\mathrm{OAc})_{2}(134.7 \mathrm{mg}, \quad 0.6 \mathrm{mmol}, 20 \mathrm{~mol} \%), \quad 2-$ aminoisobutyric acid (247.5 mg, $2.4 \mathrm{mmol}, 0.8$ equiv.), and AgTFA (662.6 mg, 3 mmol, 1 equiv.), which were subsequently dissolved in HFIP/TFA (9/1, $15 \mathrm{~mL}$ ) under an argon gas atmosphere. After being stirred for $16 \mathrm{~h}$ at $100{ }^{\circ} \mathrm{C}$, the reaction mixture was then cooled, diluted with EtOAc, filtrated through Celite $^{\circledR}$, and concentrated under reduced pressure. The resulting residue was purified by flash column chromatography ( $n$-hexane/EtOAc $=6 / 1)$ to afford $\mathbf{S 5}$ as white solid (270.6 mg, 36\% yield).

\section{6-Hydroxy-3-phenyl-2-naphthaldehyde (S5)}

m.p. $147-149{ }^{\circ} \mathrm{C} ; \mathrm{R}_{f}=0.1$ ( $n$-hexane/EtOAc, 5/1); ${ }^{1} \mathrm{H} \mathrm{NMR} \mathrm{(600} \mathrm{MHz,} \mathrm{CDCl}_{3}$ ) $\delta 10.06(\mathrm{~s}, 1 \mathrm{H}), 8.51(\mathrm{~s}, 1 \mathrm{H}), 7.95(\mathrm{~d}, J=9.6 \mathrm{~Hz}, 1 \mathrm{H}), 7.68(\mathrm{~s}, 1 \mathrm{H}), 7.51-7.48$ $(\mathrm{m}, 1 \mathrm{H}), 7.49(\mathrm{~d}, J=6.0 \mathrm{~Hz}, 1 \mathrm{H}), 7.46(\mathrm{~s}, 1 \mathrm{H}), 7.45^{-7.46}(\mathrm{~m}, 1 \mathrm{H}), 7.46(\mathrm{~d}, J=$ $6.0 \mathrm{~Hz}, 1 \mathrm{H}), 7.20-7.18(\mathrm{~m}, 2 \mathrm{H}), 5.51(\mathrm{br} \mathrm{s}, 1 \mathrm{H}) ;{ }^{13} \mathrm{C} \mathrm{NMR}\left(150 \mathrm{MHz}, \mathrm{CDCl}_{3}\right) \delta$ 192.7, 156.7, 141.8, 138.5, 137.5, 132.2, 130.2 (2C), 130.2, 130.0, 128.6 (2C), 128.2 128.0, 127.4, 119.5, 109.6; IR (ATR) 2970, 1746, 1661, 1610, 1430, 1396, 1366, 1282, 1229, $1145 \mathrm{~cm}^{-1}$; HRMS (ESI-TOF) $[\mathrm{M}+\mathrm{Na}]^{+}$calcd for $\mathrm{C}_{17} \mathrm{H}_{12} \mathrm{NaO}_{2}{ }^{+} \mathrm{m} / z$ 271.0730, found $\mathrm{m} / z 271.0735$. 

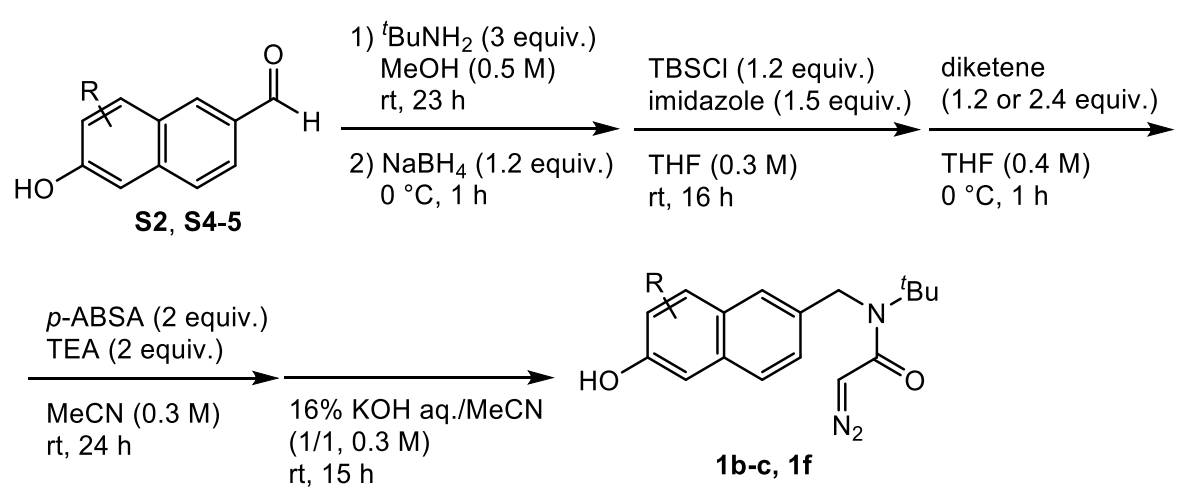

To a stirred solution of $\mathbf{S} 2$ or $\mathbf{S} 4-5$ in $\mathbf{M e O H}$ was added ${ }^{t} \mathrm{BuNH}_{2}$ (3 equiv.), and the reaction mixture was stirred for $23 \mathrm{~h}$ at room temperature. The reaction mixture was cooled to $0{ }^{\circ} \mathrm{C}$ in an ice bath, $\mathrm{NaBH}_{4}$ (1.2 equiv.) was then added, and stirring was continued for $1 \mathrm{~h}$ at $0{ }^{\circ} \mathrm{C}$. The reaction was quenched with the addition of water and concentrated under reduced pressure to remove most of the $\mathrm{MeOH}$. The aqueous solution was extracted with EtOAc $\times 3$, and the combined organic layers were washed with brine, dried over $\mathrm{Na}_{2} \mathrm{SO}_{4}$, and concentrated under reduced pressure to afford crude secondary amine, which was used for the next step without further purification.

1b-c and If were synthesized from secondary amine under the same conditions as synthesis of $1 \mathbf{a}$.<smiles>C=CCc1c(O)ccc2cc(CN(CC)C(=O)C=N)ccc12</smiles>

\section{$N^{-((5-A l l y l-6-h y d r o x y n a p h t h a l e n-2-y l) m e t h y l)-~} N$-(tert-butyl)-2-}

\section{diazoacetamide (1b)}

$5 \mathrm{mmol}$ of $\mathbf{S} 2$ and 2.4 equiv. of diketene were used and $1 \mathrm{~b}$ was afforded as pale yellow solid (197.2 mg, 12\% yield (5 steps)).: m.p. $133-135{ }^{\circ} \mathrm{C} ; \mathrm{R}_{f}=0.6\left(n^{-}\right.$ hexane/EtOAc, 1/1); ${ }^{1} \mathrm{H} \mathrm{NMR}\left(400 \mathrm{MHz} \mathrm{CDCl}_{3}\right) \delta 7.90(\mathrm{~d}, J=8.8 \mathrm{~Hz}, 1 \mathrm{H})$, $7.65(\mathrm{~d}, J=8.8 \mathrm{~Hz}, 1 \mathrm{H}), 7.60(\mathrm{~s}, 1 \mathrm{H}), 7.31(\mathrm{~d}, J=8.8 \mathrm{~Hz}, 1 \mathrm{H}), 7.15(\mathrm{~d}, J=8.8$ $\mathrm{Hz}, 1 \mathrm{H}), 6.07$ (ddt, $J=17.2,10.4,5.6 \mathrm{~Hz}, 1 \mathrm{H}), 5.37-5.51(\mathrm{~m}, 1 \mathrm{H}), 5.11(\mathrm{~d}, J=$ $10.4 \mathrm{~Hz}, 1 \mathrm{H}), 5.08$ (d, $J=17.2 \mathrm{~Hz}, 1 \mathrm{H}), 4.81(\mathrm{~s}, 1 \mathrm{H}), 4.57$ (s, 2H), 3.83 (d, $J=$ $5.6 \mathrm{~Hz}, 2 \mathrm{H}), 1.53(\mathrm{~s}, 9 \mathrm{H}) ;{ }^{13} \mathrm{C} \mathrm{NMR}\left(150 \mathrm{MHz} \mathrm{CDCl}_{3}\right) \delta 168.4,151.7,135.9$, 133.3, 132.7, 129.5, 128.1, 125.1, 124.4, 124.1, 118.8, 117.3, 116.0, 58.6, 49.2, 
49.0, 29.5, 29.2 (3C); IR (ATR) 3155, 2971, 2926, 2098, 1745, 1574, 1413, 1379, 1218, $1193 \mathrm{~cm}^{-1}$; HRMS (ESI-TOF) $[\mathrm{M}+\mathrm{Na}]^{+}$calcd for $\mathrm{C}_{20} \mathrm{H}_{23} \mathrm{~N}_{3} \mathrm{NaO}_{2}{ }^{+} \mathrm{m} / Z$ 360.1682 , found $\mathrm{m} / z$ 360.1686.<smiles>CCCCCN(Cc1ccc2c(-c3ccccc3)c(O)ccc2c1)C(=O)C#N</smiles>

\section{$N$-(tert-Butyl)-2-diazo- $N$-((6-hydroxy-5-phenylnaphthalen-2-} yl)methyl)acetamide (1c)

$1.8 \mathrm{mmol}$ of $\mathbf{S} 4$ was used and 1c was afforded as pale yellow solid (146.6 $\mathrm{mg}$, $33 \%$ yield (5 steps)). m.p. $225-227{ }^{\circ} \mathrm{C} ; \mathrm{R}_{f}=0.5$ ( $n$-hexane/EtOAc, $\left.1 / 1\right) ;{ }^{1} \mathrm{H}$ $\operatorname{NMR}\left(400 \mathrm{MHz} \mathrm{CDCl}_{3}\right) \delta 7.78(\mathrm{~d}, J=7.2 \mathrm{~Hz}, 1 \mathrm{H}), 7.63(\mathrm{~d}, J=7.2 \mathrm{~Hz}, 1 \mathrm{H})$, $7.60(\mathrm{dd}, J=6.8,6.0 \mathrm{~Hz}, 1 \mathrm{H}), 7.59(\mathrm{~d}, J=8.0 \mathrm{~Hz}, 1 \mathrm{H}), 7.53(\mathrm{ddd}, J=7.6,7.6$, $1.6 \mathrm{~Hz}, 1 \mathrm{H}), 7.43(\mathrm{~s}, 1 \mathrm{H}), 7.42(\mathrm{~d}, J=6.8 \mathrm{~Hz}, 1 \mathrm{H}), 7.40(\mathrm{~d}, J=6.0 \mathrm{~Hz}, 1 \mathrm{H}), 7.29$ (d, $J=9.2 \mathrm{~Hz}, 1 \mathrm{H}), 7.18(\mathrm{dd}, J=9.2,1.6 \mathrm{~Hz}, 1 \mathrm{H}), 5.15(\mathrm{a}, 1 \mathrm{H}), 4.79(\mathrm{~s}, 1 \mathrm{H})$, $4.55(\mathrm{~s}, 2 \mathrm{H}), 1.56(\mathrm{~s}, 9 \mathrm{H}) ;{ }^{13} \mathrm{C} \mathrm{NMR}\left(150 \mathrm{MHz} \mathrm{CDCl}_{3}\right) \delta 168.2,150.4,134.0$, 133.8, 132.6, 131.2 (2C), 129.9 (2C), 129.4, 129.0, 128.8, 125.7, 124.6, 124.5, 121.2, 118.2, 58.5, 49.0, 48.9, 29.1 (3C); IR (ATR) 3372, 3113, 2955, 2112, 1739, 1613, 1598, 1405, 1380, 1217, 1191, 1159, $1138 \mathrm{~cm}^{-1}$; HRMS (ESI-TOF) [M + $\mathrm{Na}]^{+}$calcd for $\mathrm{C}_{23} \mathrm{H}_{23} \mathrm{~N}_{3} \mathrm{NaO}_{2}{ }^{+} \mathrm{m} / z$ 396.1682, found $\mathrm{m} / z$ 396.1686.<smiles>NCC(=O)N(N)Cc1ccc2cc(O)ccc2c1</smiles>

$N$-(tert-Butyl)-2-diazo- $N$-((6-hydroxy-3-phenylnaphthalen-2yl)methyl)acetamide (1f)

$1.1 \mathrm{mmol}$ of $\mathbf{S} 5$ was used and $\mathbf{1 f}$ was afforded as pale pink solid (60.1 mg, 15\% yield (5 steps)).: m.p. $161-163{ }^{\circ} \mathrm{C} ; \mathrm{R}_{f}=0.3$ ( $n$-hexane/EtOAc, $3 / 1$ ); ${ }^{1} \mathrm{H} \mathrm{NMR}$ $\left(600 \mathrm{MHz} \mathrm{CDCl}_{3}\right) \delta 7.76(\mathrm{~d}, J=9.0 \mathrm{~Hz}, 1 \mathrm{H}), 7.71(\mathrm{~s}, 1 \mathrm{H}), 7.54(\mathrm{~s}, 1 \mathrm{H}), 7.49(\mathrm{t}$, $J=7.8 \mathrm{~Hz}, 1 \mathrm{H}), 7.47(\mathrm{~d}, J=7.8 \mathrm{~Hz}, 1 \mathrm{H}), 7.43(\mathrm{dd}, J=7.8,9.0 \mathrm{~Hz}, 1 \mathrm{H}), 7.32(\mathrm{~d}$, $J=7.8 \mathrm{~Hz}, 2 \mathrm{H}), 7.18(\mathrm{~s}, 1 \mathrm{H}), 7.17(\mathrm{dd}, J=8.4,2.4 \mathrm{~Hz}, 1 \mathrm{H}), 6.03(\mathrm{br} \mathrm{s}, 1 \mathrm{H})$, $4.80(\mathrm{~s}, 1 \mathrm{H}), 4.31(\mathrm{~s}, 2 \mathrm{H}), 1.46(\mathrm{~s}, 9 \mathrm{H}) ;{ }^{13} \mathrm{C} \mathrm{NMR}\left(150 \mathrm{MHz}, \mathrm{CDCl}_{3}\right) \delta 168.4$, 
154.5, 140.4, 139.2, 133.7, 131.6, 129.7, 128.9 (2C), 128.8 (2C), 128.2, 127.9, 127.8, 125.1, 118.7, 109.4, 58.5, 49.1, 48.4, 29.0 (3C); IR (ATR) 3220, 3099, 2956, 2925, 2109, 1746, 1588, 1415, 1372, $1212 \mathrm{~cm}^{-1}$; HRMS (ESI-TOF) [M + $\mathrm{Na}]^{+}$calcd for $\mathrm{C}_{23} \mathrm{H}_{23} \mathrm{~N}_{3} \mathrm{NaO}_{2}{ }^{+} \mathrm{m} / z$ 396.1682, found $\mathrm{m} / z$ 396.1684.
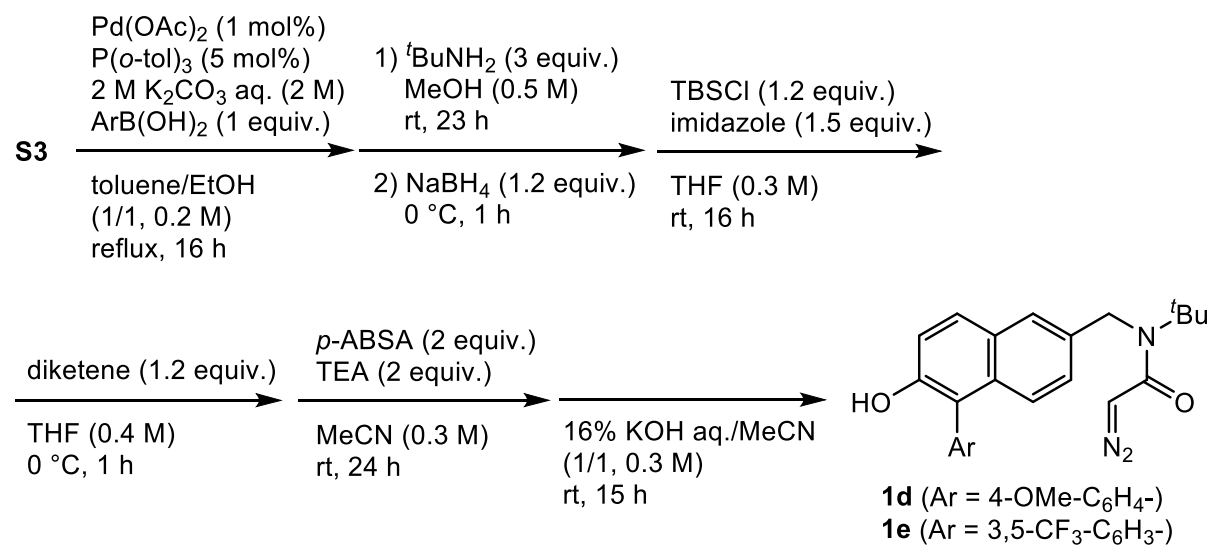

A $50 \mathrm{~mL}$ eggplant-shaped flask equipped with a magnetic stir bar was charged with $\mathbf{S} 3(749.9 \mathrm{mg}, 3 \mathrm{mmol}), \operatorname{ArB}(\mathrm{OH})_{2}(3 \mathrm{mmol}, 1$ equiv. $), \mathrm{P}(o \text {-tol })_{3}$ (45.7 $\mathrm{mg}, 0.15 \mathrm{mmol}, 5 \mathrm{~mol} \%$ ), and $2 \mathrm{M}$ aqueous $\mathrm{K}_{2} \mathrm{CO}_{3}(1.5 \mathrm{~mL})$, which were subsequently dissolved partially in toluene/EtOH $(1 / 1,15 \mathrm{~mL})$ under an argon gas atmosphere. After being refluxed for $16 \mathrm{~h}$, the reaction mixture was then cooled, diluted with EtOAc and water. The aqueous layer was extracted with EtOAc $\times 3$ and the combined organic layers were dried over $\mathrm{Na}_{2} \mathrm{SO}_{4}$, filtered through a plug of cotton, concentrated under reduced pressure, and passed through a short pad of silica to remove borane reagents ( $n$-hexane/EtOAc) to give the crude mixture.

$1 \mathrm{~d}$ and $1 \mathrm{e}$ were synthesized from the crude aldehyde with the same conditions as synthesis of $1 c$.<smiles>COc1ccc(-c2c(O)ccc3cc(CN(C(C)=O)C(=O)C=N)ccc23)cc1</smiles>

$N$-(tert-Butyl)-2-diazo- $N^{-}$((6-hydroxy-5-(4-methoxyphenyl)naphthalen-2yl)methyl)acetamide (1d) 
$3 \mathrm{mmol}$ of $\mathbf{S} 3$ was used and $\mathbf{1} \mathbf{d}$ was afforded as pale yellow solid $(77.3 \mathrm{mg}, 6 \%$ yield (6 steps)).: m.p. $199-201{ }^{\circ} \mathrm{C} ; \mathrm{R}_{f}=0.6$ ( $n$-hexane/EtOAc, $\left.1 / 1\right) ;{ }^{1} \mathrm{H} \mathrm{NMR}$ $\left(400 \mathrm{MHz}, \mathrm{CDCl}_{3}\right) \delta 7.76(\mathrm{~d}, J=8.8 \mathrm{~Hz}, 1 \mathrm{H}), 7.63(\mathrm{~s}, 1 \mathrm{H}), 7.42(\mathrm{~d}, J=8.8 \mathrm{~Hz}$, $1 \mathrm{H}), 7.34(\mathrm{~d}, J=8.8 \mathrm{~Hz}, 2 \mathrm{H}), 7.28(\mathrm{~d}, J=8.8 \mathrm{~Hz}, 1 \mathrm{H}), 7.17(\mathrm{~d}, J=8.8 \mathrm{~Hz}, 1 \mathrm{H})$, $7.13(\mathrm{~d}, J=8.8 \mathrm{~Hz}, 2 \mathrm{H}), 5.21(\mathrm{br} \mathrm{s}, 1 \mathrm{H}), 4.79(\mathrm{~s}, 1 \mathrm{H}), 4.55(\mathrm{~s}, 2 \mathrm{H}), 3.92(\mathrm{~s}, 3 \mathrm{H})$, $1.51(\mathrm{~s}, 9 \mathrm{H}) ;{ }^{13} \mathrm{C} \mathrm{NMR}\left(150 \mathrm{MHz}, \mathrm{CDCl}_{3}\right) \delta 168.2,160.0,150.7,133.7,133.0$, 132.4 (2C), 129.2, 129.1, 125.7 (2C), 124.6, 124.4, 120.9, 118.1, 115.3 (2C), 58.5, 55.6, 49.0, 49.0, 29.1 (3C); IR (ATR) 3296, 2970, 2107, 1739, 1596, 1515, 1406, 1379, $1217 \mathrm{~cm}^{-1}$; HRMS (ESI-TOF) [M + Na] ${ }^{+}$calcd for $\mathrm{C}_{24} \mathrm{H}_{25} \mathrm{~N}_{3} \mathrm{NaO}_{3}{ }^{+} \mathrm{m} / Z$ 426.1788 , found $\mathrm{m} / \mathrm{z} 426.1798$.

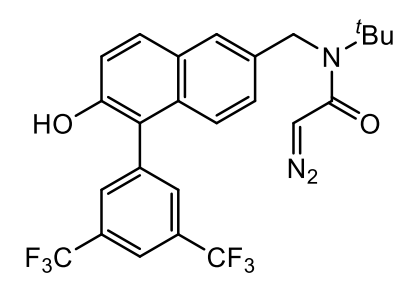

$N$-((5-(3,5-Bis(trifluoromethyl)phenyl)-6-hydroxynaphthalen-2-yl)methyl)- $N$ (tert-butyl)-2-diazoacetamide (1e)

$3 \mathrm{mmol}$ of $\mathbf{S} 3$ was used and 1e was afforded as pale yellow solid $(79.0 \mathrm{mg}, 6 \%$ yield (6 steps)).: m.p. $136-138{ }^{\circ} \mathrm{C} ; \mathrm{R}_{f}=0.6$ (n-hexane/EtOAc, $\left.1 / 1\right) ;{ }^{1} \mathrm{H}$ NMR $\left(600 \mathrm{MHz}, \mathrm{CDCl}_{3}\right) \delta 7.92(\mathrm{~s}, 2 \mathrm{H}), 7.81(\mathrm{~d}, J=9.0 \mathrm{~Hz}, 1 \mathrm{H}), 7.80(\mathrm{~s} 1 \mathrm{H}), 7.66(\mathrm{~s}$, $1 \mathrm{H}), 7.31(\mathrm{~d}, J=7.2 \mathrm{~Hz}, 1 \mathrm{H}), 7.30(\mathrm{~d}, J=9.0 \mathrm{~Hz}, 1 \mathrm{H}), 7.23(\mathrm{~d}, J=7.2 \mathrm{~Hz}, 1 \mathrm{H})$, $6.10(\mathrm{br} \mathrm{s}, 1 \mathrm{H}), 4.80(\mathrm{~s}, 1 \mathrm{H}), 4.57(\mathrm{~s}, 2 \mathrm{H}), 1.53(\mathrm{~s}, 9 \mathrm{H}) ;{ }^{13} \mathrm{C} \mathrm{NMR}(150 \mathrm{MHz}$, $\left.\mathrm{CDCl}_{3}\right) \delta$ 168.5, 151.0, 137.8, 134.1, 132.4 ( $\left.J=34.5 \mathrm{~Hz}, 2 \mathrm{C}\right), 132.3,131.7(2 \mathrm{C})$, $130.5,129.1,127.8,125.3,124.9,124.4,122.6,122.0,118.8,118.6,58.7,49.3$, 49.0, 29.2 (3C); ${ }^{19} \mathrm{~F} \mathrm{NMR} \mathrm{(564} \mathrm{MHz,} \mathrm{CDCl}_{3}$ ) $\delta$-62.6; IR (ATR) 3296, 2970, 2107, 1739, 1596, 1515, 1406, 1379, $1217 \mathrm{~cm}^{-1}$; HRMS (ESI-TOF) $[\mathrm{M}+\mathrm{Na}]^{+}$ calcd for $\mathrm{C}_{25} \mathrm{H}_{21} \mathrm{~F}_{6} \mathrm{~N}_{3} \mathrm{NaO}_{2}{ }^{+} \mathrm{m} / z$ 532.1430, found $\mathrm{m} / z$ 532.1437. 

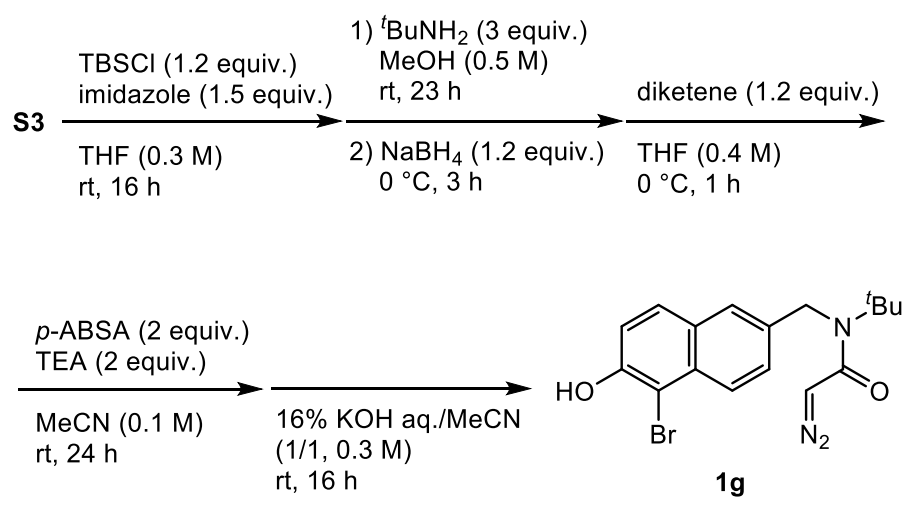

To a stirred solution of S3 (2510.8 $\mathrm{mg}, 10 \mathrm{mmol})$ and imidazole (1021.2 $\mathrm{mg}$, $15 \mathrm{mmol}, 1.5$ equiv.) in THF (33.3 mL) was added TBSCl (1808.6 mg, $12 \mathrm{mmol}$, 1.2 equiv.) at $0{ }^{\circ} \mathrm{C}$ in an ice bath. After being stirred for $16 \mathrm{~h}$ at room temperature, saturated aqueous $\mathrm{NH}_{4} \mathrm{Cl}$ was added. The reaction mixture was extracted with EtOAc $\times 3$, washed with brine, dried over $\mathrm{Na}_{2} \mathrm{SO}_{4}$, and concentrated under reduced pressure. The resulting residue was passed through a short pad of silica to remove excessive imidazole ( $n$-hexane/EtOAc $=5 / 1)$ and azeotropically distilled with toluene to remove tert butyldimethylsilanol and give crude silylated product $(913.4 \mathrm{mg})$, which was used for the next step without further purification.

To a stirred solution of the silylated product in $\mathrm{MeOH}$ was added ${ }^{t} \mathrm{BuNH}_{2}$ ( $0.79 \mathrm{~mL}, 7.5 \mathrm{mmol}, 3$ equiv.), and the reaction mixture was stirred for $23 \mathrm{~h}$ at room temperature. The reaction mixture was cooled to $0{ }^{\circ} \mathrm{C}$ in an ice bath, $\mathrm{NaBH}_{4}$ (113.5 mg, $3 \mathrm{mmol}, 1.2$ equiv.) was then added, and stirring was continued for $1 \mathrm{~h}$ at $0{ }^{\circ} \mathrm{C}$. The reaction was quenched with the addition of water and concentrated under reduced pressure to remove most of the $\mathrm{MeOH}$. The aqueous solution was extracted with EtOAc $\times 3$, and the combined organic layers were washed with brine, dried over $\mathrm{Na}_{2} \mathrm{SO}_{4}$, and concentrated under reduced pressure to afford crude secondary amine, which was used for the next step without further purification.

1g was synthesized from silylated product under the same conditions as synthesis of $1 \mathrm{a}$. 
<smiles>N#CC(=O)N(CC(=O)c1ccccc1)Cc1ccc2c(Br)c(O)ccc2c1</smiles>

\section{$N$-((5-Bromo-6-hydroxynaphthalen-2-yl)methyl)- $N$-(tert-butyl)-2-}

\section{diazoacetamide (1g)}

$1 \mathrm{~g}$ was afforded as beige solid (69.8 mg, 3\% yield (5 steps)).: m.p. $112-114{ }^{\circ} \mathrm{C}$; $\mathrm{R}_{f}=0.7$ ( $n$-hexane/EtOAc, $\left.1 / 1\right) ;{ }^{1} \mathrm{H} \mathrm{NMR}\left(400 \mathrm{MHz}, \mathrm{CDCl}_{3}\right) \delta 8.04(\mathrm{~d}, J=8.4$ $\mathrm{Hz}, 1 \mathrm{H}), 7.72(\mathrm{~d}, J=8.4 \mathrm{~Hz}, 1 \mathrm{H}), 7.61(\mathrm{~s}, 1 \mathrm{H}), 7.41(\mathrm{~d}, J=8.4 \mathrm{~Hz}, 1 \mathrm{H}), 7.29(\mathrm{~d}$, $J=8.4 \mathrm{~Hz}, 1 \mathrm{H}), 4.78(\mathrm{~s}, 1 \mathrm{H}), 4.59(\mathrm{~s}, 2 \mathrm{H}), 1.51(\mathrm{~s}, 9 \mathrm{H}) ;{ }^{13} \mathrm{C} \mathrm{NMR}(100 \mathrm{MHz}$, $\left.\mathrm{CDCl}_{3}\right) \delta 168.1,150.8,134.8,131.7,129.8,129.2,127.8,126.4,125.8,118.0$, 106.1, 58.5, 49.0, 48.8, 29.1 (3C); IR (ATR) 3095, 2969, 2925, 2105, 1714, 1584, 1416, 1362, 1329, $1296 \mathrm{~cm}^{-1}$; HRMS (ESI-TOF) $[\mathrm{M}+\mathrm{Na}]^{+}$calcd for $\mathrm{C}_{17} \mathrm{H}_{18} \mathrm{BrN}_{3} \mathrm{NaO}_{2}{ }^{+} \mathrm{m} / z$ 398.0475, found $\mathrm{m} / z$ 398.0495.

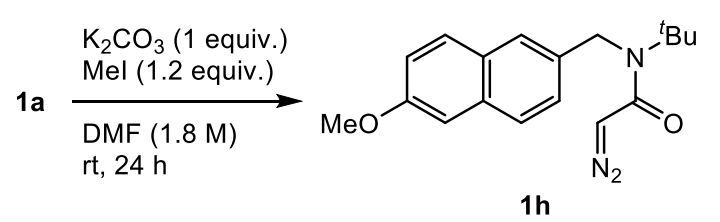

A $30 \mathrm{~mL}$ eggplant-shaped flask equipped with a magnetic stir bar was charged with $1 \mathrm{a}(61.5 \mathrm{mg}, 0.21 \mathrm{mmol})$ and $\mathrm{K}_{2} \mathrm{CO}_{3}(34.6 \mathrm{mg}, 0.25 \mathrm{mmol}, 1.2$ equiv.), which were subsequently dissolved in DMF (0.12 mL) under an argon gas atmosphere. To the stirred solution was added dropwise MeI $(15.6 \mu \mathrm{L}$, $0.25 \mathrm{mmol}, 1.2$ equiv.) at room temperature. After being stirred for $24 \mathrm{~h}$, the reaction mixture was quenched with the addition of water. The aqueous layer was extracted with $\mathrm{Et}_{2} \mathrm{O} \times 3$ and the combined organic layers were dried over $\mathrm{Na}_{2} \mathrm{SO}_{4}$, filtered through a plug of cotton, concentrated under reduced pressure, and purified by flash column chromatography ( $n$-hexane/EtOAc $=$ $6 / 1$ ) to afford $1 \mathrm{~h}$ as yellow oil (64.7 $\mathrm{mg}, 99 \%$ yield).

$N$-(tert-Butyl)-2-diazo- $N$-((6-methoxynaphthalen-2-yl)methyl)acetamide (1h) $\mathrm{R}_{f}=0.6$ ( $n$-hexane/EtOAc, 3/1); ${ }^{1} \mathrm{H} \mathrm{NMR}\left(400 \mathrm{MHz}, \mathrm{CDCl}_{3}\right) \delta 7.74(\mathrm{~d}, J=8.4$ $\mathrm{Hz}, 1 \mathrm{H}), 7.71(\mathrm{~d}, J=9.2 \mathrm{~Hz}, 1 \mathrm{H}), 7.59(\mathrm{~s}, 1 \mathrm{H}), 7.28(\mathrm{~d}, J=9.2 \mathrm{~Hz}, 1 \mathrm{H}), 7.18$ 
$(\mathrm{dd}, J=8.4,2.4 \mathrm{~Hz}, 1 \mathrm{H}), 7.14(\mathrm{~d}, J=2.4 \mathrm{~Hz}, 1 \mathrm{H}), 4.81(\mathrm{~s}, 1 \mathrm{H}), 4.56(\mathrm{~s}, 2 \mathrm{H})$, $3.93(\mathrm{~s}, 3 \mathrm{H}), 1.52(\mathrm{~s}, 9 \mathrm{H}) ;{ }^{13} \mathrm{C} \mathrm{NMR}\left(150 \mathrm{MHz}, \mathrm{CDCl}_{3}\right) \delta$ 168.2, 157.9, 134.1, 133.9, 129.3, 129.1, 127.7, 124.4, 124.3, 119.4, 105.9, 58.5, 55.5, 49.0, 49.0, 29.1 (3C); IR (ATR) 2963, 2101, 1739, 1607, 1484, 1400, 1360, 1264, 1210, 1194, $1171 \mathrm{~cm}^{-1}$; HRMS (ESI-TOF) $[\mathrm{M}+\mathrm{Na}]^{+}$calcd for $\mathrm{C}_{18} \mathrm{H}_{21} \mathrm{~N}_{3} \mathrm{NaO}_{2}{ }^{+} \mathrm{m} / Z$ 334.1526 , found $\mathrm{m} / \mathrm{z} 334.1541$.

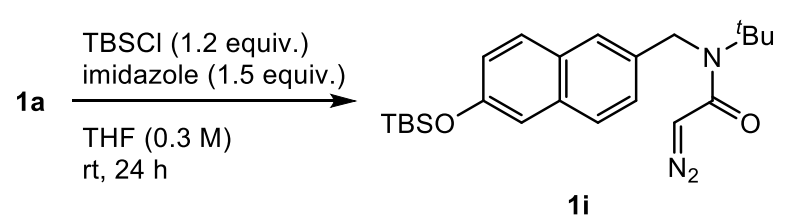

A $30 \mathrm{~mL}$ eggplant-shaped flask equipped with a magnetic stir bar was charged with 1a $(89.2 \mathrm{mg}, 0.3 \mathrm{mmol})$ and imidazole $(30.6 \mathrm{mg}, 0.45 \mathrm{mmol}$, 1.5 equiv.), which were subsequently dissolved in THF ( $1 \mathrm{~mL}$ ) under an argon gas atmosphere. To the stirred solution was added TBSCl $(54.3 \mathrm{mg}, 0.36$ mmol, 1.2 equiv.) at $0{ }^{\circ} \mathrm{C}$ in an ice bath. After being stirred for $24 \mathrm{~h}$ at room temperature, the reaction mixture was quenched with the addition of water. The aqueous layer was extracted with $\mathrm{Et}_{2} \mathrm{O} \times 3$ and the combined organic layers were dried over $\mathrm{Na}_{2} \mathrm{SO}_{4}$, filtered through a plug of cotton, concentrated under reduced pressure, and purified by flash column chromatography ( $n-$ hexane/EtOAc $=8 / 1)$ to afford $1 \mathrm{~g}$ as yellow oil $(99.4 \mathrm{mg}, 80 \%$ yield $)$.

\section{$N=$ (tert Butyl)- $N$-((6-((tert-butyldimethylsilyl)oxy)naphthalen-2-yl)methyl)- 2-diazoacetamide (1i)}

$\mathrm{R}_{f}=0.7$ ( $n$-hexane/EtOAc, 2/1); ${ }^{1} \mathrm{H}$ NMR (600 MHz, $\left.\mathrm{CDCl}_{3}\right) \delta 7.69(\mathrm{~d}, J=9.0$ $\mathrm{Hz}, 1 \mathrm{H}), 7.69(\mathrm{~d}, J=9.0 \mathrm{~Hz}, 1 \mathrm{H}), 7.59(\mathrm{~s}, 1 \mathrm{H}), 7.25(\mathrm{dd}, J=9.0,2.4 \mathrm{~Hz}, 1 \mathrm{H})$, $7.19(\mathrm{~d}, J=3.0 \mathrm{~Hz}, 1 \mathrm{H}), 7.10(\mathrm{dd}, J=9.0,3.0 \mathrm{~Hz}, 1 \mathrm{H}), 4.81(\mathrm{~s}, 1 \mathrm{H}), 4.55(\mathrm{~s}$, $2 \mathrm{H}), 1.52$ (s, 9H), $1.02(\mathrm{~s}, 9 \mathrm{H}), 0.25$ (s, 6H); $\left.{ }^{13} \mathrm{C} \mathrm{NMR} \mathrm{(150} \mathrm{MHz,} \mathrm{CDCl}_{3}\right) \delta$ 168.2, 153.8, 134.3, 133.9, 129.4, 129.2, 127.6, 124.2, 124.1, 122.9, 115.0, 58.5, 49.1, 49.0, 29.2 (3C), 25.9 (3C), 18.4, -4.2 (2C); IR (ATR) 2952, 2929, 2858, 2098, 1602, 1482, 1399, 1380, 1264, 1232, $1216 \mathrm{~cm}^{-1}$; HRMS (ESI-TOF) [M + Na]+ calcd for $\mathrm{C}_{23} \mathrm{H}_{33} \mathrm{~N}_{3} \mathrm{NaO}_{2} \mathrm{Si}^{+} \mathrm{m} / z$ 434.2234, found $\mathrm{m} / z 434.2241$. 


\section{Computational Details}

All the calculations were performed with Gaussian 16 program. ${ }^{[3]}$

\section{Mulliken Charge Distribution and Frontier Molecular Orbital of $\beta$-Naphthol}

The molecular structure optimizations were carried out using the hybrid density functional method based on $\omega$ B97XD functional and the cc-pVTZ basis set for $\mathrm{H}, \mathrm{C}$, and $\mathrm{O}$. The vibrational frequencies were computed at the same level to check whether each optimized structure is at an energy minimum on the potential energy surfaces (no imaginary frequency), and to evaluate its zero-point vibrational energy (ZPVE) and thermal corrections at $298.15 \mathrm{~K}$.

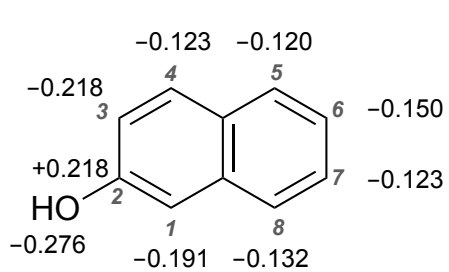

$0.191-0.132$

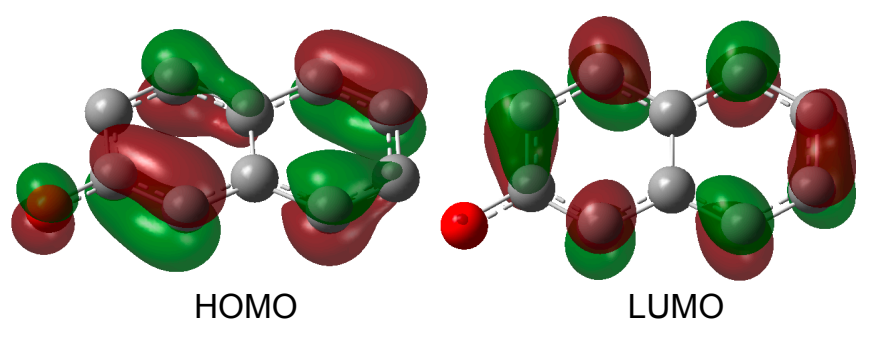

Energy (RwB97XD): -461.107554 A.U.

Zero-point correction=

(Hartree/Particle)

Thermal correction to Energy=

0.161079

Thermal correction to Enthalpy=

0.162023

Thermal correction to Gibbs Free Energy=

0.120520

Sum of electronic and zero-point Energies $=$ $-460.954430$

Sum of electronic and thermal Energies= $-460.946475$

Sum of electronic and thermal Enthalpies= $-460.945531$

Sum of electronic and thermal Free Energies= $-460.987033$

\section{Cartesian Coordinates}

$\begin{array}{llll}\text { Atom } & \mathrm{X} & \mathrm{Y} & \mathrm{Z}\end{array}$

$\begin{array}{lccc}\mathrm{C} & 1.852093 & 1.260232 & -0.000084 \\ \mathrm{C} & 2.678805 & -0.99614 & 0.000102\end{array}$




$\begin{array}{lrrc}\mathrm{C} & 0.522034 & 0.779055 & 0.000090 \\ \mathrm{H} & 2.018765 & 2.330236 & 0.000089 \\ \mathrm{C} & 1.407348 & -1.490583 & 0.000109 \\ \mathrm{H} & 3.521431 & -1.674438 & 0.000723 \\ \mathrm{C} & 0.290043 & -0.619732 & -0.000080 \\ \mathrm{C} & -0.592807 & 1.646779 & 0.000130 \\ \mathrm{H} & 1.234947 & -2.559283 & 0.000307 \\ \mathrm{C} & -1.034759 & -1.105582 & -0.000168 \\ \mathrm{C} & -1.865408 & 1.157536 & 0.000025 \\ \mathrm{H} & -0.425732 & 2.716379 & 0.000182 \\ \mathrm{C} & -2.090174 & -0.236866 & -0.000051 \\ \mathrm{H} & -1.223861 & -2.170492 & -0.000166 \\ \mathrm{H} & -2.711026 & 1.835595 & -0.000075 \\ \mathrm{O} & -3.349814 & -0.745755 & -0.000137 \\ \mathrm{H} & -3.984483 & -0.028991 & 0.001163 \\ \mathrm{C} & 2.907404 & 0.396008 & -0.000154 \\ \mathrm{H} & 3.921000 & 0.772798 & -0.000639\end{array}$

\section{Mechanistic Studies on Dearomative Spirocyclization of $\beta$-Naphthols}

The molecular structure optimizations were carried out using the hybrid density functional method based on Becke's three-parameter exchange function and the Lee-YangParr nonlocal correlation functional (B3LYP) ${ }^{[4]}$ and the LANL2DZ basis set for Ag, and the $6-31 \mathrm{G}^{*}$ basis set for $\mathrm{H}, \mathrm{C}, \mathrm{N}, \mathrm{O}, \mathrm{F}$ and $\mathrm{S}$. The vibrational frequencies were computed at the same level to check whether each optimized structure is at an energy minimum on the potential energy surfaces (no imaginary frequency) or a transition state (one imaginary frequency) and to evaluate its zero-point vibrational energy (ZPVE) and thermal corrections at $298.15 \mathrm{~K}$. The intrinsic reaction coordinate (IRC) method was used to track minimum energy paths from transition structures to the corresponding local minima. ${ }^{[5]}$ Single point energies were calculated at the RB3PW91 level using SDD basis set ${ }^{[6]}$ for $\mathrm{Ag}$ and $6-311++\mathrm{G}^{* *}$ basis set for $\mathrm{H}, \mathrm{C}, \mathrm{N}, \mathrm{O}, \mathrm{F}$ and $\mathrm{S}$ in $\mathrm{CH}_{2} \mathrm{Cl}_{2}$ solvent using $\mathrm{CPCM}$ model. 


\begin{tabular}{|c|c|c|c|}
\hline & $\begin{array}{c}\text { E(RB3LYP/6- } \\
\left.31 G^{*}\right)(\text { A.U.) }\end{array}$ & $\begin{array}{c}\text { Thermal } \\
\text { Correction to } \\
\text { Free Energy } \\
\text { (A.U.) }\end{array}$ & $\begin{array}{c}\text { Sum of Electronic } \\
\text { and Thermal Free } \\
\text { Energies } \\
\text { (A.U.) }\end{array}$ \\
\hline 1a+AgNTf 2 & -2946.817203 & 0.310667 & -1848962.316 \\
\hline TS1 & -2946.802981 & 0.311878 & -1848952.632 \\
\hline CP1 & -2946.809715 & 0.311874 & -1848956.86 \\
\hline TS2A & -2946.780869 & 0.309385 & -1848940.321 \\
\hline TS2B & -2946.779106 & 0.309294 & -1848939.272 \\
\hline TS2C & -2946.764332 & 0.312167 & -1848928.198 \\
\hline INT1 & -2946.829151 & 0.303944 & -1848974.033 \\
\hline CP2 & -2946.874147 & 0.304287 & -1849002.053 \\
\hline
\end{tabular}

\begin{tabular}{|c|c|c|c|}
\hline & $\begin{array}{c}\text { E(RB3PW91/ } \\
\left.6-311++G^{* *}\right) \\
(\text { A.U.) }\end{array}$ & $\begin{array}{c}\text { Thermal } \\
\text { Correction to } \\
\text { Free Energy } \\
\text { (A.U.) }\end{array}$ & $\begin{array}{c}\text { Sum of Electronic } \\
\text { and Thermal Free } \\
\text { Energies } \\
\text { (A.U.) }\end{array}$ \\
\hline 1a+AgNTf 2 & -2947.926064 & 0.310667 & -1849658.138 \\
\hline TS1 & -2947.91443 & 0.311878 & -1849650.077 \\
\hline CP1 & -2947.925639 & 0.311874 & -1849657.114 \\
\hline TS2A & -2947.890114 & 0.309385 & -1849636.383 \\
\hline TS2B & -2947.888362 & 0.309294 & -1849635.341 \\
\hline TS2C & -2947.872962 & 0.312167 & -1849623.874 \\
\hline INT1 & -2947.944093 & 0.303944 & -1849673.67 \\
\hline CP2 & -2947.985516 & 0.304287 & -1849699.448 \\
\hline
\end{tabular}




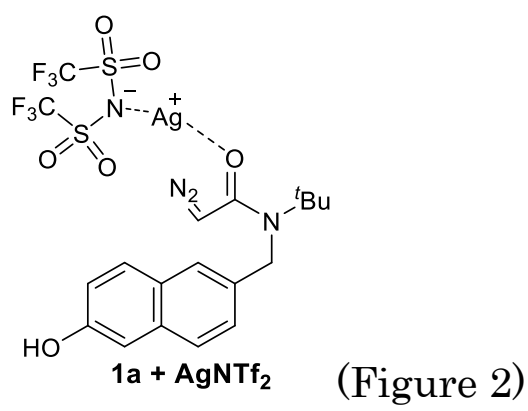

Zero-point correction $=$ 0.390951 (Hartree/Particle)

Thermal correction to Energy=

0.430293

Thermal correction to Enthalpy=

0.431237

Thermal correction to Gibbs Free Energy=

0.310667

Sum of electronic and zero-point Energies=

$-2946.426252$

Sum of electronic and thermal Energies=

$-2946.386910$

Sum of electronic and thermal Enthalpies= $-2946.385966$

Sum of electronic and thermal Free Energies= $-2946.506536$

\section{Cartesian Coordinates}

$\begin{array}{lccc}\text { Atom } & \mathrm{X} & \mathrm{Y} & \mathrm{Z} \\ \mathrm{Ag} & 1.49104 & -1.46036 & -0.18948 \\ \mathrm{C} & -1.48621 & -2.16885 & -2.06415 \\ \mathrm{H} & -2.33682 & -1.61238 & -2.42909 \\ \mathrm{C} & -1.26056 & -2.6282 & -0.69343 \\ \mathrm{O} & -0.09204 & -2.97063 & -0.33981 \\ \mathrm{~N} & -2.32359 & -2.68468 & 0.14594 \\ \mathrm{C} & -2.13828 & -3.03901 & 1.61876 \\ \mathrm{C} & -3.67166 & -2.47467 & -0.40926 \\ \mathrm{H} & -3.69404 & -2.92182 & -1.40985 \\ \mathrm{H} & -4.37478 & -3.06816 & 0.17487 \\ \mathrm{~N} & 0.39987 & -2.39546 & -3.60577 \\ \mathrm{~N} & -0.47955 & -2.28128 & -2.89649 \\ \mathrm{C} & -4.15307 & -1.03097 & -0.46931 \\ \mathrm{C} & -3.33363 & 0.0547 & -0.2389 \\ \mathrm{C} & -5.51744 & -0.80367 & -0.80928\end{array}$




\begin{tabular}{|c|c|c|c|}
\hline $\mathrm{C}$ & -3.8237 & 1.38561 & -0.33546 \\
\hline $\mathrm{H}$ & -2.28855 & -0.08141 & 0.02419 \\
\hline $\mathrm{C}$ & -6.02221 & 0.4704 & -0.90934 \\
\hline $\mathrm{H}$ & -6.16995 & -1.65614 & -0.98923 \\
\hline $\mathrm{C}$ & -5.19708 & 1.60786 & -0.67761 \\
\hline $\mathrm{H}$ & -7.06731 & 0.62447 & -1.16651 \\
\hline $\mathrm{C}$ & -5.68771 & 2.93164 & -0.77477 \\
\hline $\mathrm{H}$ & -6.72633 & 3.11632 & -1.03223 \\
\hline $\mathrm{C}$ & -4.84692 & 4.00329 & -0.54403 \\
\hline $\mathrm{C}$ & -2.98598 & 2.50936 & -0.10578 \\
\hline $\mathrm{C}$ & -3.48503 & 3.78744 & -0.20804 \\
\hline $\mathrm{H}$ & -2.8342 & 4.64231 & -0.03228 \\
\hline $\mathrm{H}$ & -1.94142 & 2.34832 & 0.14771 \\
\hline $\mathrm{O}$ & -5.36378 & 5.26252 & -0.65018 \\
\hline $\mathrm{H}$ & -4.66519 & 5.90968 & -0.46622 \\
\hline $\mathrm{N}$ & 2.56893 & 0.3732 & 0.18311 \\
\hline $\mathrm{S}$ & 4.20053 & 0.40551 & -0.12816 \\
\hline $\mathrm{S}$ & 1.6897 & 1.62512 & 0.80429 \\
\hline $\mathrm{O}$ & 4.93914 & 1.37145 & 0.67531 \\
\hline $\mathrm{O}$ & 4.60206 & -1.0008 & -0.2126 \\
\hline $\mathrm{O}$ & 2.22896 & 2.94953 & 0.5228 \\
\hline $\mathrm{O}$ & 0.28339 & 1.30252 & 0.51386 \\
\hline $\mathrm{C}$ & 1.84451 & 1.35738 & 2.65017 \\
\hline $\mathrm{C}$ & 4.24268 & 1.02203 & -1.89452 \\
\hline $\mathrm{F}$ & 3.69579 & 2.23183 & -1.98486 \\
\hline $\mathrm{F}$ & 5.51046 & 1.07095 & -2.30411 \\
\hline $\mathrm{F}$ & 3.5609 & 0.17222 & -2.67592 \\
\hline $\mathrm{F}$ & 1.3543 & 0.14716 & 2.96501 \\
\hline $\mathrm{F}$ & 3.11404 & 1.4268 & 3.0348 \\
\hline $\mathrm{F}$ & 1.13049 & 2.29092 & 3.28244 \\
\hline $\mathrm{C}$ & -3.46846 & -2.86325 & 2.37811 \\
\hline $\mathrm{H}$ & -4.21872 & -3.61105 & 2.10256 \\
\hline $\mathrm{H}$ & -3.26469 & -3.00005 & 3.44396 \\
\hline
\end{tabular}




$\begin{array}{llll}\mathrm{H} & -3.89315 & -1.86377 & 2.24484 \\ \mathrm{C} & -1.10849 & -2.09664 & 2.27511 \\ \mathrm{H} & -0.09872 & -2.27029 & 1.9041 \\ \mathrm{H} & -1.36803 & -1.04563 & 2.1153 \\ \mathrm{H} & -1.09693 & -2.2799 & 3.35455 \\ \mathrm{C} & -1.69324 & -4.50975 & 1.72707 \\ \mathrm{H} & -1.59412 & -4.78681 & 2.78265 \\ \mathrm{H} & -2.43756 & -5.17402 & 1.27297 \\ \mathrm{H} & -0.73259 & -4.66933 & 1.23442\end{array}$

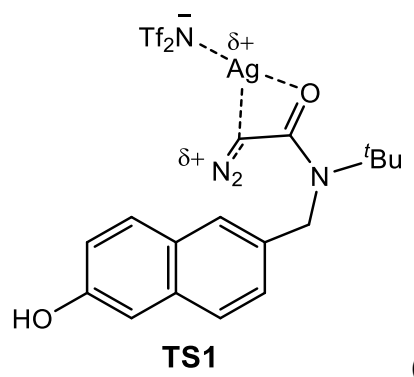

(Figure 2)

Zero-point correction= 0.390225 (Hartree/Particle)

Thermal correction to Energy= 0.428914

Thermal correction to Enthalpy=

0.429858

Thermal correction to Gibbs Free Energy= 0.311878

Sum of electronic and zero-point Energies= $-2946.412756$

Sum of electronic and thermal Energies= $-2946.374067$

Sum of electronic and thermal Enthalpies= $-2946.373122$

Sum of electronic and thermal Free Energies= $-2946.491102$

\section{Cartesian Coordinates}

\begin{tabular}{lccc} 
Atom & $\mathrm{X}$ & $\mathrm{Y}$ & $\mathrm{Z}$ \\
\hdashline $\mathrm{Ag}$ & 0.87109 & -1.1831 & -0.96533 \\
$\mathrm{C}$ & -1.3719 & -1.63938 & -2.09569 \\
$\mathrm{H}$ & -1.92279 & -0.71592 & -2.23308 \\
$\mathrm{C}$ & -1.49866 & -2.55054 & -0.89968
\end{tabular}


O

$\mathrm{N}$

C

C

$\mathrm{H}$

$\mathrm{H}$

$\mathrm{N}$

$\mathrm{N}$

C

C

C

C

$\mathrm{H}$

C

$\mathrm{H}$

C

$\mathrm{H}$

C

$\mathrm{H}$

C

C

C

$\mathrm{H}$

$\mathrm{H}$

$\mathrm{O}$

$\mathrm{H}$

N

S

S

O

O

O

O

$$
\begin{array}{lcl}
-0.55046 & -3.32707 & -0.67078 \\
-2.59754 & -2.40805 & -0.1201 \\
-2.6568 & -3.10594 & 1.23695 \\
-3.7849 & -1.72816 & -0.6695 \\
-3.83984 & -1.95574 & -1.74008 \\
-4.66813 & -2.20123 & -0.23682 \\
-0.57946 & -2.70138 & -4.16749 \\
-0.95573 & -2.20993 & -3.21921 \\
-3.86151 & -0.22142 & -0.46693 \\
-2.88764 & 0.5151 & 0.17476 \\
-5.00432 & 0.45133 & -0.98764 \\
-3.00613 & 1.92482 & 0.32608 \\
-2.00607 & 0.03142 & 0.58653 \\
-5.14828 & 1.81126 & -0.85782 \\
-5.77614 & -0.12534 & -1.49434 \\
-4.15821 & 2.59539 & -0.19909 \\
-6.02796 & 2.30774 & -1.26005 \\
-4.27904 & 3.99744 & -0.05226 \\
-5.14637 & 4.51935 & -0.44526 \\
-3.2918 & 4.71647 & 0.59383 \\
-2.01053 & 2.69347 & 0.98569 \\
-2.14964 & 4.05623 & 1.11629 \\
-1.37999 & 4.63595 & 1.62304 \\
-1.13497 & 2.19107 & 1.38747 \\
-3.45067 & 6.06713 & 0.7113 \\
-2.68623 & 6.43799 & 1.17911 \\
2.27953 & 0.14215 & 0.05343 \\
3.6623 & 0.46808 & -0.7984 \\
1.9849 & 0.57253 & 1.61704 \\
4.85565 & 0.64095 & 0.02035 \\
3.63009 & -0.46826 & -1.92913 \\
2.70544 & 1.75742 & 2.06677 \\
0.52833 & 0.46073 & 1.79183
\end{array}
$$




$\begin{array}{llll}\text { C } & 2.66923 & -0.88117 & 2.57708 \\ \mathrm{C} & 3.2766 & 2.136 & -1.55409 \\ \mathrm{~F} & 3.05256 & 3.04251 & -0.60603 \\ \mathrm{~F} & 4.30988 & 2.51829 & -2.30505 \\ \mathrm{~F} & 2.18699 & 2.03041 & -2.32776 \\ \mathrm{~F} & 2.04535 & -2.00377 & 2.18377 \\ \mathrm{~F} & 3.97465 & -1.01147 & 2.36592 \\ \mathrm{~F} & 2.43953 & -0.68968 & 3.87679 \\ \mathrm{C} & -3.87908 & -2.60913 & 2.03322 \\ \mathrm{H} & -4.83017 & -2.96789 & 1.62698 \\ \mathrm{H} & -3.80062 & -3.00635 & 3.04941 \\ \mathrm{H} & -3.91066 & -1.51785 & 2.10279 \\ \mathrm{C} & -1.39565 & -2.76851 & 2.05811 \\ \mathrm{H} & -0.48862 & -3.15385 & 1.59477 \\ \mathrm{H} & -1.28712 & -1.68837 & 2.19981 \\ \mathrm{H} & -1.49106 & -3.22783 & 3.04771 \\ \mathrm{C} & -2.77976 & -4.6237 & 1.00832 \\ \mathrm{H} & -2.86699 & -5.13622 & 1.97292 \\ \mathrm{H} & -3.67598 & -4.8581 & 0.42151 \\ \mathrm{H} & -1.90372 & -5.01362 & 0.48639\end{array}$

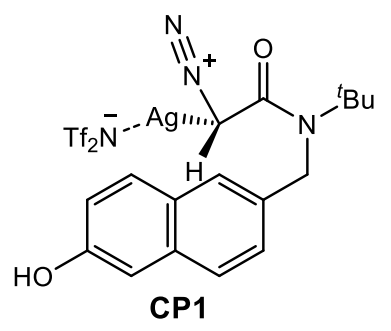

(Figure 2)

Zero-point correction $=$ 0.390225 (Hartree/Particle)

Thermal correction to Energy= 0.428914

Thermal correction to Enthalpy= 0.429858

Thermal correction to Gibbs Free Energy= 0.311878

Sum of electronic and zero-point Energies= $-2946.412756$

Sum of electronic and thermal Energies= $-2946.374067$ 
Sum of electronic and thermal Enthalpies= $-2946.373122$

Sum of electronic and thermal Free Energies=

Cartesian Coordinates

\begin{tabular}{|c|c|c|c|}
\hline Atom & $\mathrm{X}$ & $\mathrm{Y}$ & $\mathrm{Z}$ \\
\hline $\mathrm{Ag}$ & -0.90295 & -0.21523 & 1.07009 \\
\hline $\mathrm{C}$ & 1.05377 & -0.94454 & 1.85897 \\
\hline $\mathrm{H}$ & 1.72566 & -0.16636 & 1.48874 \\
\hline $\mathrm{C}$ & 1.27523 & -2.40101 & 1.40964 \\
\hline $\mathrm{O}$ & 1.04409 & -3.29049 & 2.22285 \\
\hline $\mathrm{N}$ & 1.71601 & -2.57576 & 0.12843 \\
\hline $\mathrm{C}$ & 1.88021 & -4.00523 & -0.37949 \\
\hline $\mathrm{C}$ & 1.79594 & -1.41841 & -0.78752 \\
\hline $\mathrm{H}$ & 1.88527 & -1.81098 & -1.7975 \\
\hline $\mathrm{H}$ & 0.84508 & -0.87296 & -0.78936 \\
\hline $\mathrm{N}$ & 0.96341 & -0.91219 & 4.33792 \\
\hline $\mathrm{N}$ & 1.02077 & -0.90234 & 3.21843 \\
\hline $\mathrm{C}$ & 2.93593 & -0.44208 & -0.53737 \\
\hline $\mathrm{C}$ & 2.74282 & 0.90229 & -0.80565 \\
\hline $\mathrm{C}$ & 4.21257 & -0.8796 & -0.09398 \\
\hline $\mathrm{C}$ & 3.793 & 1.84415 & -0.65685 \\
\hline $\mathrm{H}$ & 1.77113 & 1.25116 & -1.15263 \\
\hline $\mathrm{C}$ & 5.24998 & 0.0121 & 0.06395 \\
\hline $\mathrm{H}$ & 4.36584 & -1.931 & 0.13217 \\
\hline $\mathrm{C}$ & 5.07901 & 1.39601 & -0.21189 \\
\hline $\mathrm{H}$ & 6.22109 & -0.33885 & 0.40525 \\
\hline $\mathrm{C}$ & 6.12839 & 2.33858 & -0.05719 \\
\hline $\mathrm{H}$ & 7.1049 & 1.99547 & 0.28124 \\
\hline $\mathrm{C}$ & 5.91476 & 3.6738 & -0.33467 \\
\hline $\mathrm{C}$ & 3.61226 & 3.22901 & -0.9311 \\
\hline $\mathrm{C}$ & 4.64174 & 4.12368 & -0.77656 \\
\hline $\mathrm{H}$ & 4.5136 & 5.1808 & -0.98597 \\
\hline
\end{tabular}


Supporting Information

\begin{tabular}{|c|c|c|c|}
\hline $\mathrm{H}$ & 2.63918 & 3.57302 & -1.27222 \\
\hline $\mathrm{O}$ & 6.87664 & 4.6327 & -0.20977 \\
\hline $\mathrm{H}$ & 7.70218 & 4.21947 & 0.08923 \\
\hline $\mathrm{N}$ & -2.5336 & 0.53423 & -0.13531 \\
\hline $\mathrm{S}$ & -2.24376 & 0.73353 & -1.74853 \\
\hline $\mathrm{S}$ & -4.01698 & 0.64243 & 0.60239 \\
\hline $\mathrm{O}$ & -3.21091 & 1.57575 & -2.43869 \\
\hline $\mathrm{O}$ & -0.79859 & 0.97118 & -1.86596 \\
\hline $\mathrm{O}$ & -5.14698 & 0.3573 & -0.27248 \\
\hline $\mathrm{O}$ & -3.84292 & -0.04068 & 1.88906 \\
\hline $\mathrm{C}$ & -4.10436 & 2.46211 & 1.03044 \\
\hline $\mathrm{C}$ & -2.46617 & -1.00437 & -2.41056 \\
\hline $\mathrm{F}$ & -3.69565 & -1.45011 & -2.18254 \\
\hline $\mathrm{F}$ & -2.21803 & -1.01034 & -3.71997 \\
\hline $\mathrm{F}$ & -1.58406 & -1.82453 & -1.80039 \\
\hline $\mathrm{F}$ & -3.08431 & 2.77439 & 1.84114 \\
\hline $\mathrm{F}$ & -4.03342 & 3.20663 & -0.06948 \\
\hline $\mathrm{F}$ & -5.25411 & 2.70005 & 1.66167 \\
\hline $\mathrm{C}$ & 2.43562 & -4.01813 & -1.81533 \\
\hline $\mathrm{H}$ & 3.37088 & -3.45537 & -1.90912 \\
\hline $\mathrm{H}$ & 2.65248 & -5.05834 & -2.07686 \\
\hline $\mathrm{H}$ & 1.71924 & -3.6483 & -2.55613 \\
\hline $\mathrm{C}$ & 0.50442 & -4.70101 & -0.37967 \\
\hline $\mathrm{H}$ & 0.10032 & -4.78008 & 0.63078 \\
\hline $\mathrm{H}$ & -0.20548 & -4.14591 & -1.00379 \\
\hline $\mathrm{H}$ & 0.6007 & -5.71113 & -0.79345 \\
\hline $\mathrm{C}$ & 2.89004 & -4.75929 & 0.51102 \\
\hline $\mathrm{H}$ & 2.99906 & -5.78428 & 0.1401 \\
\hline $\mathrm{H}$ & 3.87637 & -4.28248 & 0.46768 \\
\hline $\mathrm{H}$ & 2.56123 & -4.80034 & 1.54828 \\
\hline
\end{tabular}




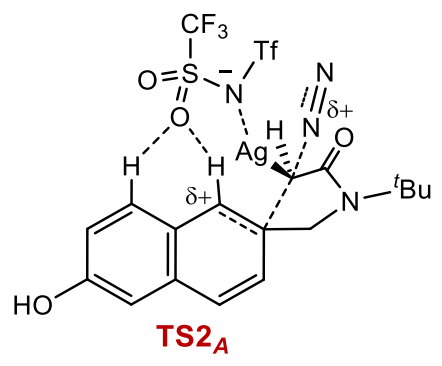

(Figure 2)

Zero-point correction $=$

Thermal correction to Energy=

Thermal correction to Enthalpy=

Thermal correction to Gibbs Free Energy=

Sum of electronic and zero-point Energies=

Sum of electronic and thermal Energies=

Sum of electronic and thermal Enthalpies=

Sum of electronic and thermal Free Energies=
0.388381 (Hartree/Particle)

0.427673

0.428618

0.309385

$-2946.392488$

$-2946.353195$

$-2946.352251$

$-2946.471484$

\section{Cartesian Coordinates}

$\begin{array}{lccc}\text { Atom } & \mathrm{X} & \mathrm{Y} & \mathrm{Z} \\ \mathrm{Ag} & -0.30376 & -0.42018 & 0.72663 \\ \mathrm{C} & 1.69885 & -0.59646 & 1.4147 \\ \mathrm{H} & 2.24657 & 0.31133 & 1.6559 \\ \mathrm{C} & 2.53344 & -1.85482 & 1.2085 \\ \mathrm{O} & 2.64728 & -2.65166 & 2.14204 \\ \mathrm{~N} & 3.06145 & -2.0547 & -0.04105 \\ \mathrm{C} & 3.70056 & -3.40497 & -0.34215 \\ \mathrm{C} & 2.71984 & -1.11631 & -1.12892 \\ \mathrm{H} & 3.3826 & -1.3315 & -1.96454 \\ \mathrm{H} & 1.69438 & -1.28662 & -1.48612 \\ \mathrm{~N} & 0.91598 & -0.9946 & 4.31753 \\ \mathrm{~N} & 1.36259 & -0.89019 & 3.31116 \\ \mathrm{C} & 2.89047 & 0.34139 & -0.75415 \\ \mathrm{C} & 1.9051 & 1.26398 & -1.0763 \\ \mathrm{C} & 4.10832 & 0.80771 & -0.17683\end{array}$


Supporting Information

\begin{tabular}{|c|c|c|c|}
\hline $\mathrm{C}$ & 2.07015 & 2.64588 & -0.81178 \\
\hline $\mathrm{H}$ & 0.98233 & 0.93964 & -1.55095 \\
\hline $\mathrm{C}$ & 4.29588 & 2.14279 & 0.0904 \\
\hline $\mathrm{H}$ & 4.88808 & 0.09002 & 0.0648 \\
\hline $\mathrm{C}$ & 3.28816 & 3.10496 & -0.21317 \\
\hline $\mathrm{H}$ & 5.22818 & 2.48347 & 0.53454 \\
\hline $\mathrm{C}$ & 3.45012 & 4.48575 & 0.05273 \\
\hline $\mathrm{H}$ & 4.37567 & 4.83734 & 0.50586 \\
\hline $\mathrm{C}$ & 2.44251 & 5.37954 & -0.26293 \\
\hline $\mathrm{C}$ & 1.05292 & 3.59349 & -1.1199 \\
\hline $\mathrm{C}$ & 1.23194 & 4.9283 & -0.85298 \\
\hline $\mathrm{H}$ & 0.46588 & 5.66203 & -1.08162 \\
\hline $\mathrm{H}$ & 0.12818 & 3.23866 & -1.56686 \\
\hline $\mathrm{O}$ & 2.53308 & 6.71866 & -0.03651 \\
\hline $\mathrm{H}$ & 3.39405 & 6.92009 & 0.36372 \\
\hline $\mathrm{N}$ & -2.28691 & -0.1821 & -0.11768 \\
\hline $\mathrm{S}$ & -2.46474 & 0.18891 & -1.71641 \\
\hline $\mathrm{S}$ & -3.52679 & -0.64453 & 0.88653 \\
\hline $\mathrm{O}$ & -3.8024 & 0.62717 & -2.0905 \\
\hline $\mathrm{O}$ & -1.27858 & 0.97023 & -2.09111 \\
\hline $\mathrm{O}$ & -4.65901 & -1.26211 & 0.20807 \\
\hline $\mathrm{O}$ & -2.86601 & -1.2928 & 2.02535 \\
\hline $\mathrm{C}$ & -4.12086 & 1.00083 & 1.55188 \\
\hline $\mathrm{C}$ & -2.18656 & -1.46906 & -2.53933 \\
\hline $\mathrm{F}$ & -3.08561 & -2.35869 & -2.13259 \\
\hline $\mathrm{F}$ & -2.26365 & -1.3215 & -3.86178 \\
\hline $\mathrm{F}$ & -0.95494 & -1.92032 & -2.22573 \\
\hline $\mathrm{F}$ & -3.11757 & 1.60176 & 2.20539 \\
\hline $\mathrm{F}$ & -4.53194 & 1.78863 & 0.56172 \\
\hline $\mathrm{F}$ & -5.1268 & 0.78293 & 2.39967 \\
\hline $\mathrm{C}$ & 4.21376 & -3.45825 & -1.79256 \\
\hline $\mathrm{H}$ & 4.97361 & -2.69606 & -1.99896 \\
\hline $\mathrm{H}$ & 4.68825 & -4.43356 & -1.9387 \\
\hline
\end{tabular}




$\begin{array}{llll}\mathrm{H} & 3.41233 & -3.37635 & -2.53394 \\ \mathrm{C} & 2.65314 & -4.52275 & -0.16241 \\ \mathrm{H} & 2.28674 & -4.56327 & 0.86381 \\ \mathrm{H} & 1.80326 & -4.36744 & -0.83746 \\ \mathrm{H} & 3.10295 & -5.49144 & -0.40726 \\ \mathrm{C} & 4.91493 & -3.6152 & 0.58584 \\ \mathrm{H} & 5.39863 & -4.56694 & 0.33907 \\ \mathrm{H} & 5.6511 & -2.81603 & 0.43887 \\ \mathrm{H} & 4.62041 & -3.6359 & 1.634\end{array}$

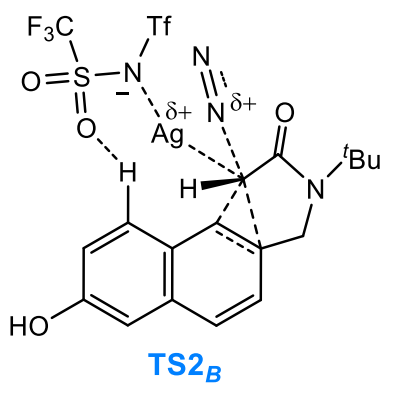

(Figure 2)

Zero-point correction= 0.388891 (Hartree/Particle)

Thermal correction to Energy= 0.427726

Thermal correction to Enthalpy=

0.428670

Thermal correction to Gibbs Free Energy=

0.312167

Sum of electronic and zero-point Energies= $-2946.375441$

Sum of electronic and thermal Energies= $-2946.336606$

Sum of electronic and thermal Enthalpies= $-2946.335662$

Sum of electronic and thermal Free Energies= $-2946.452165$

\section{Cartesian Coordinates}

$\begin{array}{lccc}\text { Atom } & \mathrm{X} & \mathrm{Y} & \mathrm{Z} \\ \mathrm{Ag} & 0.24449 & -0.64637 & 0.85368 \\ \mathrm{C} & 2.22494 & -0.27896 & 1.50736 \\ \mathrm{H} & 2.43947 & 0.56977 & 2.15521 \\ \mathrm{C} & 3.36904 & -0.9192 & 0.74828\end{array}$


Supporting Information

\begin{tabular}{|c|c|c|c|}
\hline $\mathrm{O}$ & 3.50577 & -2.14003 & 0.8119 \\
\hline $\mathrm{N}$ & 4.10735 & -0.07925 & -0.04679 \\
\hline $\mathrm{C}$ & 5.05085 & -0.67532 & -1.07717 \\
\hline $\mathrm{C}$ & 4.15527 & 1.34697 & 0.32136 \\
\hline $\mathrm{H}$ & 4.50345 & 1.44982 & 1.35943 \\
\hline $\mathrm{H}$ & 4.92086 & 1.82277 & -0.28935 \\
\hline $\mathrm{N}$ & 2.06093 & -2.20953 & 3.86389 \\
\hline $\mathrm{N}$ & 2.29882 & -1.48157 & 3.06604 \\
\hline $\mathrm{C}$ & 2.84421 & 2.09582 & 0.15734 \\
\hline $\mathrm{C}$ & 1.89699 & 1.74154 & -0.79058 \\
\hline $\mathrm{C}$ & 2.61431 & 3.24956 & 0.96079 \\
\hline $\mathrm{C}$ & 0.70505 & 2.49479 & -0.96237 \\
\hline $\mathrm{H}$ & 2.05709 & 0.87222 & -1.42285 \\
\hline $\mathrm{C}$ & 1.47419 & 4.00166 & 0.81314 \\
\hline $\mathrm{H}$ & 3.35735 & 3.53636 & 1.70231 \\
\hline $\mathrm{C}$ & 0.47828 & 3.65053 & -0.14435 \\
\hline $\mathrm{H}$ & 1.31266 & 4.87578 & 1.43895 \\
\hline $\mathrm{C}$ & -0.7182 & 4.38701 & -0.29256 \\
\hline $\mathrm{H}$ & -0.91022 & 5.25723 & 0.32757 \\
\hline $\mathrm{C}$ & -1.67148 & 3.98755 & -1.21187 \\
\hline $\mathrm{C}$ & -0.28553 & 2.12602 & -1.91089 \\
\hline $\mathrm{C}$ & -1.4466 & 2.85219 & -2.03248 \\
\hline $\mathrm{H}$ & -2.20935 & 2.54226 & -2.74361 \\
\hline $\mathrm{H}$ & -0.13711 & 1.23789 & -2.51655 \\
\hline $\mathrm{O}$ & -2.82016 & 4.71241 & -1.30065 \\
\hline $\mathrm{H}$ & -3.42219 & 4.28414 & -1.92966 \\
\hline $\mathrm{N}$ & -1.77156 & -0.98033 & 0.14748 \\
\hline $\mathrm{S}$ & -3.00273 & -0.71111 & 1.22479 \\
\hline $\mathrm{S}$ & -1.9788 & -1.41356 & -1.43533 \\
\hline $\mathrm{O}$ & -4.24868 & -1.38973 & 0.89045 \\
\hline $\mathrm{O}$ & -2.39386 & -0.82543 & 2.55282 \\
\hline $\mathrm{O}$ & -3.2189 & -0.93202 & -2.03568 \\
\hline $\mathrm{O}$ & -0.68277 & -1.16721 & -2.0829 \\
\hline
\end{tabular}




\begin{tabular}{|c|c|c|c|}
\hline $\mathrm{C}$ & -2.10054 & -3.28006 & -1.3464 \\
\hline $\mathrm{C}$ & -3.32444 & 1.12223 & 1.02188 \\
\hline $\mathrm{F}$ & -3.70791 & 1.40619 & -0.22437 \\
\hline $\mathrm{F}$ & -4.28423 & 1.48773 & 1.87298 \\
\hline $\mathrm{F}$ & -2.20698 & 1.8071 & 1.302 \\
\hline $\mathrm{F}$ & -0.98374 & -3.76845 & -0.79347 \\
\hline $\mathrm{F}$ & -3.14981 & -3.65309 & -0.62042 \\
\hline $\mathrm{F}$ & -2.22074 & -3.75766 & -2.58693 \\
\hline $\mathrm{C}$ & 5.59687 & 0.42572 & -2.00509 \\
\hline $\mathrm{H}$ & 6.31816 & 1.08601 & -1.51186 \\
\hline $\mathrm{H}$ & 6.12723 & -0.05983 & -2.82981 \\
\hline $\mathrm{H}$ & 4.79577 & 1.03316 & -2.44091 \\
\hline $\mathrm{C}$ & 4.27439 & -1.67624 & -1.95863 \\
\hline $\mathrm{H}$ & 3.8703 & -2.50415 & -1.37755 \\
\hline $\mathrm{H}$ & 3.45073 & -1.17547 & -2.48016 \\
\hline $\mathrm{H}$ & 4.9526 & -2.08469 & -2.71535 \\
\hline $\mathrm{C}$ & 6.22755 & -1.36434 & -0.35915 \\
\hline $\mathrm{H}$ & 6.92965 & -1.76827 & -1.0975 \\
\hline $\mathrm{H}$ & 6.77395 & -0.64799 & 0.2662 \\
\hline $\mathrm{H}$ & 5.87261 & -2.18297 & 0.26914 \\
\hline
\end{tabular}

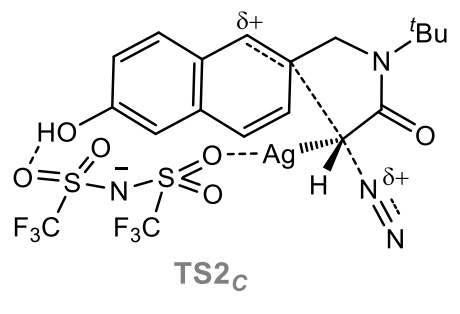

Zero-point correction $=$

Thermal correction to Energy=

Thermal correction to Enthalpy=

Thermal correction to Gibbs Free Energy=

Sum of electronic and zero-point Energies=

Sum of electronic and thermal Energies=
0.387681 (Hartree/Particle)
0.427160
0.428104
0.309294
$-2946.391425$
$-2946.351946$ 
Sum of electronic and thermal Enthalpies= $-2946.351002$

Sum of electronic and thermal Free Energies=

Cartesian Coordinates

\begin{tabular}{|c|c|c|c|}
\hline Atom & $\mathrm{X}$ & $\mathrm{Y}$ & $\mathrm{Z}$ \\
\hline $\mathrm{Ag}$ & 0.86997 & -0.8617 & -0.21971 \\
\hline $\mathrm{C}$ & 2.77702 & -0.41864 & 0.65883 \\
\hline $\mathrm{H}$ & 2.76124 & 0.30644 & 1.46751 \\
\hline $\mathrm{C}$ & 4.12937 & -0.77904 & 0.05433 \\
\hline $\mathrm{O}$ & 4.43708 & -1.97446 & 0.07229 \\
\hline $\mathrm{N}$ & 4.94333 & 0.19849 & -0.45818 \\
\hline $\mathrm{C}$ & 6.26284 & -0.24551 & -1.08787 \\
\hline $\mathrm{C}$ & 4.47416 & 1.5659 & -0.8011 \\
\hline $\mathrm{H}$ & 5.28893 & 2.25447 & -0.57273 \\
\hline $\mathrm{H}$ & 4.3094 & 1.61049 & -1.88563 \\
\hline $\mathrm{N}$ & 2.52431 & -2.65175 & 2.60972 \\
\hline $\mathrm{N}$ & 2.77308 & -1.8331 & 1.90734 \\
\hline $\mathrm{C}$ & 3.23384 & 2.05513 & -0.10153 \\
\hline $\mathrm{C}$ & 2.01723 & 2.07473 & -0.76923 \\
\hline $\mathrm{C}$ & 3.29582 & 2.59858 & 1.21826 \\
\hline $\mathrm{C}$ & 0.82779 & 2.51511 & -0.1432 \\
\hline $\mathrm{H}$ & 1.96498 & 1.72978 & -1.80092 \\
\hline $\mathrm{C}$ & 2.15677 & 3.02595 & 1.85776 \\
\hline $\mathrm{H}$ & 4.25678 & 2.64616 & 1.72574 \\
\hline $\mathrm{C}$ & 0.87814 & 2.95865 & 1.22249 \\
\hline $\mathrm{H}$ & 2.21389 & 3.41469 & 2.87145 \\
\hline $\mathrm{C}$ & -0.32311 & 3.26349 & 1.8958 \\
\hline $\mathrm{H}$ & -0.30994 & 3.55945 & 2.94045 \\
\hline $\mathrm{C}$ & -1.54615 & 3.10821 & 1.25547 \\
\hline $\mathrm{C}$ & -0.43046 & 2.46703 & -0.79998 \\
\hline $\mathrm{C}$ & -1.5875 & 2.75649 & -0.12384 \\
\hline $\mathrm{H}$ & -2.54733 & 2.6795 & -0.61944 \\
\hline
\end{tabular}


Supporting Information

\begin{tabular}{|c|c|c|c|}
\hline $\mathrm{H}$ & -0.47373 & 2.16288 & -1.84293 \\
\hline $\mathrm{O}$ & -2.67863 & 3.30271 & 1.95721 \\
\hline $\mathrm{H}$ & -3.41533 & 2.82188 & 1.52041 \\
\hline $\mathrm{N}$ & -2.91194 & -0.18493 & -0.13607 \\
\hline $\mathrm{S}$ & -4.4849 & 0.20919 & 0.07929 \\
\hline $\mathrm{S}$ & -2.41336 & -1.67083 & -0.45426 \\
\hline $\mathrm{O}$ & -5.35447 & -0.86133 & 0.5575 \\
\hline $\mathrm{O}$ & -4.50144 & 1.51665 & 0.75834 \\
\hline $\mathrm{O}$ & -3.30875 & -2.59387 & -1.14073 \\
\hline $\mathrm{O}$ & -1.03441 & -1.54312 & -1.03857 \\
\hline $\mathrm{C}$ & -2.07462 & -2.43545 & 1.2234 \\
\hline $\mathrm{C}$ & -5.05023 & 0.61968 & -1.65662 \\
\hline $\mathrm{F}$ & -4.88532 & -0.42697 & -2.4633 \\
\hline $\mathrm{F}$ & -6.33926 & 0.95941 & -1.62343 \\
\hline $\mathrm{F}$ & -4.3397 & 1.65324 & -2.13361 \\
\hline $\mathrm{F}$ & -1.13456 & -1.71484 & 1.87397 \\
\hline $\mathrm{F}$ & -3.17898 & -2.4603 & 1.95695 \\
\hline $\mathrm{F}$ & -1.61042 & -3.67607 & 1.05801 \\
\hline $\mathrm{C}$ & 7.09038 & 0.97658 & -1.5377 \\
\hline $\mathrm{H}$ & 7.3485 & 1.63581 & -0.70173 \\
\hline $\mathrm{H}$ & 8.02952 & 0.60095 & -1.95491 \\
\hline $\mathrm{H}$ & 6.6054 & 1.56702 & -2.32015 \\
\hline $\mathrm{C}$ & 5.97275 & -1.11982 & -2.32393 \\
\hline $\mathrm{H}$ & 5.42989 & -2.02484 & -2.04895 \\
\hline $\mathrm{H}$ & 5.38503 & -0.56348 & -3.06441 \\
\hline $\mathrm{H}$ & 6.91571 & -1.41158 & -2.79964 \\
\hline $\mathrm{C}$ & 7.11528 & -0.99788 & -0.04445 \\
\hline $\mathrm{H}$ & 8.09511 & -1.22264 & -0.48006 \\
\hline $\mathrm{H}$ & 7.27723 & -0.36886 & 0.83861 \\
\hline $\mathrm{H}$ & 6.65027 & -1.93083 & 0.26699 \\
\hline
\end{tabular}


int

(Figure 2)

Zero-point correction $=$

Thermal correction to Energy=

Thermal correction to Enthalpy=

Thermal correction to Gibbs Free Energy=

Sum of electronic and zero-point Energies=

Sum of electronic and thermal Energies=

Sum of electronic and thermal Enthalpies=

Sum of electronic and thermal Free Energies=
0.388117 (Hartree/Particle)

$$
0.428748
$$

0.429692

0.303944

$-2946.441034$

$-2946.400403$

$-2946.399459$

$-2946.525207$

\section{Cartesian Coordinates}

$\begin{array}{lccc}\text { Atom } & \mathrm{X} & \mathrm{Y} & \mathrm{Z} \\ \mathrm{Ag} & -0.18357 & -0.36575 & 0.66453 \\ \mathrm{C} & 1.95797 & -0.52876 & 1.04082 \\ \mathrm{H} & 2.12329 & -0.00011 & 1.97841 \\ \mathrm{C} & 2.2978 & -1.98607 & 1.08987 \\ \mathrm{O} & 2.02349 & -2.72908 & 2.02572 \\ \mathrm{~N} & 2.98456 & -2.34597 & -0.06365 \\ \mathrm{C} & 3.1343 & -3.77465 & -0.48418 \\ \mathrm{C} & 2.97436 & -1.25001 & -1.01885 \\ \mathrm{H} & 3.87284 & -1.24055 & -1.63941 \\ \mathrm{H} & 2.09613 & -1.28083 & -1.68307 \\ \mathrm{~N} & -0.77907 & -2.30992 & 4.25465 \\ \mathrm{~N} & -0.51511 & -1.30219 & 3.88681 \\ \mathrm{C} & 2.89704 & 0.02371 & -0.12849 \\ \mathrm{C} & 2.06585 & 1.10541 & -0.6535 \\ \mathrm{C} & 4.20479 & 0.46631 & 0.42556 \\ \mathrm{C} & 2.33366 & 2.45291 & -0.40014 \\ \mathrm{H} & 1.19239 & 0.85124 & -1.24731\end{array}$


Supporting Information

\begin{tabular}{|c|c|c|c|}
\hline $\mathrm{C}$ & 4.50221 & 1.76847 & 0.6197 \\
\hline $\mathrm{H}$ & 4.89488 & -0.31504 & 0.73274 \\
\hline $\mathrm{C}$ & 3.57134 & 2.81459 & 0.24189 \\
\hline $\mathrm{H}$ & 5.44408 & 2.06237 & 1.07536 \\
\hline $\mathrm{C}$ & 3.84429 & 4.16336 & 0.45757 \\
\hline $\mathrm{H}$ & 4.775 & 4.45833 & 0.93826 \\
\hline $\mathrm{C}$ & 2.92591 & 5.14424 & 0.05582 \\
\hline $\mathrm{C}$ & 1.42291 & 3.47385 & -0.80659 \\
\hline $\mathrm{C}$ & 1.70993 & 4.79792 & -0.58235 \\
\hline $\mathrm{H}$ & 1.0308 & 5.59133 & -0.87411 \\
\hline $\mathrm{H}$ & 0.49191 & 3.17629 & -1.28133 \\
\hline $\mathrm{O}$ & 3.14634 & 6.45714 & 0.24747 \\
\hline $\mathrm{H}$ & 3.99654 & 6.59546 & 0.69776 \\
\hline $\mathrm{N}$ & -2.16104 & 0.05774 & -0.22385 \\
\hline $\mathrm{S}$ & -2.18692 & 0.65972 & -1.75182 \\
\hline $\mathrm{S}$ & -3.50632 & -0.48208 & 0.58756 \\
\hline $\mathrm{O}$ & -3.45674 & 1.23594 & -2.17429 \\
\hline $\mathrm{O}$ & -0.93521 & 1.41959 & -1.92582 \\
\hline $\mathrm{O}$ & -4.61475 & -0.87635 & -0.27401 \\
\hline $\mathrm{O}$ & -2.99659 & -1.36848 & 1.63593 \\
\hline $\mathrm{C}$ & -4.04649 & 1.0688 & 1.48351 \\
\hline $\mathrm{C}$ & -1.90944 & -0.86677 & -2.80039 \\
\hline $\mathrm{F}$ & -2.84826 & -1.77824 & -2.57122 \\
\hline $\mathrm{F}$ & -1.92093 & -0.52221 & -4.08914 \\
\hline $\mathrm{F}$ & -0.70496 & -1.39787 & -2.50803 \\
\hline $\mathrm{F}$ & -3.07155 & 1.47182 & 2.30837 \\
\hline $\mathrm{F}$ & -4.31285 & 2.04383 & 0.61493 \\
\hline $\mathrm{F}$ & -5.1396 & 0.79728 & 2.19822 \\
\hline $\mathrm{C}$ & 3.9093 & -3.83837 & -1.81147 \\
\hline $\mathrm{H}$ & 4.89863 & -3.37425 & -1.72449 \\
\hline $\mathrm{H}$ & 4.05879 & -4.88847 & -2.08164 \\
\hline $\mathrm{H}$ & 3.36847 & -3.36245 & -2.6369 \\
\hline $\mathrm{C}$ & 1.73746 & -4.40208 & -0.66627 \\
\hline
\end{tabular}

S64 


$\begin{array}{lllc}\mathrm{H} & 1.1815 & -4.38337 & 0.2747 \\ \mathrm{H} & 1.15898 & -3.8612 & -1.42525 \\ \mathrm{H} & 1.82814 & -5.44442 & -0.99222 \\ \mathrm{C} & 3.93814 & -4.53347 & 0.58869 \\ \mathrm{H} & 4.06374 & -5.57852 & 0.28311 \\ \mathrm{H} & 4.93341 & -4.08905 & 0.70598 \\ \mathrm{H} & 3.42759 & -4.50413 & 1.55162\end{array}$

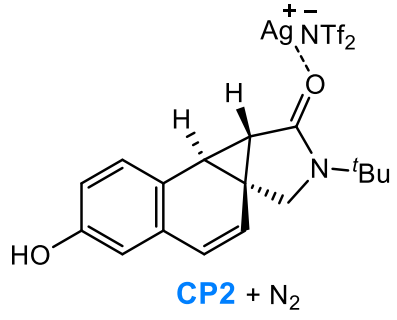

(Figure 2)

Zero-point correction $=$

Thermal correction to Energy=

Thermal correction to Enthalpy=

Thermal correction to Gibbs Free Energy=

Sum of electronic and zero-point Energies=

Sum of electronic and thermal Energies=

Sum of electronic and thermal Enthalpies=

Sum of electronic and thermal Free Energies=
0.389350 (Hartree/Particle)

0.429881

0.430825

0.304287

$-2946.484797$

$-2946.444267$

$-2946.443323$

$-2946.569861$

\section{Cartesian Coordinates}

Atom

$\mathrm{X}$

$\mathrm{Y}$

$\mathrm{Z}$

Ag

C

$\mathrm{H}$

C

$\mathrm{O}$

$\mathrm{N}$

C

$$
\begin{array}{ccc}
-0.44564 & -1.24761 & 0.62415 \\
2.66284 & 0.13886 & 0.69992 \\
2.1638 & 0.59769 & 1.54676 \\
2.56962 & -1.3293 & 0.52109 \\
1.58968 & -2.03996 & 0.85927 \\
3.69578 & -1.80488 & -0.05978 \\
3.91375 & -3.24889 & -0.41276
\end{array}
$$




\begin{tabular}{|c|c|c|c|}
\hline $\mathrm{C}$ & 4.74559 & -0.77336 & -0.16033 \\
\hline $\mathrm{H}$ & 5.5674 & -0.99514 & 0.53463 \\
\hline $\mathrm{H}$ & 5.15976 & -0.73675 & -1.17183 \\
\hline $\mathrm{N}$ & -1.04643 & -1.73659 & 4.62437 \\
\hline $\mathrm{N}$ & -0.63866 & -1.29289 & 3.69863 \\
\hline $\mathrm{C}$ & 4.05896 & 0.53409 & 0.22208 \\
\hline $\mathrm{C}$ & 2.84534 & 0.92966 & -0.59889 \\
\hline $\mathrm{C}$ & 4.83764 & 1.61438 & 0.85837 \\
\hline $\mathrm{C}$ & 2.47337 & 2.36841 & -0.6308 \\
\hline $\mathrm{H}$ & 2.63514 & 0.3702 & -1.50957 \\
\hline $\mathrm{C}$ & 4.42718 & 2.89569 & 0.81955 \\
\hline $\mathrm{H}$ & 5.7594 & 1.34114 & 1.36717 \\
\hline $\mathrm{C}$ & 3.22962 & 3.31893 & 0.09763 \\
\hline $\mathrm{H}$ & 5.01052 & 3.66643 & 1.31742 \\
\hline $\mathrm{C}$ & 2.85153 & 4.66783 & 0.07705 \\
\hline $\mathrm{H}$ & 3.41866 & 5.40973 & 0.63159 \\
\hline $\mathrm{C}$ & 1.74156 & 5.08118 & -0.66112 \\
\hline $\mathrm{C}$ & 1.37545 & 2.8007 & -1.38022 \\
\hline $\mathrm{C}$ & 1.00449 & 4.14381 & -1.3963 \\
\hline $\mathrm{H}$ & 0.13575 & 4.45864 & -1.97094 \\
\hline $\mathrm{H}$ & 0.78191 & 2.07689 & -1.93199 \\
\hline $\mathrm{O}$ & 1.42648 & 6.40915 & -0.63225 \\
\hline $\mathrm{H}$ & 0.63182 & 6.55808 & -1.16848 \\
\hline $\mathrm{N}$ & -2.29338 & -0.40421 & -0.13247 \\
\hline $\mathrm{S}$ & -3.59178 & -0.27187 & 0.89108 \\
\hline $\mathrm{S}$ & -2.26209 & 0.14128 & -1.69324 \\
\hline $\mathrm{O}$ & -4.87628 & -0.15341 & 0.21262 \\
\hline $\mathrm{O}$ & -3.36524 & -1.26227 & 1.94633 \\
\hline $\mathrm{O}$ & -3.16091 & 1.25642 & -1.96767 \\
\hline $\mathrm{O}$ & -0.83949 & 0.21084 & -2.0602 \\
\hline $\mathrm{C}$ & -2.91159 & -1.33042 & -2.64964 \\
\hline $\mathrm{C}$ & -3.26395 & 1.37512 & 1.71659 \\
\hline $\mathrm{F}$ & -3.23974 & 2.3595 & 0.82113 \\
\hline
\end{tabular}




$\begin{array}{lccc}\text { F } & -4.22203 & 1.60998 & 2.61418 \\ \text { F } & -2.07842 & 1.32825 & 2.34641 \\ \text { F } & -2.10728 & -2.3825 & -2.44331 \\ \text { F } & -4.14473 & -1.63907 & -2.26082 \\ \text { F } & -2.91249 & -1.03118 & -3.95035 \\ \text { C } & 5.2506 & -3.39919 & -1.15917 \\ \mathrm{H} & 6.10524 & -3.0733 & -0.55748 \\ \mathrm{H} & 5.39863 & -4.45821 & -1.39107 \\ \mathrm{H} & 5.25605 & -2.85054 & -2.10687 \\ \mathrm{C} & 2.77887 & -3.72988 & -1.33728 \\ \mathrm{H} & 1.80987 & -3.68751 & -0.83817 \\ \mathrm{H} & 2.73364 & -3.11807 & -2.24516 \\ \mathrm{H} & 2.96838 & -4.76664 & -1.63553 \\ \mathrm{C} & 3.95653 & -4.07535 & 0.88755 \\ \mathrm{H} & 4.12195 & -5.13234 & 0.65192 \\ \mathrm{H} & 4.77528 & -3.74081 & 1.53514 \\ \mathrm{H} & 3.01653 & -3.98679 & 1.43684\end{array}$

\section{Calculation of Axial Rotation of 9a and 11a}

The molecular structure optimizations were carried out using the hybrid density functional method based on $\omega$ B97XD functional and the 6-311+G* basis set for $\mathrm{H}, \mathrm{C}, \mathrm{N}, \mathrm{O}$, and $\mathrm{F}$. The vibrational frequencies were computed at the same level to check whether each optimized structure is at an energy minimum on the potential energy surfaces (no imaginary frequency) or a transition state (one imaginary frequency) and to evaluate its zero-point vibrational energy (ZPVE) and thermal corrections at $298.15 \mathrm{~K}$. The intrinsic reaction coordinate (IRC) method was used to track minimum energy paths from transition structures to the corresponding local minima. ${ }^{[5]}$ 


\section{Figure S3}
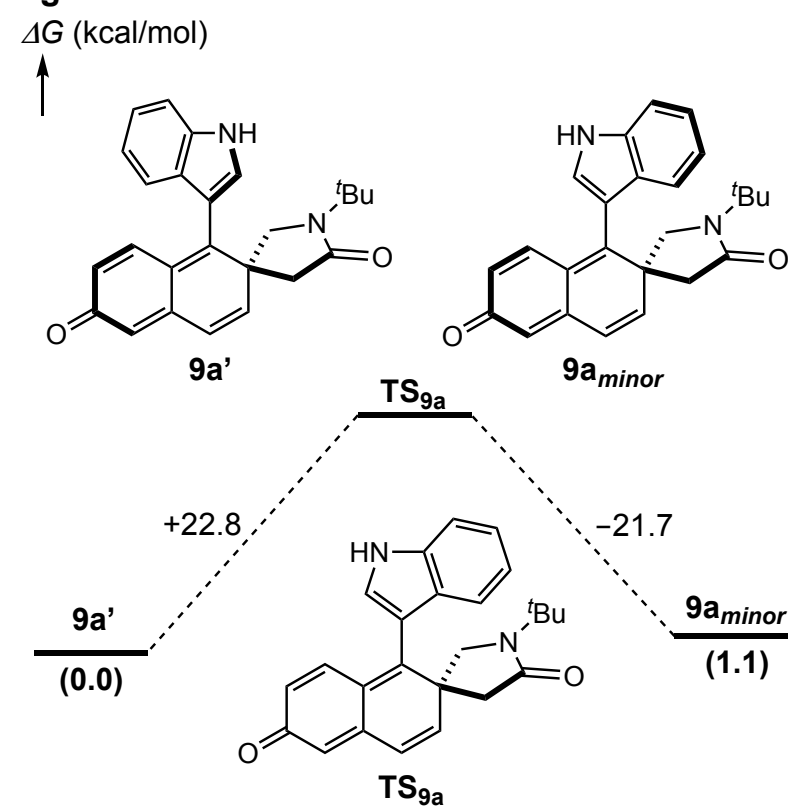

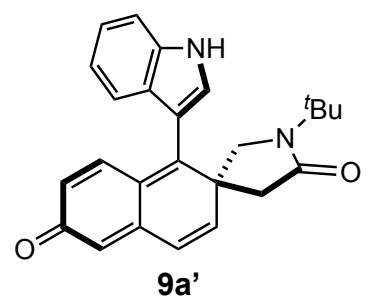

(Figure S4)

Zero-point correction=

Thermal correction to Energy=

Thermal correction to Enthalpy=

Thermal correction to Gibbs Free Energy=

Sum of electronic and zero-point Energies=

Sum of electronic and thermal Energies=

Sum of electronic and thermal Enthalpies=

Sum of electronic and thermal Free Energies=
0.440038 (Hartree/Particle)

0.464539

0.465484

0.385051

$-1226.506488$

$-1226.481987$

$-1226.481043$

$-1226.561476$

\section{Cartesian Coordinates}

Atom

$\mathrm{X}$

$\mathrm{Y}$

Z

$\mathrm{O}$

$\begin{array}{lll}-3.59463 & -1.59654 & 1.96351\end{array}$

$\mathrm{O}$

$\begin{array}{lll}5.84025 & -2.33083 & -0.69463\end{array}$ 
Supporting Information

\begin{tabular}{|c|c|c|c|}
\hline $\mathrm{N}$ & -2.69622 & -1.00913 & -0.09089 \\
\hline $\mathrm{C}$ & 3.31718 & -0.00991 & 0.31161 \\
\hline $\mathrm{H}$ & 3.1976 & 1.01846 & 0.63646 \\
\hline $\mathrm{C}$ & 0.68505 & 2.19813 & -0.02922 \\
\hline $\mathrm{C}$ & 0.51451 & 3.37165 & 0.73533 \\
\hline $\mathrm{C}$ & 3.51483 & -2.70467 & -0.5257 \\
\hline $\mathrm{H}$ & 3.65165 & -3.73134 & -0.85403 \\
\hline $\mathrm{C}$ & -1.41023 & -0.52138 & -0.57153 \\
\hline $\mathrm{H}$ & -1.36018 & 0.57408 & -0.53062 \\
\hline $\mathrm{H}$ & -1.21538 & -0.83706 & -1.5986 \\
\hline $\mathrm{C}$ & 2.11184 & -0.83088 & 0.20951 \\
\hline $\mathrm{C}$ & 0.69852 & 3.54058 & -2.01377 \\
\hline $\mathrm{H}$ & 0.77464 & 3.63363 & -3.0926 \\
\hline $\mathrm{C}$ & 0.78236 & 2.29228 & -1.42523 \\
\hline $\mathrm{H}$ & 0.93073 & 1.39793 & -2.02426 \\
\hline $\mathrm{C}$ & -1.20385 & -1.22526 & 1.70439 \\
\hline $\mathrm{H}$ & -0.93817 & -2.05913 & 2.35691 \\
\hline $\mathrm{H}$ & -1.11463 & -0.29623 & 2.27628 \\
\hline $\mathrm{C}$ & 0.52138 & 4.70044 & -1.23395 \\
\hline $\mathrm{H}$ & 0.46021 & 5.66646 & -1.72558 \\
\hline $\mathrm{C}$ & 1.09271 & -3.04962 & -0.34748 \\
\hline $\mathrm{H}$ & 1.22228 & -4.07641 & -0.6789 \\
\hline $\mathrm{C}$ & -4.86923 & -1.97583 & -0.72302 \\
\hline $\mathrm{H}$ & -5.79758 & -1.78996 & -1.27379 \\
\hline $\mathrm{H}$ & -5.11262 & -2.21449 & 0.31256 \\
\hline $\mathrm{H}$ & -4.36763 & -2.83837 & -1.17499 \\
\hline $\mathrm{C}$ & -0.37778 & -1.16785 & 0.39948 \\
\hline $\mathrm{C}$ & 0.42862 & 4.6359 & 0.14521 \\
\hline $\mathrm{H}$ & 0.2962 & 5.53231 & 0.74361 \\
\hline $\mathrm{C}$ & 0.72479 & 1.08868 & 0.89242 \\
\hline $\mathrm{C}$ & -2.65147 & -1.32199 & 1.24242 \\
\hline $\mathrm{C}$ & -4.65236 & 0.48025 & -0.16341 \\
\hline $\mathrm{H}$ & -4.00313 & 1.36163 & -0.22131 \\
\hline
\end{tabular}




$\begin{array}{lccc}\mathrm{H} & -4.88304 & 0.27917 & 0.88543 \\ \mathrm{H} & -5.58585 & 0.71012 & -0.68809 \\ \mathrm{C} & 4.53441 & -0.49171 & 0.01688 \\ \mathrm{H} & 5.42679 & 0.1215 & 0.09304 \\ \mathrm{C} & -3.96761 & -0.73638 & -0.80389 \\ \mathrm{C} & -3.67444 & -0.44159 & -2.27936 \\ \mathrm{H} & -3.0613 & 0.45617 & -2.41015 \\ \mathrm{H} & -4.62299 & -0.27182 & -2.79745 \\ \mathrm{H} & -3.17424 & -1.28561 & -2.76629 \\ \mathrm{C} & 4.73131 & -1.88656 & -0.42653 \\ \mathrm{C} & 2.28683 & -2.22317 & -0.23197 \\ \mathrm{C} & 0.59254 & 1.62671 & 2.14423 \\ \mathrm{H} & 0.60304 & 1.13681 & 3.10784 \\ \mathrm{C} & -0.11986 & -2.57415 & -0.06422 \\ \mathrm{H} & -0.99819 & -3.20783 & -0.16566 \\ \mathrm{C} & 0.88453 & -0.33414 & 0.51033 \\ \mathrm{~N} & 0.46488 & 2.99297 & 2.05705 \\ \mathrm{H} & 0.35883 & 3.61492 & 2.84081\end{array}$

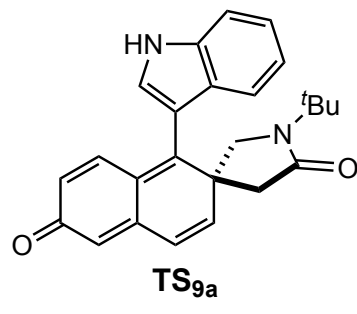

(Figure S4)

Zero-point correction= 0.441555 (Hartree/Particle)

Thermal correction to Energy= 0.464633

Thermal correction to Enthalpy= 0.465578

Thermal correction to Gibbs Free Energy= 0.390535

Sum of electronic and zero-point Energies= $-1226.474182$

Sum of electronic and thermal Energies= $-1226.451104$

Sum of electronic and thermal Enthalpies= $-1226.450160$

Sum of electronic and thermal Free Energies= $-1226.525202$ 
Cartesian Coordinates

\begin{tabular}{|c|c|c|c|}
\hline Atom & $\mathrm{X}$ & $\mathrm{Y}$ & $\mathrm{Z}$ \\
\hline $\mathrm{O}$ & -3.00083 & -1.5689 & 2.02116 \\
\hline $\mathrm{O}$ & 6.36581 & -1.05285 & -1.02103 \\
\hline $\mathrm{N}$ & -1.90419 & -1.65804 & -0.01521 \\
\hline $\mathrm{C}$ & 3.65185 & 0.98479 & 0.11 \\
\hline $\mathrm{H}$ & 3.52199 & 2.0094 & 0.40055 \\
\hline $\mathrm{C}$ & -0.79755 & 2.17487 & 0.01831 \\
\hline $\mathrm{C}$ & -0.76382 & 3.58474 & -0.10382 \\
\hline $\mathrm{C}$ & 4.26112 & -1.71817 & -0.16399 \\
\hline $\mathrm{H}$ & 4.55817 & -2.763 & -0.18599 \\
\hline $\mathrm{C}$ & -0.69206 & -1.12085 & -0.61832 \\
\hline $\mathrm{H}$ & -0.88984 & -0.23438 & -1.23183 \\
\hline $\mathrm{H}$ & -0.20004 & -1.85826 & -1.2551 \\
\hline $\mathrm{C}$ & 2.54671 & 0.03449 & 0.25925 \\
\hline $\mathrm{C}$ & -3.20762 & 2.40791 & -0.24899 \\
\hline $\mathrm{H}$ & -4.17981 & 1.92703 & -0.29825 \\
\hline $\mathrm{C}$ & -2.08989 & 1.61385 & -0.06563 \\
\hline $\mathrm{H}$ & -2.26798 & 0.55744 & 0.0133 \\
\hline $\mathrm{C}$ & -0.79368 & -0.57249 & 1.75988 \\
\hline $\mathrm{H}$ & -0.43668 & -0.97159 & 2.71193 \\
\hline $\mathrm{H}$ & -1.05092 & 0.47512 & 1.92474 \\
\hline $\mathrm{C}$ & -3.11457 & 3.80192 & -0.36249 \\
\hline $\mathrm{H}$ & -4.00833 & 4.40076 & -0.50426 \\
\hline $\mathrm{C}$ & 2.12092 & -2.41418 & 0.70411 \\
\hline $\mathrm{H}$ & 2.52827 & -3.40792 & 0.86519 \\
\hline $\mathrm{C}$ & -3.40901 & -3.60415 & -0.13754 \\
\hline $\mathrm{H}$ & -4.18277 & -4.09245 & -0.73963 \\
\hline $\mathrm{H}$ & -3.79681 & -3.43364 & 0.86711 \\
\hline $\mathrm{H}$ & -2.54755 & -4.27796 & -0.07397 \\
\hline $\mathrm{C}$ & 0.2161 & -0.80847 & 0.6143 \\
\hline
\end{tabular}




\begin{tabular}{|c|c|c|c|}
\hline $\mathrm{C}$ & -1.877 & 4.40553 & -0.29018 \\
\hline $\mathrm{H}$ & -1.76008 & 5.48203 & -0.37453 \\
\hline $\mathrm{C}$ & 0.60715 & 1.71196 & 0.19259 \\
\hline $\mathrm{C}$ & -2.03953 & -1.32829 & 1.31233 \\
\hline $\mathrm{C}$ & -4.1886 & -1.31292 & -0.86911 \\
\hline $\mathrm{H}$ & -3.9093 & -0.39707 & -1.40278 \\
\hline $\mathrm{H}$ & -4.52721 & -1.05289 & 0.13688 \\
\hline $\mathrm{H}$ & -5.0217 & -1.77714 & -1.40745 \\
\hline $\mathrm{C}$ & 4.89169 & 0.64814 & -0.28428 \\
\hline $\mathrm{H}$ & 5.67513 & 1.39542 & -0.36635 \\
\hline $\mathrm{C}$ & -2.99807 & -2.28199 & -0.80095 \\
\hline $\mathrm{C}$ & -2.50771 & -2.58 & -2.22223 \\
\hline $\mathrm{H}$ & -2.18914 & -1.67485 & -2.74938 \\
\hline $\mathrm{H}$ & -3.33313 & -3.01957 & -2.79006 \\
\hline $\mathrm{H}$ & -1.68257 & -3.29967 & -2.22483 \\
\hline $\mathrm{C}$ & 5.27138 & -0.74456 & -0.56435 \\
\hline $\mathrm{C}$ & 3.01785 & -1.3708 & 0.24803 \\
\hline $\mathrm{C}$ & 1.33388 & 2.89353 & 0.14895 \\
\hline $\mathrm{H}$ & 2.38416 & 3.08398 & 0.21626 \\
\hline $\mathrm{C}$ & 0.84316 & -2.14209 & 0.9203 \\
\hline $\mathrm{H}$ & 0.1566 & -2.91503 & 1.25676 \\
\hline $\mathrm{C}$ & 1.19859 & 0.34439 & 0.35739 \\
\hline $\mathrm{N}$ & 0.54362 & 3.97915 & -0.01285 \\
\hline $\mathrm{H}$ & 0.87603 & 4.92738 & -0.07429 \\
\hline
\end{tabular}

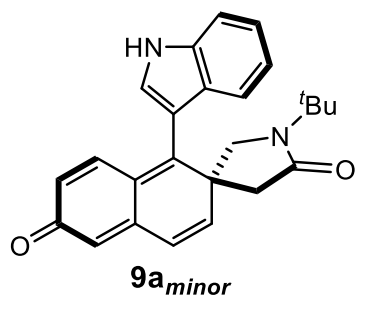

(Figure S4)

Zero-point correction $=$ 0.440237 (Hartree/Particle) Thermal correction to Energy= 0.464653 
Thermal correction to Enthalpy= 0.465597

Thermal correction to Gibbs Free Energy= 0.385441

Sum of electronic and zero-point Energies= $-1226.504993$

Sum of electronic and thermal Energies= $-1226.480577$

Sum of electronic and thermal Enthalpies= $-1226.479632$

Sum of electronic and thermal Free Energies=

\section{Cartesian Coordinates}

$\begin{array}{lccc}\text { Atom } & \mathrm{X} & \mathrm{Y} & \mathrm{Z} \\ \mathrm{O} & 3.48166 & 0.41467 & -2.10443 \\ \mathrm{O} & -5.18087 & -3.56154 & 0.20367 \\ \mathrm{~N} & 2.96022 & -0.59571 & -0.08542 \\ \mathrm{C} & -3.11062 & -0.69634 & 0.72386 \\ \mathrm{H} & -3.15345 & 0.31587 & 1.11215 \\ \mathrm{C} & -1.30509 & 2.13002 & -0.06628 \\ \mathrm{C} & -1.22745 & 3.27504 & 0.75396 \\ \mathrm{C} & -2.89135 & -3.31627 & -0.31975 \\ \mathrm{H} & -2.86811 & -4.32909 & -0.71249 \\ \mathrm{C} & 1.75951 & -0.76766 & 0.72097 \\ \mathrm{H} & 1.61484 & 0.08498 & 1.39657 \\ \mathrm{H} & 1.79783 & -1.68191 & 1.31655 \\ \mathrm{C} & -1.82617 & -1.17033 & 0.21091 \\ \mathrm{C} & -2.22113 & 3.49128 & -1.81156 \\ \mathrm{H} & -2.61923 & 3.6013 & -2.81531 \\ \mathrm{C} & -1.81509 & 2.2467 & -1.36765 \\ \mathrm{H} & -1.89219 & 1.37046 & -2.00521 \\ \mathrm{C} & 1.16556 & 0.08095 & -1.43511 \\ \mathrm{H} & 0.84799 & -0.20057 & -2.44105 \\ \mathrm{H} & 0.87602 & 1.12388 & -1.26697 \\ \mathrm{C} & -2.12972 & 4.62471 & -0.9797 \\ \mathrm{H} & -2.45674 & 5.58833 & -1.35792 \\ \mathrm{C} & -0.51446 & -3.02225 & -0.84828\end{array}$


Supporting Information

\begin{tabular}{|c|c|c|c|}
\hline $\mathrm{H}$ & -0.48441 & -4.035 & -1.24116 \\
\hline $\mathrm{C}$ & 5.28085 & -1.32233 & -0.46427 \\
\hline $\mathrm{H}$ & 6.28295 & -1.35931 & -0.02358 \\
\hline $\mathrm{H}$ & 5.3329 & -0.80395 & -1.42181 \\
\hline $\mathrm{H}$ & 4.94278 & -2.34971 & -0.63765 \\
\hline $\mathrm{C}$ & 0.61364 & -0.84978 & -0.32822 \\
\hline $\mathrm{C}$ & -1.63549 & 4.536 & 0.30954 \\
\hline $\mathrm{H}$ & -1.56959 & 5.41078 & 0.94954 \\
\hline $\mathrm{C}$ & -0.80496 & 1.01681 & 0.70381 \\
\hline $\mathrm{C}$ & 2.67754 & -0.00465 & -1.29129 \\
\hline $\mathrm{C}$ & 4.77799 & 0.84191 & 0.7407 \\
\hline $\mathrm{H}$ & 4.08982 & 1.35179 & 1.4252 \\
\hline $\mathrm{H}$ & 4.81122 & 1.39447 & -0.2011 \\
\hline $\mathrm{H}$ & 5.77705 & 0.856 & 1.18935 \\
\hline $\mathrm{C}$ & -4.20756 & -1.46923 & 0.72242 \\
\hline $\mathrm{H}$ & -5.15903 & -1.11332 & 1.10475 \\
\hline $\mathrm{C}$ & 4.32322 & -0.6049 & 0.49732 \\
\hline $\mathrm{C}$ & 4.3082 & -1.36865 & 1.82632 \\
\hline $\mathrm{H}$ & 3.67151 & -0.88703 & 2.57601 \\
\hline $\mathrm{H}$ & 5.32599 & -1.3954 & 2.22672 \\
\hline $\mathrm{H}$ & 3.97445 & -2.40312 & 1.69197 \\
\hline $\mathrm{C}$ & -4.18389 & -2.85084 & 0.20145 \\
\hline $\mathrm{C}$ & -1.78447 & -2.54109 & -0.32155 \\
\hline $\mathrm{C}$ & -0.46556 & 1.52211 & 1.92994 \\
\hline $\mathrm{H}$ & -0.08381 & 1.01131 & 2.80291 \\
\hline $\mathrm{C}$ & 0.57712 & -2.25752 & -0.85425 \\
\hline $\mathrm{H}$ & 1.51625 & -2.63943 & -1.24859 \\
\hline $\mathrm{C}$ & -0.71967 & -0.38312 & 0.22403 \\
\hline $\mathrm{N}$ & -0.7136 & 2.87412 & 1.96592 \\
\hline $\mathrm{H}$ & -0.55328 & 3.47119 & 2.75965 \\
\hline
\end{tabular}




\section{Figure S4}
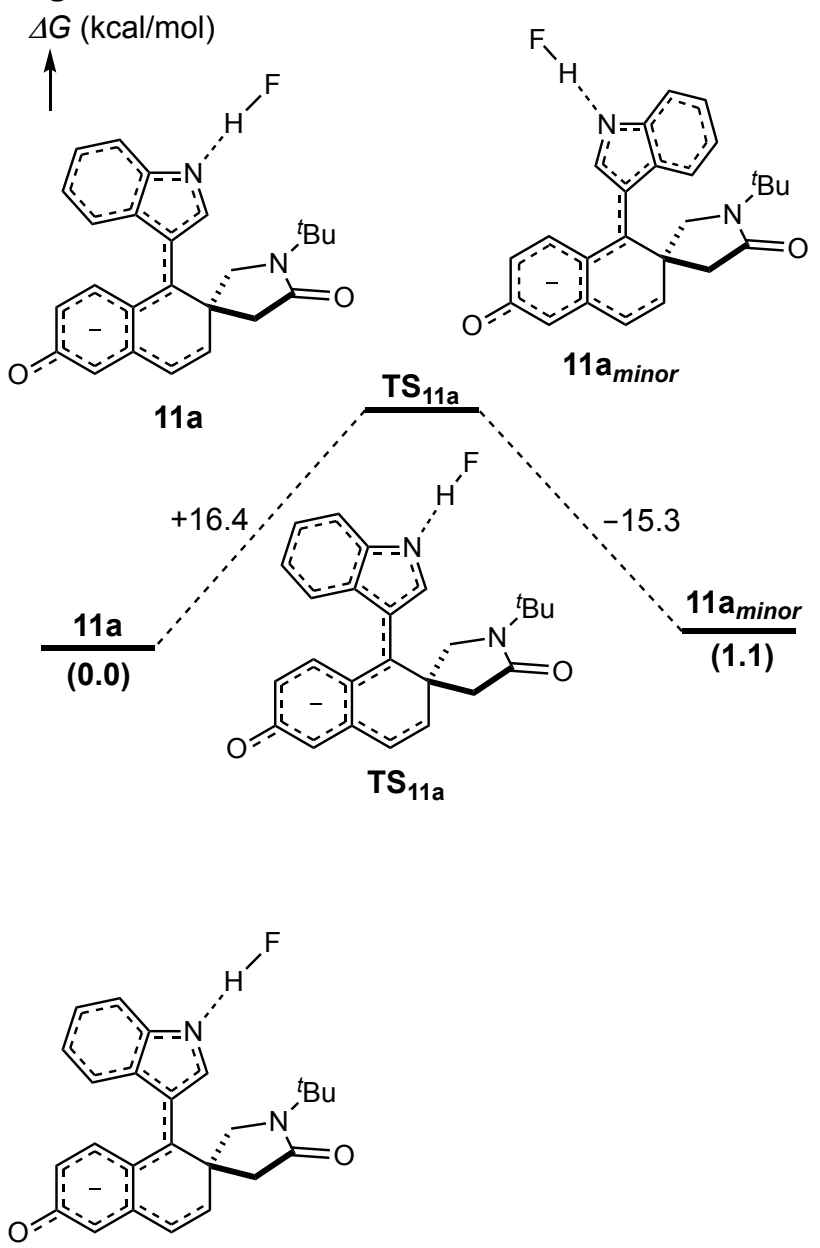

11a (Figure S4)

Zero-point correction=

Thermal correction to Energy=

Thermal correction to Enthalpy=

Thermal correction to Gibbs Free Energy=

Sum of electronic and zero-point Energies=

Sum of electronic and thermal Energies=

Sum of electronic and thermal Enthalpies=

0.438313 (Hartree/Particle)

Sum of electronic and thermal Free Energies=

0.464260

0.465204

0.381072

$-1326.457501$

$-1326.431554$

$-1326.430609$

$-1326.514741$

\section{Cartesian Coordinates}

Atom

$\mathrm{X}$

$\mathrm{Y}$

Z

$\mathrm{O}$

$\begin{array}{lll}3.13664 & -2.53287 & -1.70496\end{array}$ 


\begin{tabular}{|c|c|c|c|}
\hline $\mathrm{O}$ & -6.24052 & -1.86716 & -0.13849 \\
\hline $\mathrm{N}$ & 2.57182 & -1.22476 & 0.12246 \\
\hline $\mathrm{C}$ & -3.23497 & -0.01827 & -0.81756 \\
\hline $\mathrm{H}$ & -2.90314 & 0.87037 & -1.34509 \\
\hline $\mathrm{C}$ & -1.04406 & 2.14813 & -0.00512 \\
\hline $\mathrm{C}$ & -0.0668 & 3.1395 & -0.29036 \\
\hline $\mathrm{C}$ & -4.00699 & -2.39863 & 0.43451 \\
\hline $\mathrm{H}$ & -4.34037 & -3.32426 & 0.89841 \\
\hline $\mathrm{C}$ & 1.39593 & -0.99548 & 0.95066 \\
\hline $\mathrm{H}$ & 1.28053 & 0.06139 & 1.18901 \\
\hline $\mathrm{H}$ & 1.48711 & -1.54488 & 1.89802 \\
\hline $\mathrm{C}$ & -2.22797 & -0.82523 & -0.1742 \\
\hline $\mathrm{C}$ & -2.42122 & 3.86931 & 0.96331 \\
\hline $\mathrm{H}$ & -3.33846 & 4.16717 & 1.4653 \\
\hline $\mathrm{C}$ & -2.22262 & 2.53229 & 0.65287 \\
\hline $\mathrm{H}$ & -2.97758 & 1.79847 & 0.91708 \\
\hline $\mathrm{C}$ & 0.81577 & -2.02425 & -1.19615 \\
\hline $\mathrm{H}$ & 0.48844 & -3.03005 & -1.46757 \\
\hline $\mathrm{H}$ & 0.57686 & -1.36533 & -2.03672 \\
\hline $\mathrm{C}$ & -1.46146 & 4.84454 & 0.64147 \\
\hline $\mathrm{H}$ & -1.64805 & 5.88574 & 0.89288 \\
\hline $\mathrm{C}$ & -1.688 & -2.96916 & 0.9839 \\
\hline $\mathrm{H}$ & -2.03971 & -3.86885 & 1.4834 \\
\hline $\mathrm{C}$ & 4.59236 & -2.10507 & 1.18402 \\
\hline $\mathrm{H}$ & 5.59949 & -1.86872 & 1.54603 \\
\hline $\mathrm{H}$ & 4.66592 & -2.89408 & 0.43012 \\
\hline $\mathrm{H}$ & 4.00452 & -2.47961 & 2.03114 \\
\hline $\mathrm{C}$ & 0.17399 & -1.52432 & 0.1344 \\
\hline $\mathrm{C}$ & -0.27618 & 4.48585 & 0.02025 \\
\hline $\mathrm{H}$ & 0.49012 & 5.22014 & -0.21297 \\
\hline $\mathrm{C}$ & -0.44022 & 0.88487 & -0.4005 \\
\hline $\mathrm{C}$ & 2.31803 & -1.98939 & -0.97744 \\
\hline $\mathrm{C}$ & 4.75516 & -0.31698 & -0.60494 \\
\hline
\end{tabular}




$\begin{array}{lccl}\mathrm{H} & 4.31092 & 0.61469 & -0.96932 \\ \mathrm{H} & 4.82306 & -1.0463 & -1.412 \\ \mathrm{H} & 5.76632 & -0.08524 & -0.25012 \\ \mathrm{C} & -4.54697 & -0.35274 & -0.82511 \\ \mathrm{H} & -5.28018 & 0.25691 & -1.34535 \\ \mathrm{C} & 3.93401 & -0.85881 & 0.57556 \\ \mathrm{C} & 3.84111 & 0.27071 & 1.61032 \\ \mathrm{H} & 3.42036 & 1.17339 & 1.15523 \\ \mathrm{H} & 4.85551 & 0.5141 & 1.94332 \\ \mathrm{H} & 3.26104 & -0.01057 & 2.4956 \\ \mathrm{C} & -5.04312 & -1.5614 & -0.16525 \\ \mathrm{C} & -2.68593 & -2.06931 & 0.41504 \\ \mathrm{C} & 0.838 & 1.26792 & -0.87873 \\ \mathrm{H} & 1.61588 & 0.61468 & -1.25618 \\ \mathrm{C} & -0.38589 & -2.72855 & 0.84699 \\ \mathrm{H} & 0.3526 & -3.44202 & 1.21063 \\ \mathrm{C} & -0.88663 & -0.45126 & -0.15437 \\ \mathrm{~N} & 1.06726 & 2.57406 & -0.84137 \\ \mathrm{H} & 2.58759 & 2.85762 & -0.80407 \\ \mathrm{~F} & 3.57186 & 2.79499 & -0.69091\end{array}$

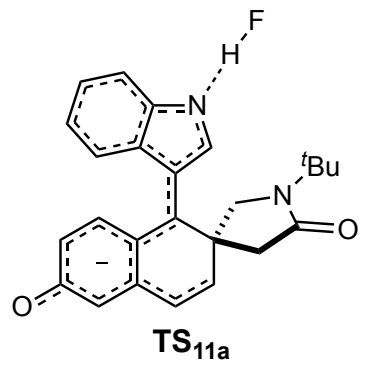

(Figure S4)

Zero-point correction=

Thermal correction to Energy=

Thermal correction to Enthalpy=

Thermal correction to Gibbs Free Energy=

Sum of electronic and zero-point Energies=

Sum of electronic and thermal Energies=
0.439236 (Hartree/Particle)

$$
0.464418
$$

0.465363

0.383143

$-1326.432471$

$-1326.407289$ 
Sum of electronic and thermal Enthalpies=

$-1326.406344$

Sum of electronic and thermal Free Energies=

$-1326.488564$

\section{Cartesian Coordinates}

\begin{tabular}{lccl} 
Atom & $\mathrm{X}$ & $\mathrm{Y}$ & $\mathrm{Z}$ \\
\hline $\mathrm{O}$ & -3.37508 & -1.69624 & 2.12647 \\
$\mathrm{O}$ & 6.0245 & -2.72506 & -0.505 \\
$\mathrm{~N}$ & -2.65725 & -1.11467 & -0.00956 \\
$\mathrm{C}$ & 3.45104 & -0.2402 & -0.01812 \\
$\mathrm{H}$ & 3.36755 & 0.81744 & 0.09422 \\
$\mathrm{C}$ & 1.32258 & 2.36101 & 0.15493 \\
$\mathrm{C}$ & 0.26054 & 3.33201 & 0.11907 \\
$\mathrm{C}$ & 3.67275 & -2.98368 & -0.282 \\
$\mathrm{H}$ & 3.77448 & -4.06265 & -0.38233 \\
$\mathrm{C}$ & -1.40215 & -1.10994 & -0.76589 \\
$\mathrm{H}$ & -1.2569 & -0.17505 & -1.30749 \\
$\mathrm{H}$ & -1.39897 & -1.92071 & -1.50632 \\
$\mathrm{C}$ & 2.2308 & -0.99191 & 0.03329 \\
$\mathrm{C}$ & 2.82482 & 4.29632 & 0.00117 \\
$\mathrm{H}$ & 3.84764 & 4.66582 & -0.0243 \\
$\mathrm{C}$ & 2.62118 & 2.92058 & 0.11604 \\
$\mathrm{H}$ & 3.52579 & 2.33815 & 0.19853 \\
$\mathrm{C}$ & -1.01368 & -1.2746 & 1.66844 \\
$\mathrm{H}$ & -0.71447 & -2.0693 & 2.35653 \\
$\mathrm{H}$ & -0.84923 & -0.32154 & 2.17888 \\
$\mathrm{C}$ & 1.75704 & 5.19899 & -0.07147 \\
$\mathrm{H}$ & 1.94274 & 6.26658 & -0.16456 \\
$\mathrm{C}$ & 1.28105 & -3.31626 & -0.04707 \\
$\mathrm{H}$ & 1.45043 & -4.38579 & -0.15287 \\
$\mathrm{C}$ & -4.55749 & -2.42 & -0.88051 \\
$\mathrm{H}$ & -5.53603 & -2.37509 & -1.37403 \\
$\mathrm{H}$ & -4.6776 & -2.91833 & 0.08604
\end{tabular}


Supporting Information

\begin{tabular}{|c|c|c|c|}
\hline $\mathrm{H}$ & -3.89085 & -3.0261 & -1.50697 \\
\hline $\mathrm{C}$ & -0.26198 & -1.36164 & 0.30158 \\
\hline $\mathrm{C}$ & 0.46037 & 4.7091 & -0.00328 \\
\hline $\mathrm{H}$ & -0.40578 & 5.36528 & -0.03105 \\
\hline $\mathrm{C}$ & 0.6365 & 1.03383 & 0.26606 \\
\hline $\mathrm{C}$ & -2.49363 & -1.40646 & 1.32453 \\
\hline $\mathrm{C}$ & -4.94222 & -0.14168 & 0.17911 \\
\hline $\mathrm{H}$ & -4.53292 & 0.86793 & 0.28551 \\
\hline $\mathrm{H}$ & -5.10405 & -0.58208 & 1.1626 \\
\hline $\mathrm{H}$ & -5.90611 & -0.06464 & -0.33955 \\
\hline $\mathrm{C}$ & 4.69114 & -0.77507 & -0.18895 \\
\hline $\mathrm{H}$ & 5.5729 & -0.13865 & -0.21366 \\
\hline $\mathrm{C}$ & -3.98984 & -1.00223 & -0.67747 \\
\hline $\mathrm{C}$ & -3.81986 & -0.2763 & -2.02552 \\
\hline $\mathrm{H}$ & -3.45554 & 0.7436 & -1.86297 \\
\hline $\mathrm{H}$ & -4.80112 & -0.20787 & -2.50847 \\
\hline $\mathrm{H}$ & -3.15166 & -0.80573 & -2.71351 \\
\hline $\mathrm{C}$ & 4.90156 & -2.20926 & -0.34265 \\
\hline $\mathrm{C}$ & 2.42757 & -2.43289 & -0.10443 \\
\hline $\mathrm{C}$ & -0.74057 & 1.4405 & 0.34676 \\
\hline $\mathrm{H}$ & -1.60549 & 0.81366 & 0.46937 \\
\hline $\mathrm{C}$ & 0.0582 & -2.82675 & 0.1438 \\
\hline $\mathrm{H}$ & -0.80598 & -3.48767 & 0.20525 \\
\hline $\mathrm{C}$ & 0.9593 & -0.38432 & 0.194 \\
\hline $\mathrm{N}$ & -0.97703 & 2.73533 & 0.24209 \\
\hline $\mathrm{H}$ & -2.47176 & 2.93291 & -0.15071 \\
\hline $\mathrm{F}$ & -3.40918 & 2.76898 & -0.44958 \\
\hline
\end{tabular}




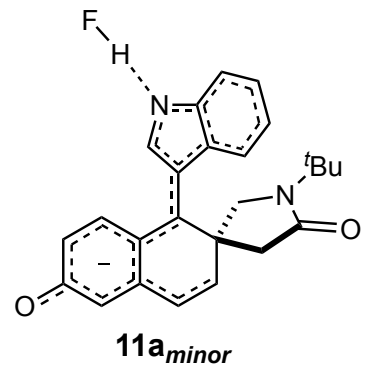

(Figure S4)
Zero-point correction $=$

Thermal correction to Energy=

Thermal correction to Enthalpy=

Thermal correction to Gibbs Free Energy=

Sum of electronic and zero-point Energies=

Sum of electronic and thermal Energies=

Sum of electronic and thermal Enthalpies=

Sum of electronic and thermal Free Energies=
0.438603 (Hartree/Particle)

0.464628

0.465573

0.381001

$-1326.455404$

$-1326.429378$

$-1326.428434$

$-1326.513006$

\section{Cartesian Coordinates}

\begin{tabular}{lccc} 
Atom & $\mathrm{X}$ & $\mathrm{Y}$ & $\mathrm{Z}$ \\
\hline $\mathrm{O}$ & -3.53885 & -0.65644 & 2.20213 \\
$\mathrm{O}$ & 5.7776 & -2.64158 & -0.89876 \\
$\mathrm{~N}$ & -2.69315 & -1.06244 & 0.08858 \\
$\mathrm{C}$ & 3.03259 & -0.3229 & -0.91247 \\
$\mathrm{H}$ & 2.76728 & 0.64958 & -1.31253 \\
$\mathrm{C}$ & 1.34491 & 2.09346 & 0.22187 \\
$\mathrm{C}$ & 0.51631 & 3.22096 & -0.032 \\
$\mathrm{C}$ & 3.60318 & -2.90554 & -0.00358 \\
$\mathrm{H}$ & 3.84705 & -3.9195 & 0.30567 \\
$\mathrm{C}$ & -1.44528 & -1.51491 & -0.50213 \\
$\mathrm{H}$ & -1.17594 & -0.92959 & -1.38384 \\
$\mathrm{H}$ & -1.51867 & -2.56435 & -0.817 \\
$\mathrm{C}$ & 2.02796 & -1.02967 & -0.15385 \\
$\mathrm{C}$ & 3.10289 & 3.60131 & 0.87915 \\
$\mathrm{H}$ & 4.11289 & 3.76459 & 1.24704
\end{tabular}


Supporting Information

\begin{tabular}{|c|c|c|c|}
\hline $\mathrm{C}$ & 2.64498 & 2.3041 & 0.70687 \\
\hline $\mathrm{H}$ & 3.29265 & 1.46412 & 0.94017 \\
\hline $\mathrm{C}$ & -1.14075 & -0.78015 & 1.81883 \\
\hline $\mathrm{H}$ & -0.97816 & -1.33161 & 2.74808 \\
\hline $\mathrm{H}$ & -0.8748 & 0.26507 & 1.99898 \\
\hline $\mathrm{C}$ & 2.2864 & 4.70796 & 0.58578 \\
\hline $\mathrm{H}$ & 2.67535 & 5.71377 & 0.72469 \\
\hline $\mathrm{C}$ & 1.33514 & -3.20464 & 0.86295 \\
\hline $\mathrm{H}$ & 1.60253 & -4.21356 & 1.16849 \\
\hline $\mathrm{C}$ & -4.81724 & -2.24129 & -0.24292 \\
\hline $\mathrm{H}$ & -5.7713 & -2.24564 & -0.78264 \\
\hline $\mathrm{H}$ & -5.01947 & -2.20316 & 0.83044 \\
\hline $\mathrm{H}$ & -4.28944 & -3.17541 & -0.47096 \\
\hline $\mathrm{C}$ & -0.3534 & -1.36991 & 0.62319 \\
\hline $\mathrm{C}$ & 0.98879 & 4.52594 & 0.13586 \\
\hline $\mathrm{H}$ & 0.33448 & 5.36837 & -0.07137 \\
\hline $\mathrm{C}$ & 0.50255 & 0.93189 & -0.00654 \\
\hline $\mathrm{C}$ & -2.60573 & -0.8287 & 1.43288 \\
\hline $\mathrm{C}$ & -4.71444 & 0.28561 & -0.36087 \\
\hline $\mathrm{H}$ & -4.11548 & 1.15844 & -0.64474 \\
\hline $\mathrm{H}$ & -4.96473 & 0.3595 & 0.69773 \\
\hline $\mathrm{H}$ & -5.64311 & 0.30665 & -0.94361 \\
\hline $\mathrm{C}$ & 4.25598 & -0.84029 & -1.17152 \\
\hline $\mathrm{H}$ & 4.98199 & -0.29472 & -1.76757 \\
\hline $\mathrm{C}$ & -3.97221 & -1.02786 & -0.65732 \\
\hline $\mathrm{C}$ & -3.68713 & -1.07783 & -2.16368 \\
\hline $\mathrm{H}$ & -3.10109 & -0.21058 & -2.48498 \\
\hline $\mathrm{H}$ & -4.64151 & -1.04914 & -2.69882 \\
\hline $\mathrm{H}$ & -3.16512 & -1.99304 & -2.46283 \\
\hline $\mathrm{C}$ & 4.65446 & -2.16661 & -0.69757 \\
\hline $\mathrm{C}$ & 2.36693 & -2.38733 & 0.23532 \\
\hline $\mathrm{C}$ & -0.75092 & 1.50198 & -0.34773 \\
\hline $\mathrm{H}$ & -1.66709 & 0.97951 & -0.58857 \\
\hline
\end{tabular}


Supporting Information

$\begin{array}{llll}\mathrm{C} & 0.09118 & -2.7546 & 1.00719 \\ \mathrm{H} & -0.68768 & -3.39299 & 1.42332 \\ \mathrm{C} & 0.78926 & -0.46477 & 0.13735 \\ \mathrm{~N} & -0.754 & 2.82678 & -0.40108 \\ \mathrm{H} & -2.18247 & 3.24683 & -0.91535 \\ \mathrm{~F} & -3.10405 & 3.19371 & -1.26108\end{array}$




\section{Supporting Information}

\section{Charts of ${ }^{1} \mathrm{H}^{-}$and ${ }^{13} \mathrm{C}-\mathrm{NMR}$ Spectra}

The $\mathrm{dr}$ values were determined by the signals inside the red frames.

$4 a$

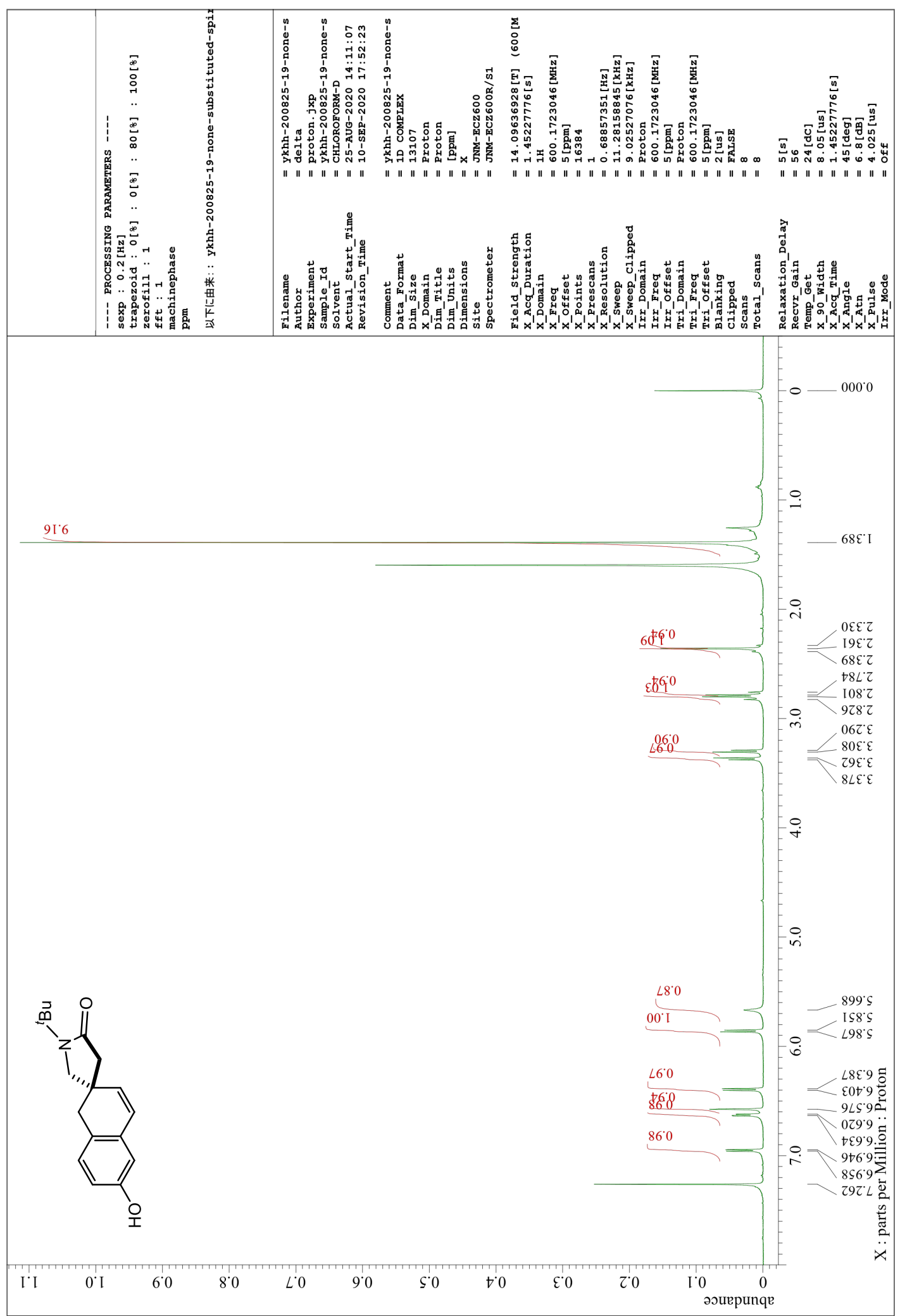




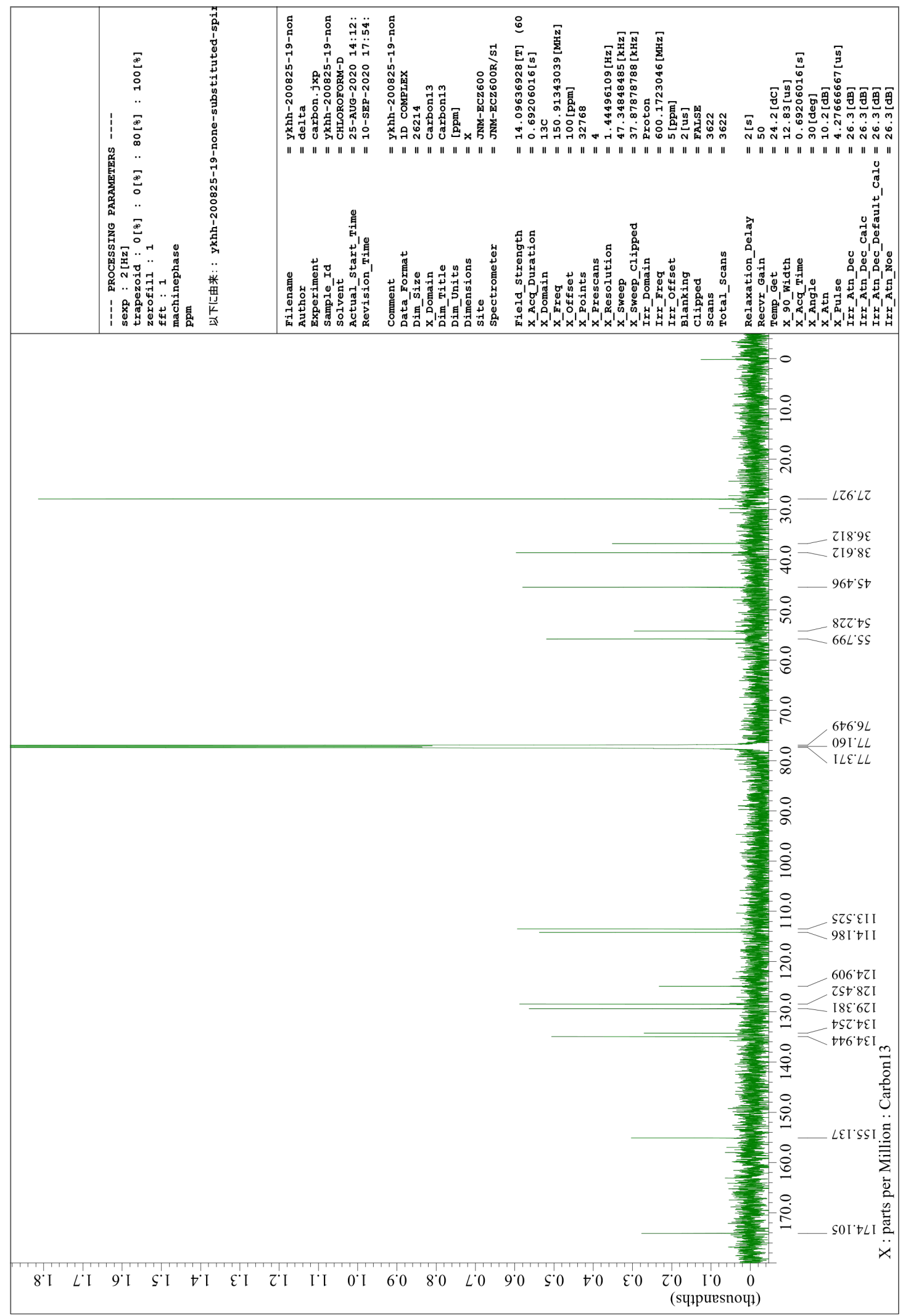


$4 b$

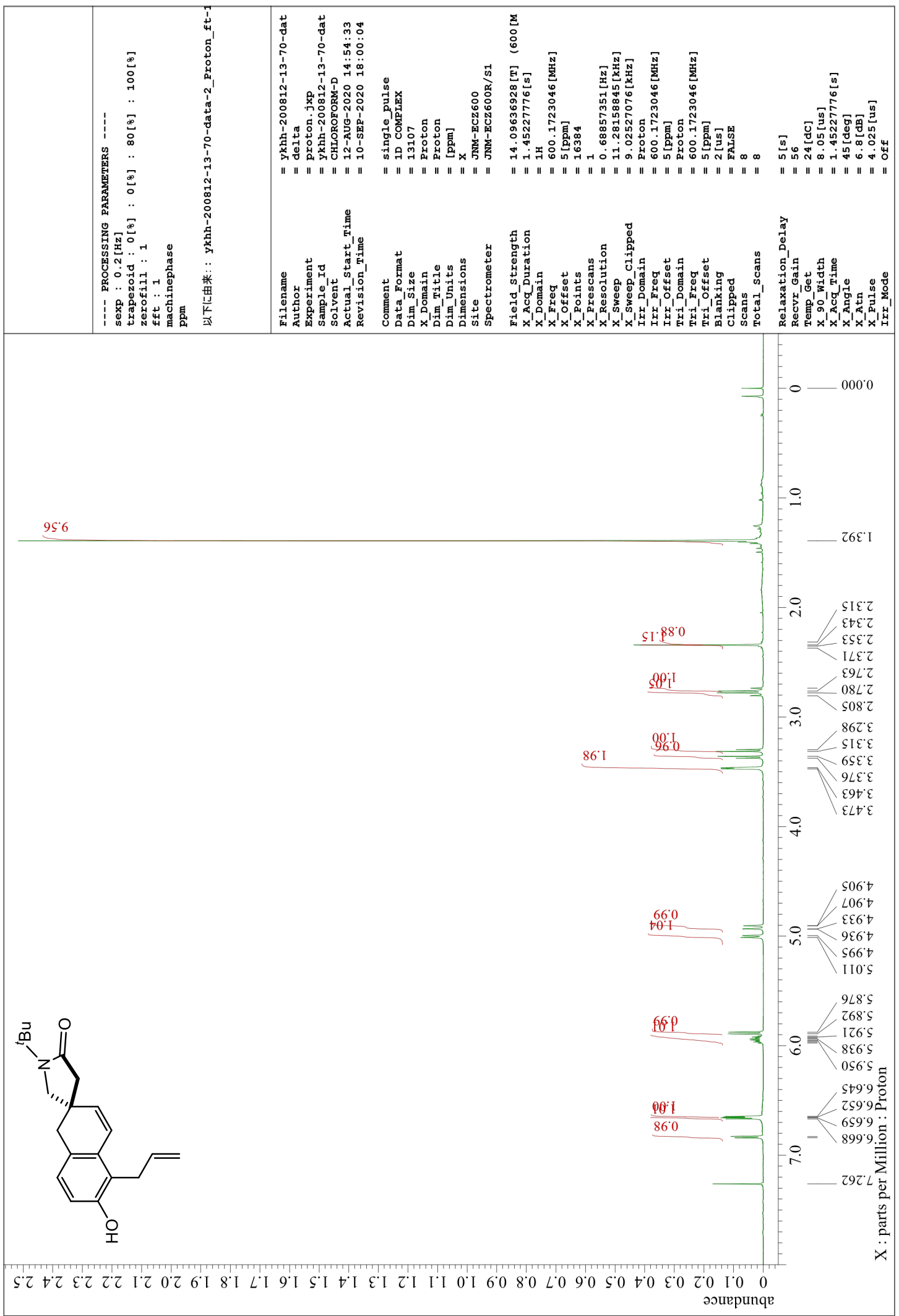




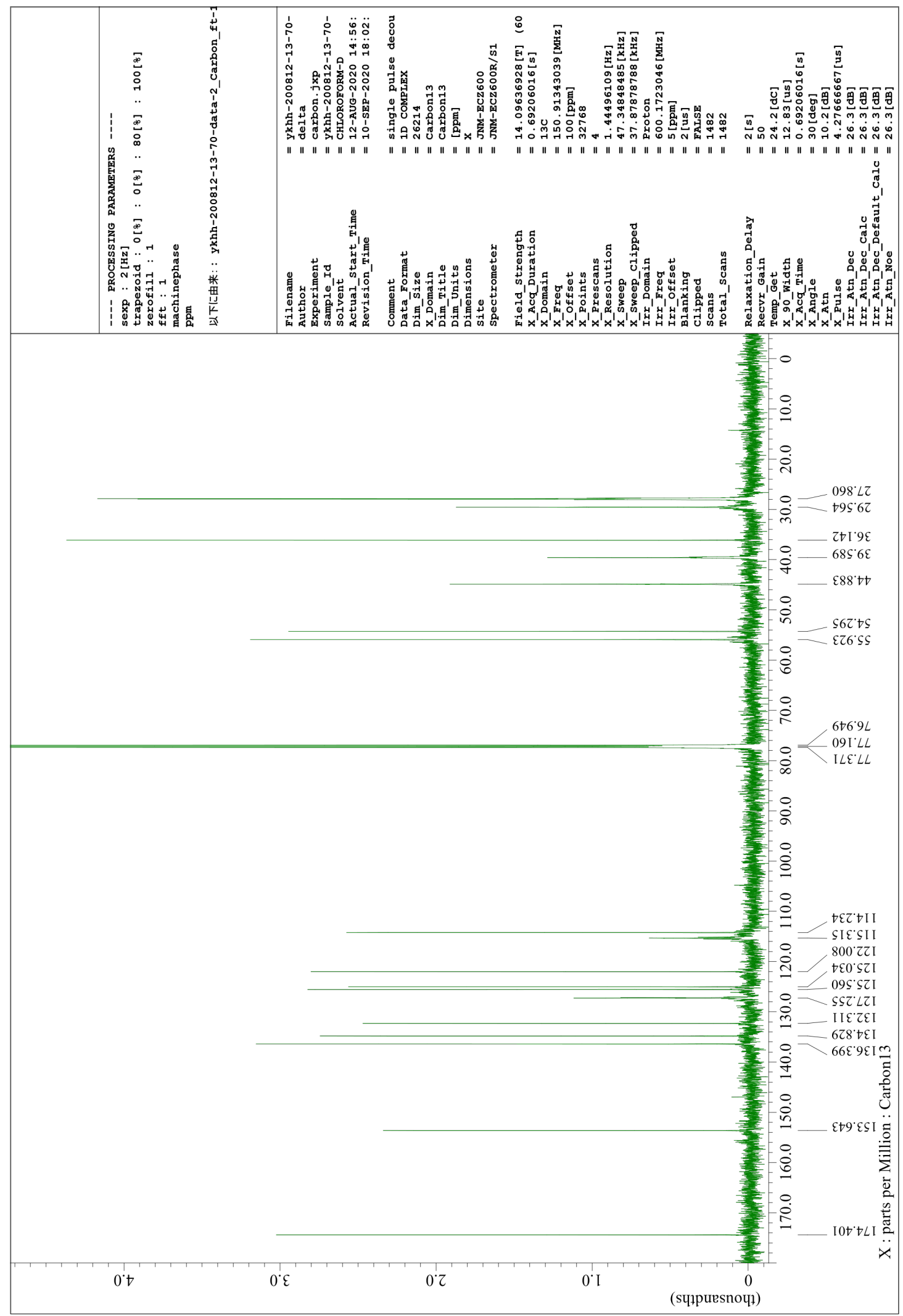


$4 c$

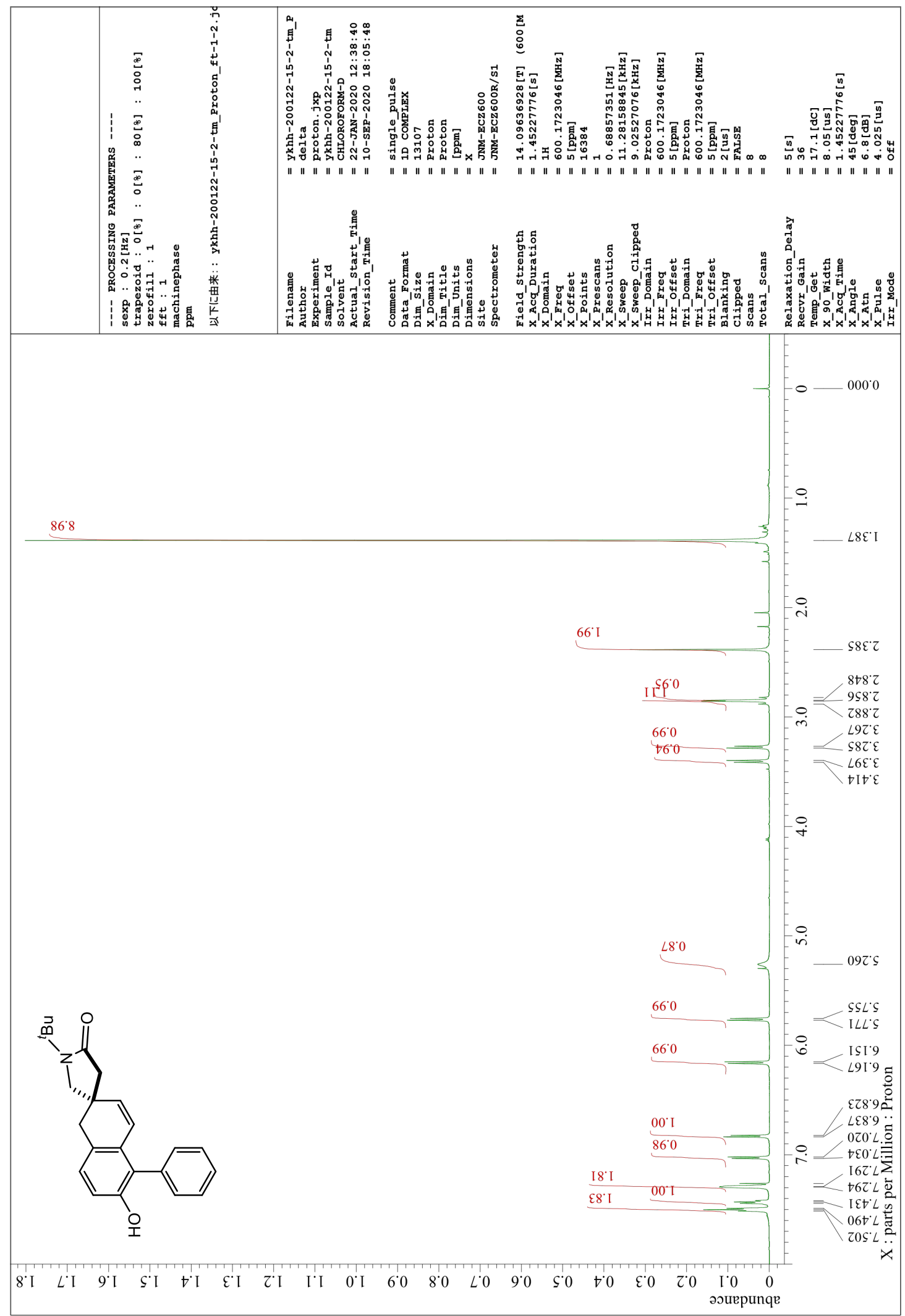




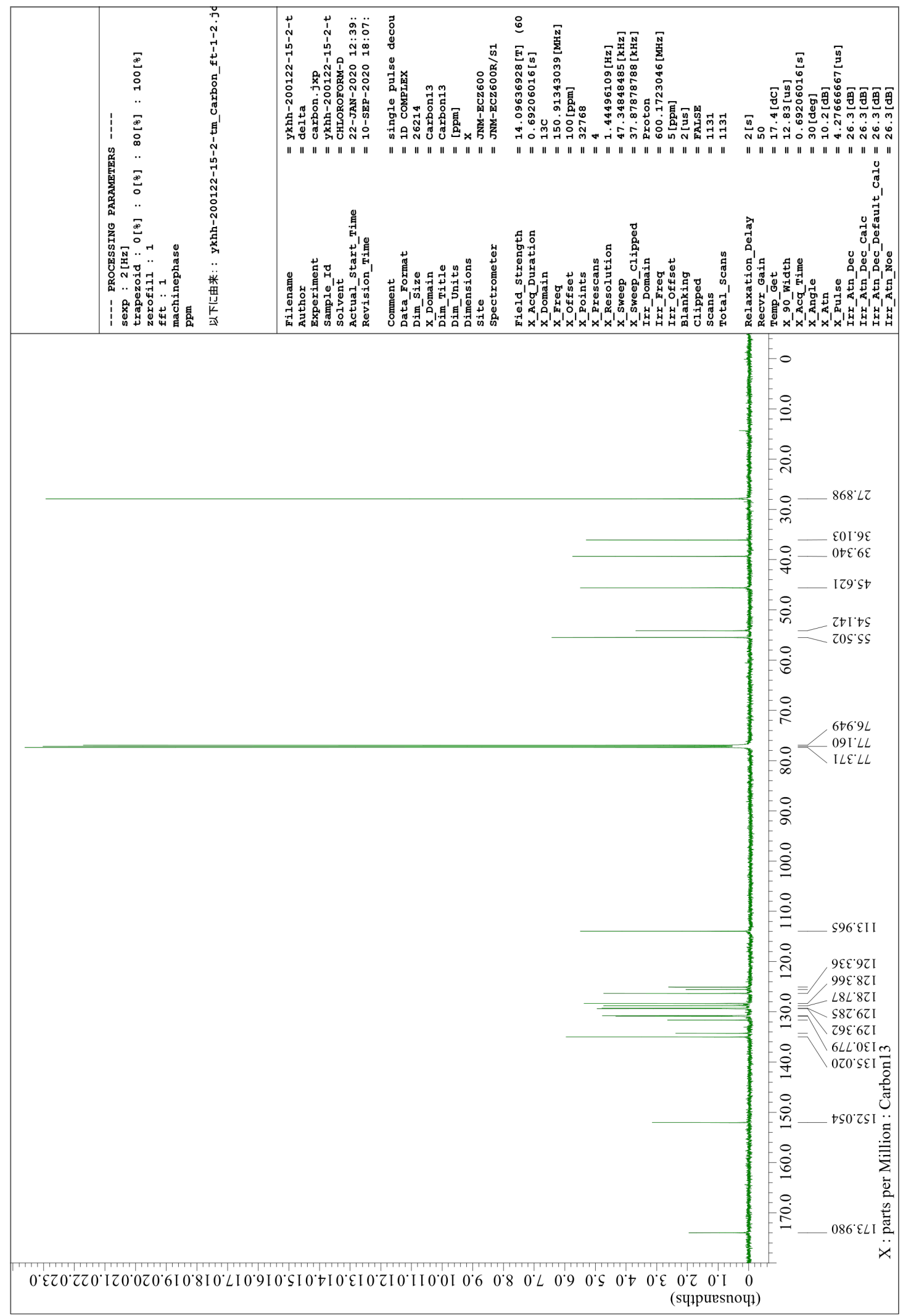


Supporting Information

$4 d$

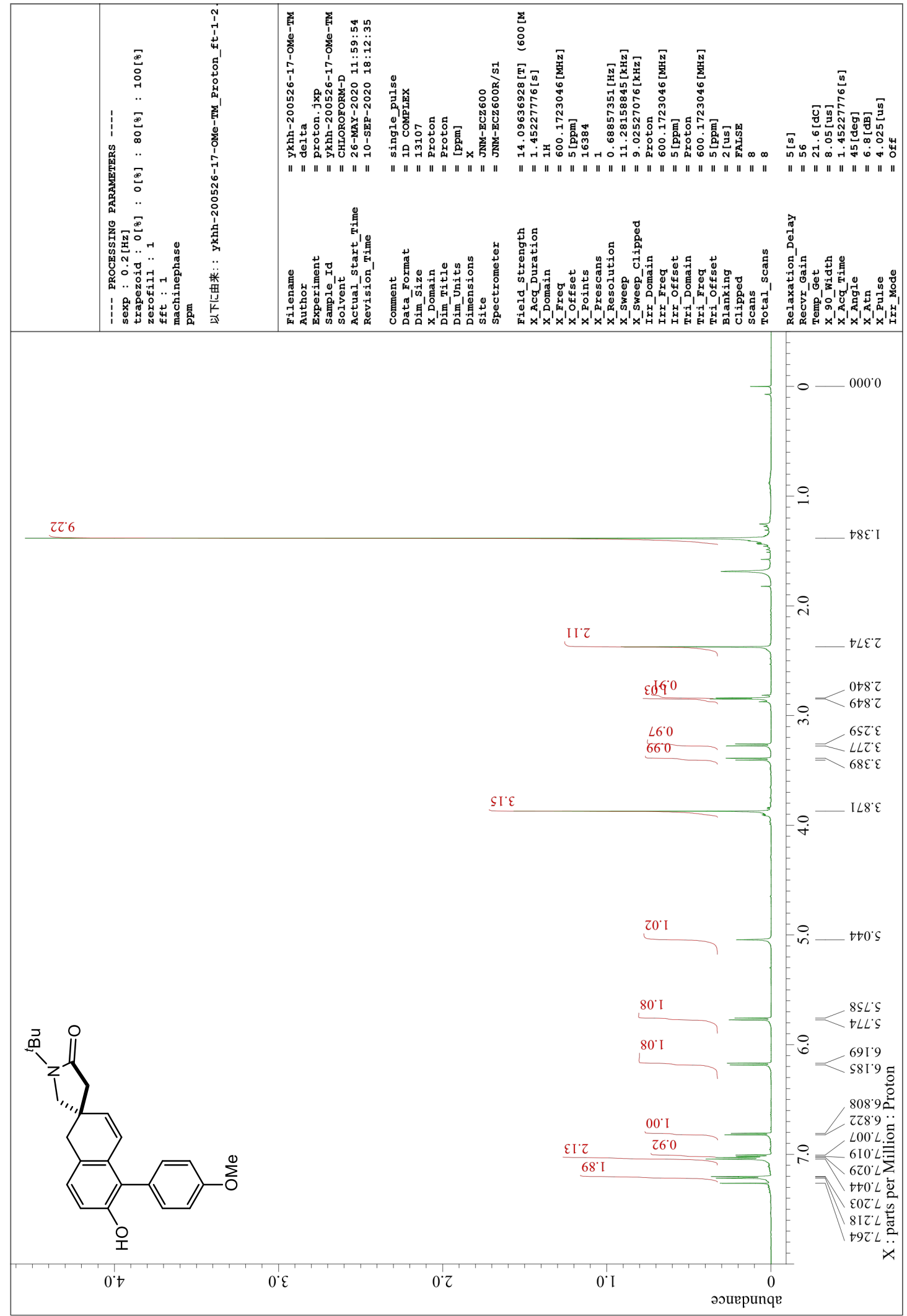




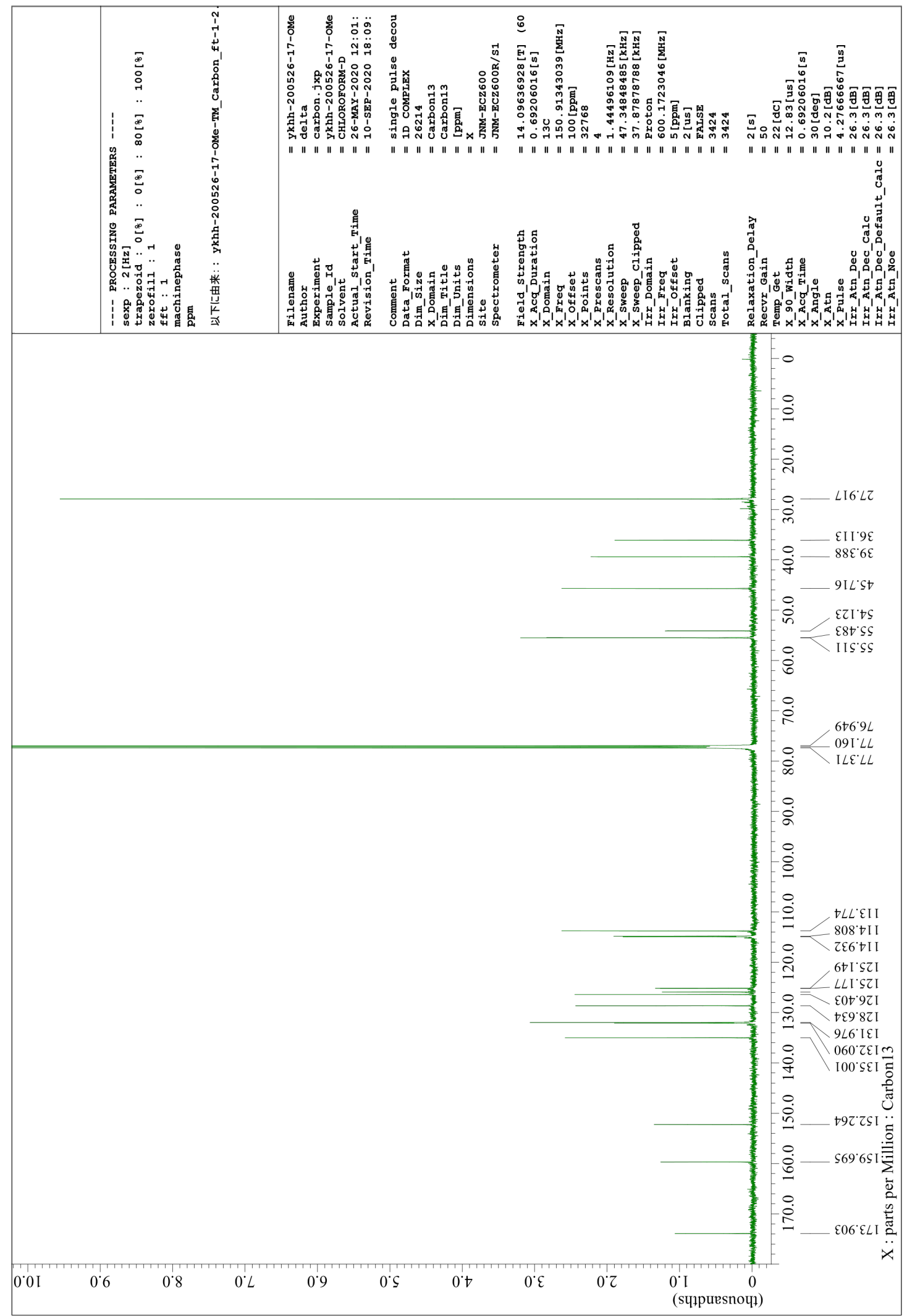


$4 e$

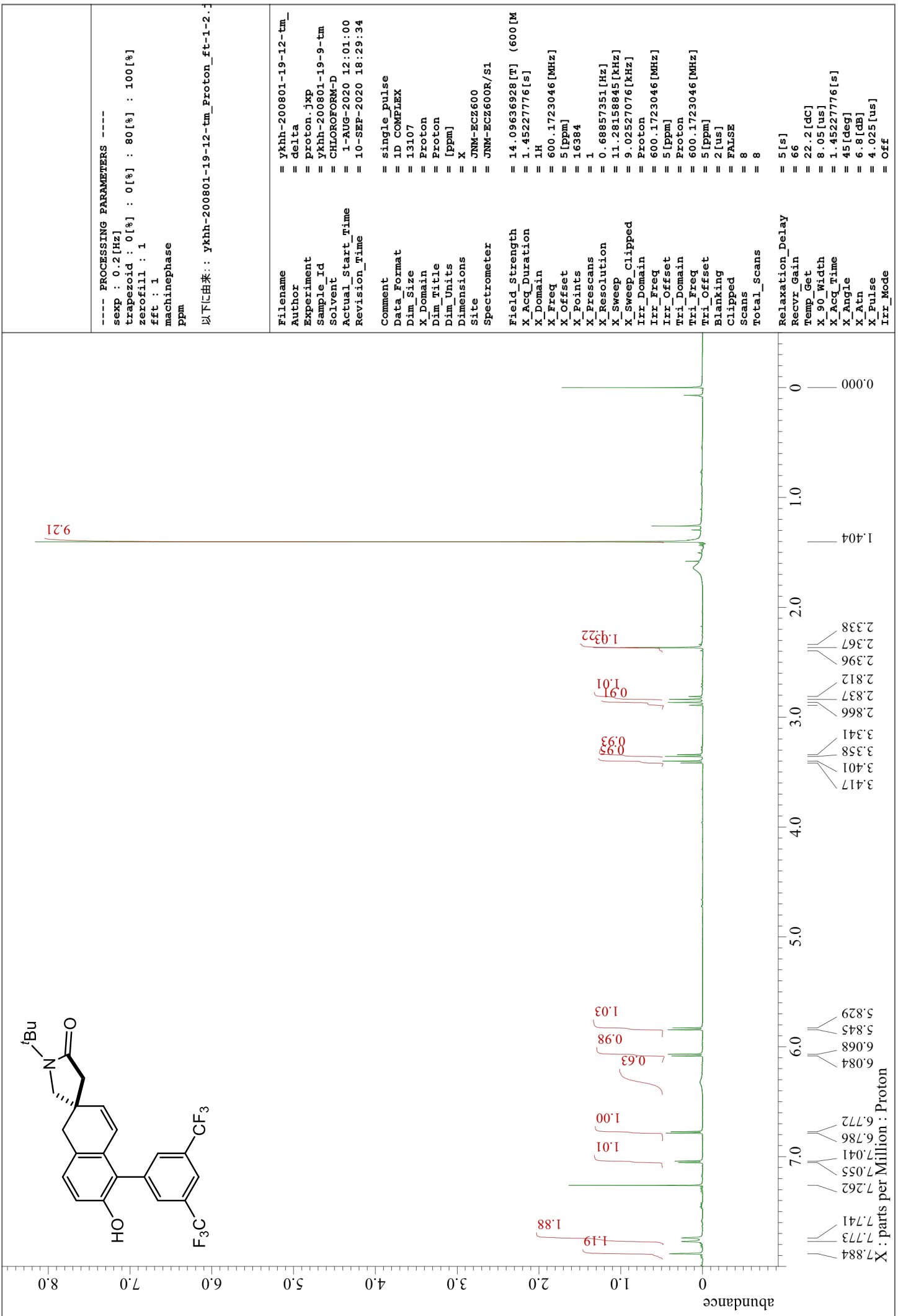


Supporting Information

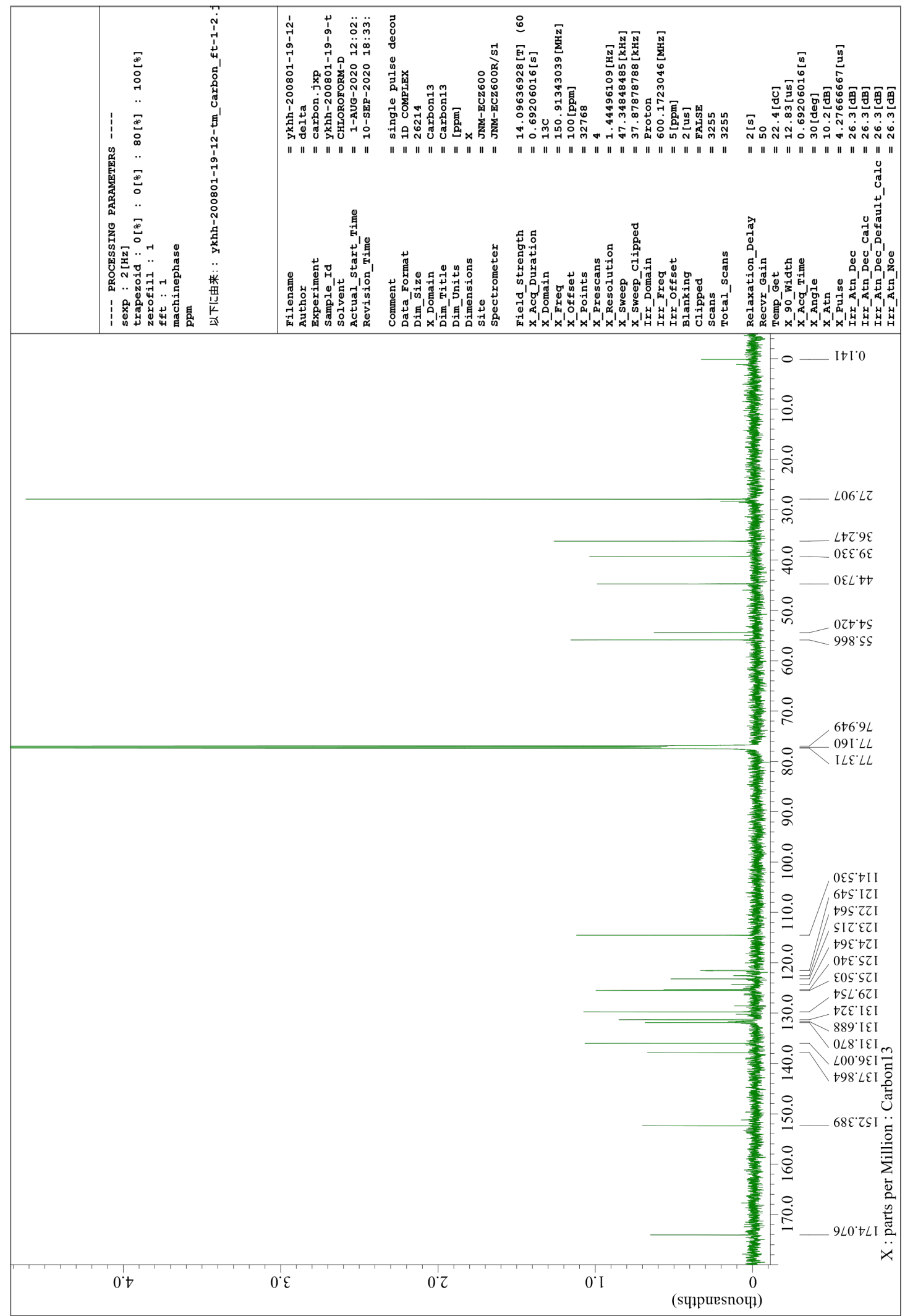




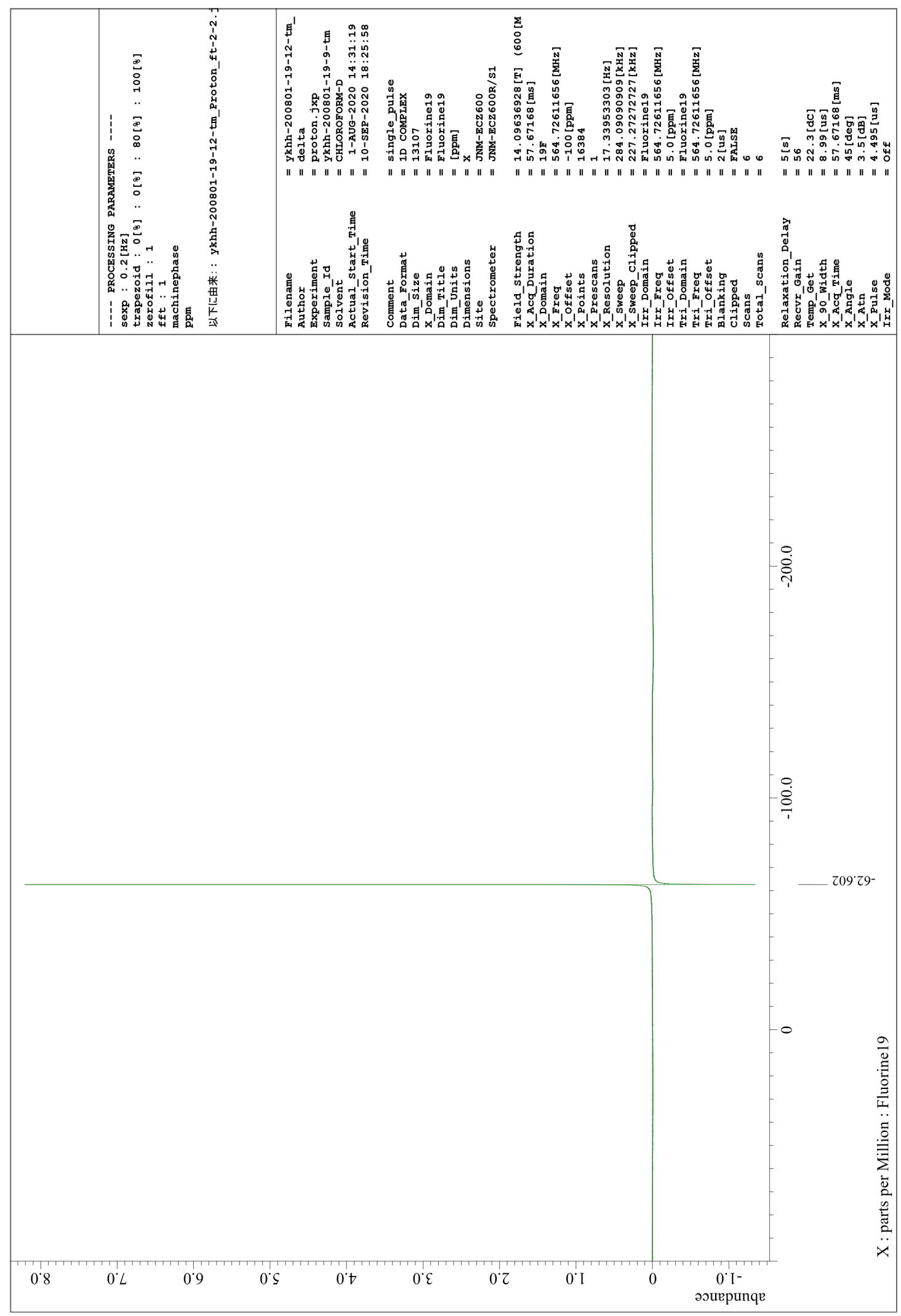


$4 f$

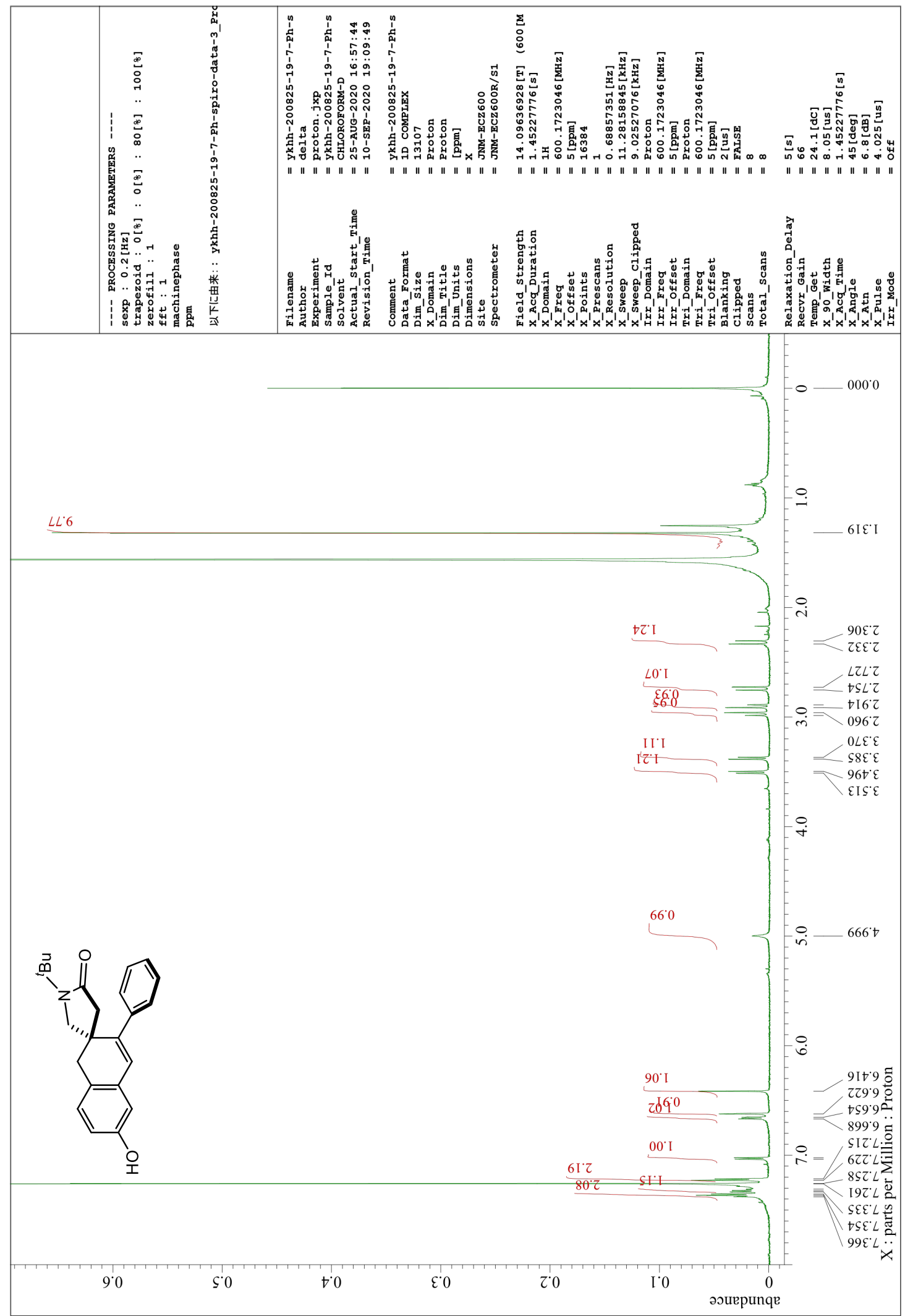




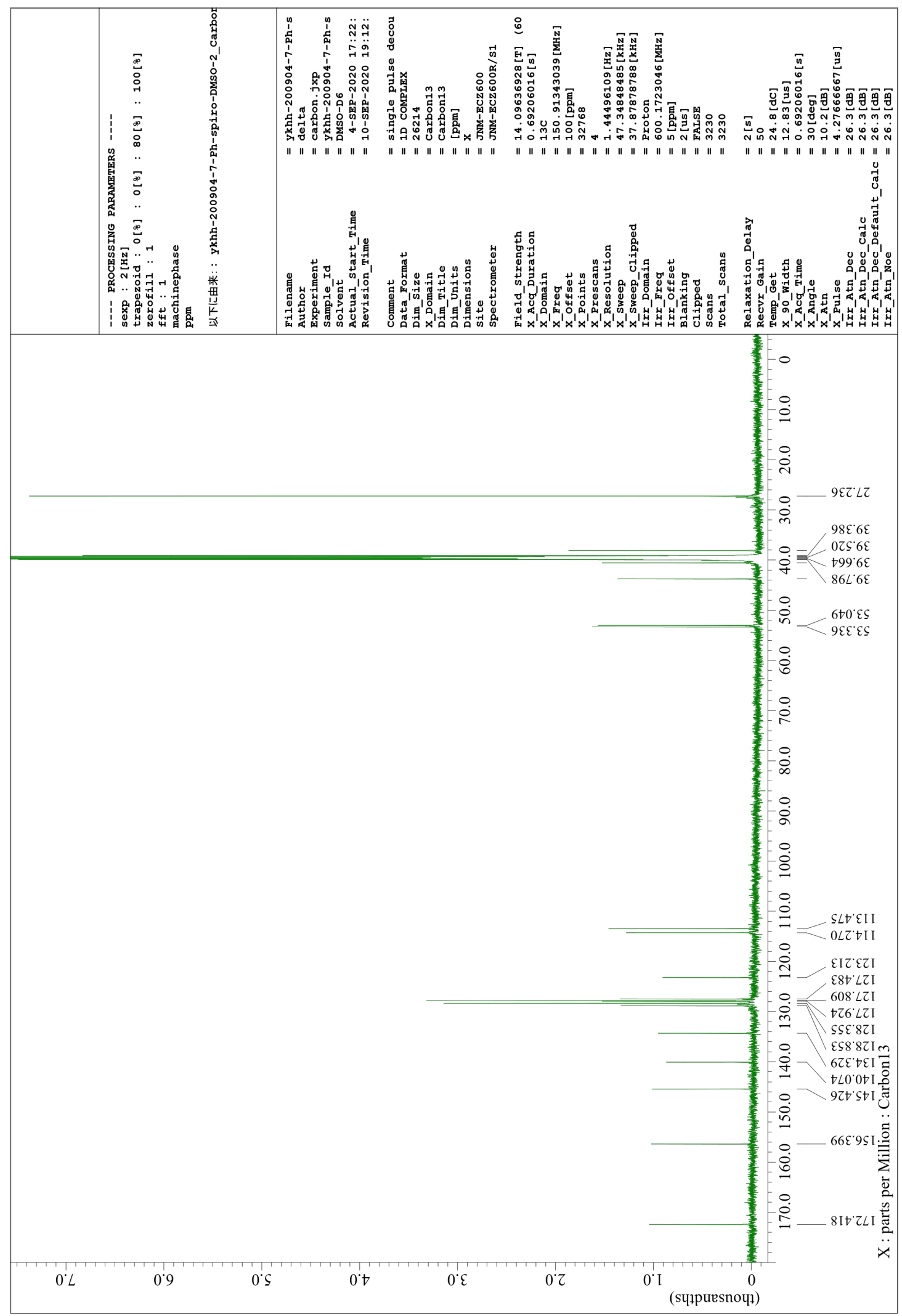


Supporting Information

$4 g$

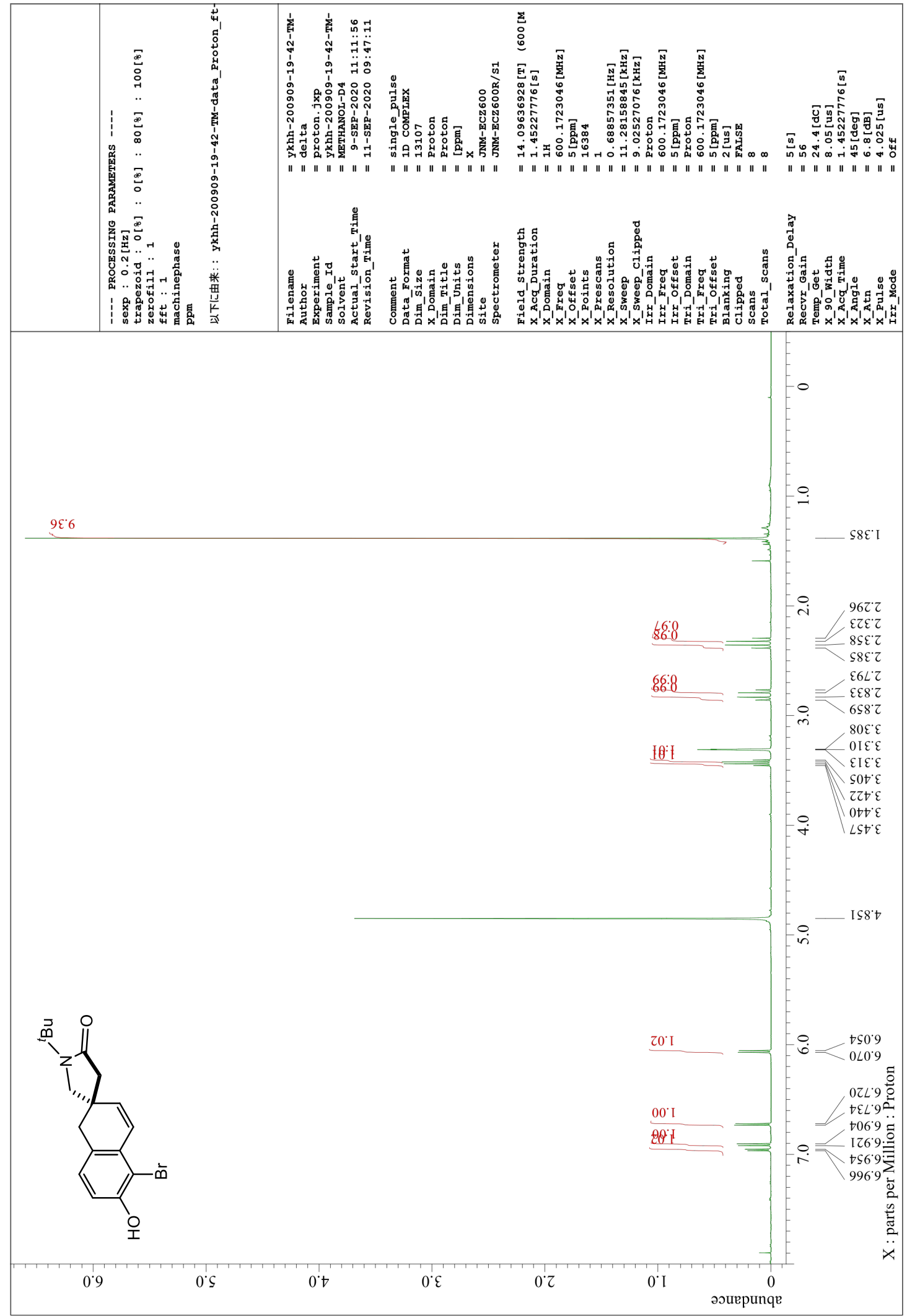




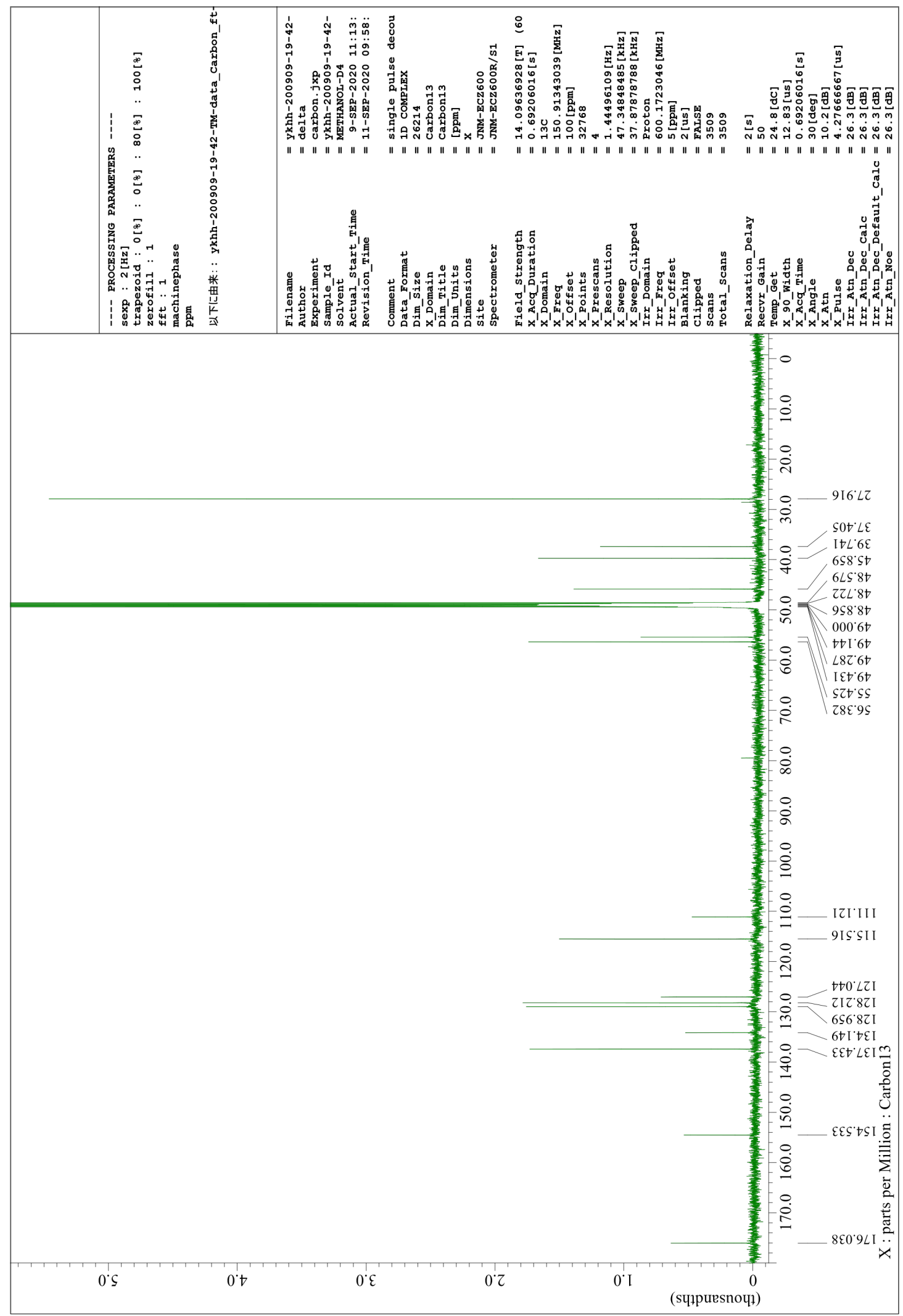


$4 \mathrm{~h}$

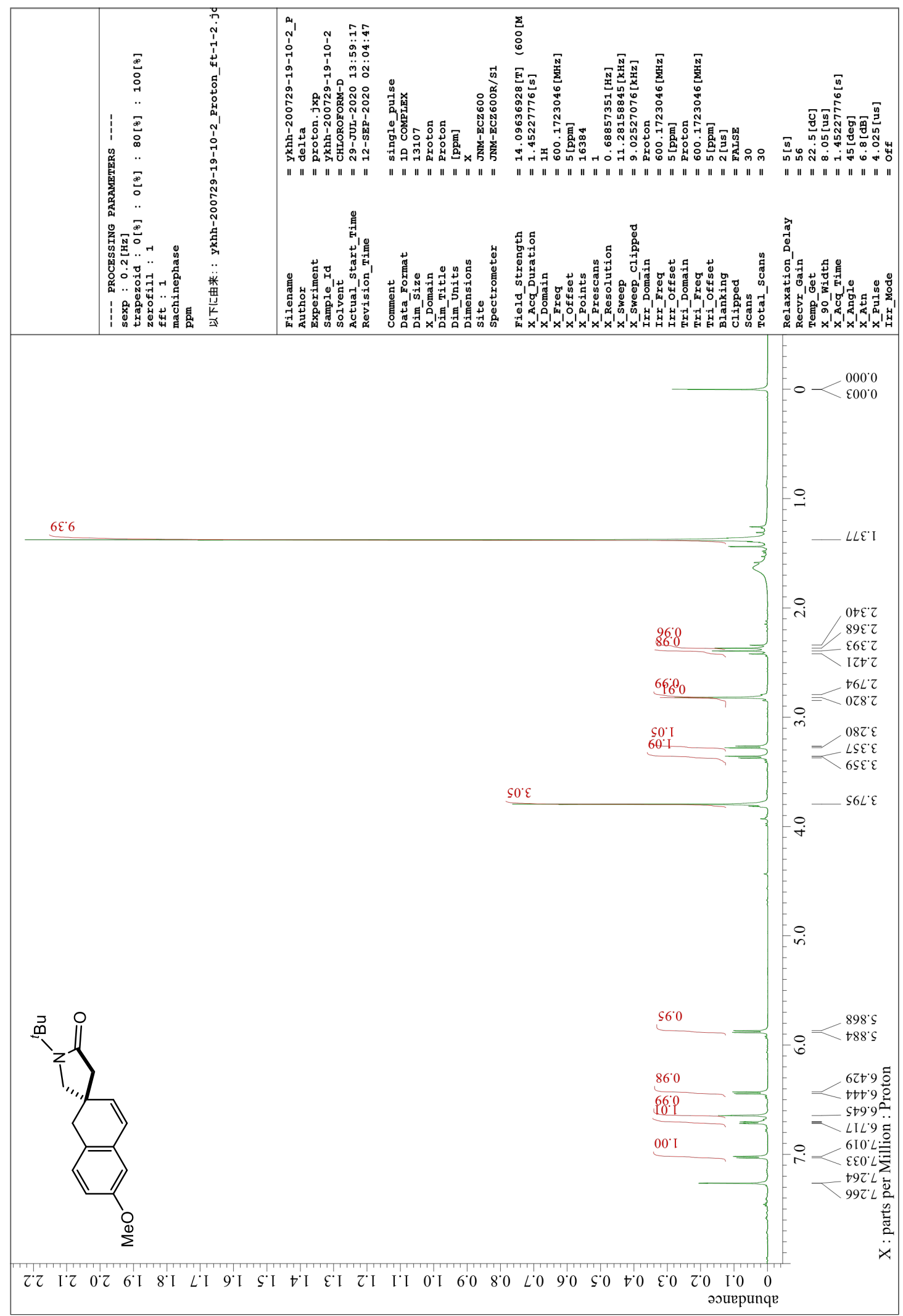




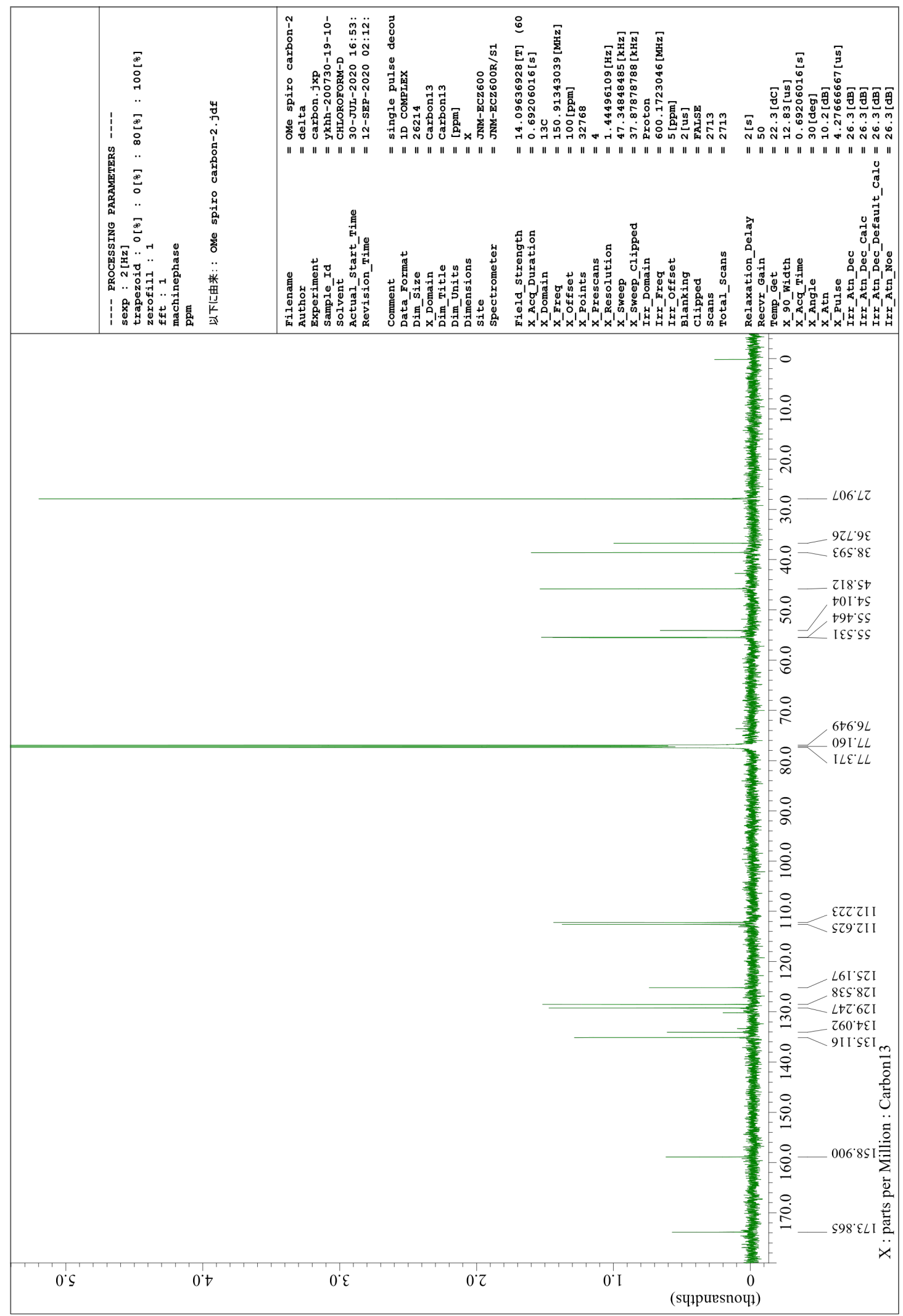


$4 \mathbf{i}$

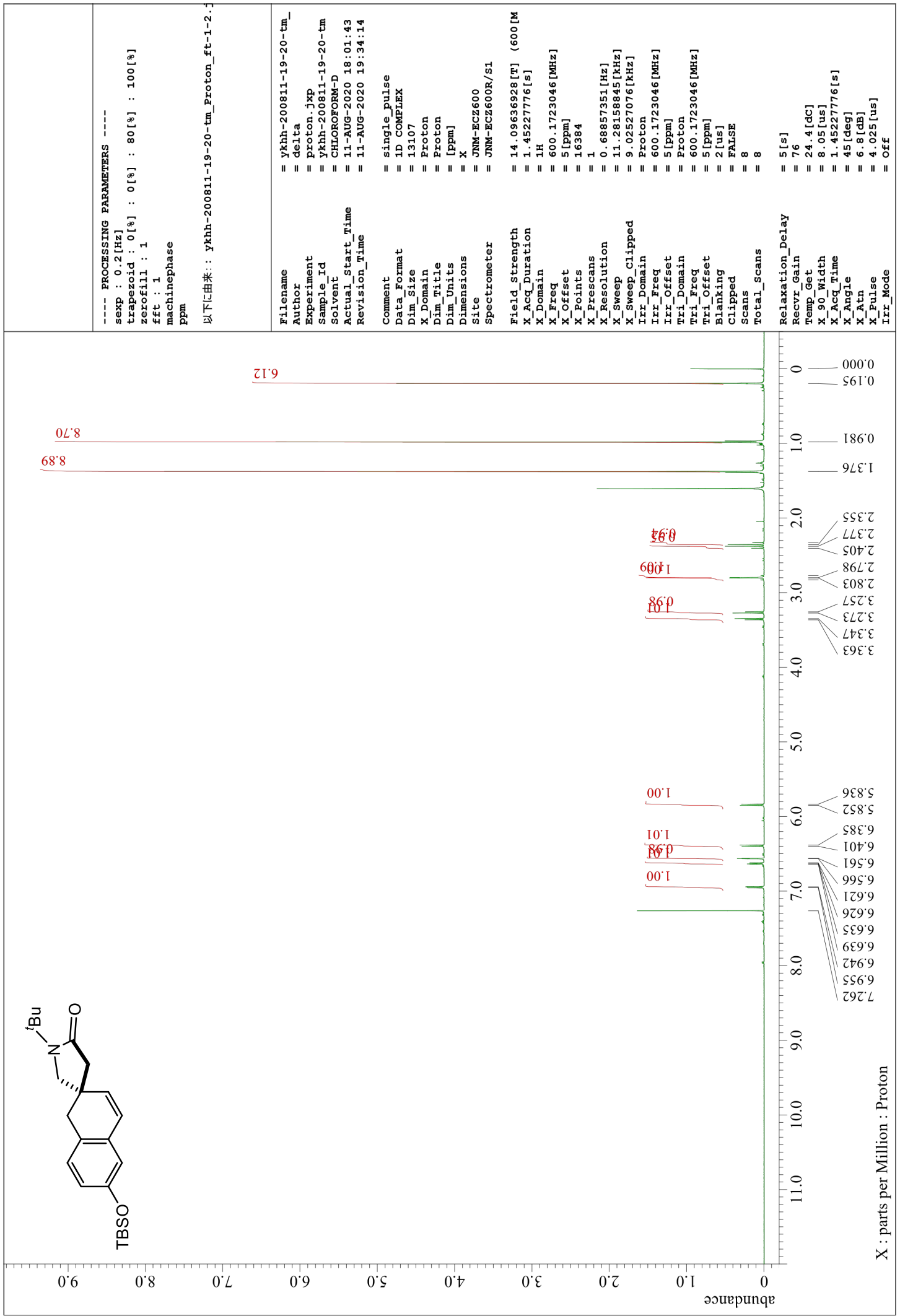




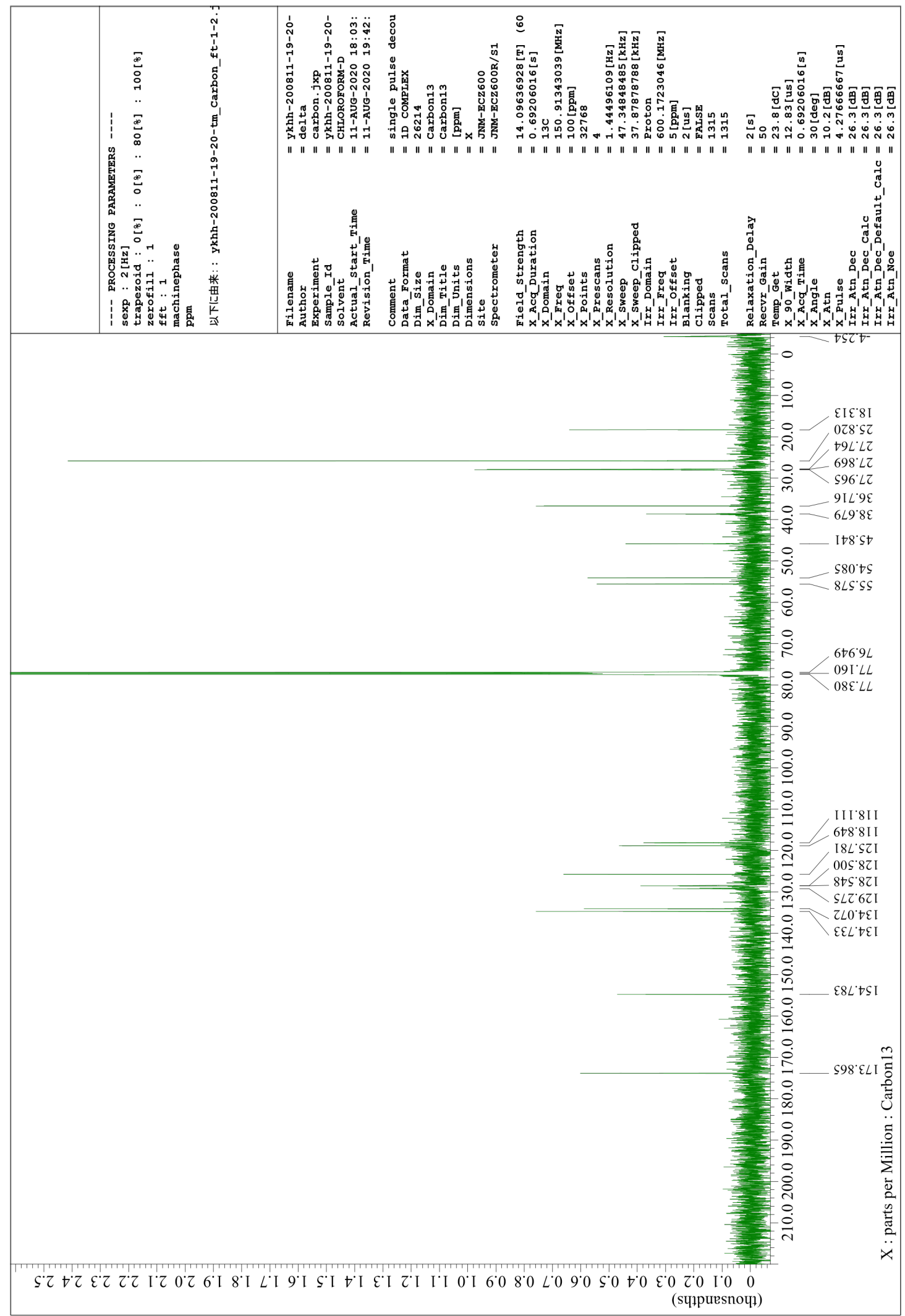


$5 a$

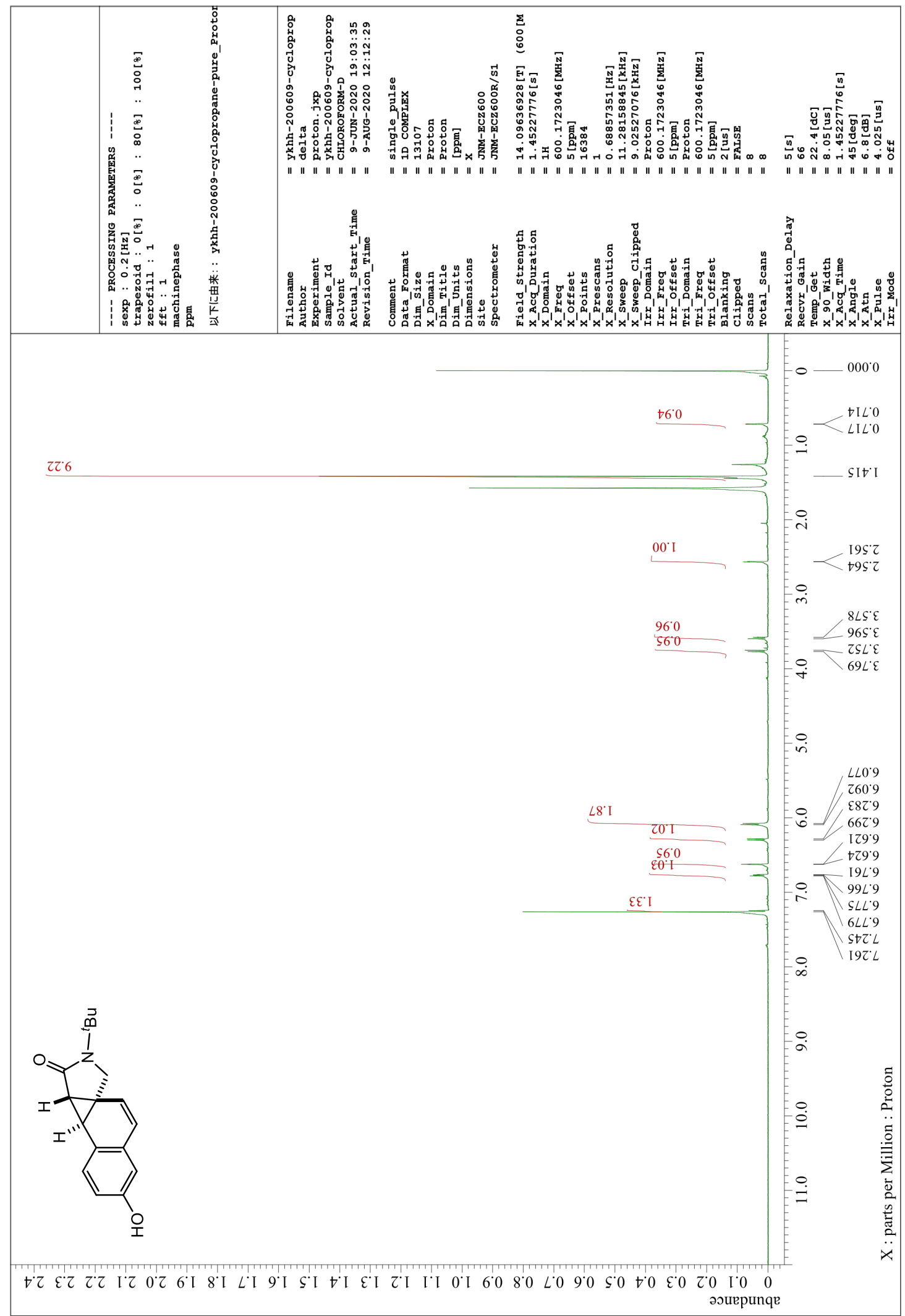




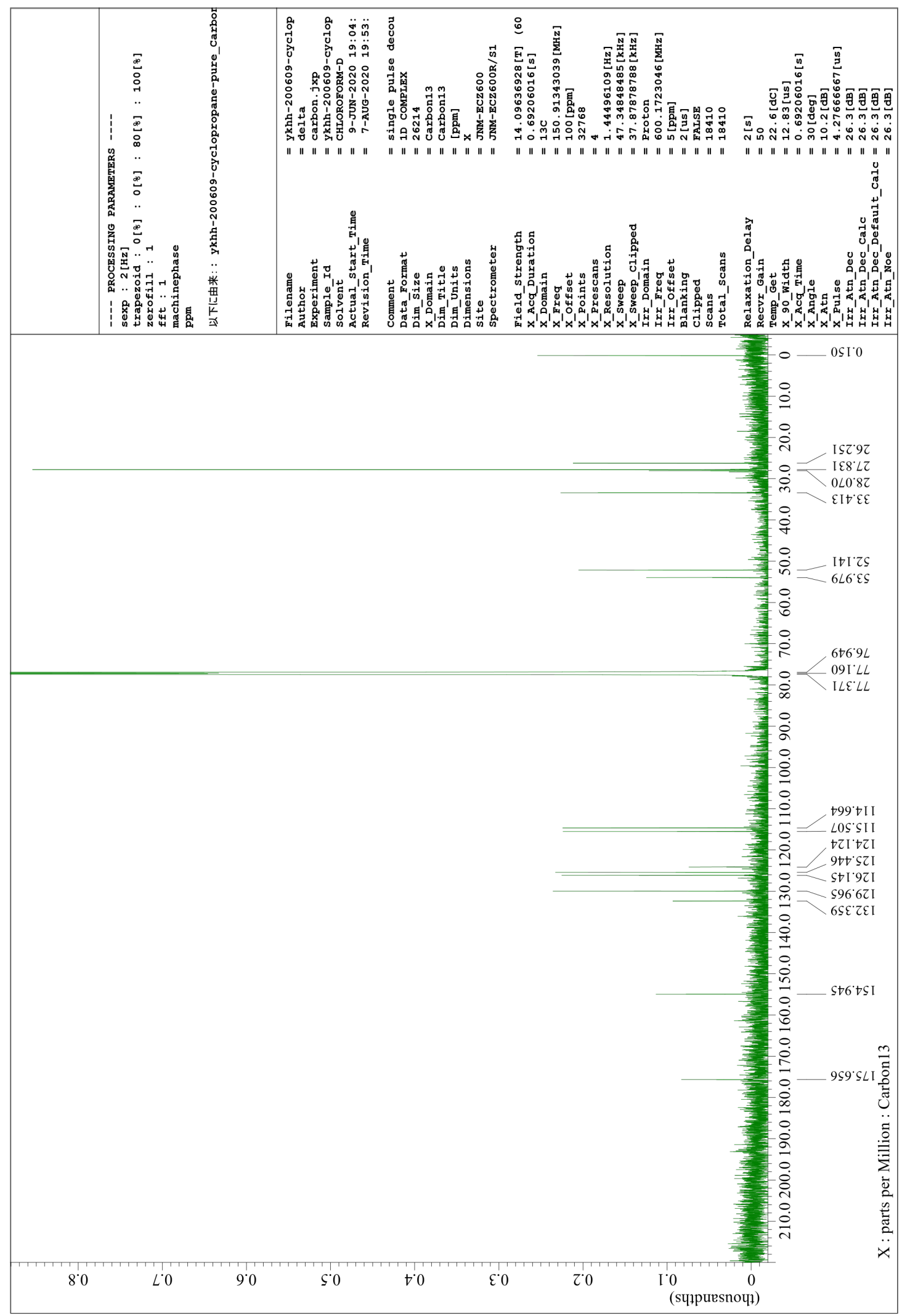


$6 a$

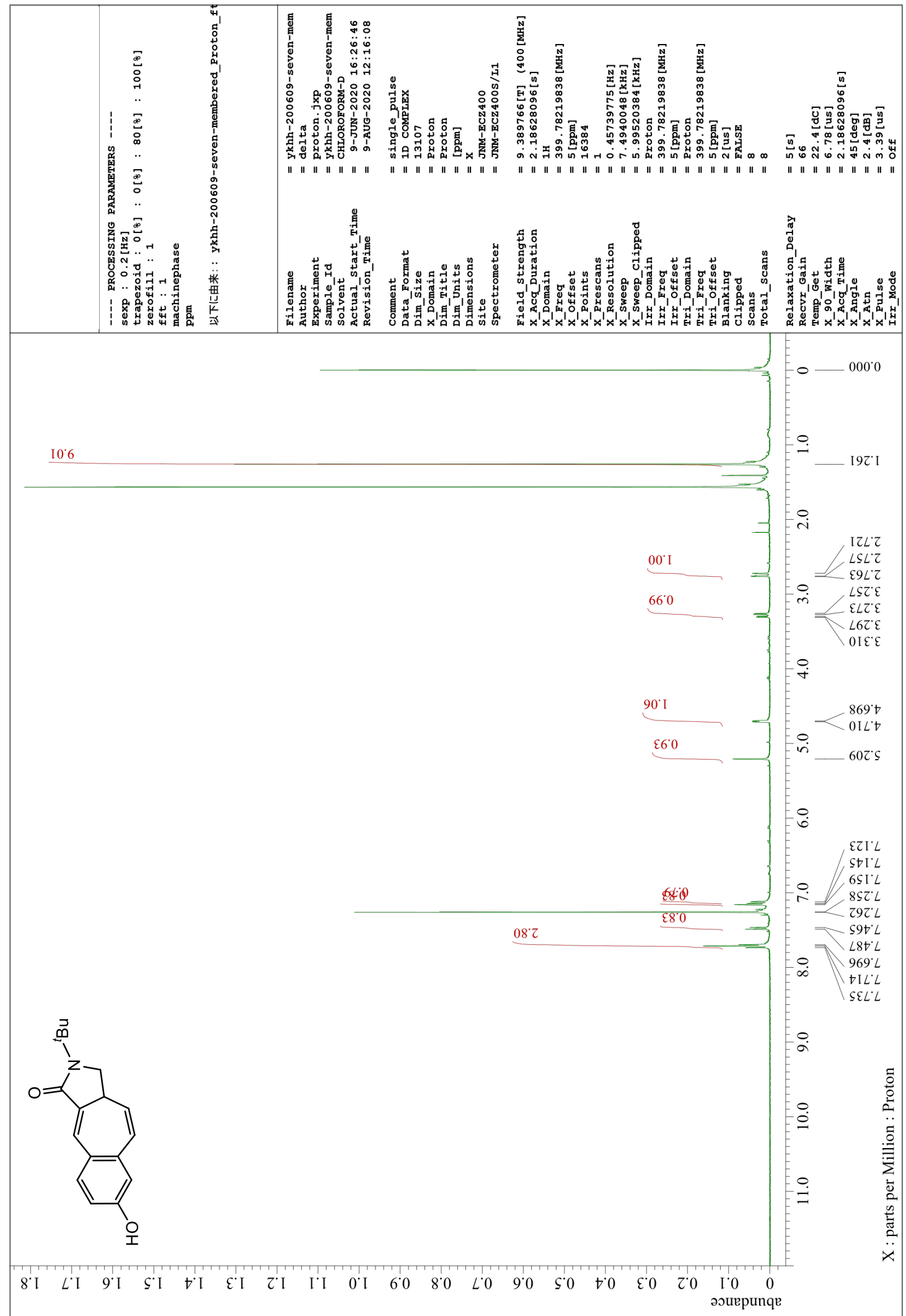




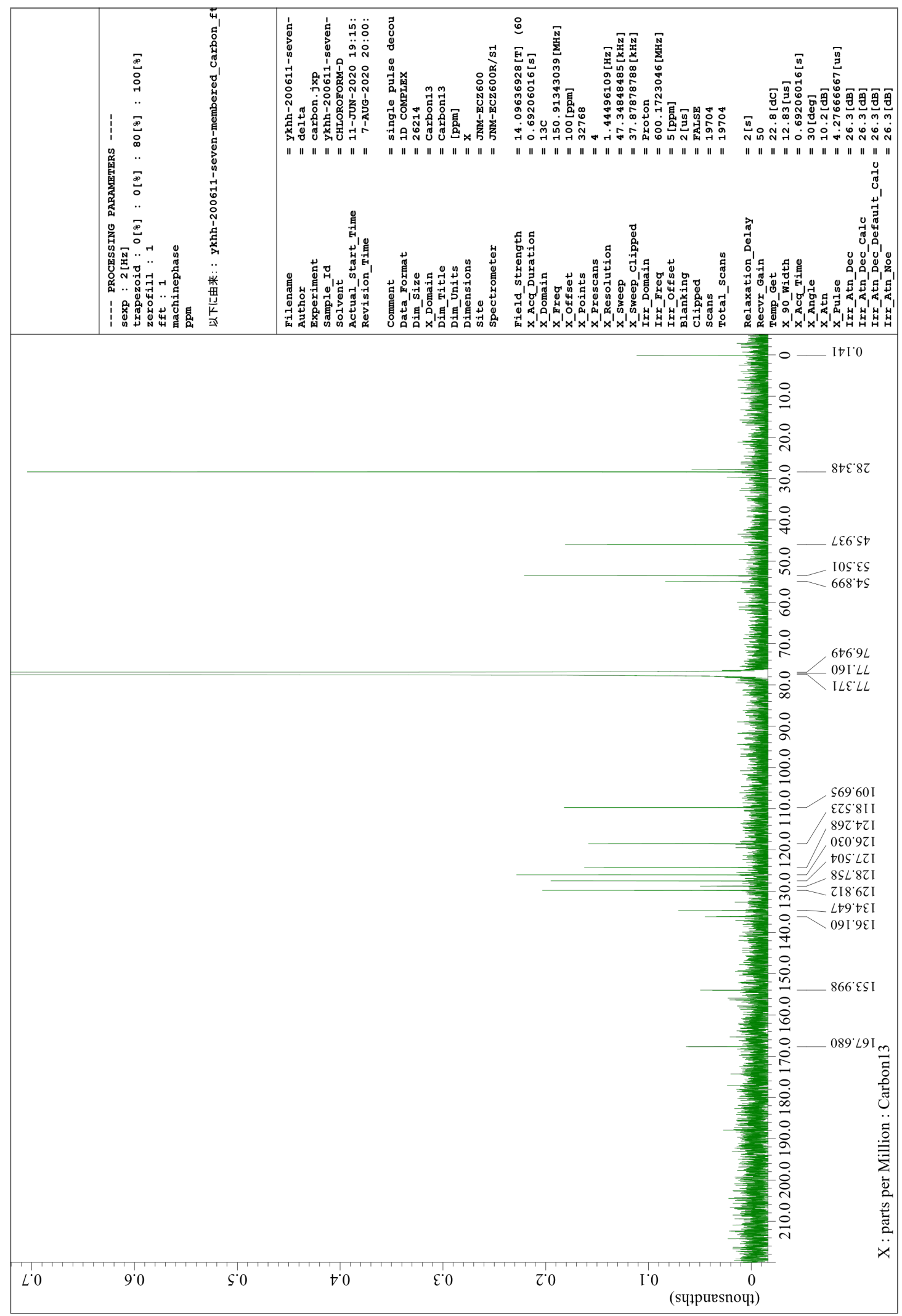


Supporting Information

$7 \mathrm{c}$

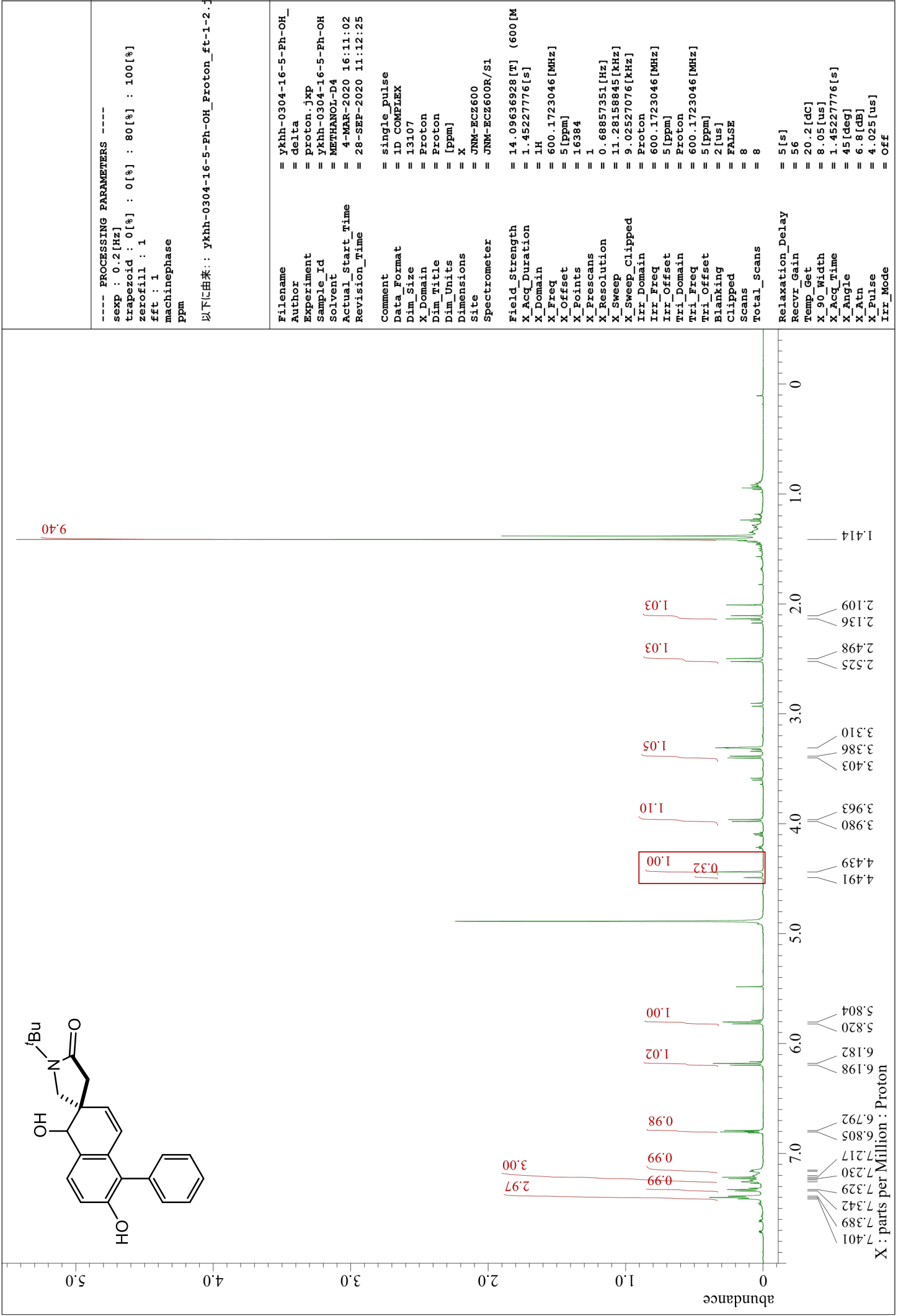




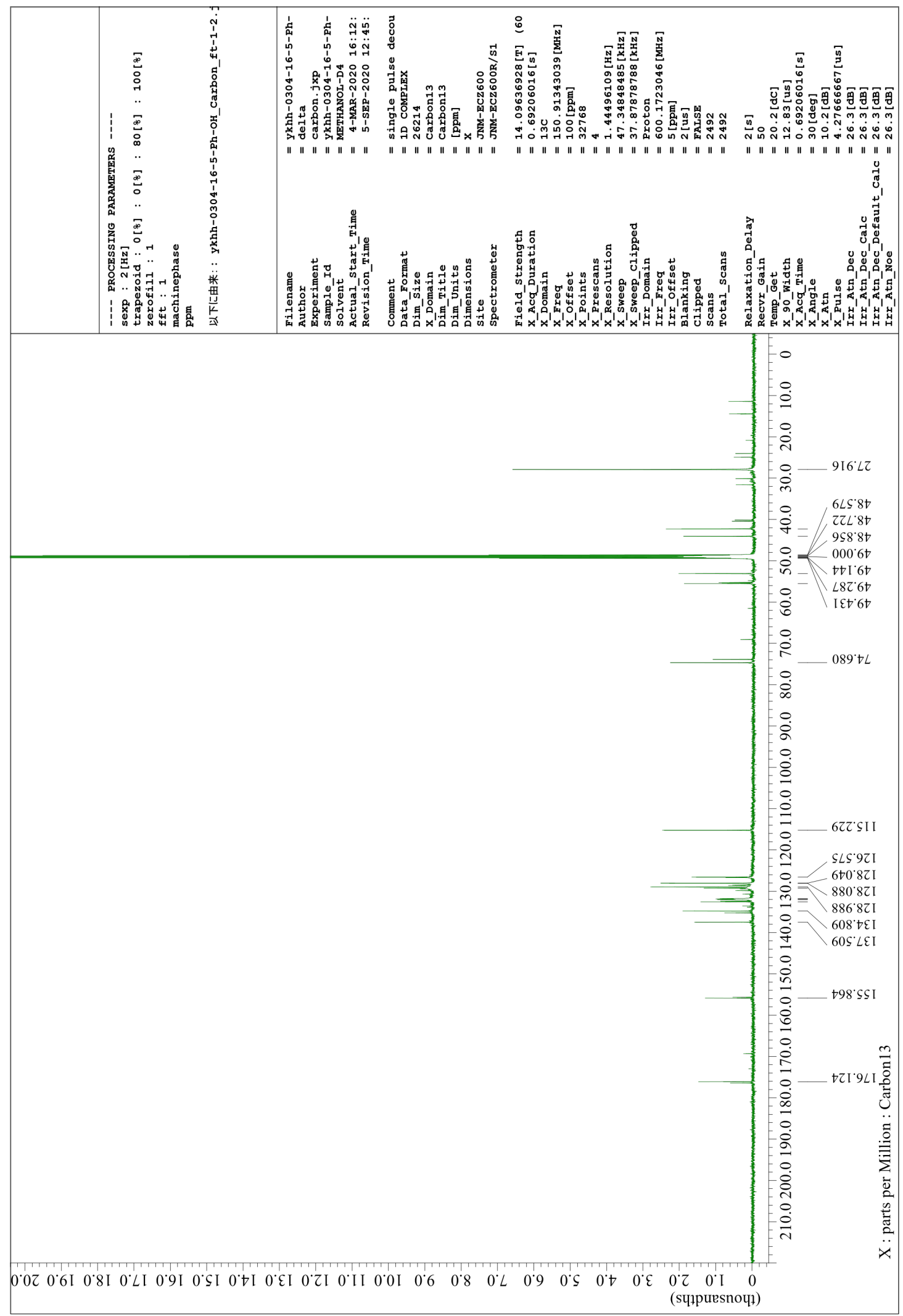


Supporting Information

$8 c$

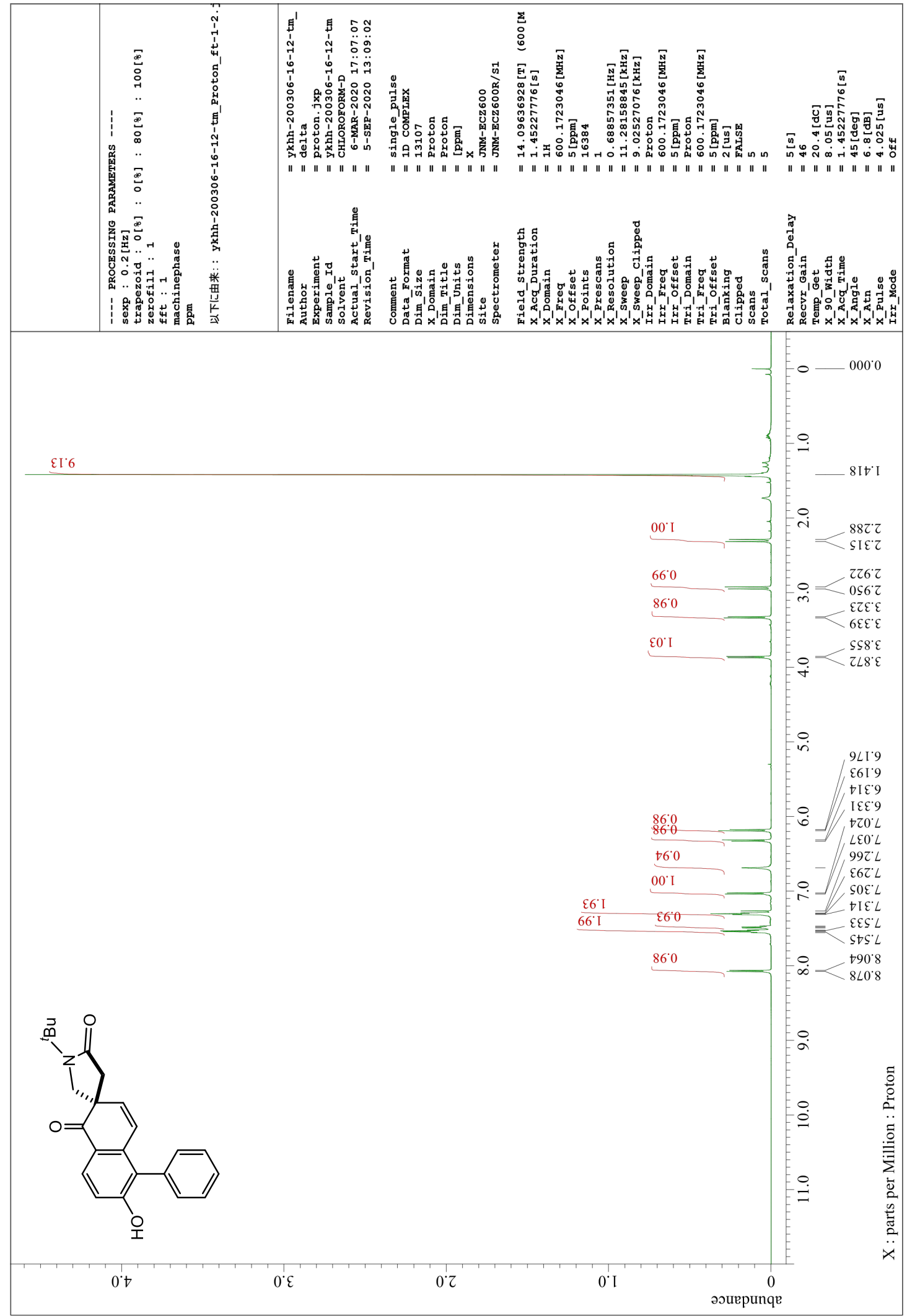




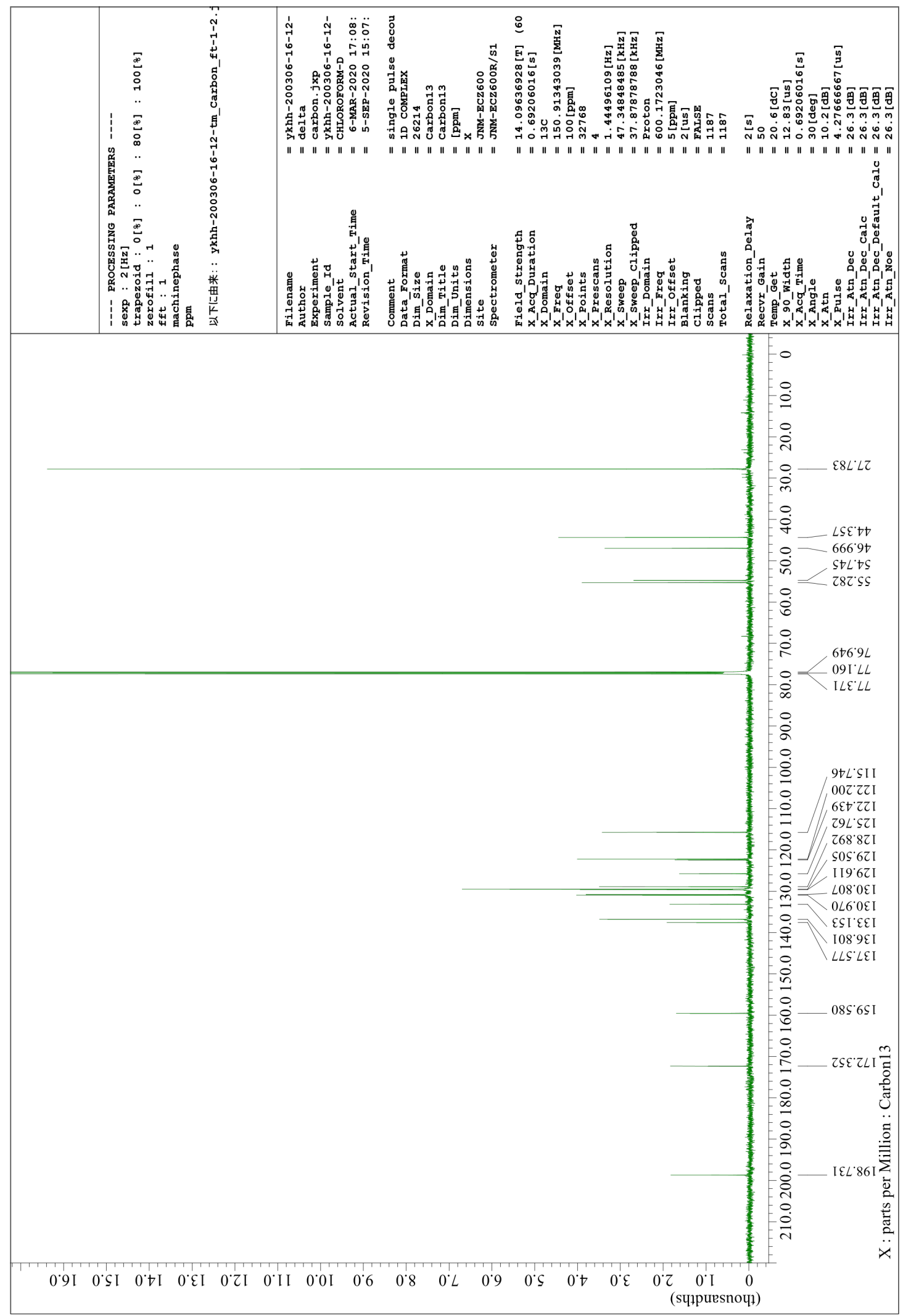


Supporting Information

$9 a$

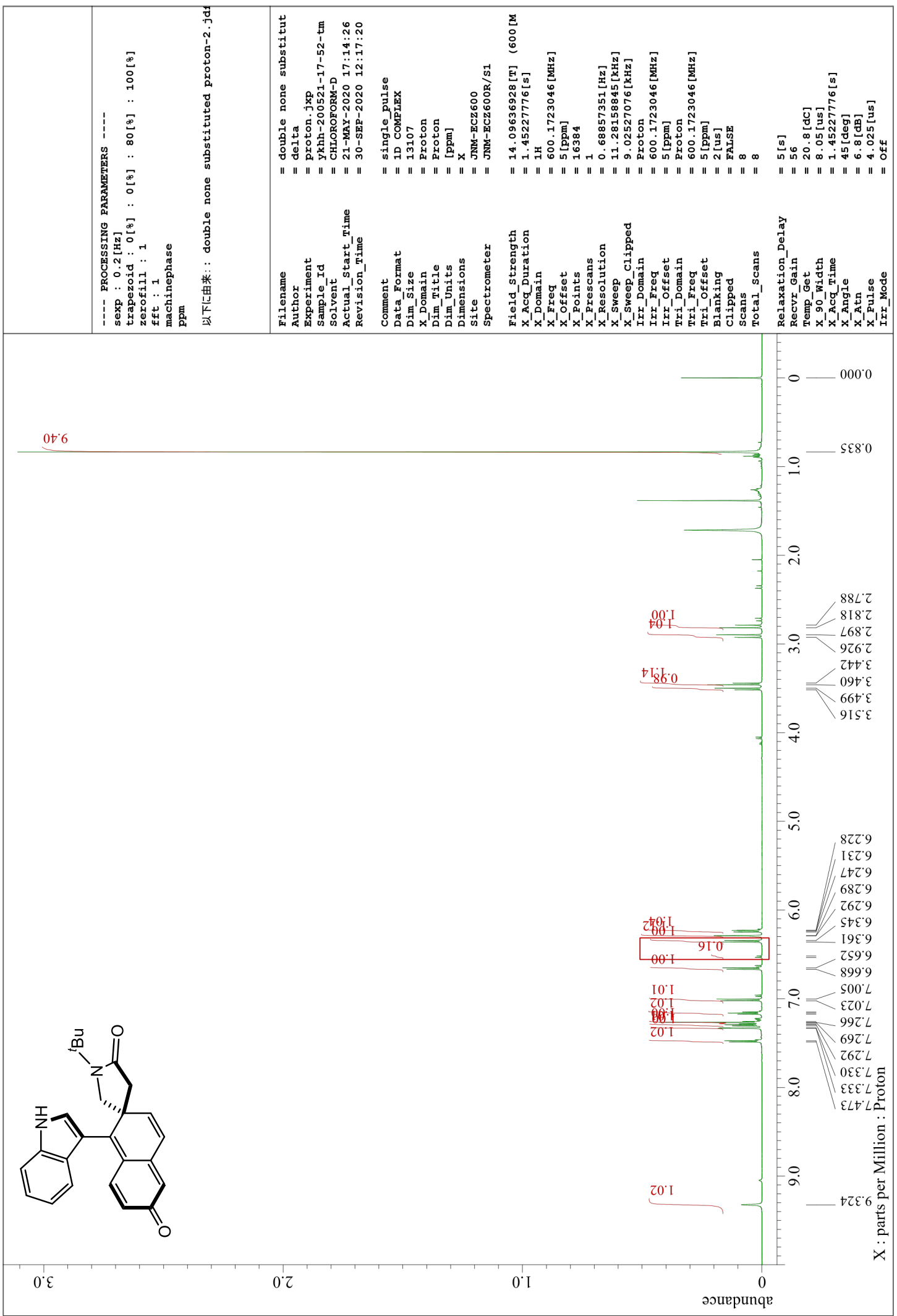




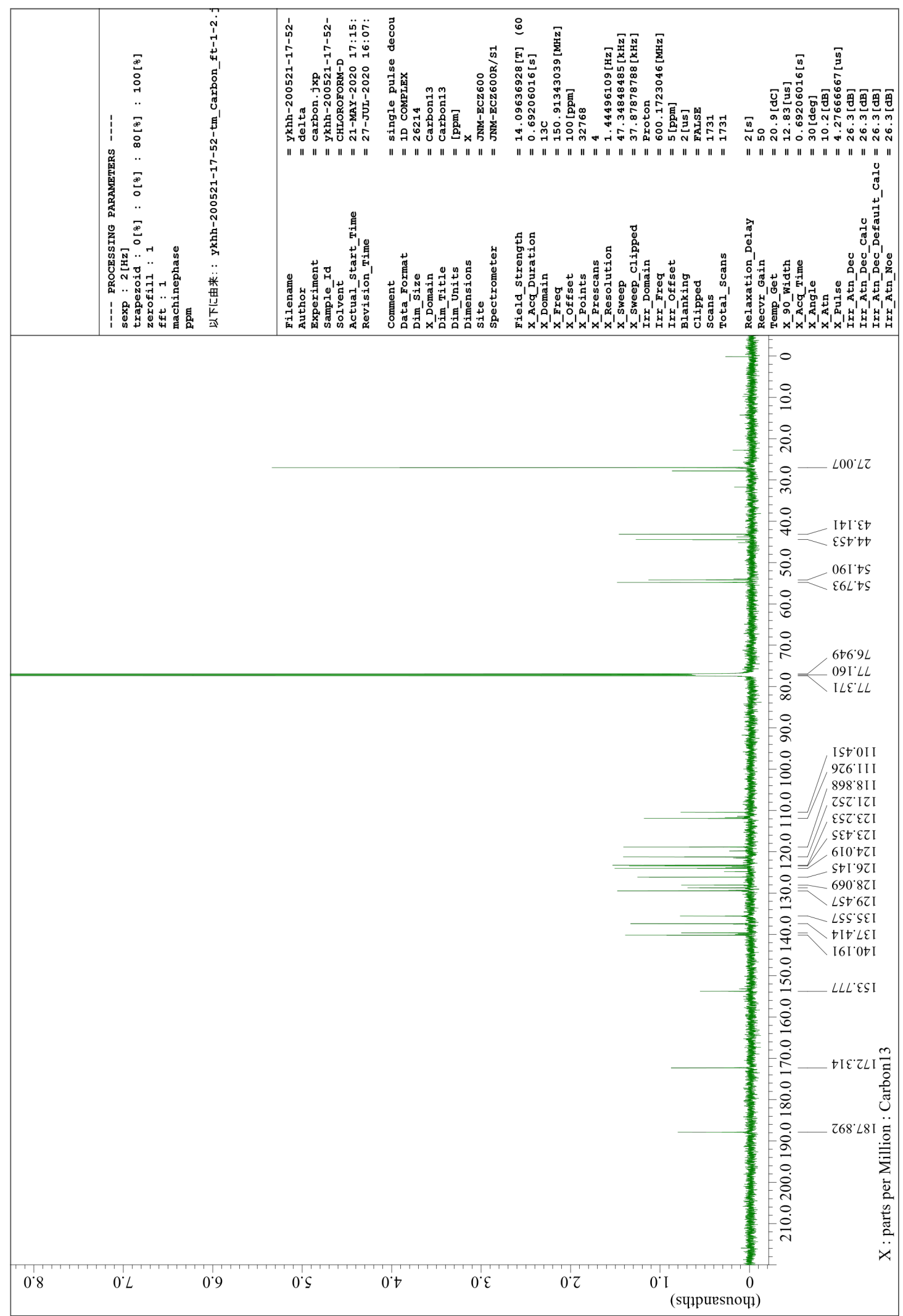


$9 b$

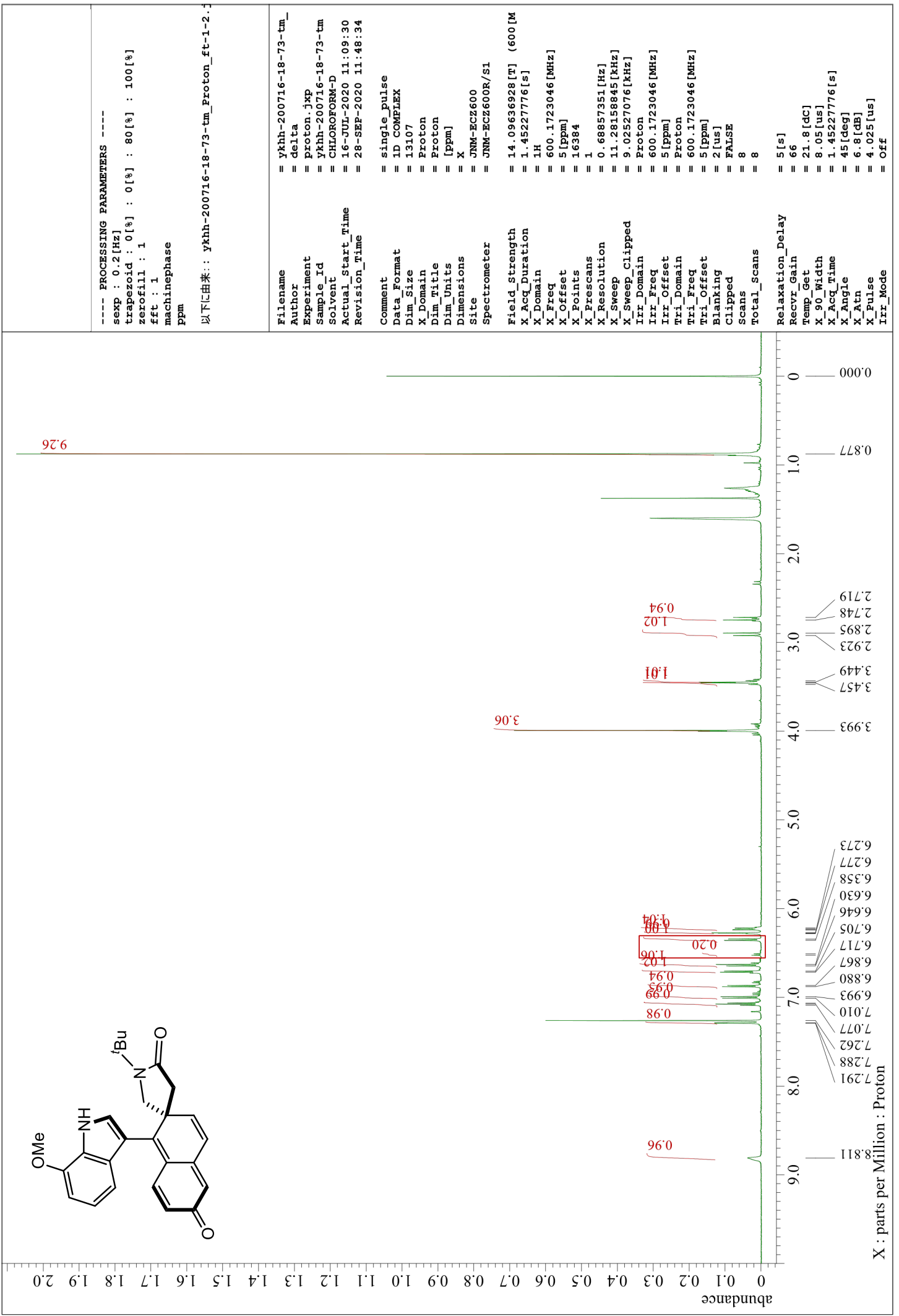




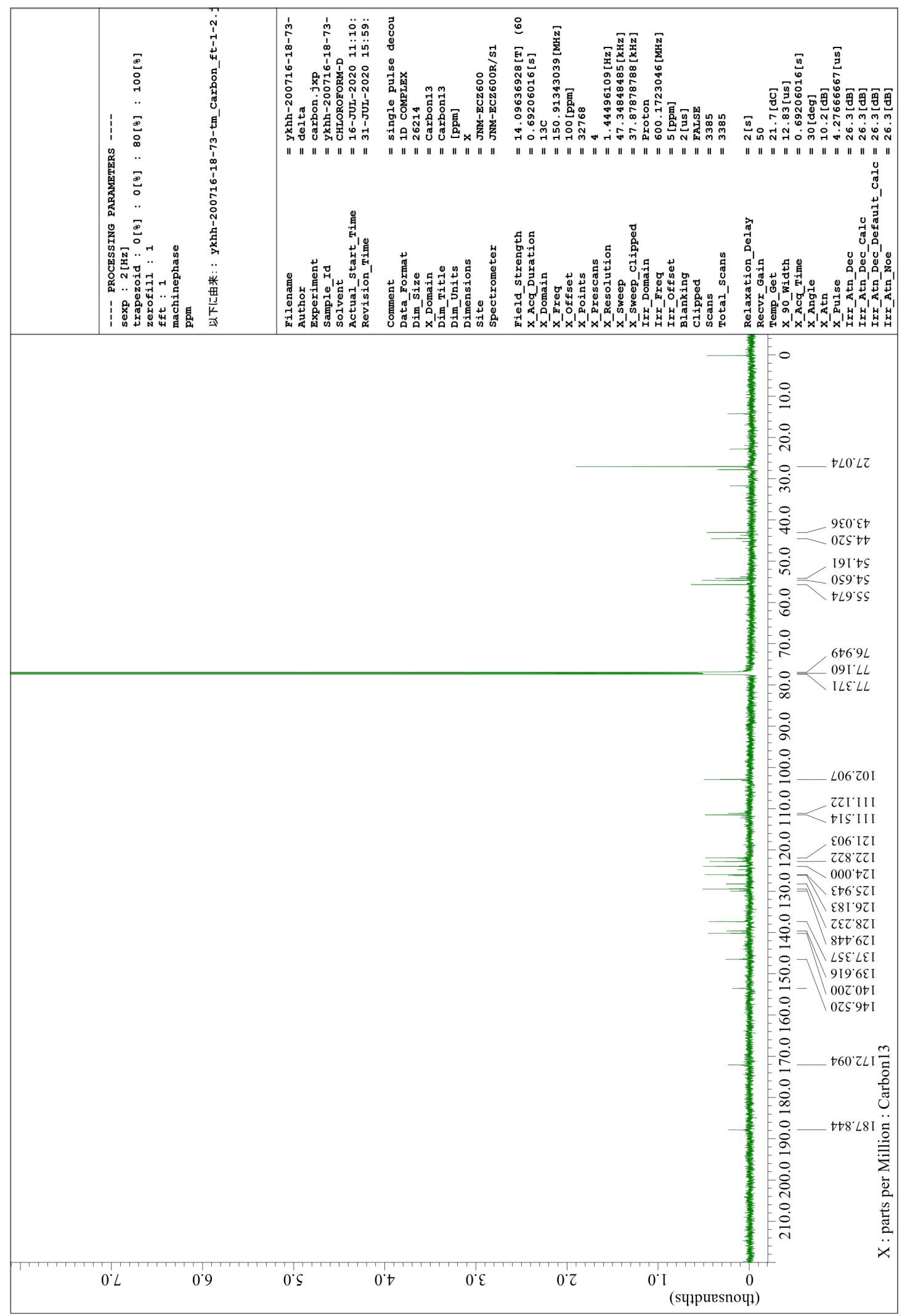


Supporting Information

$9 c$

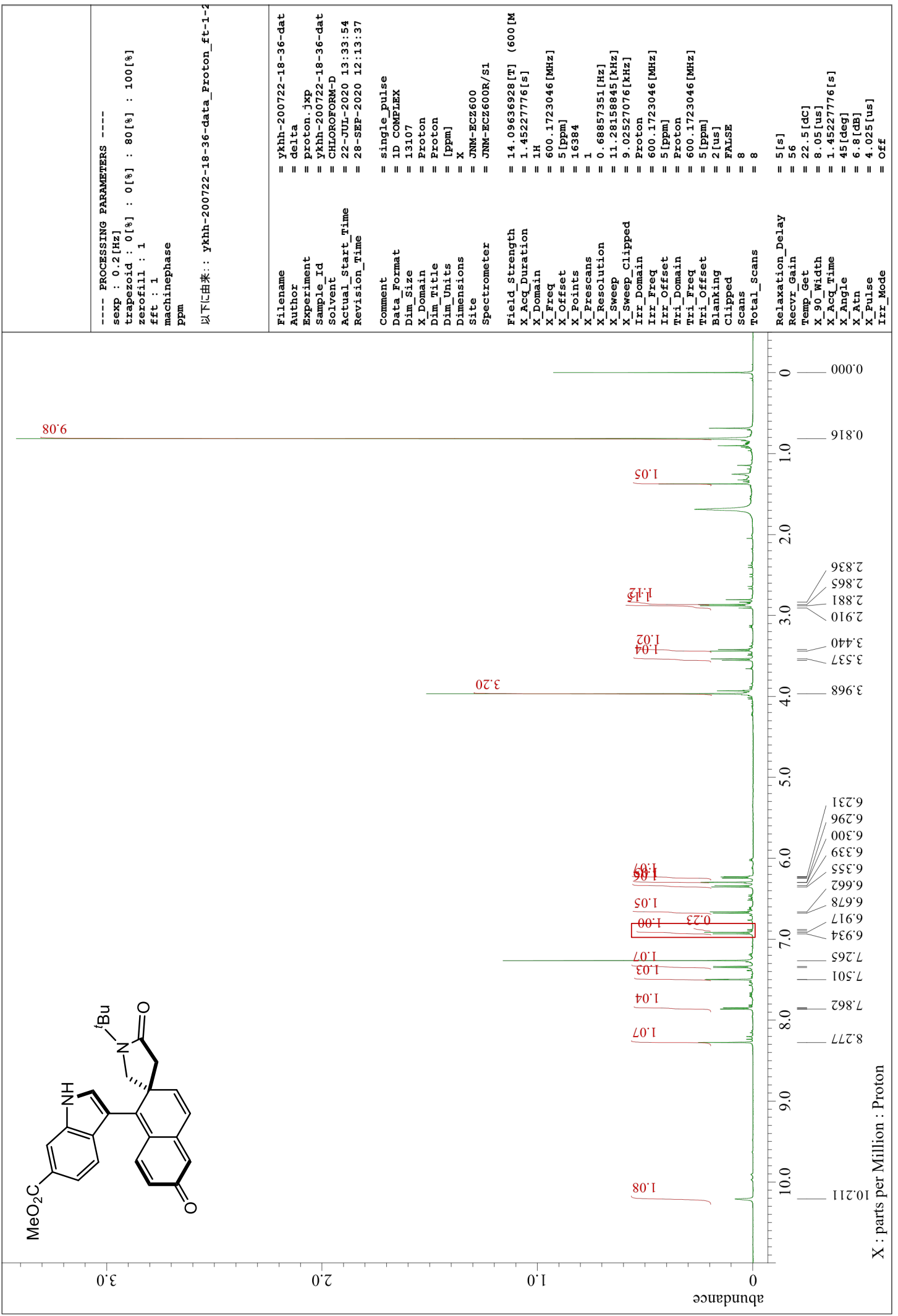




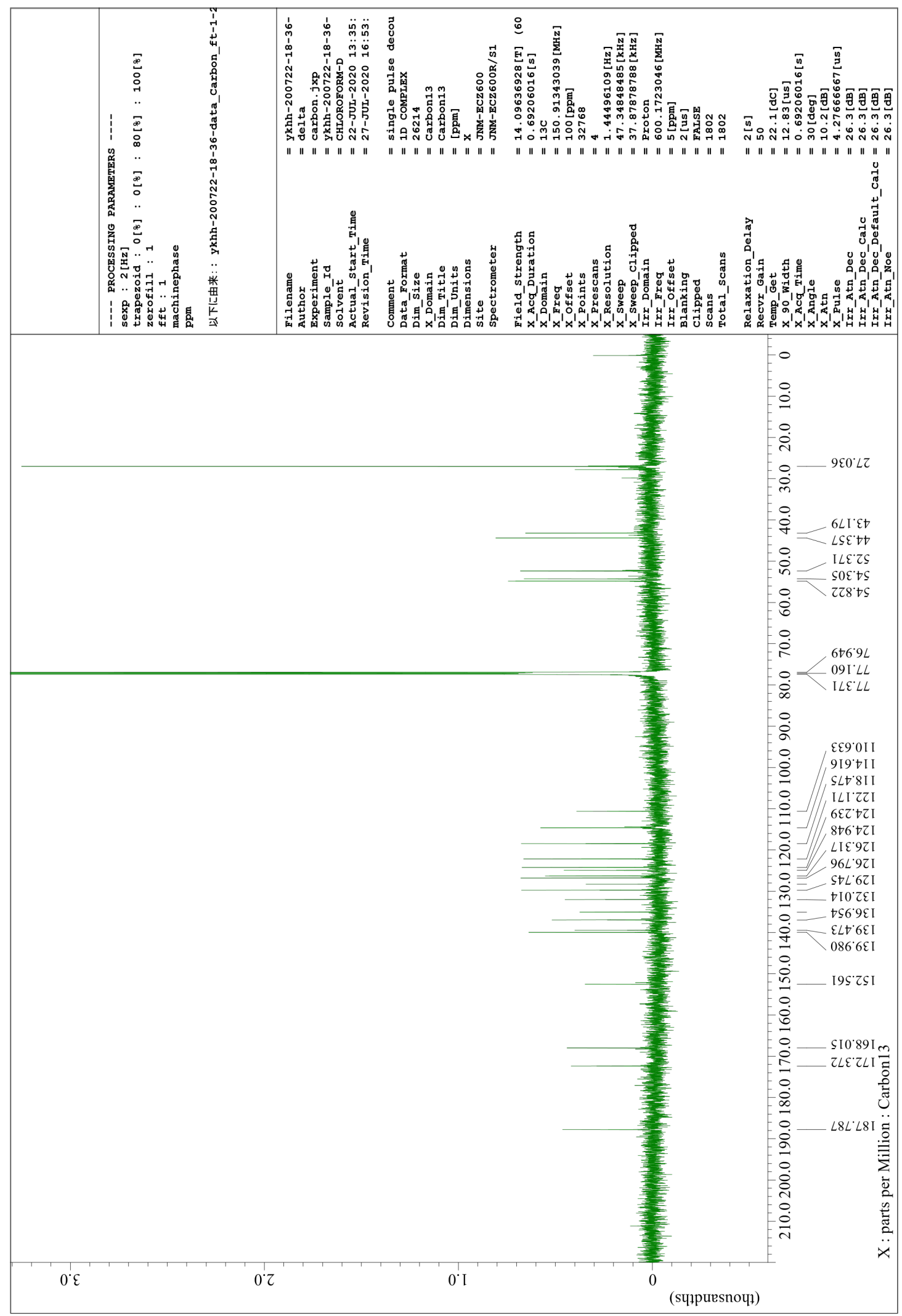


$9 d$

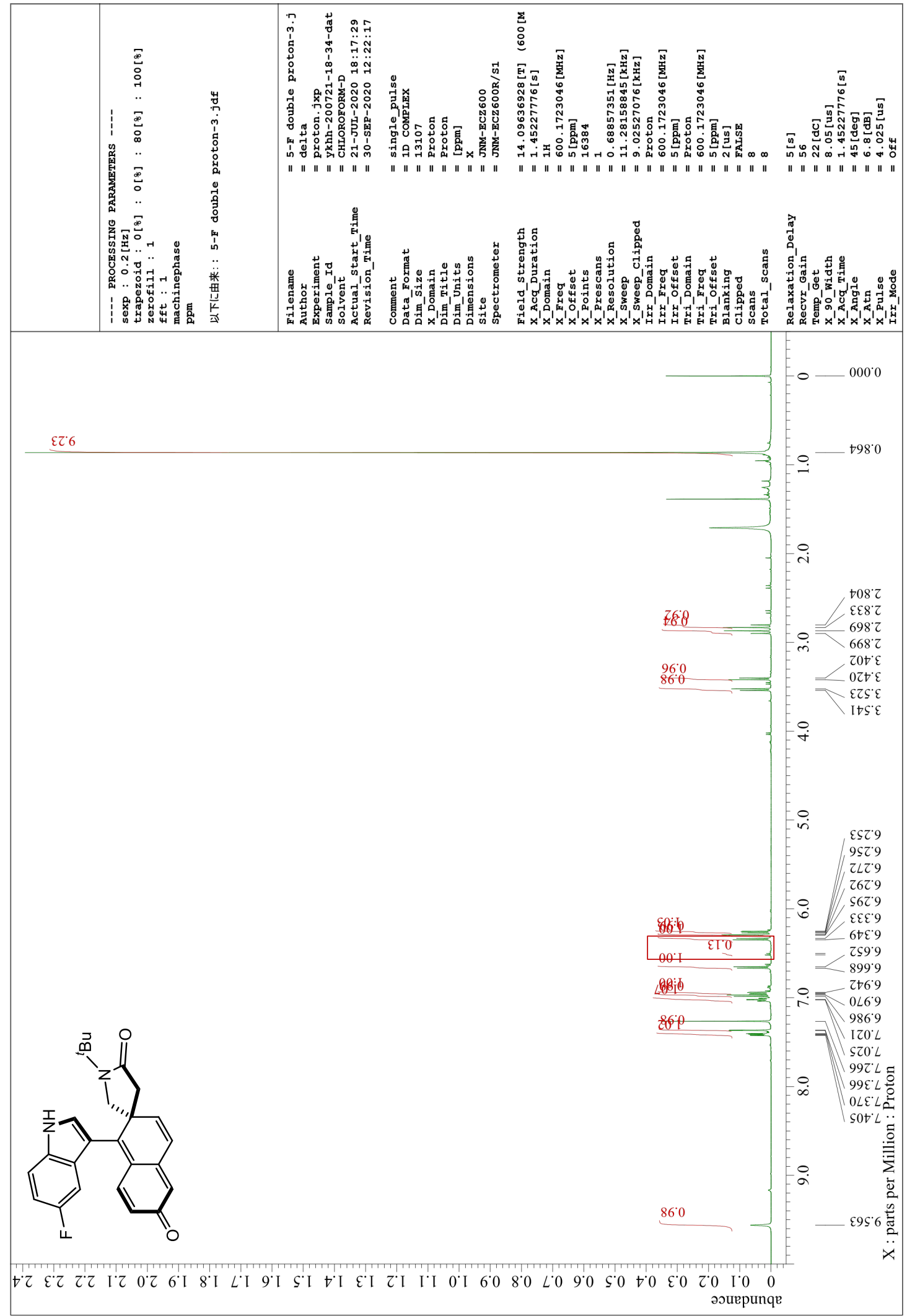




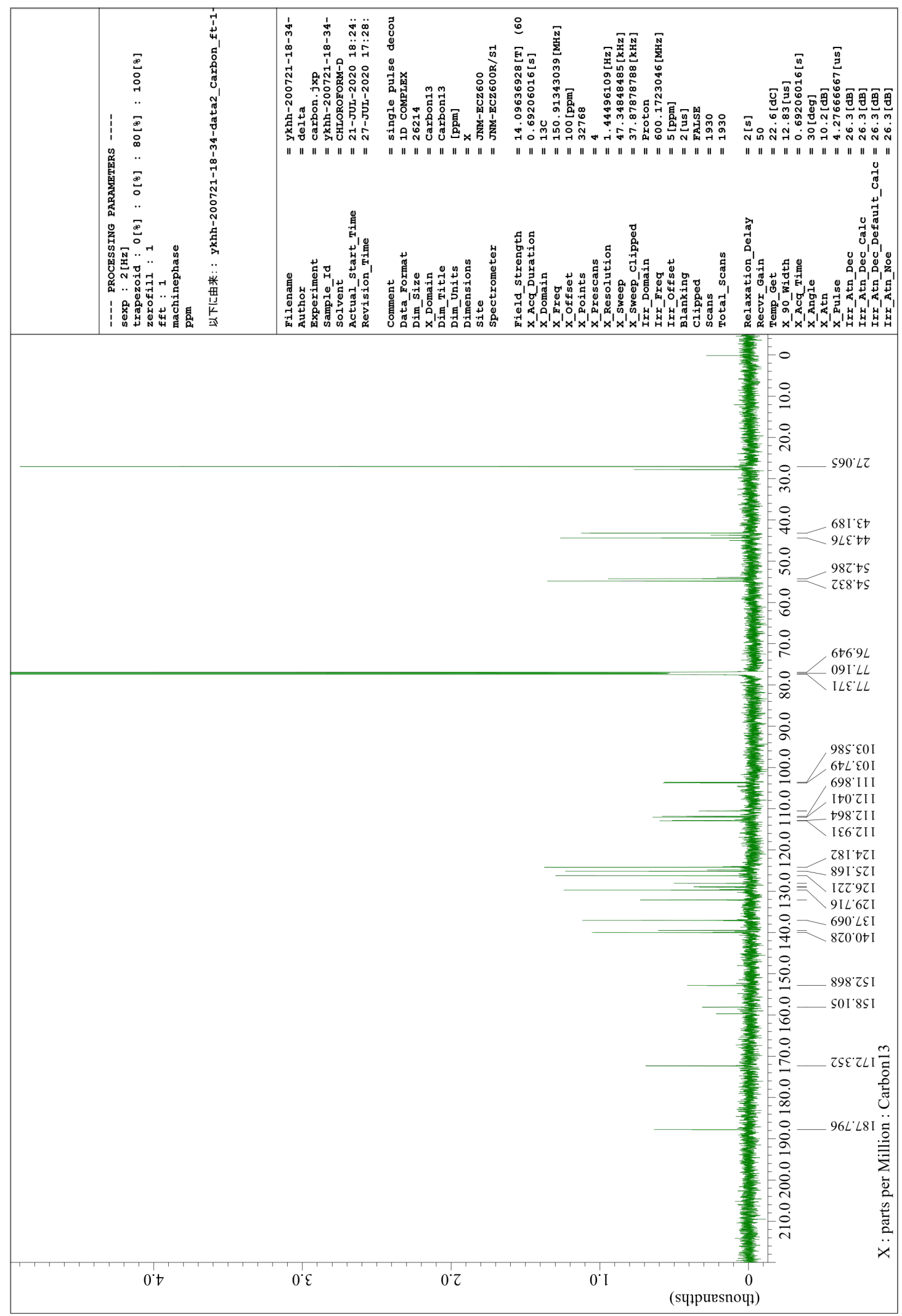




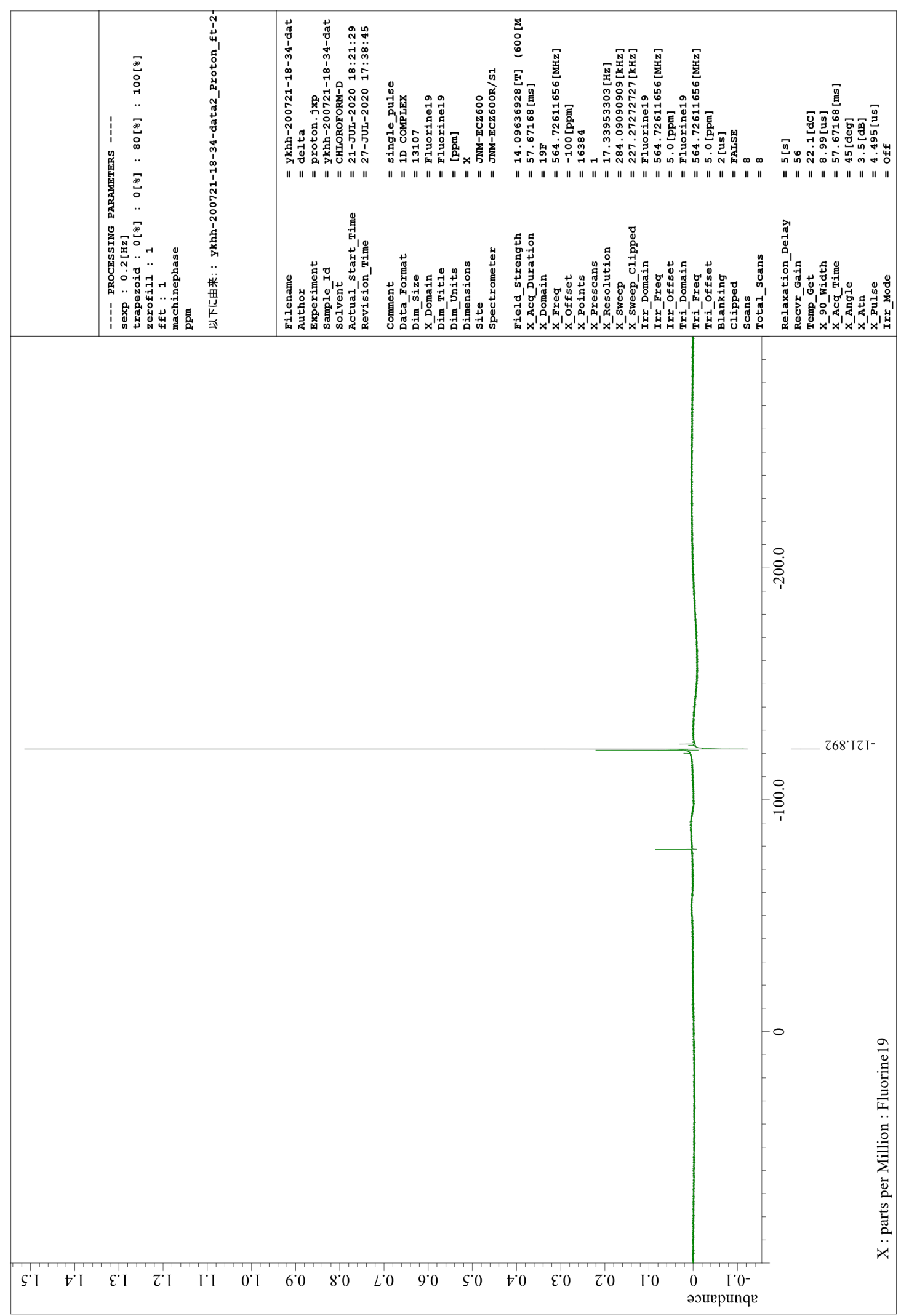


$9 e$

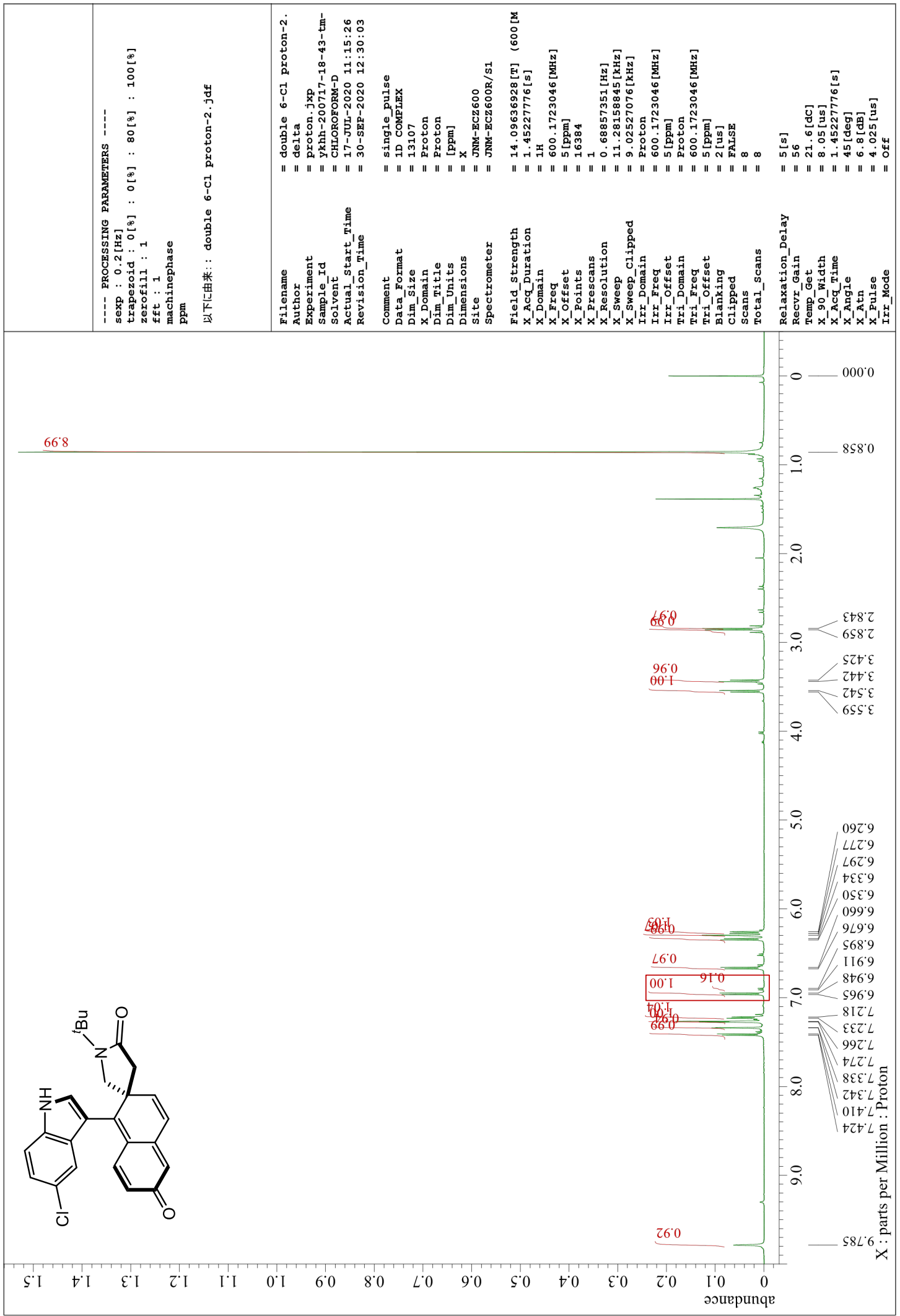




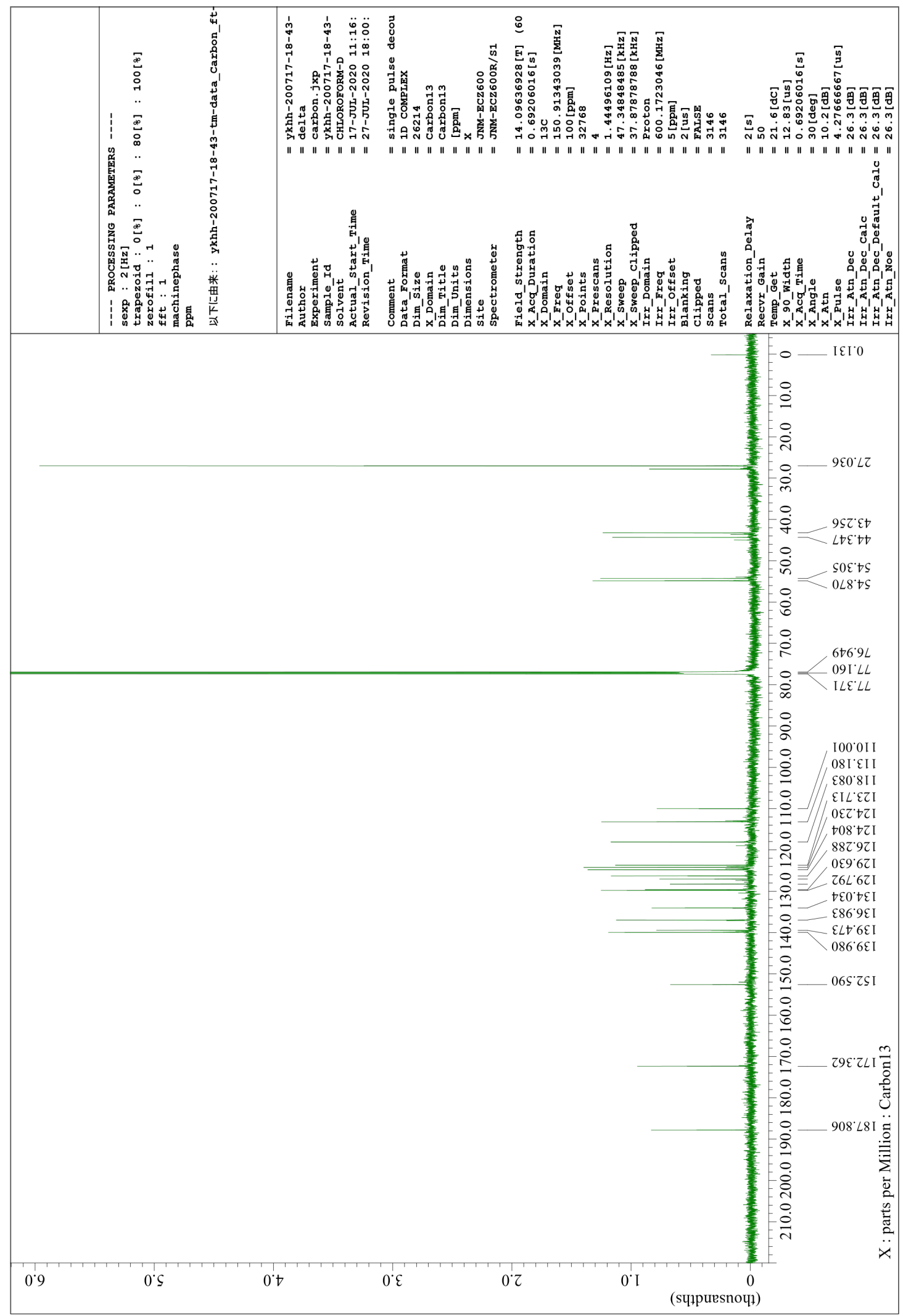




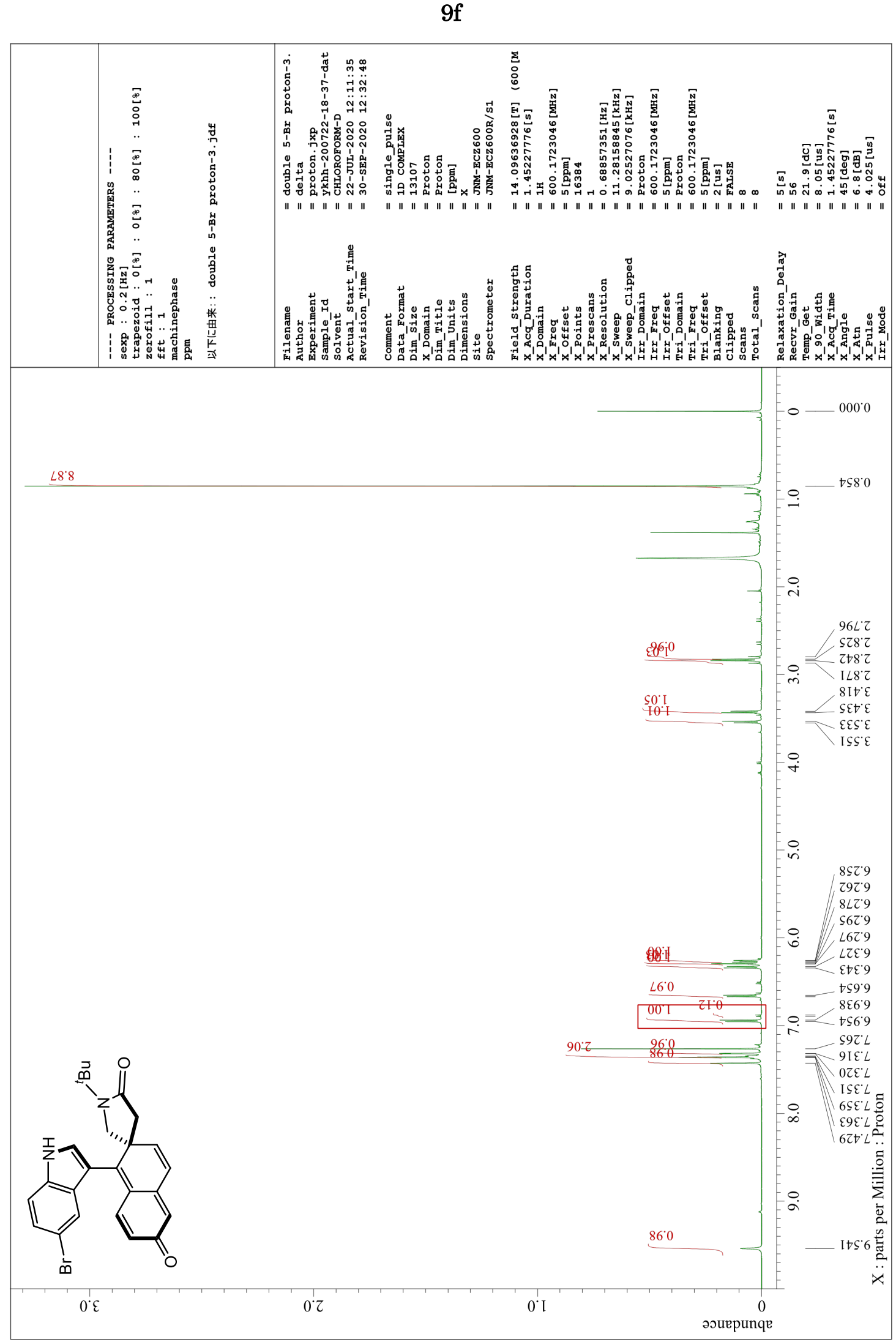




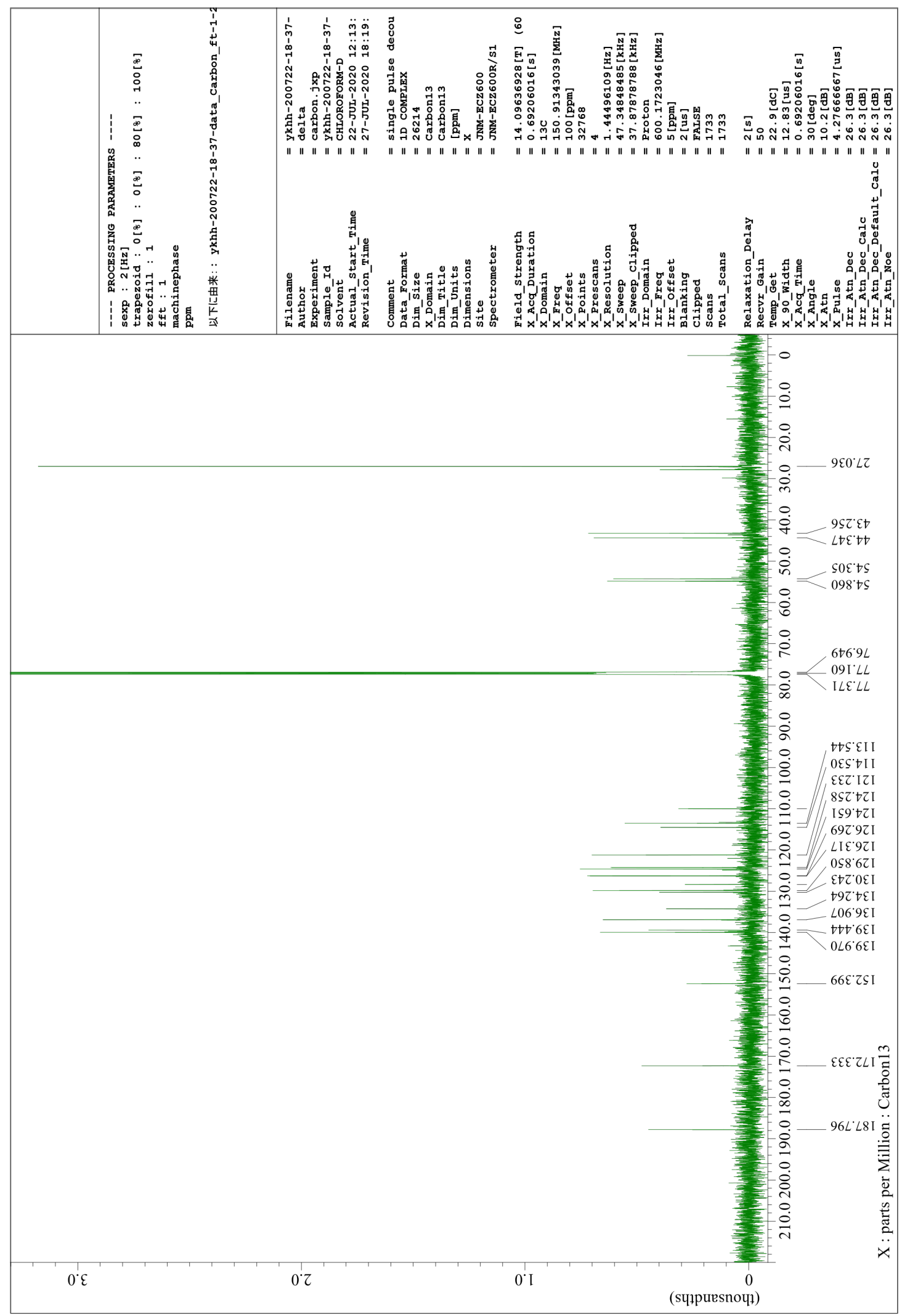


Supporting Information

\section{$9 g$}

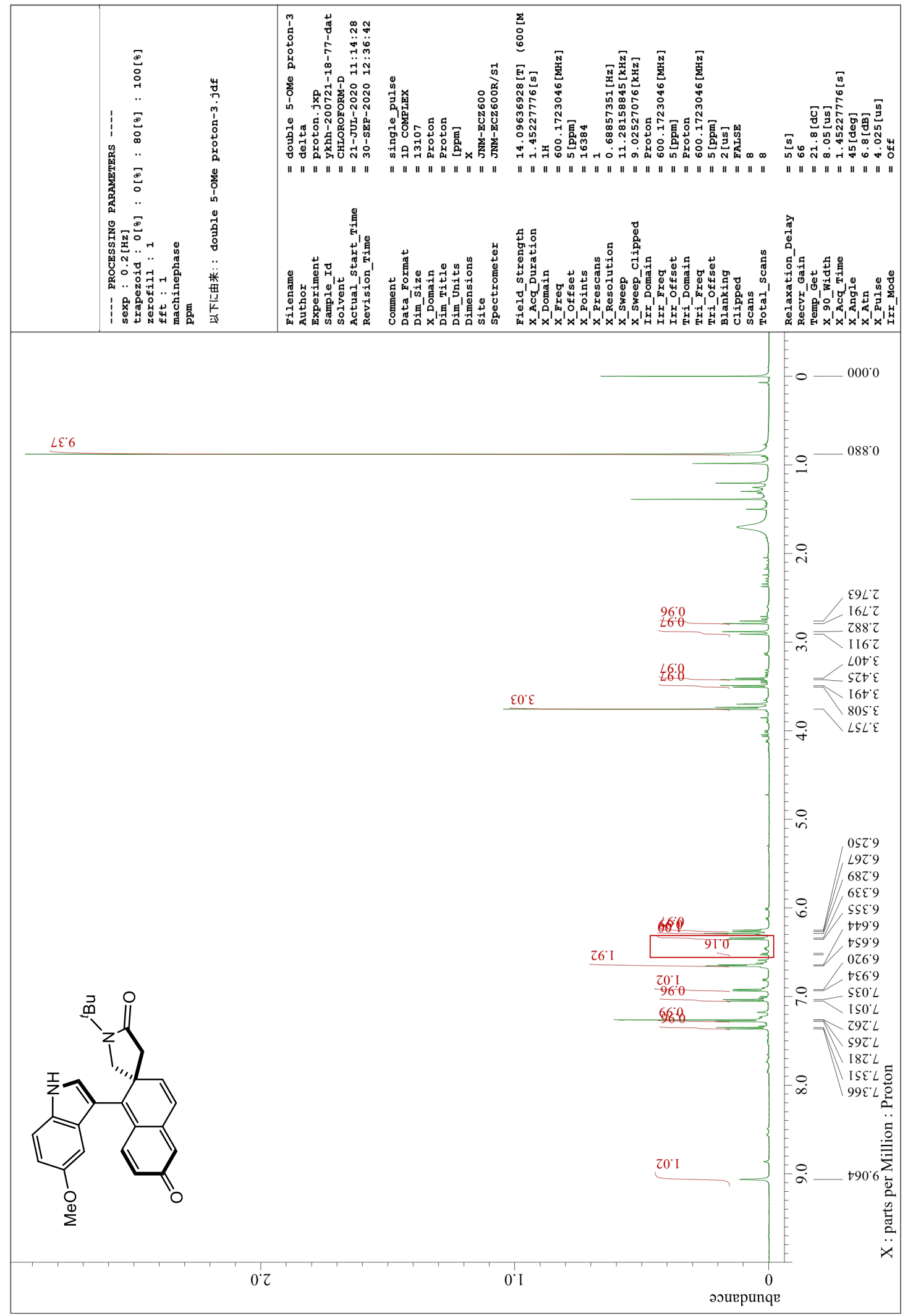




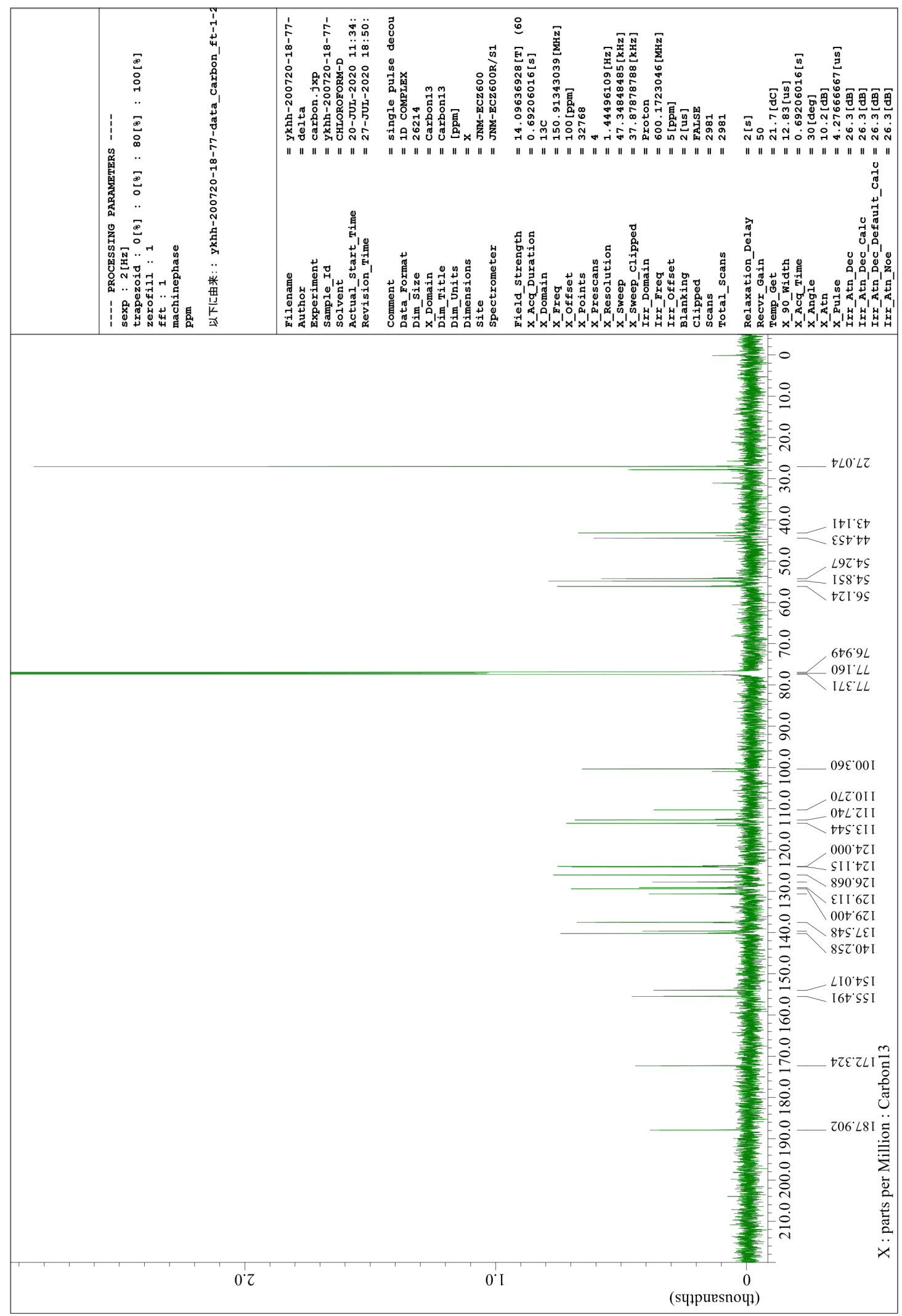


$9 \mathrm{~h}$

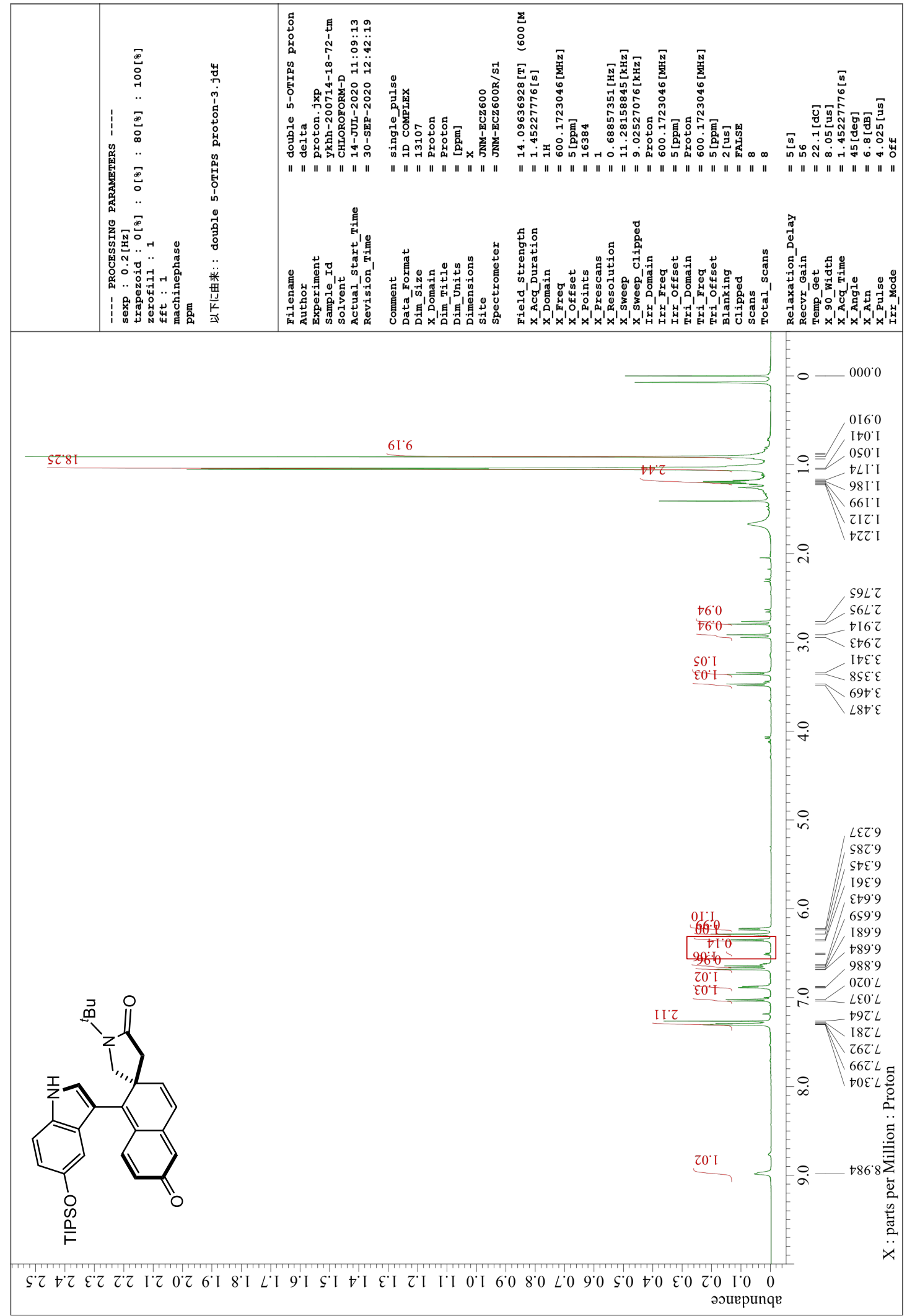




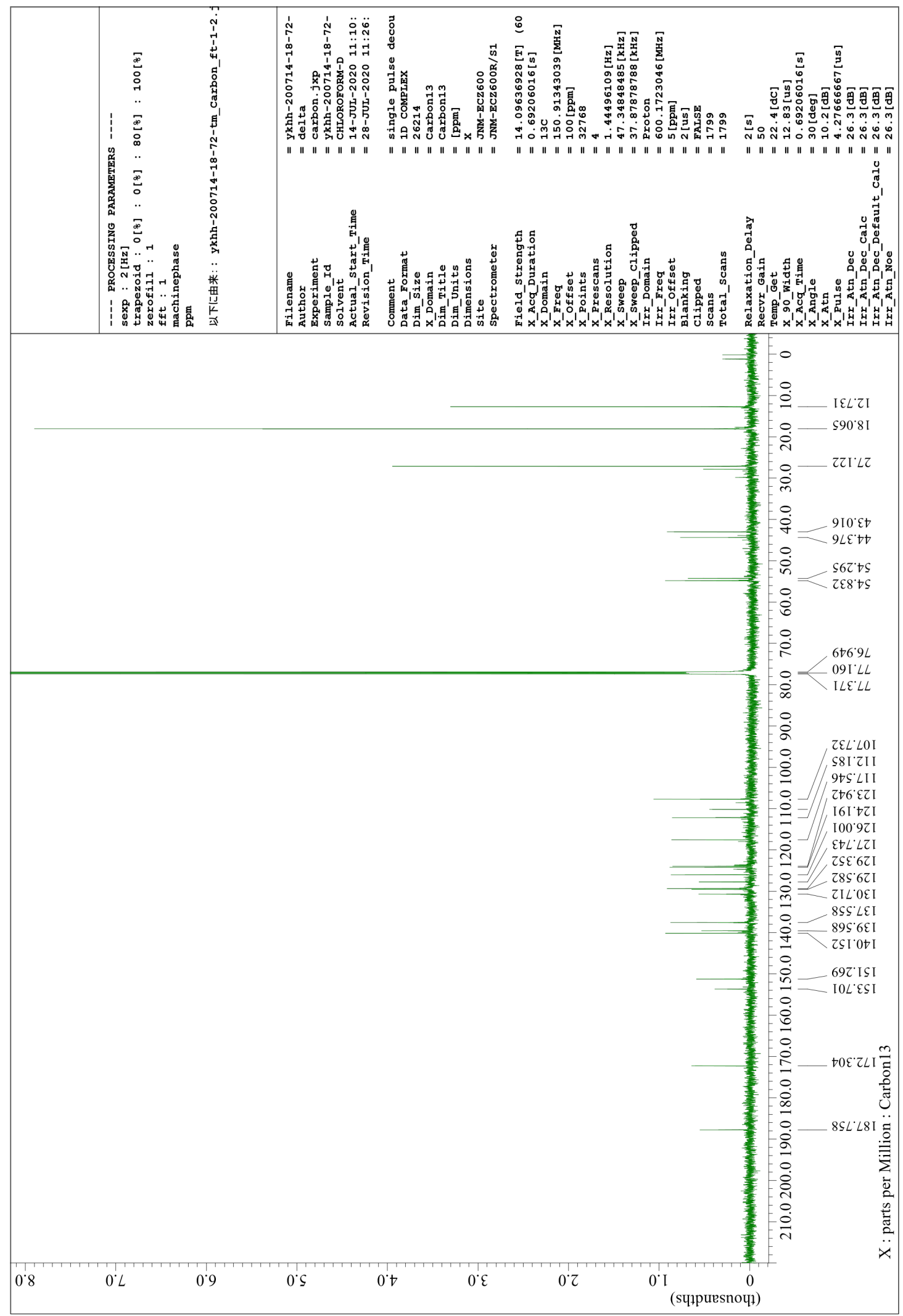


Supporting Information

9i (major diastereomer)

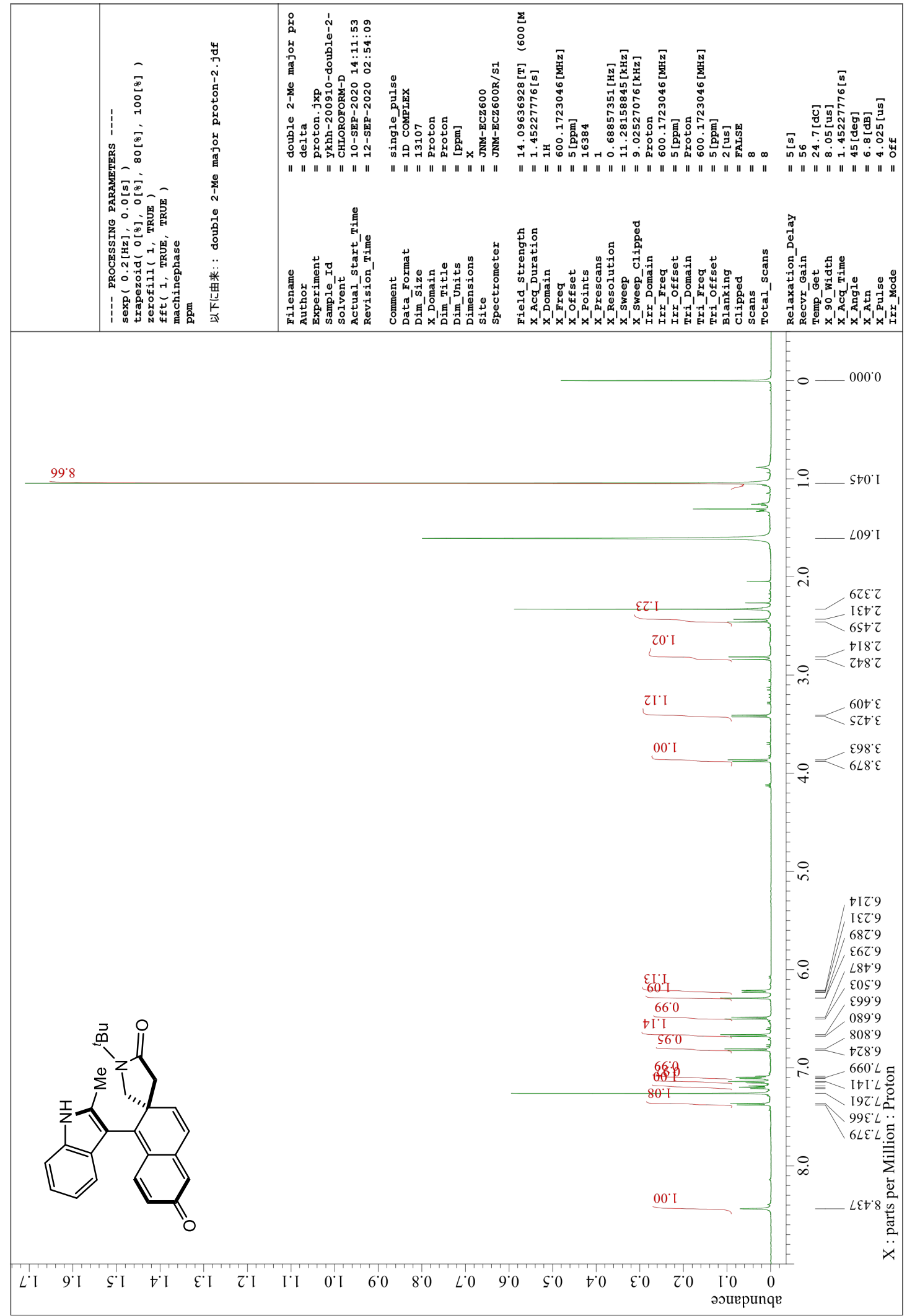




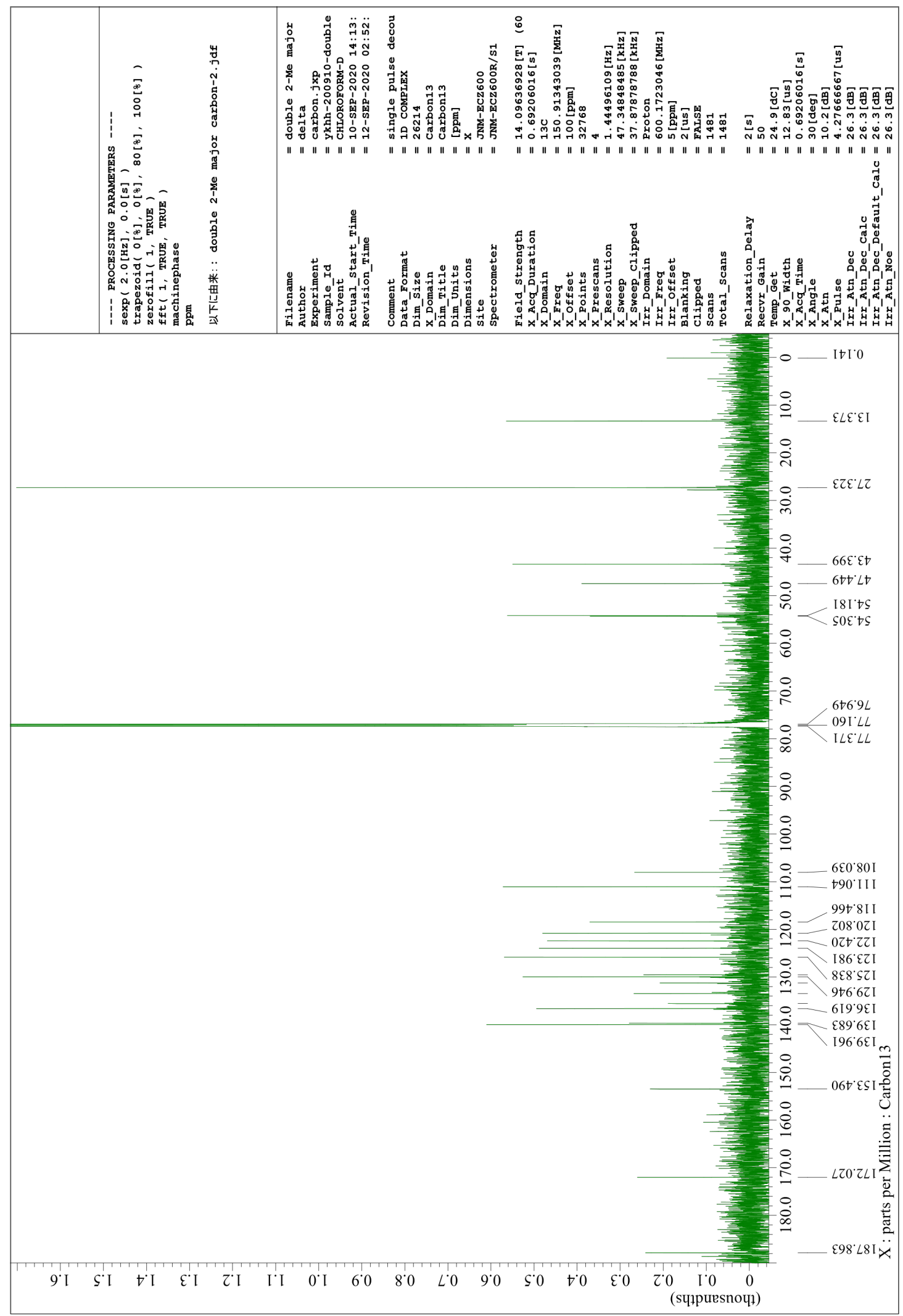


Supporting Information

9i (minor diastereomer)

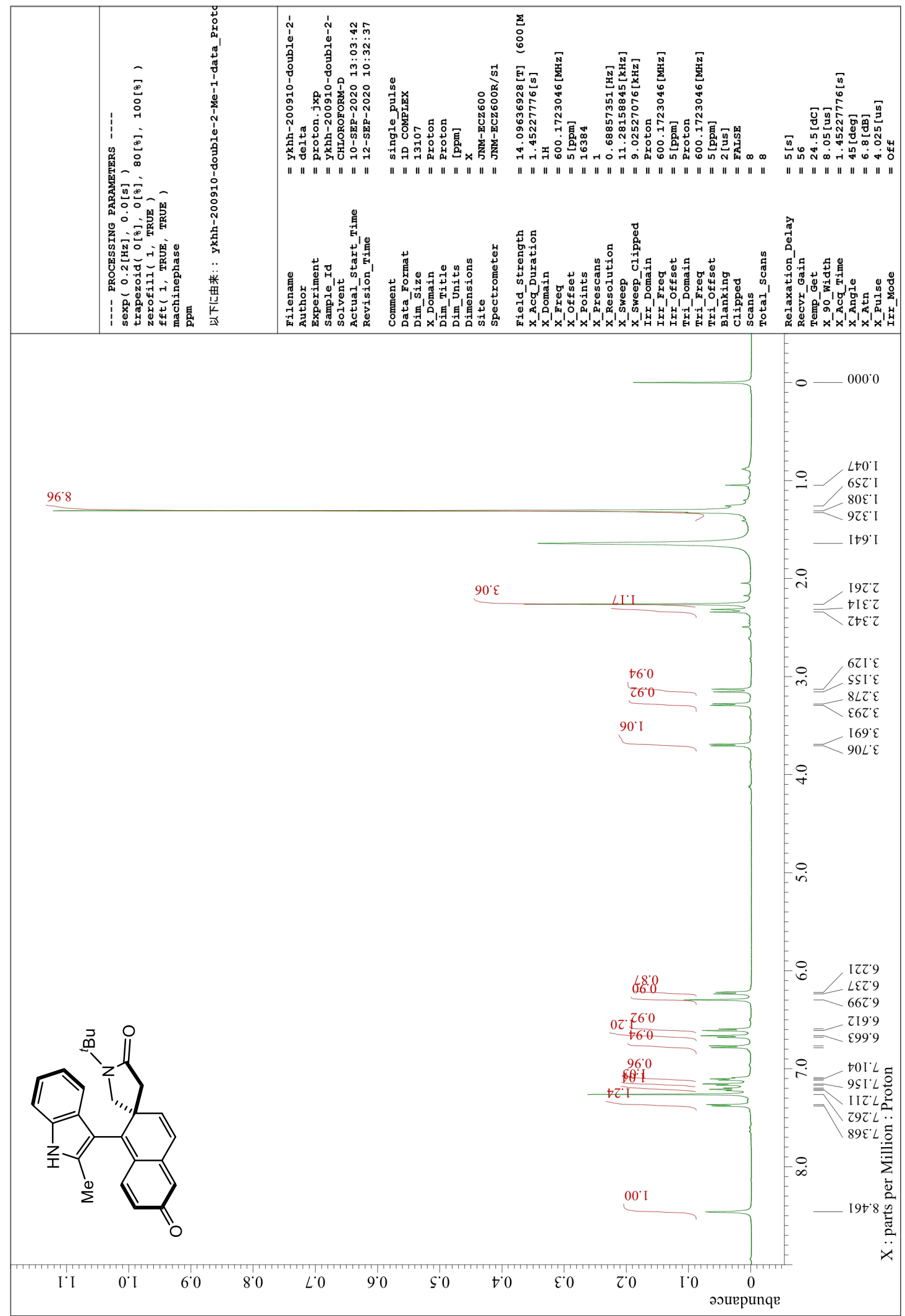




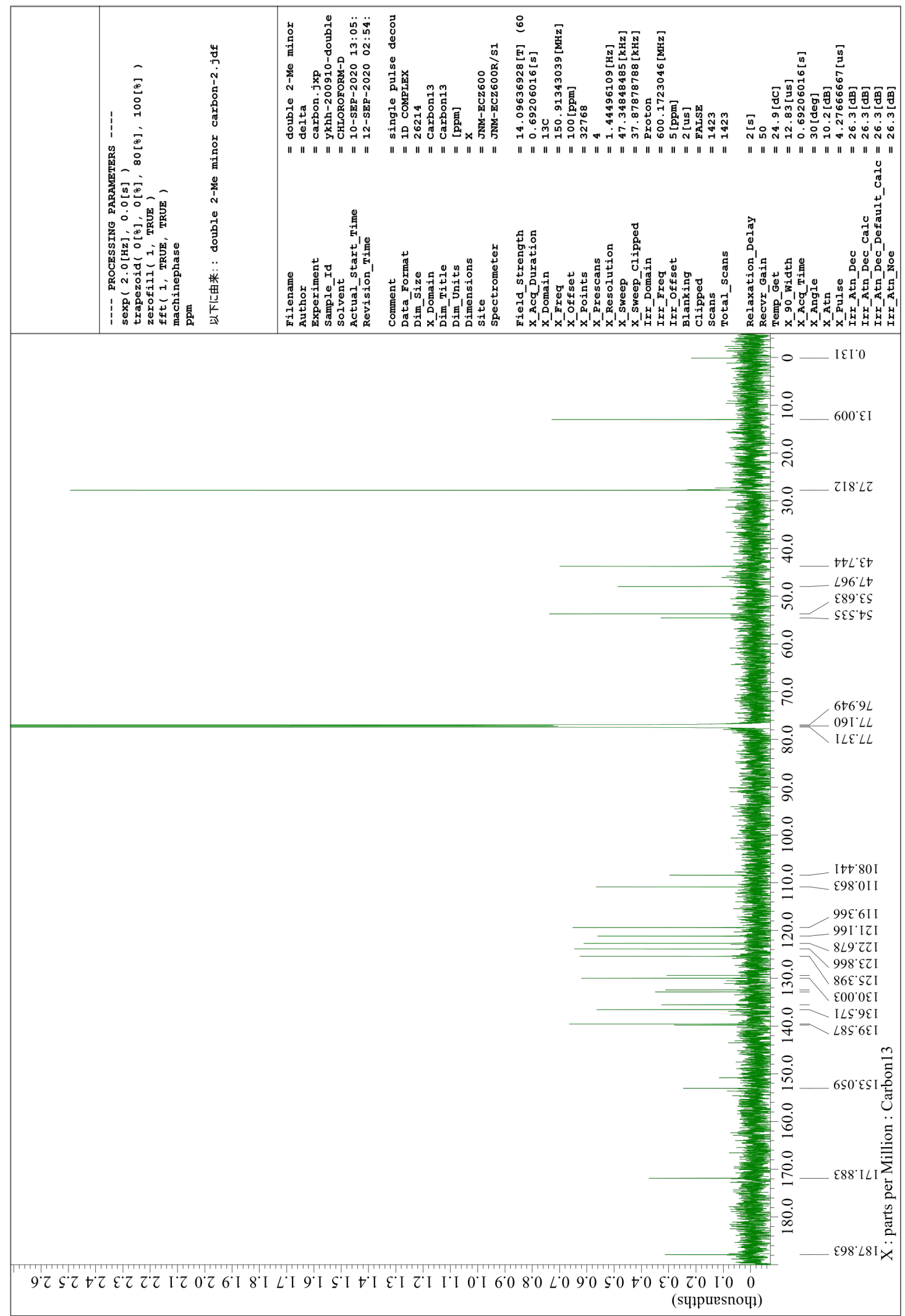




\section{9j}

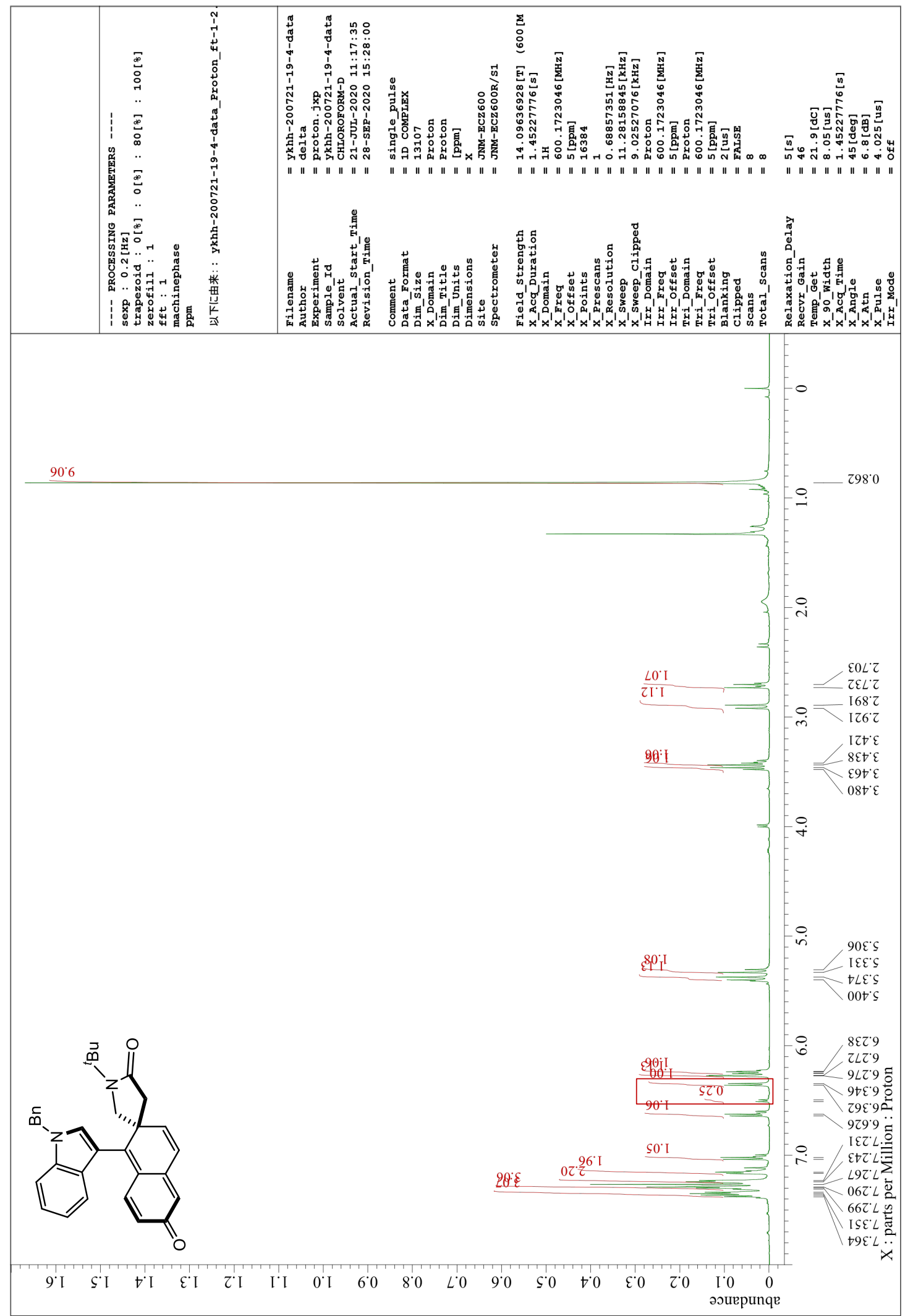




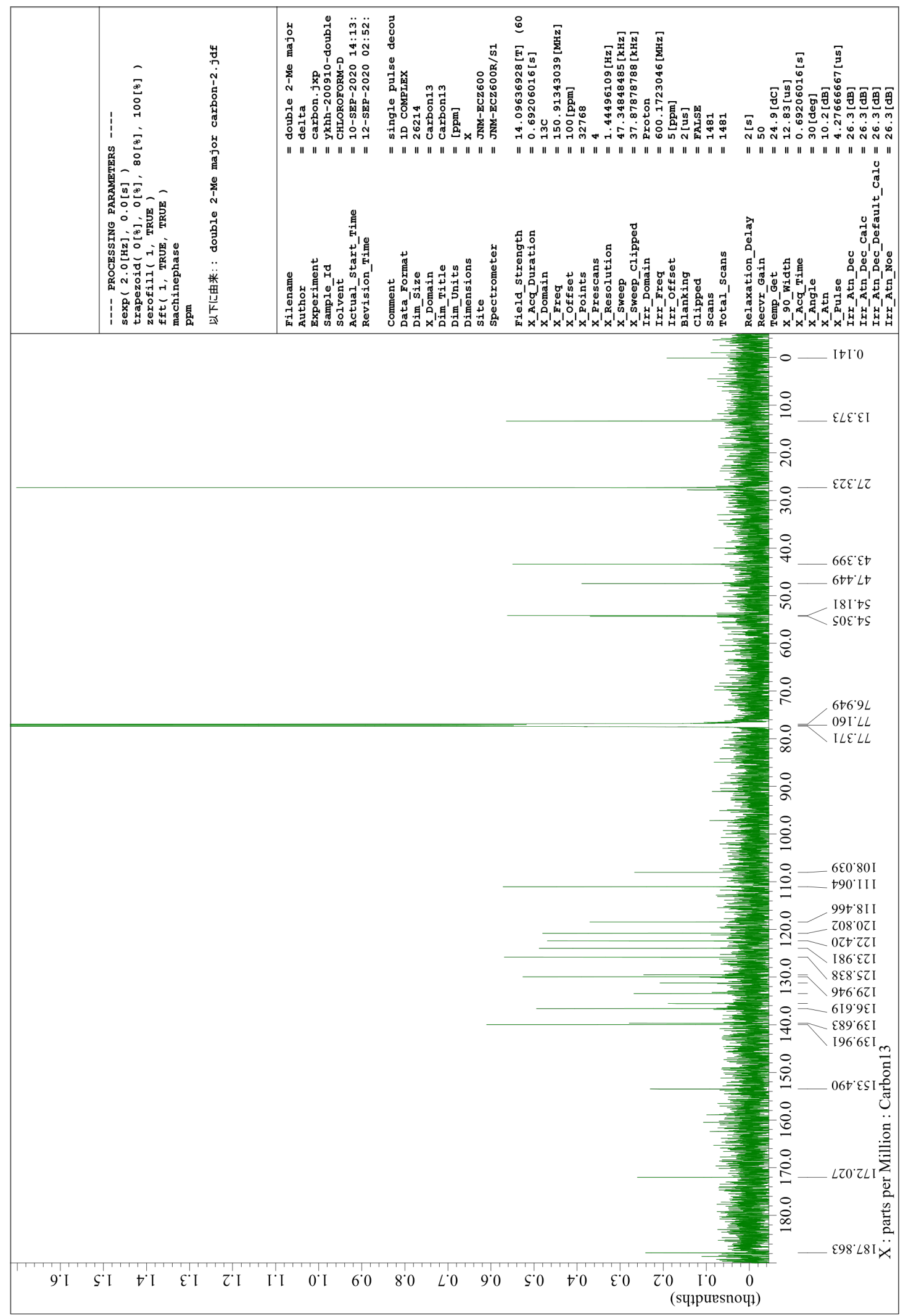


$9 k$

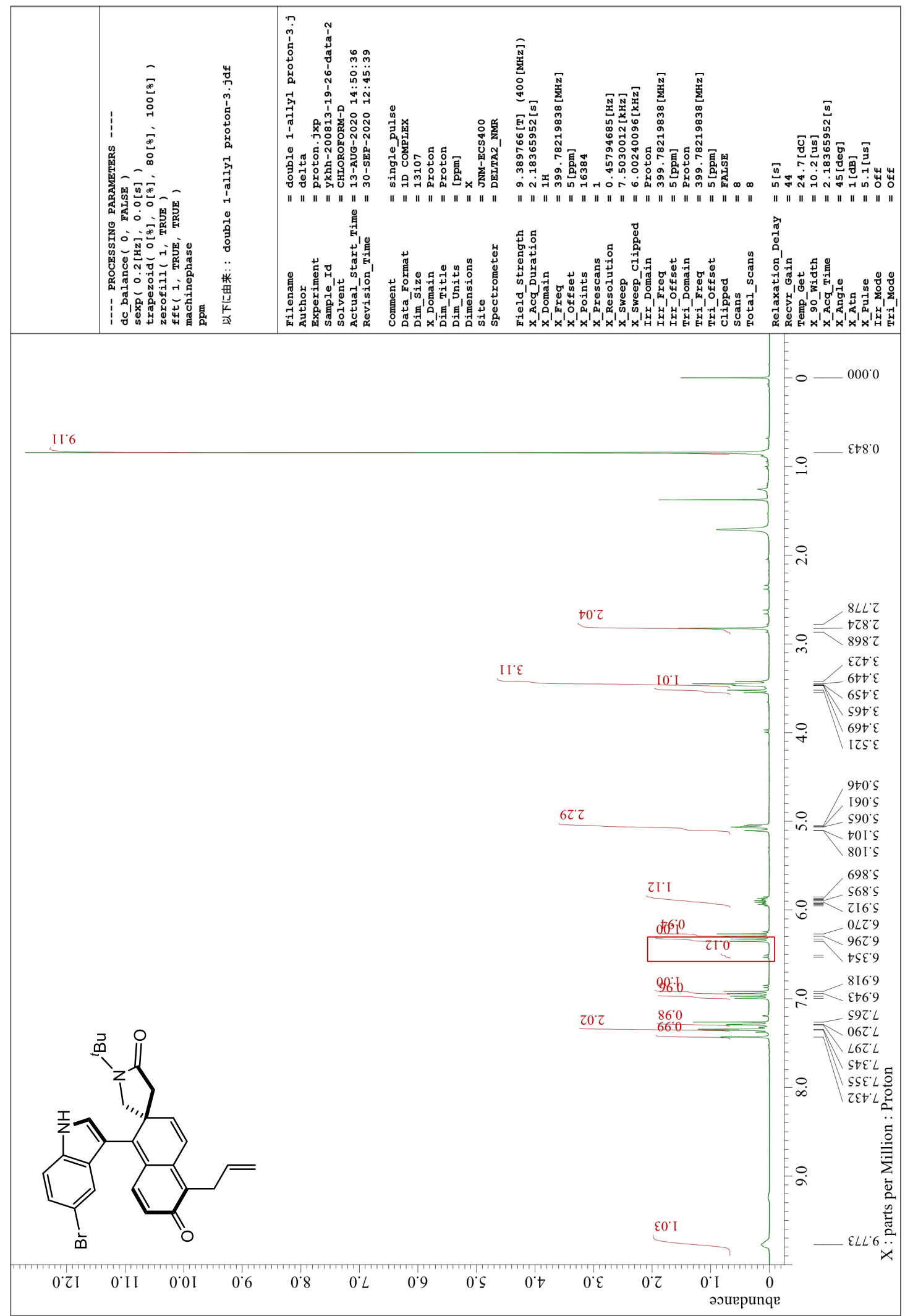




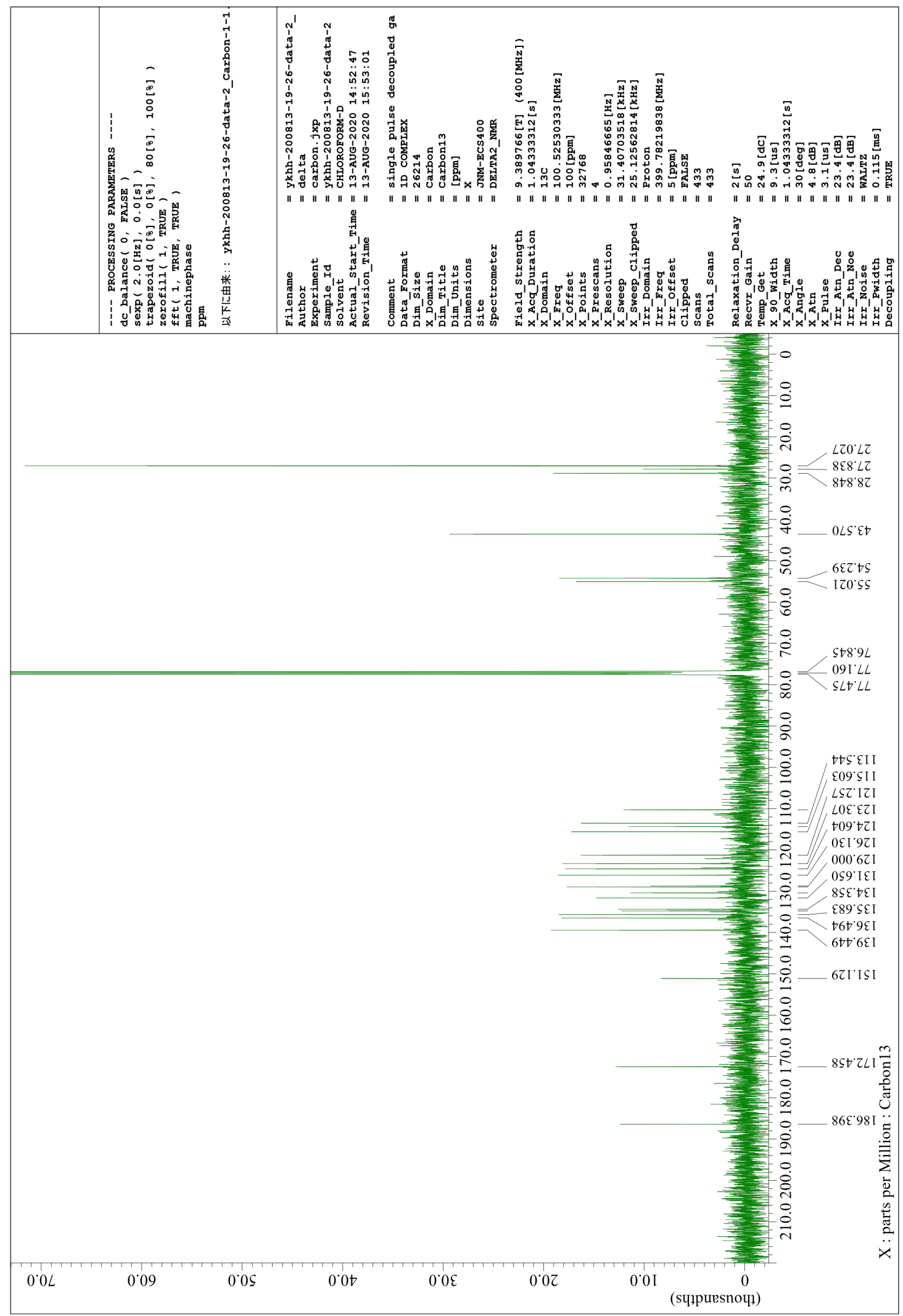


Supporting Information

91

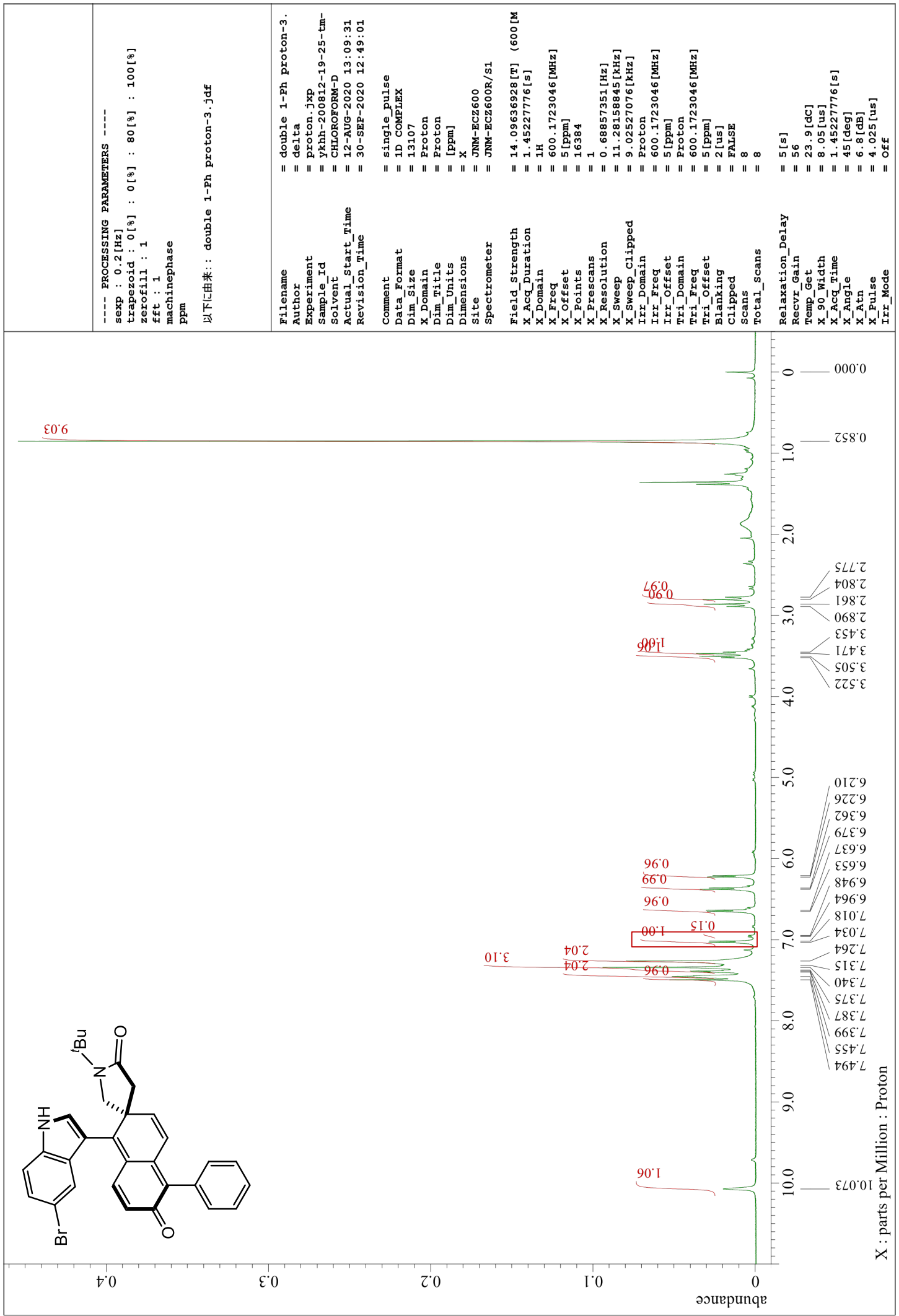




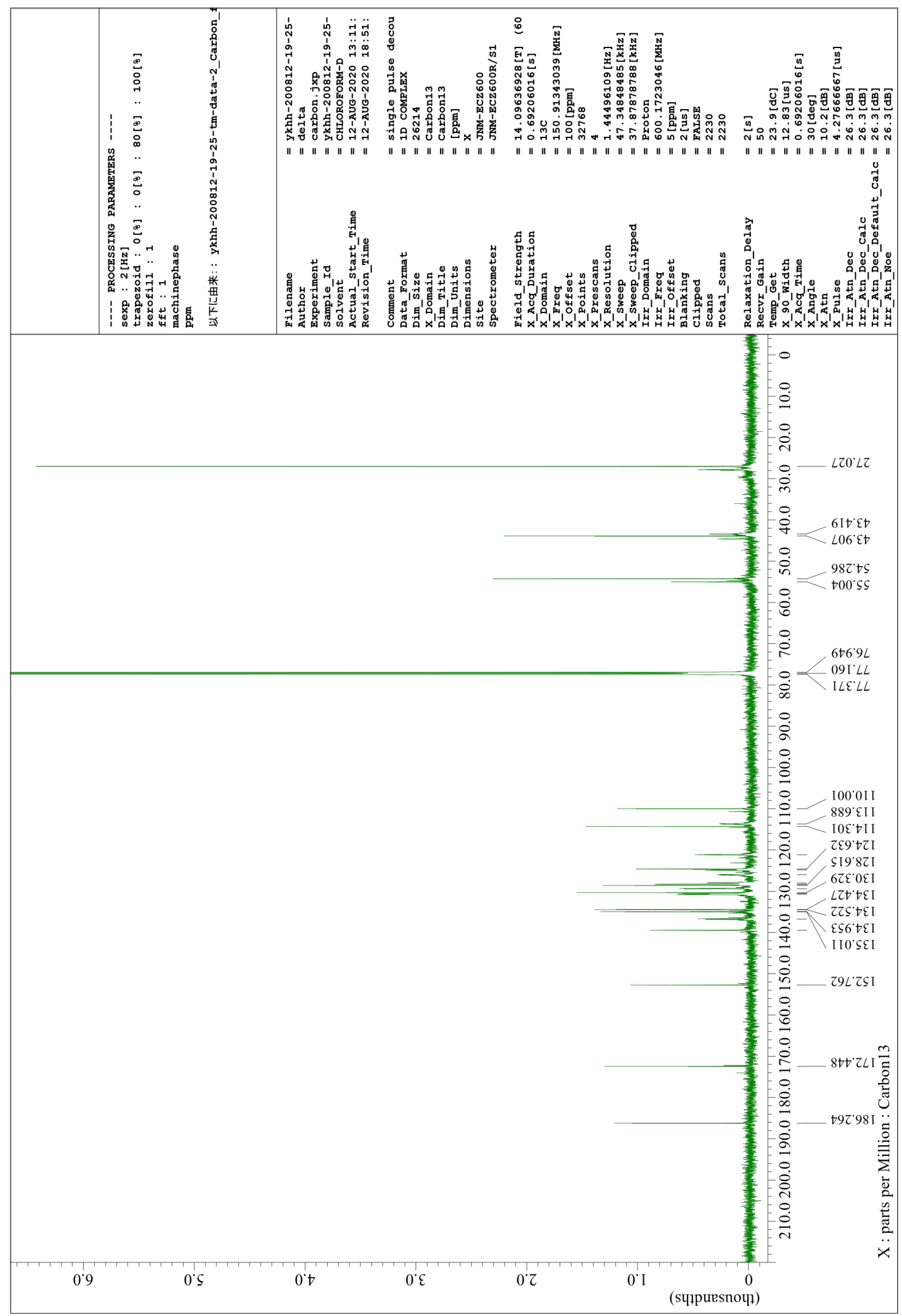


$10 \mathrm{a}$

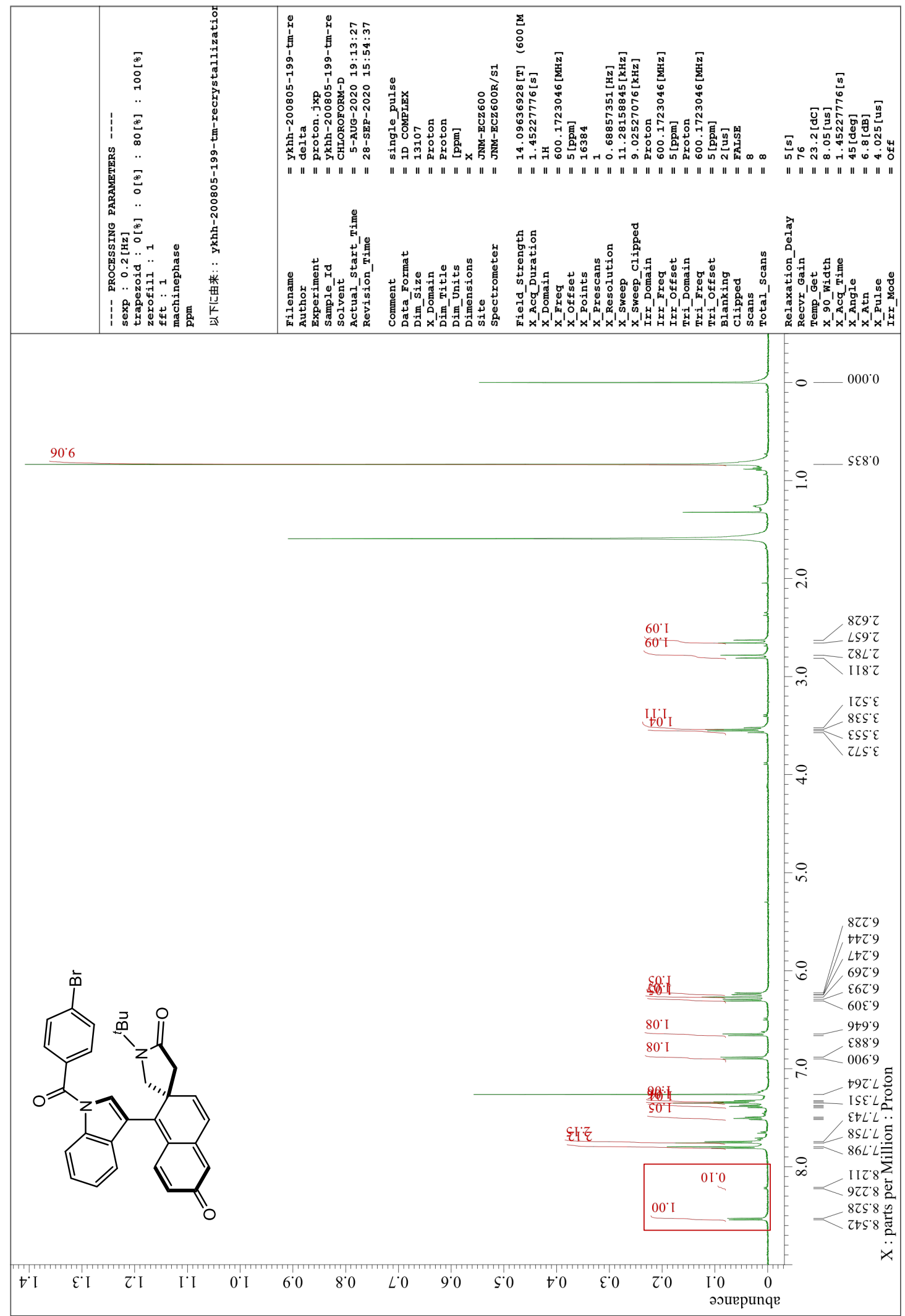




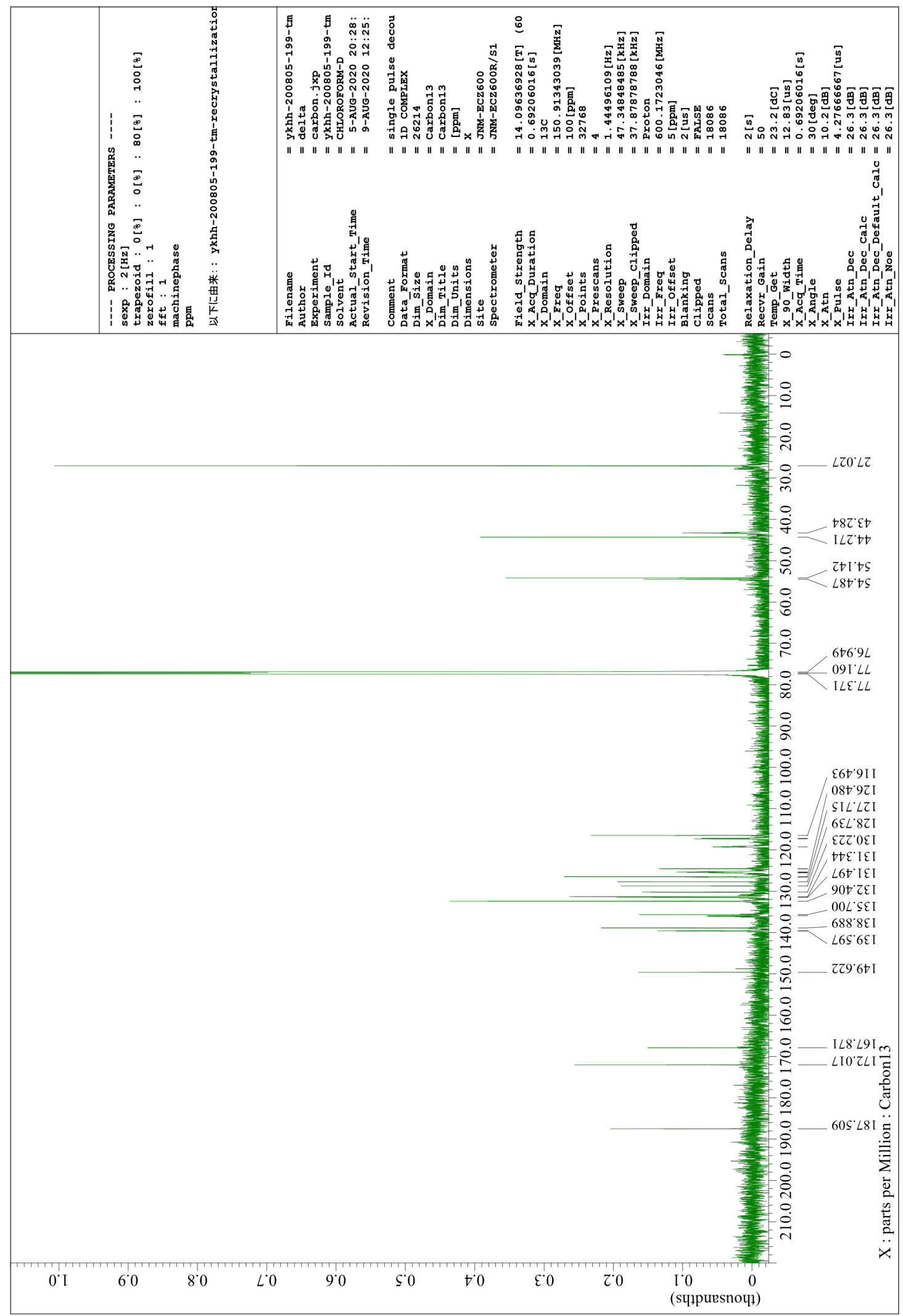


Supporting Information

$1 a$

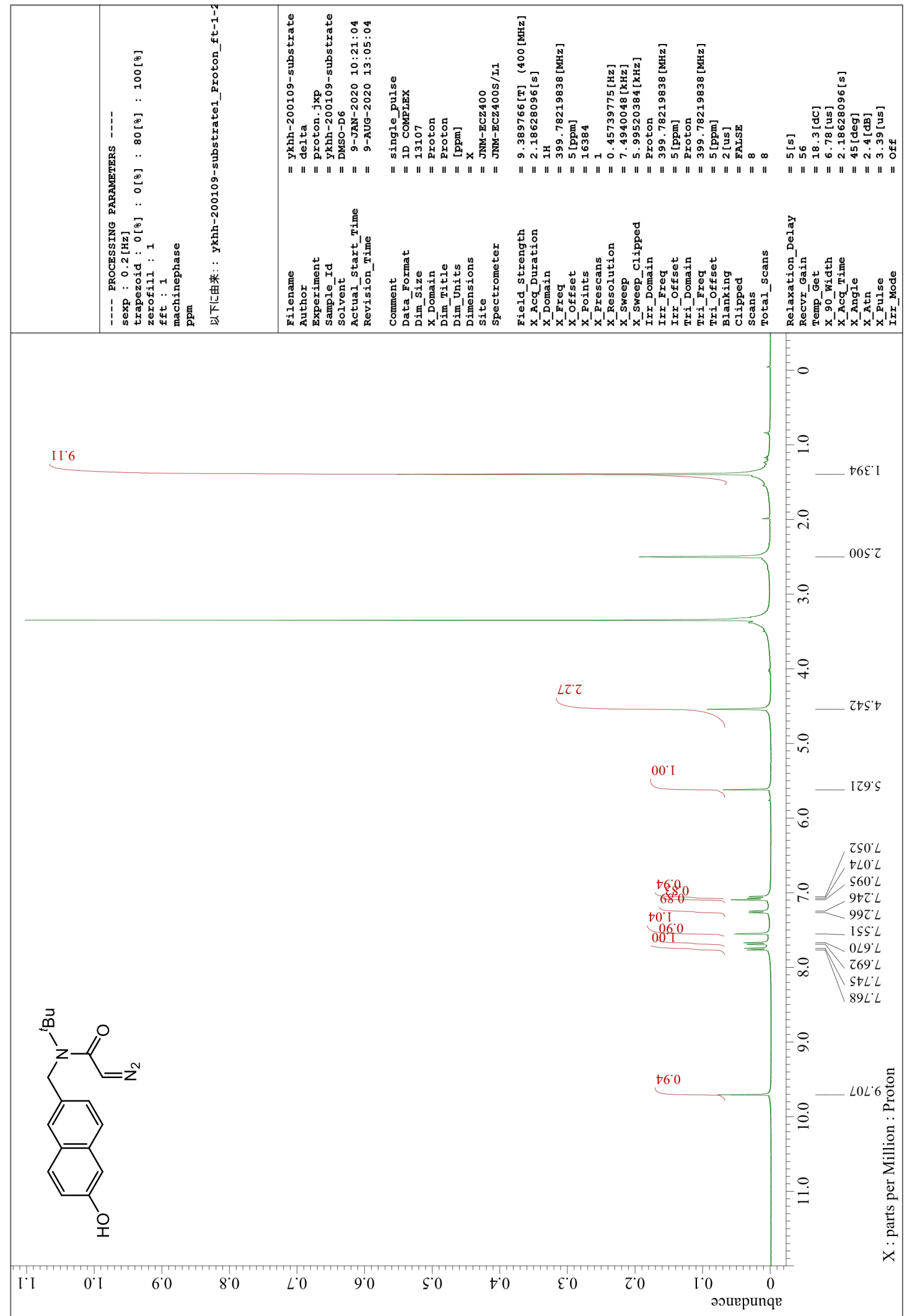




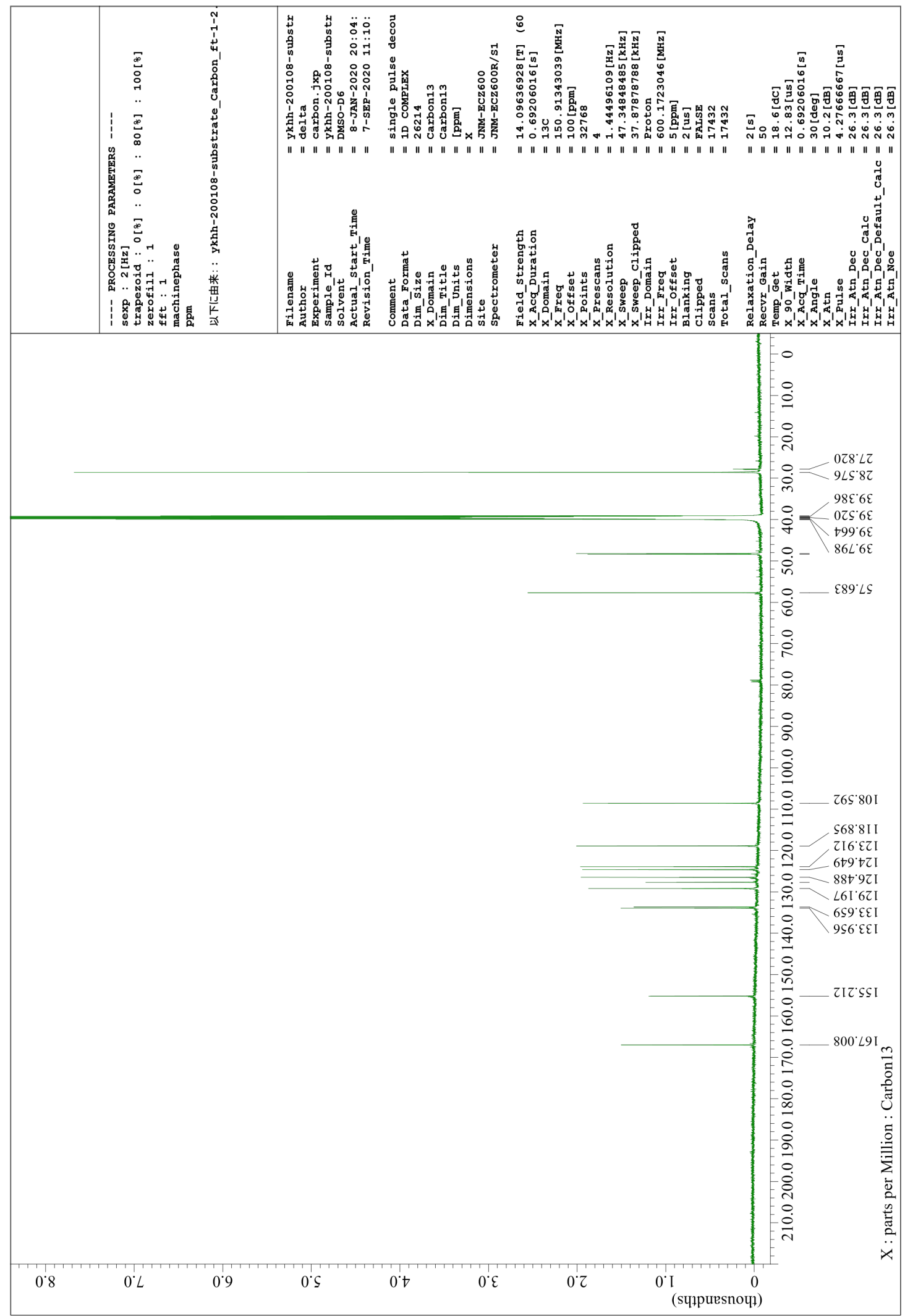


S1

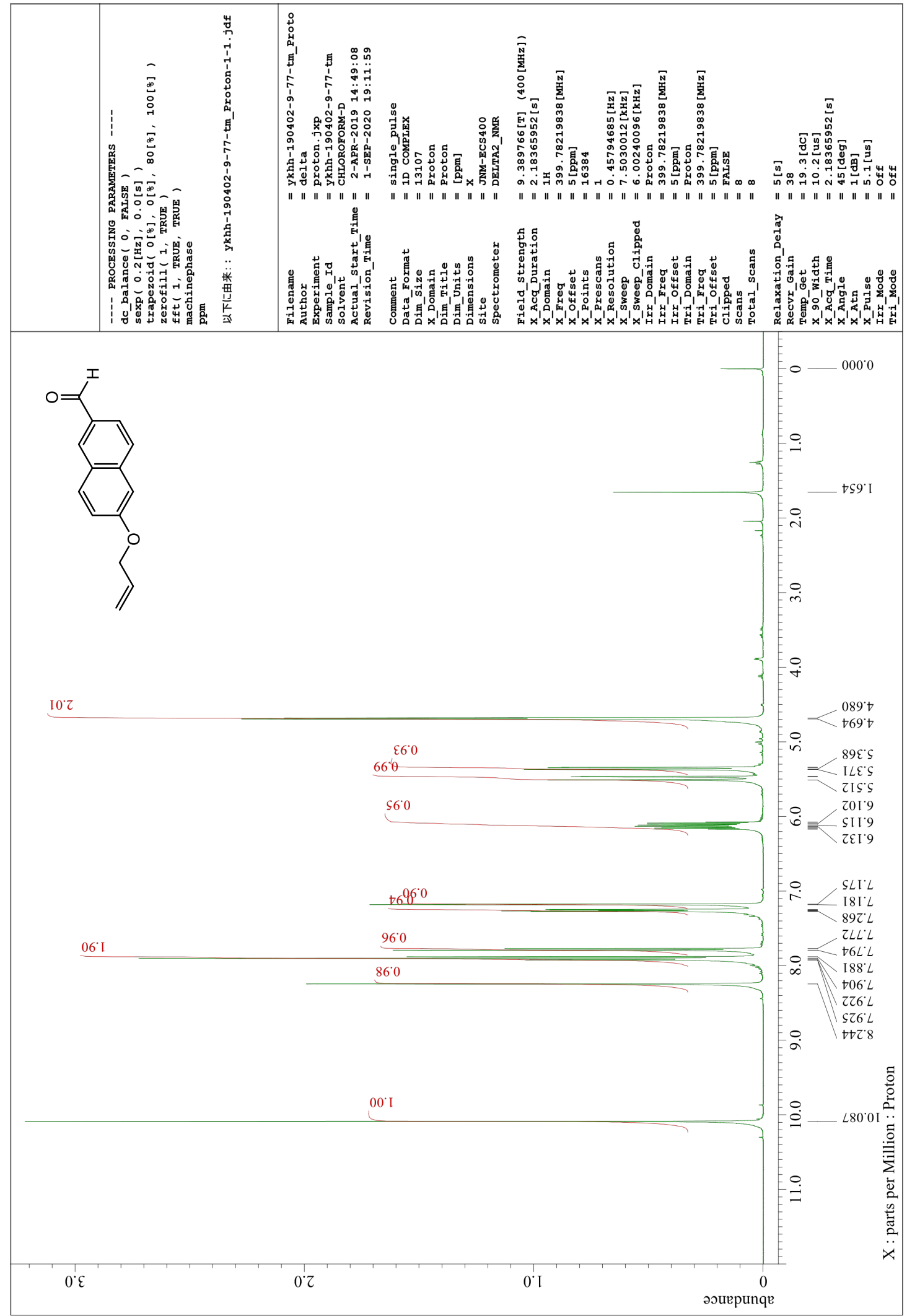


Supporting Information

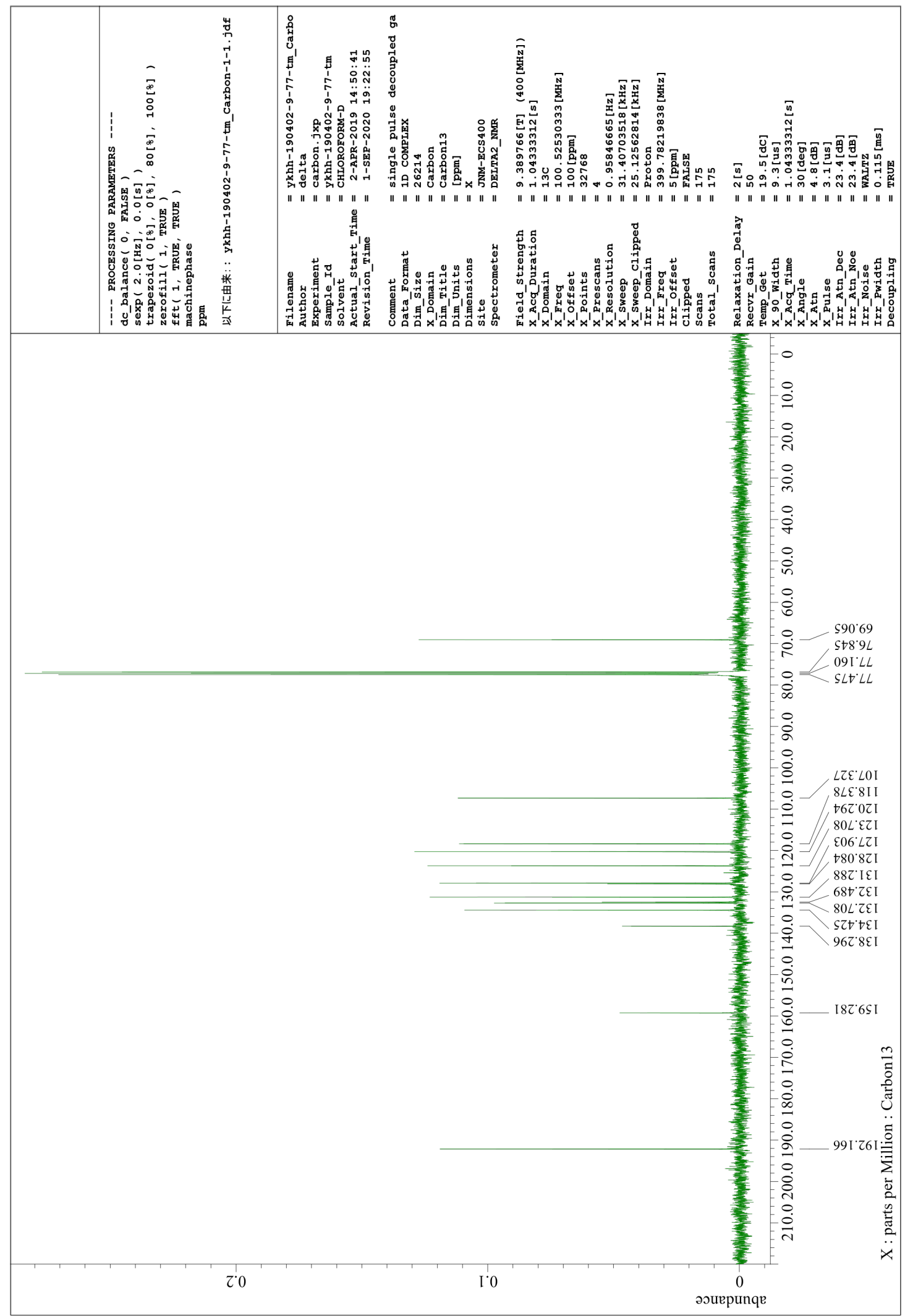


S2

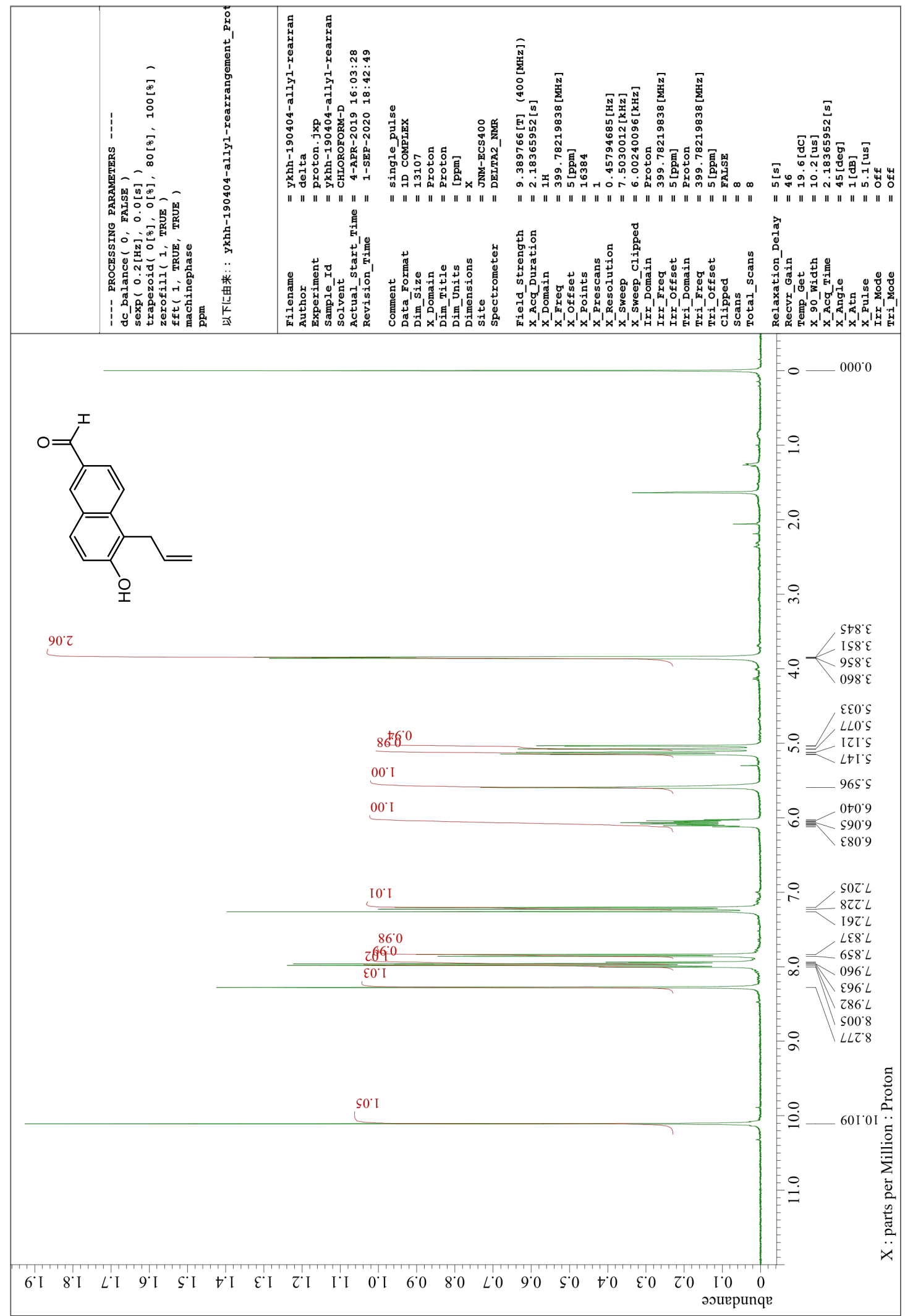




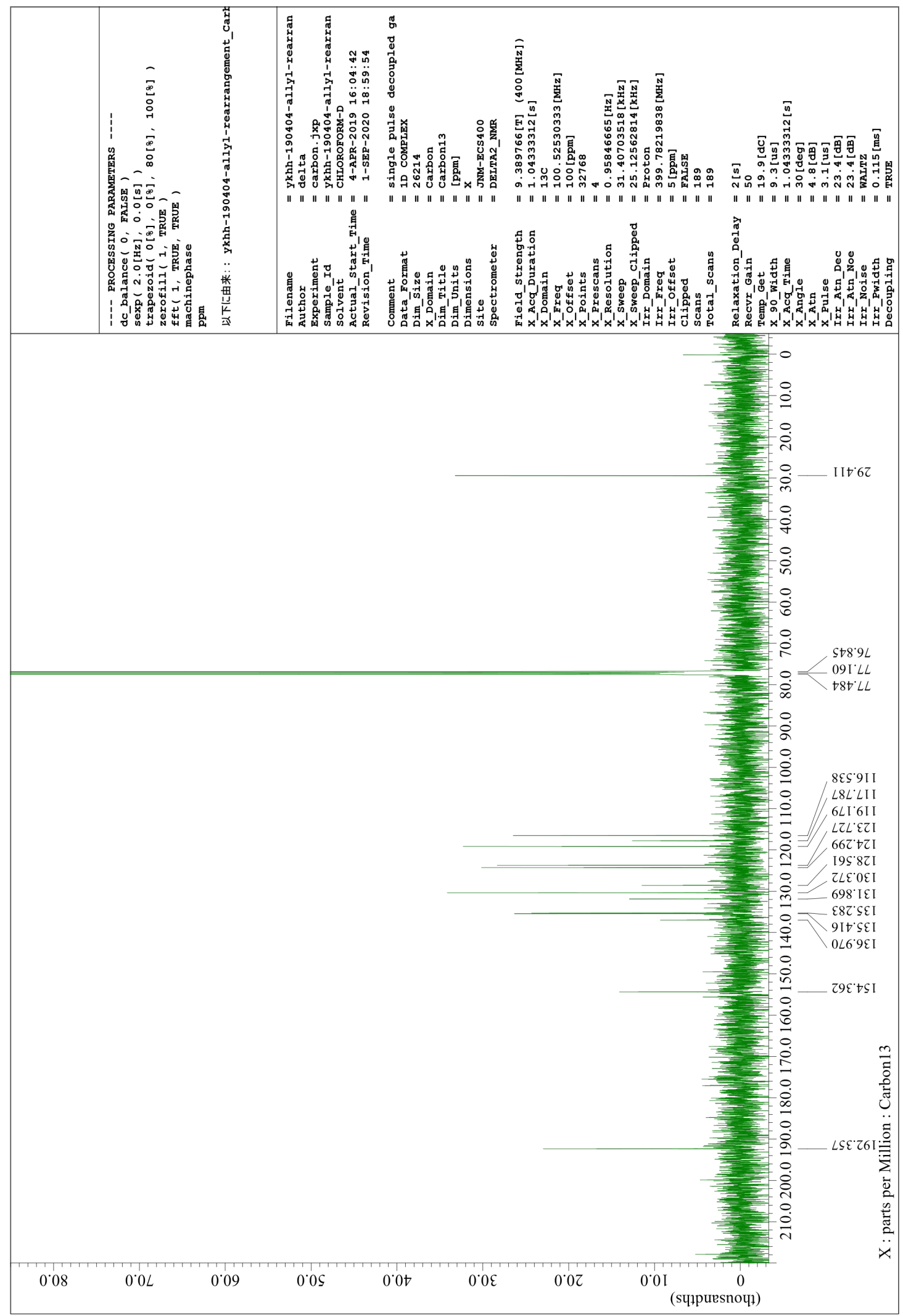


Supporting Information

S5

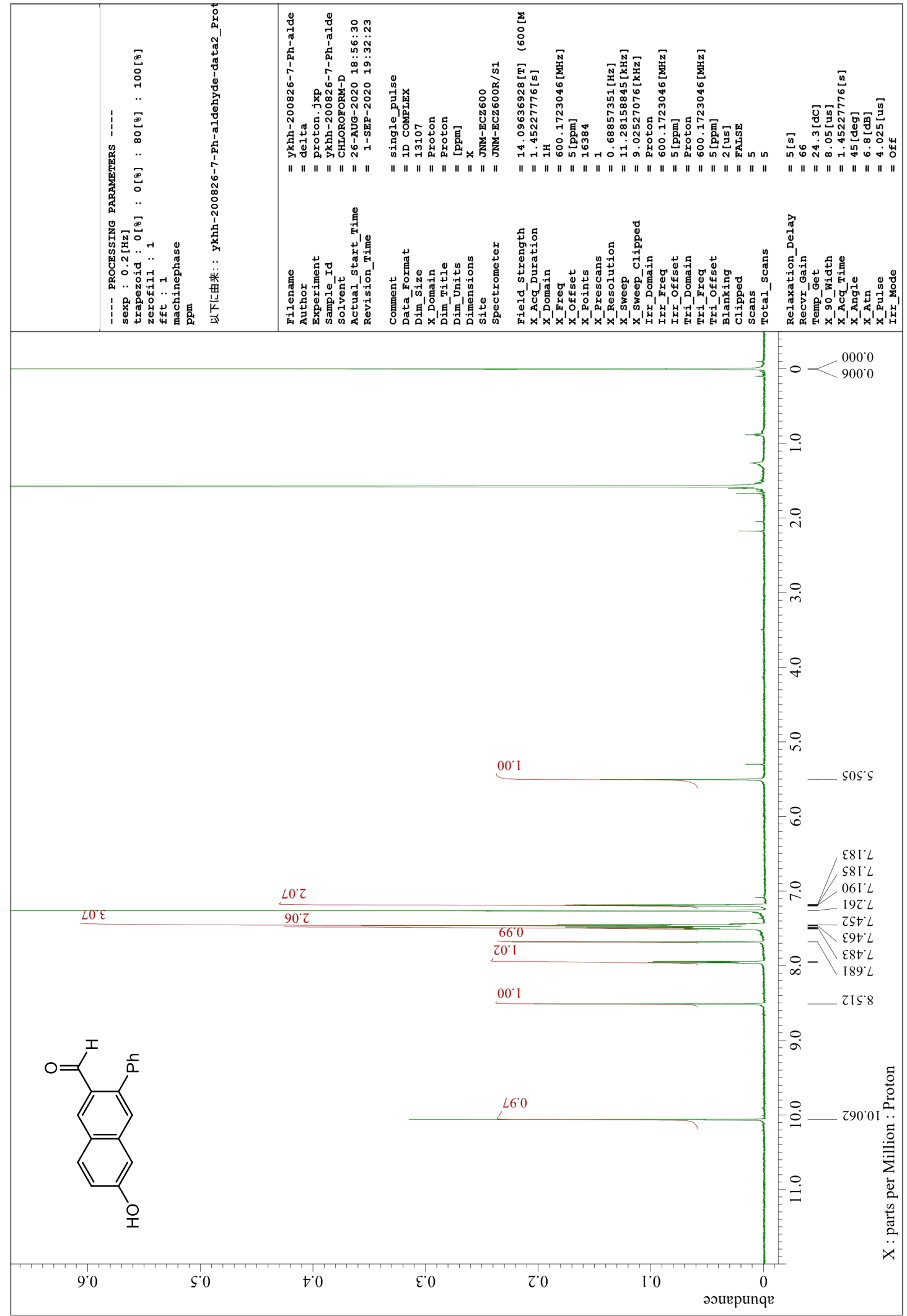




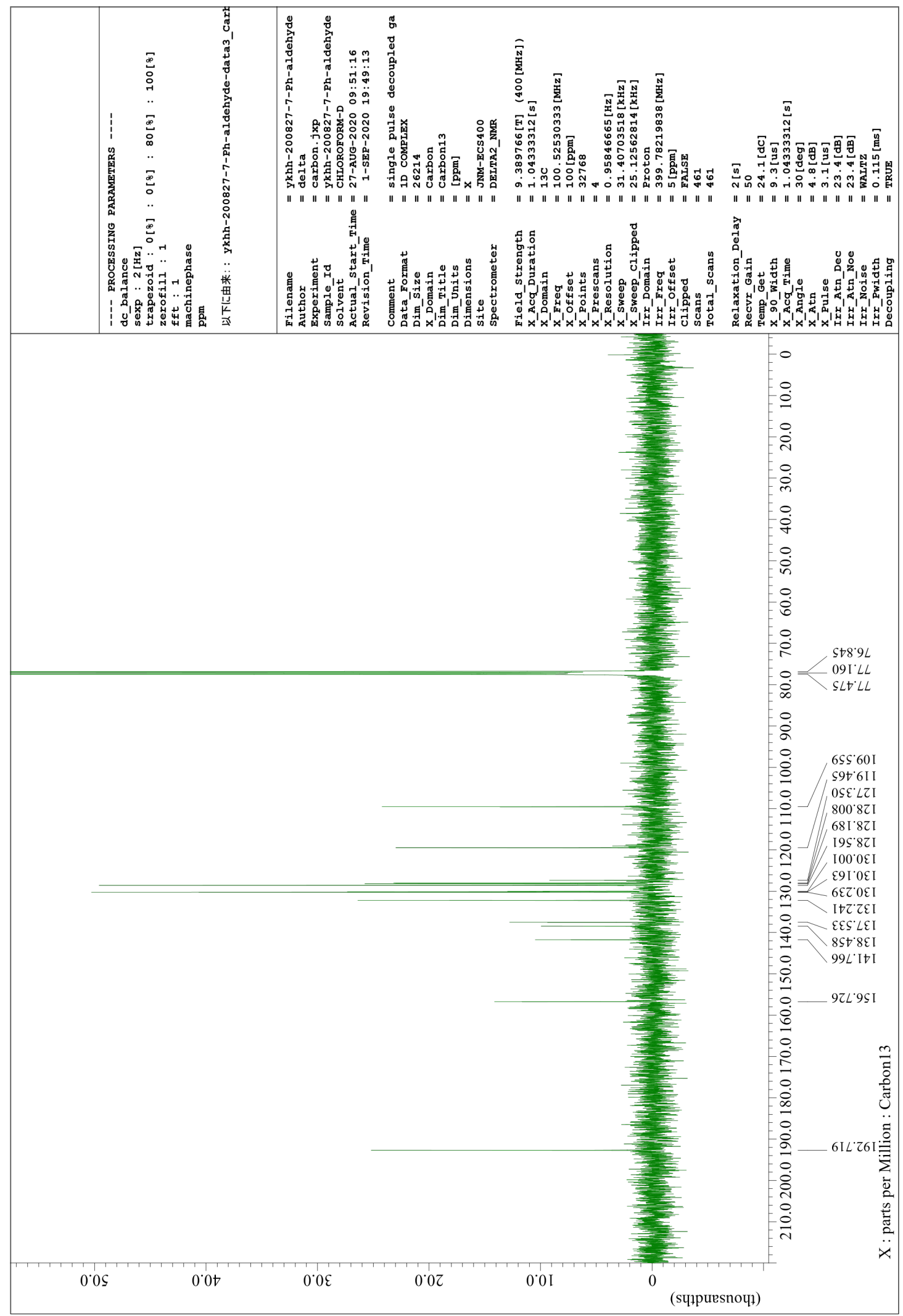


$1 b$

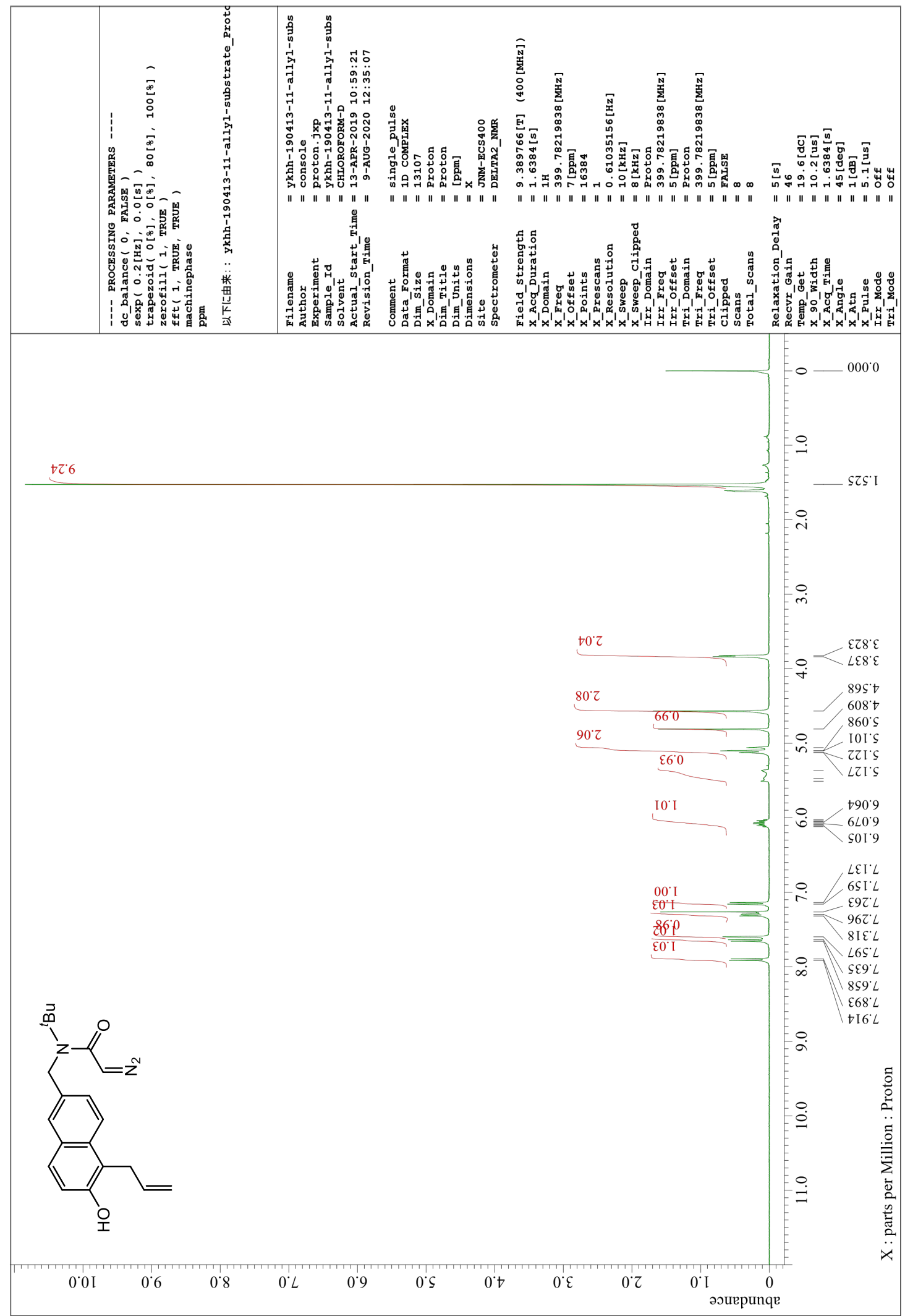




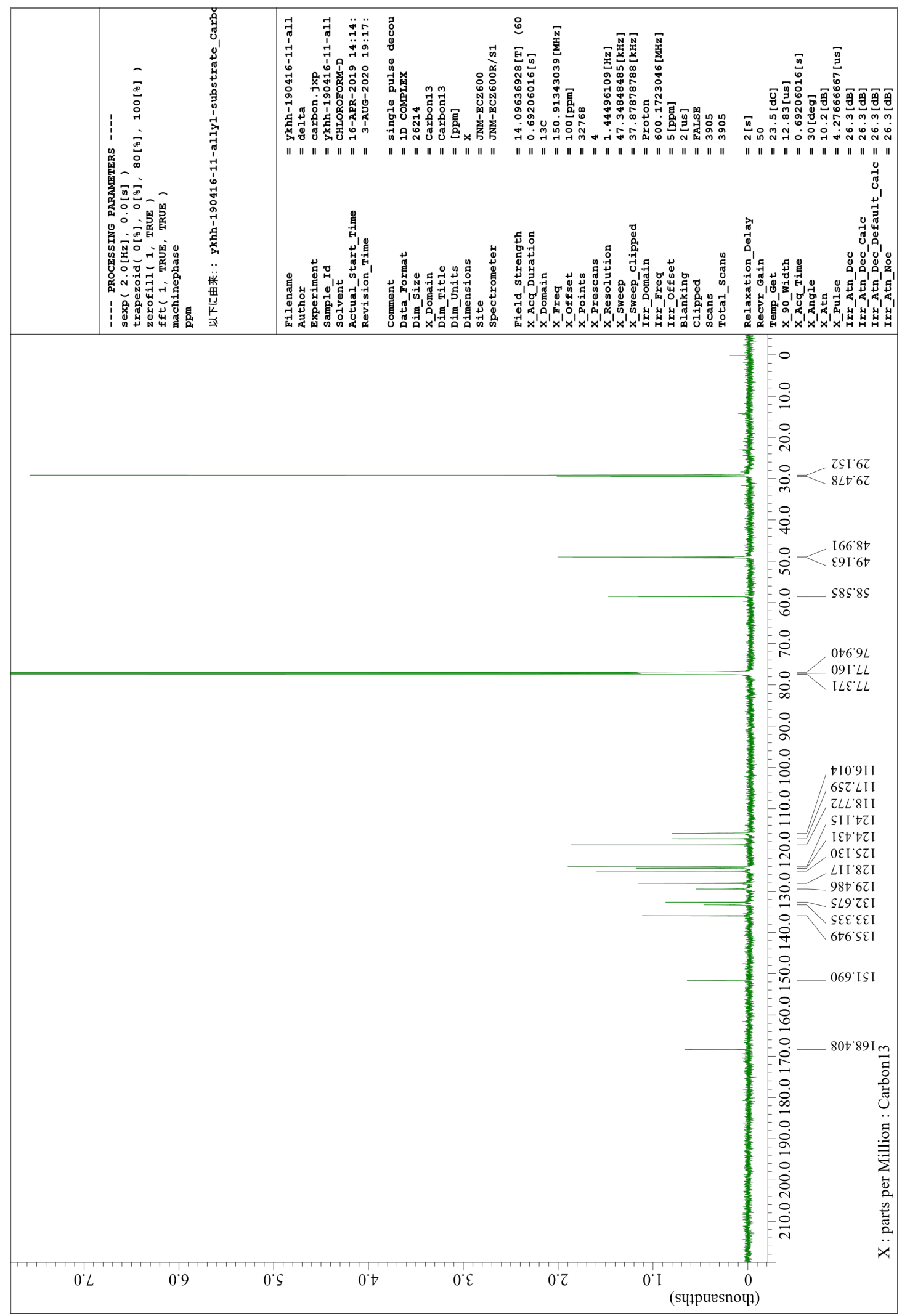


Supporting Information

$1 c$

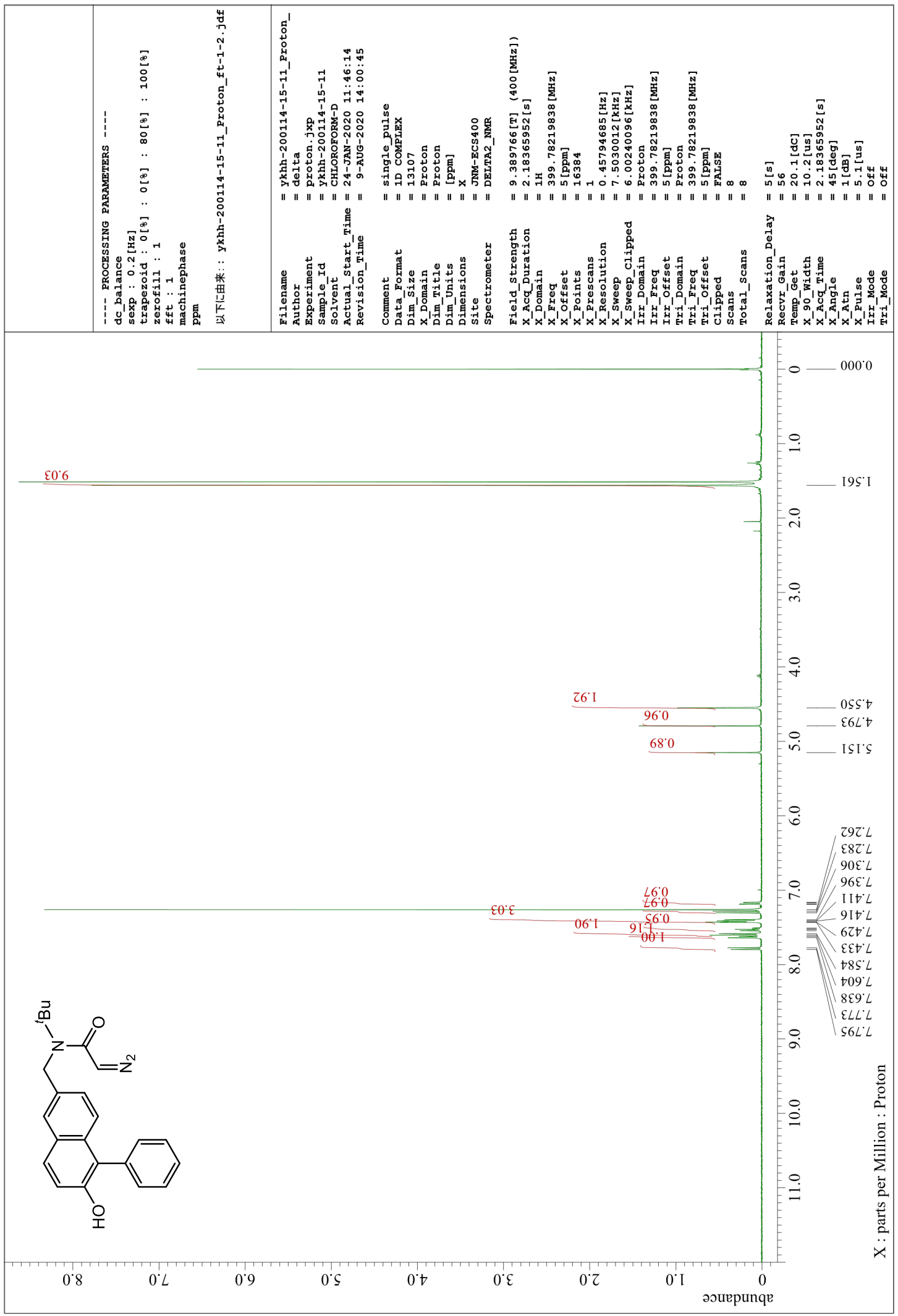


Supporting Information

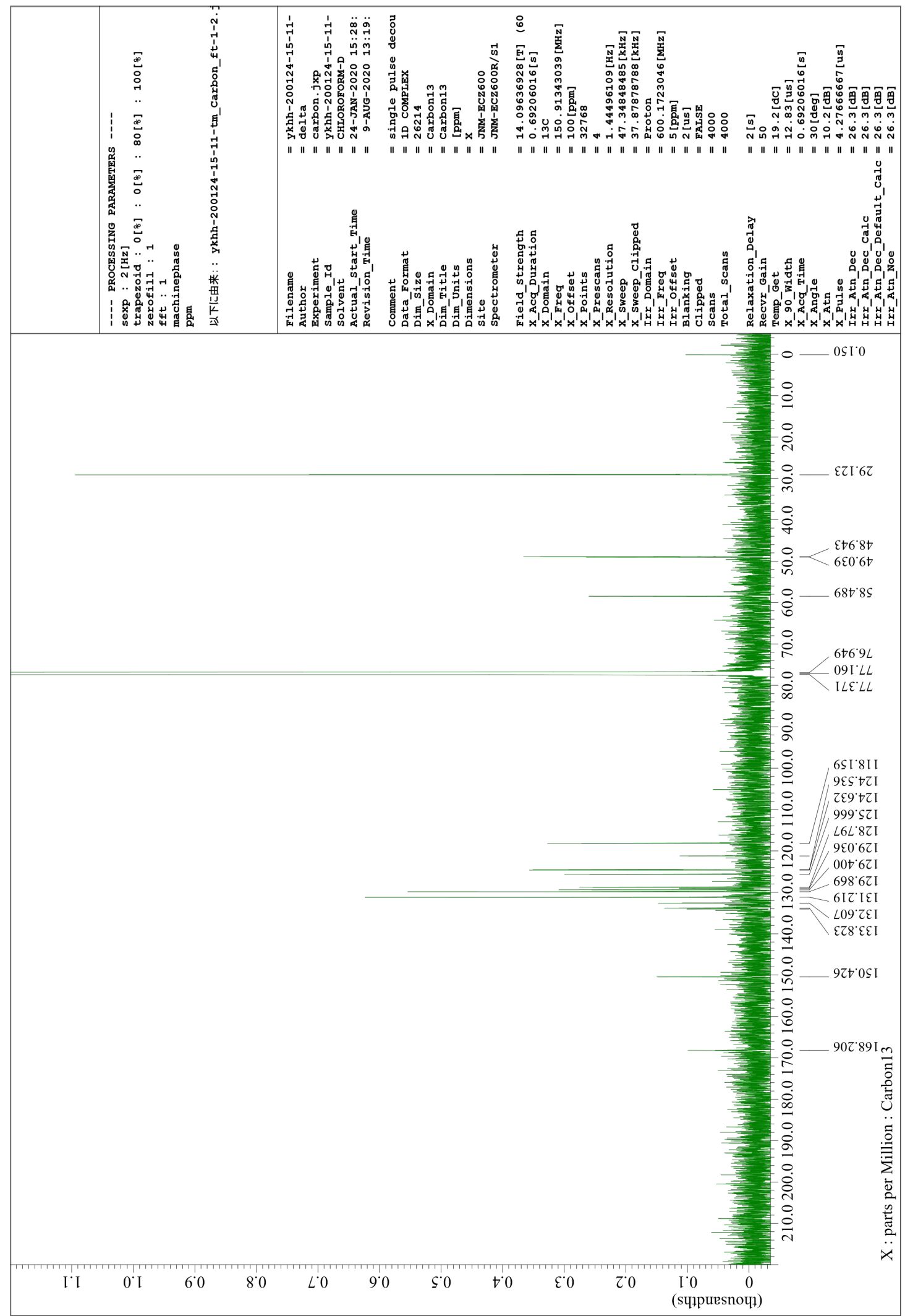


$1 d$

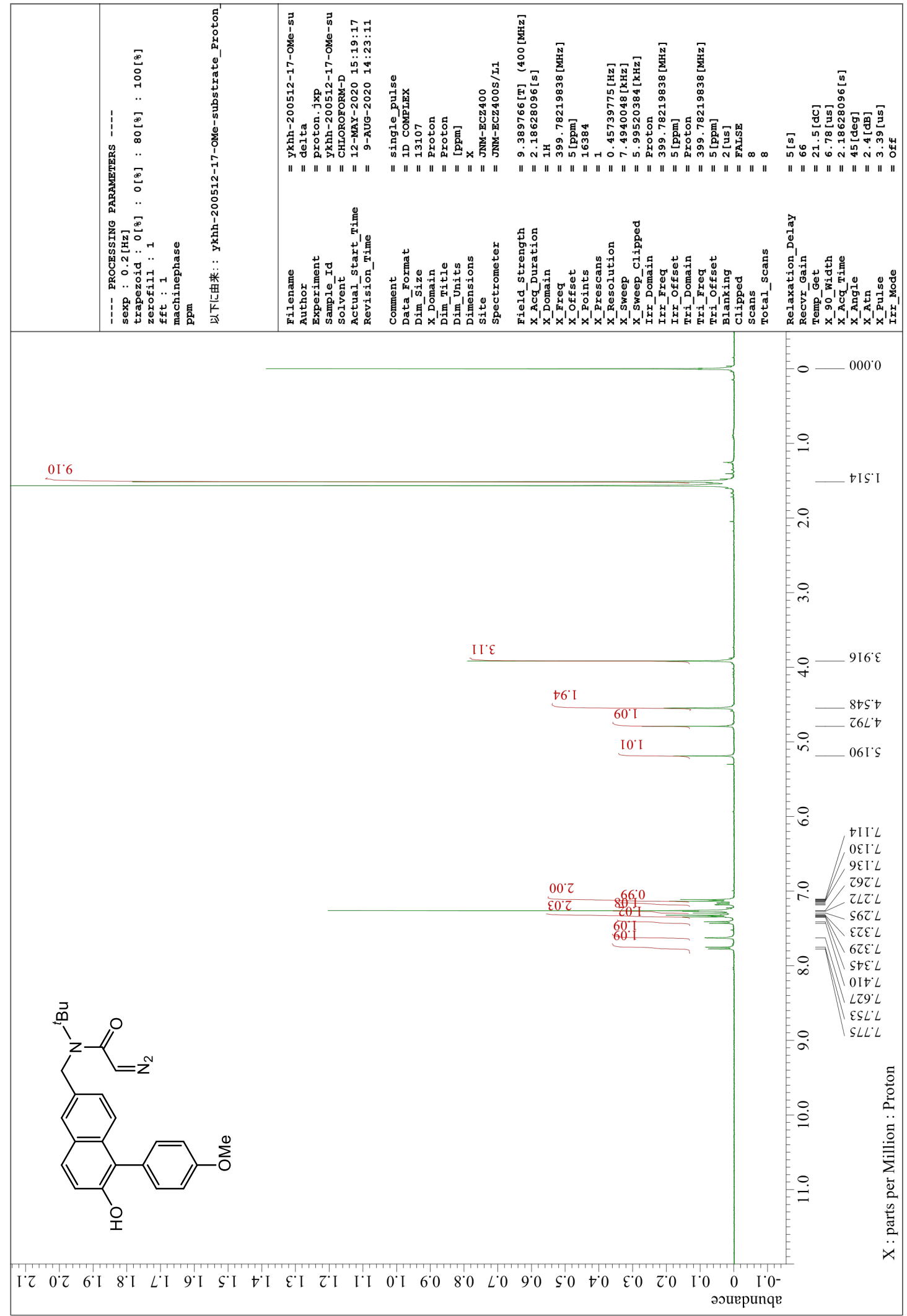




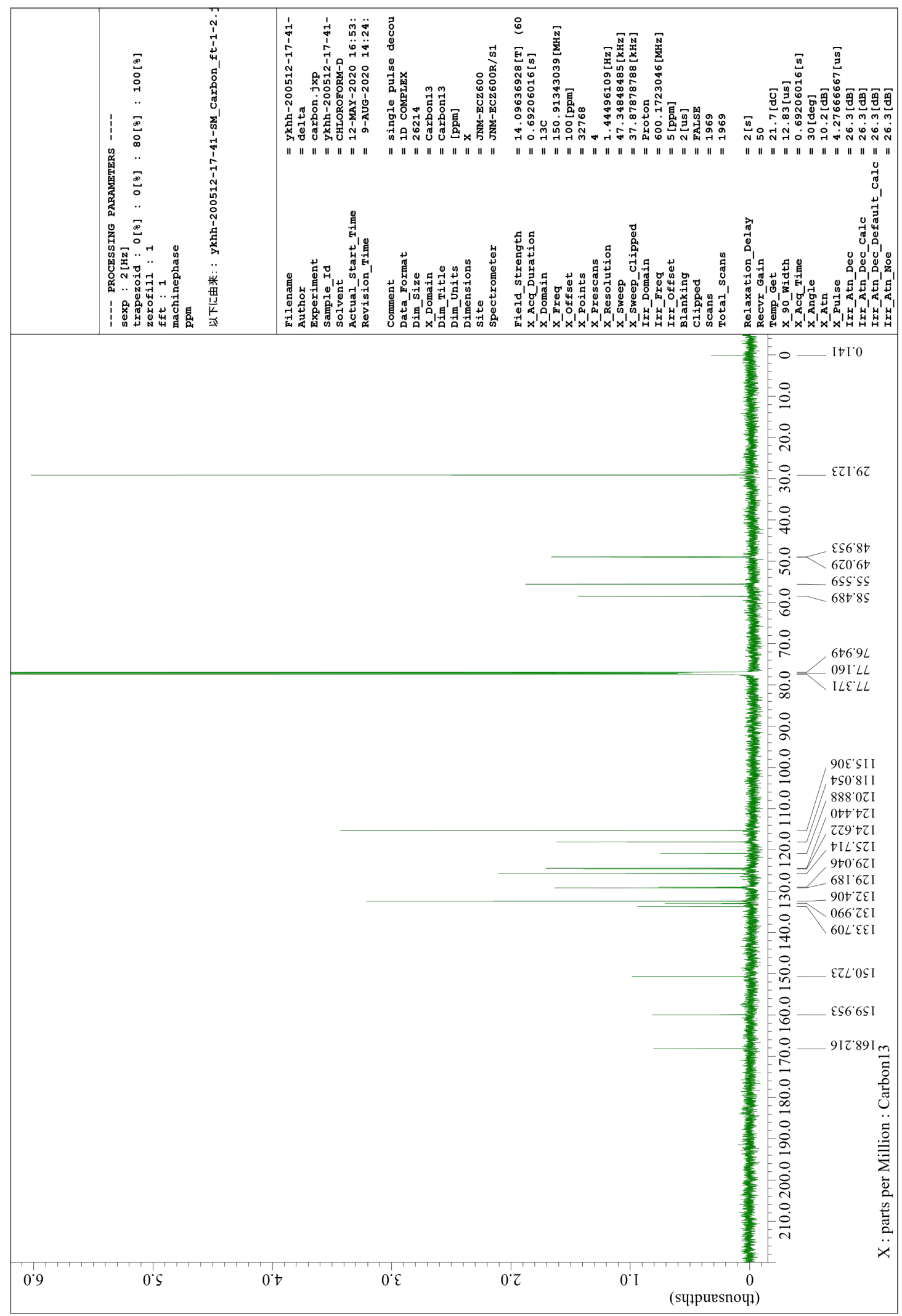


Supporting Information

$1 e$

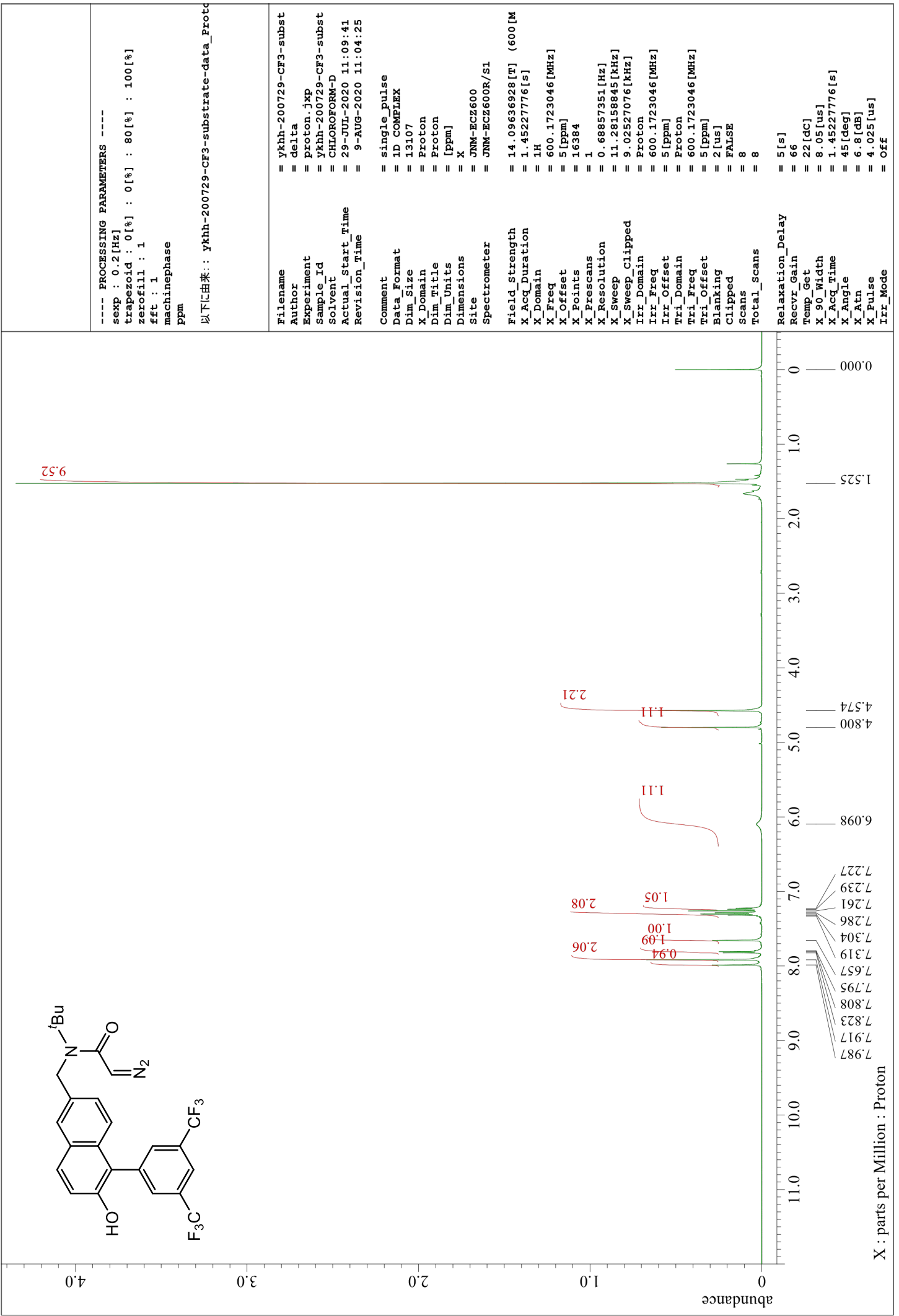




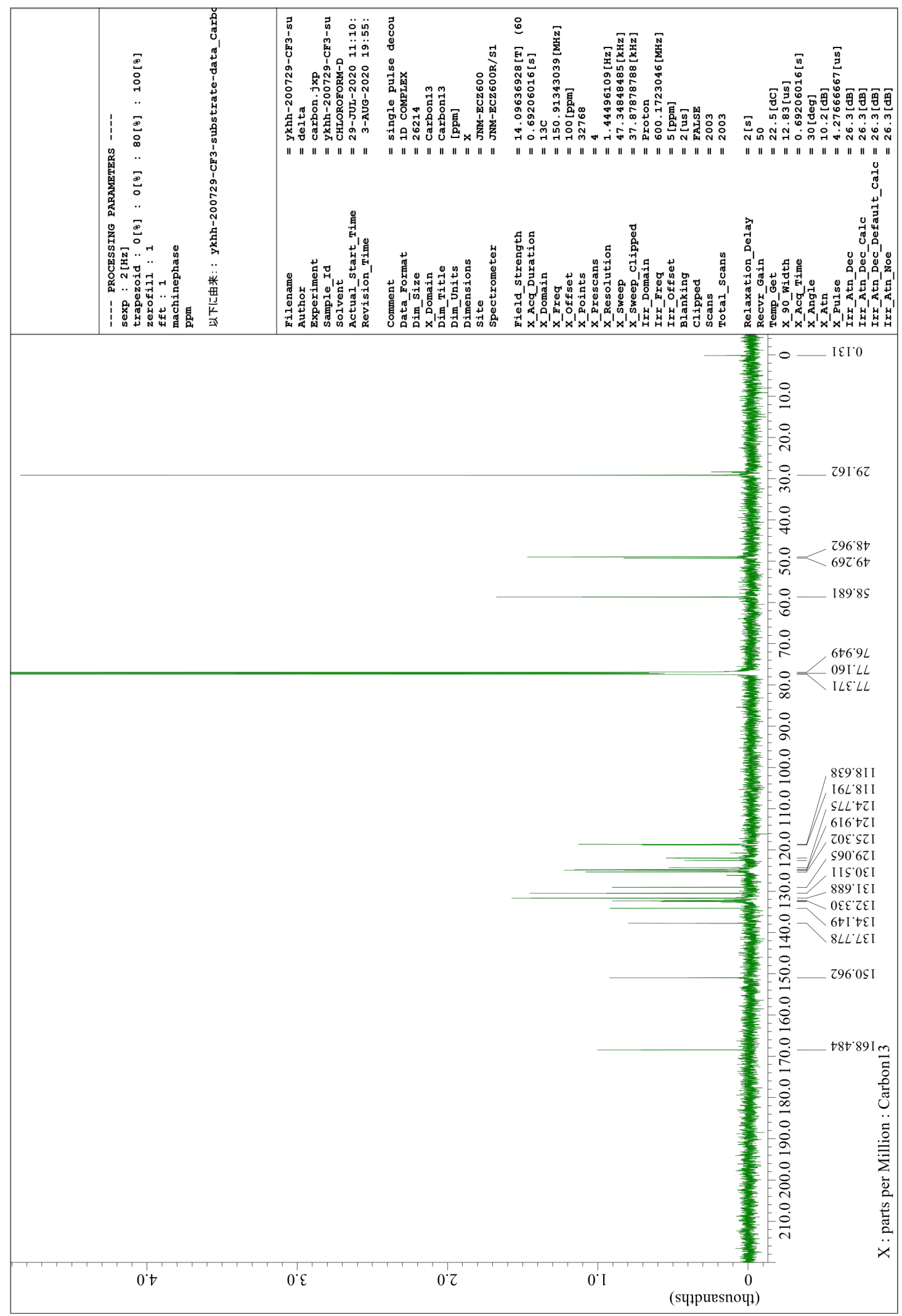




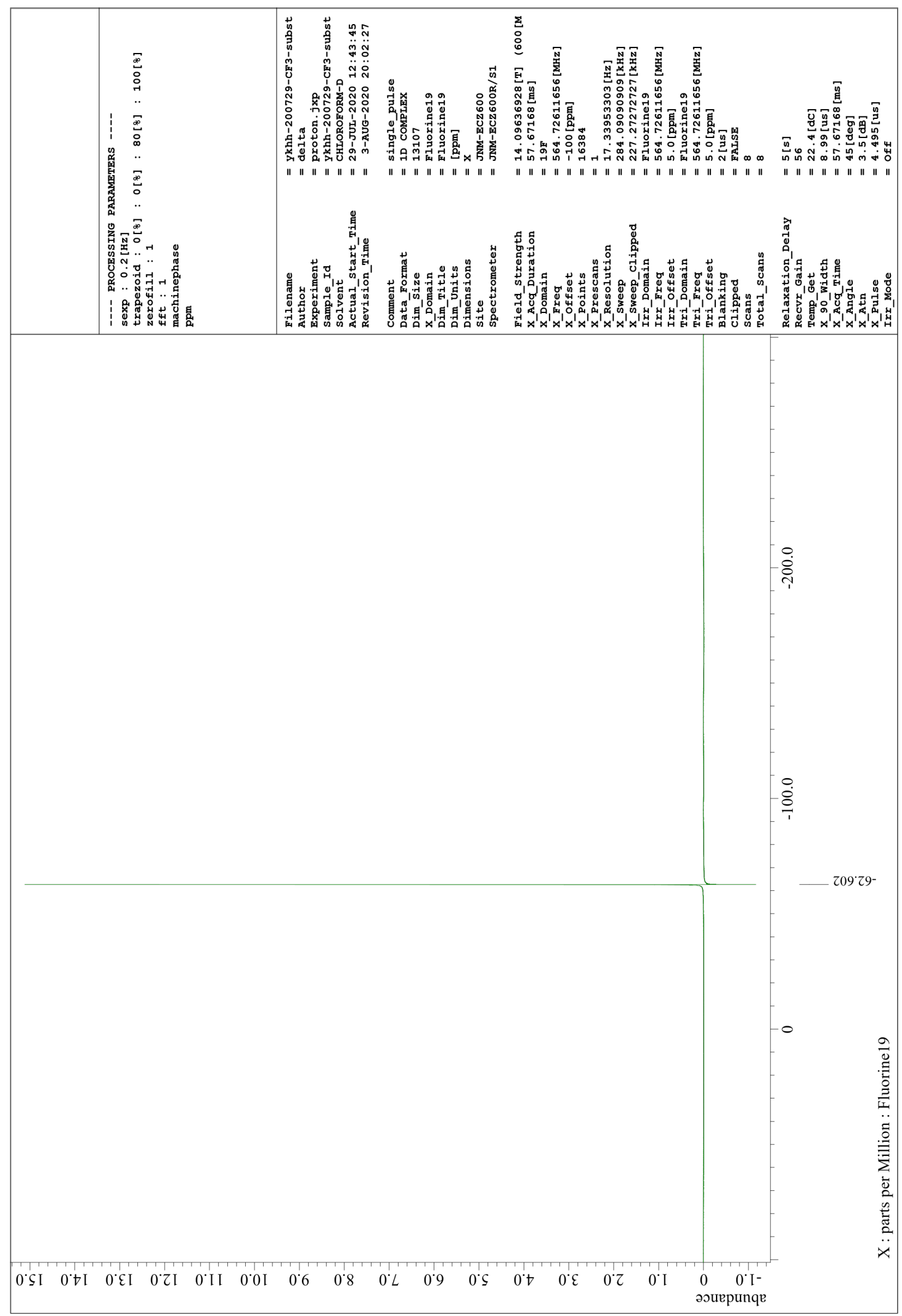


1f

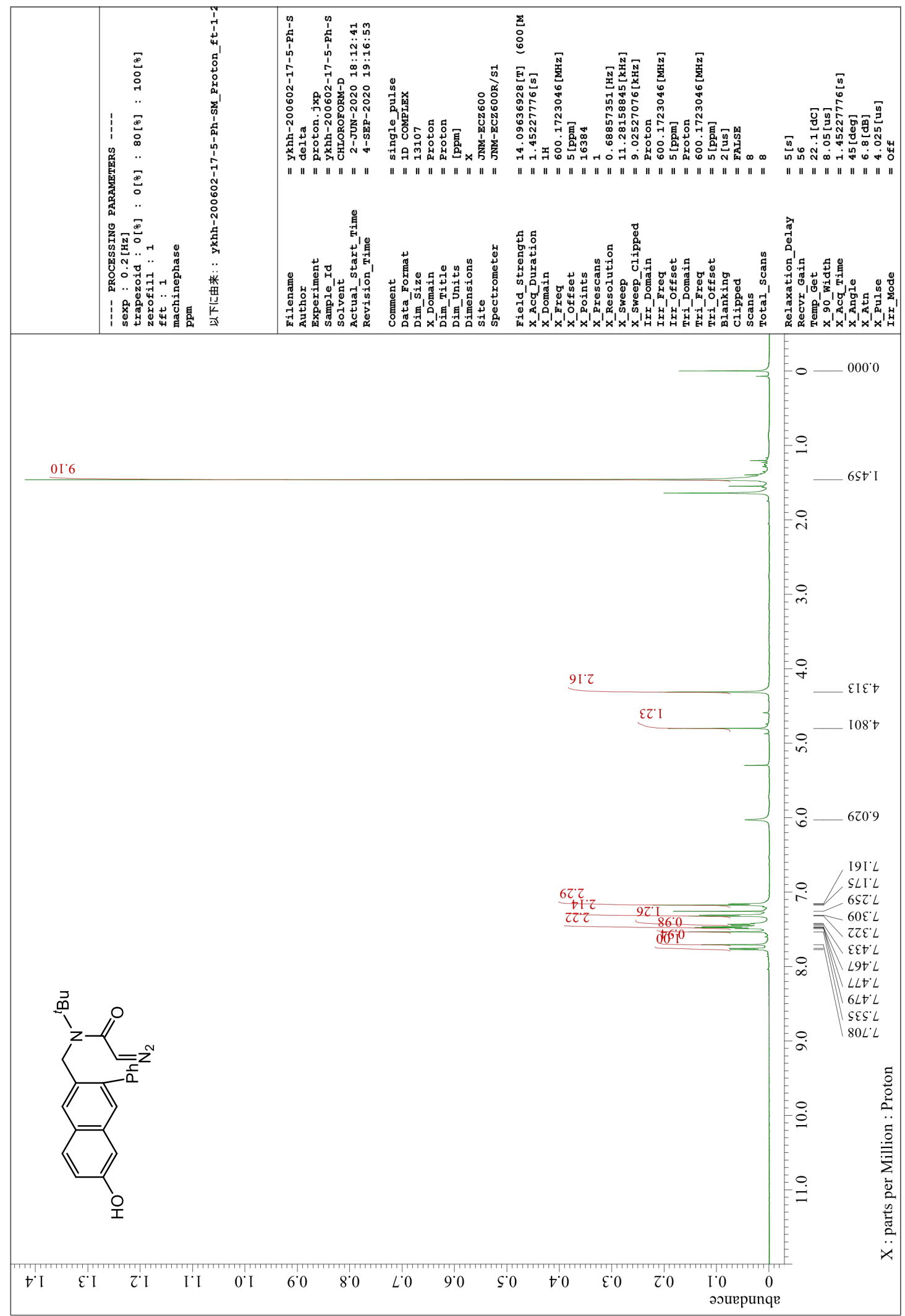




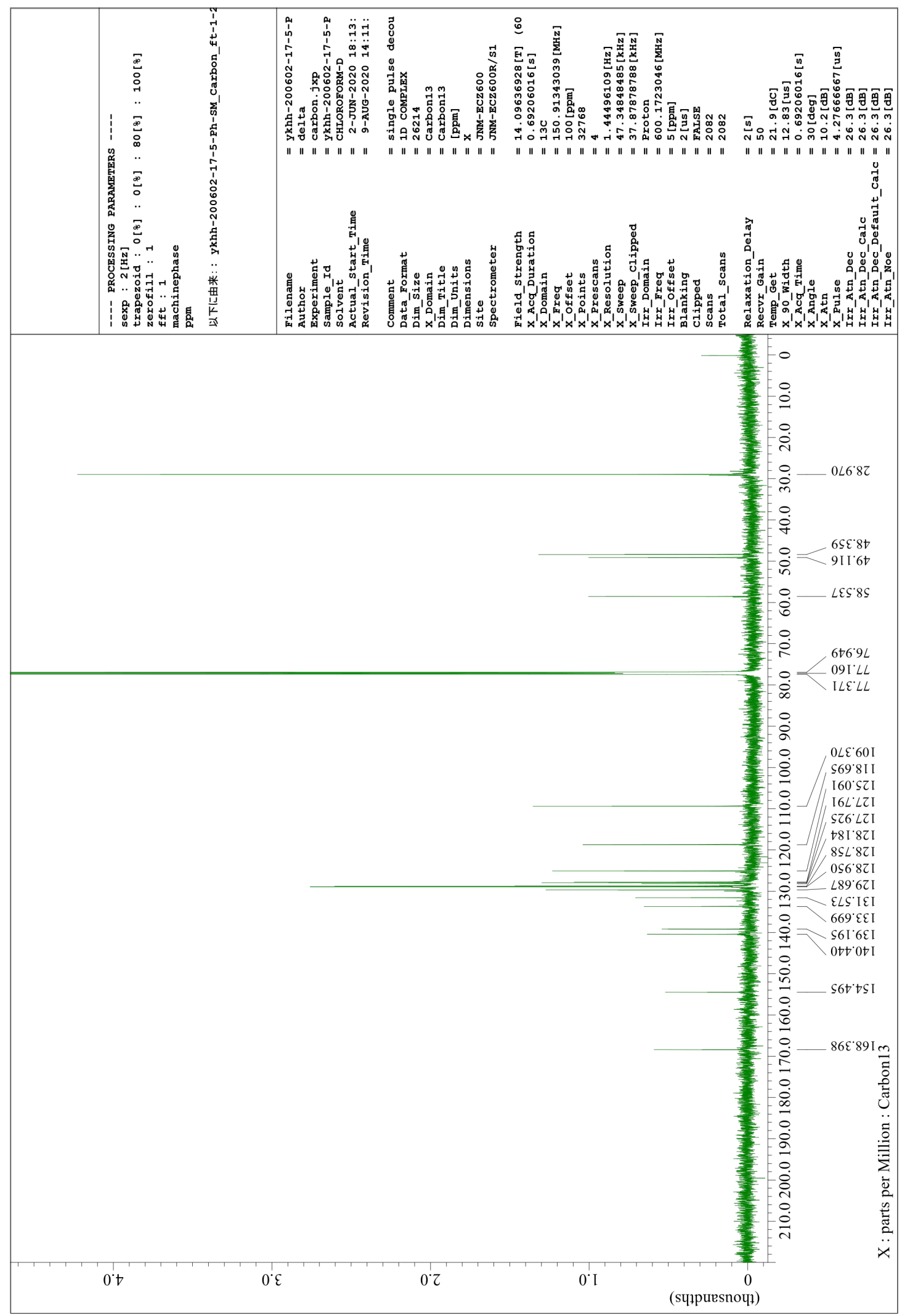


Supporting Information

$1 \mathrm{~g}$

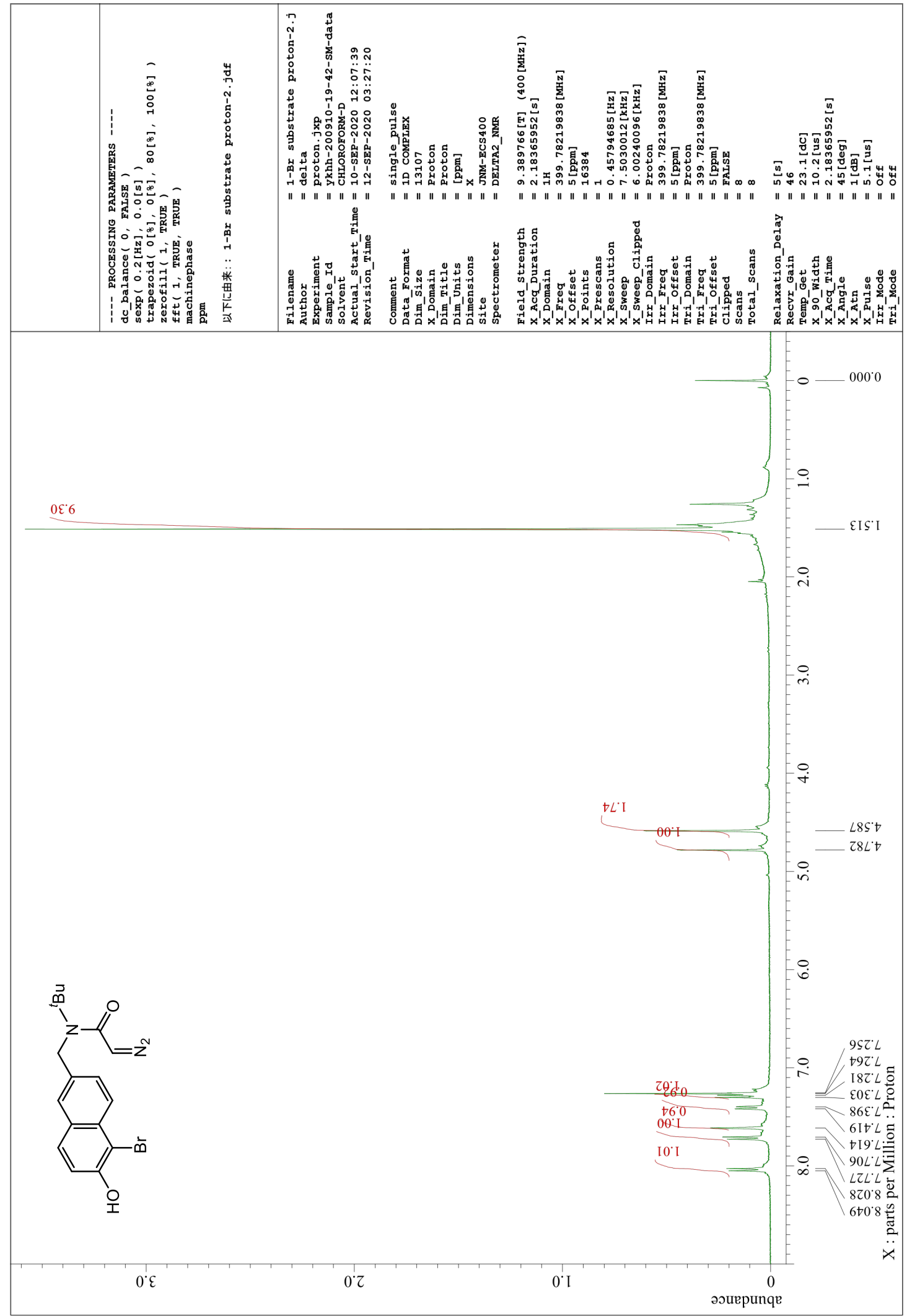




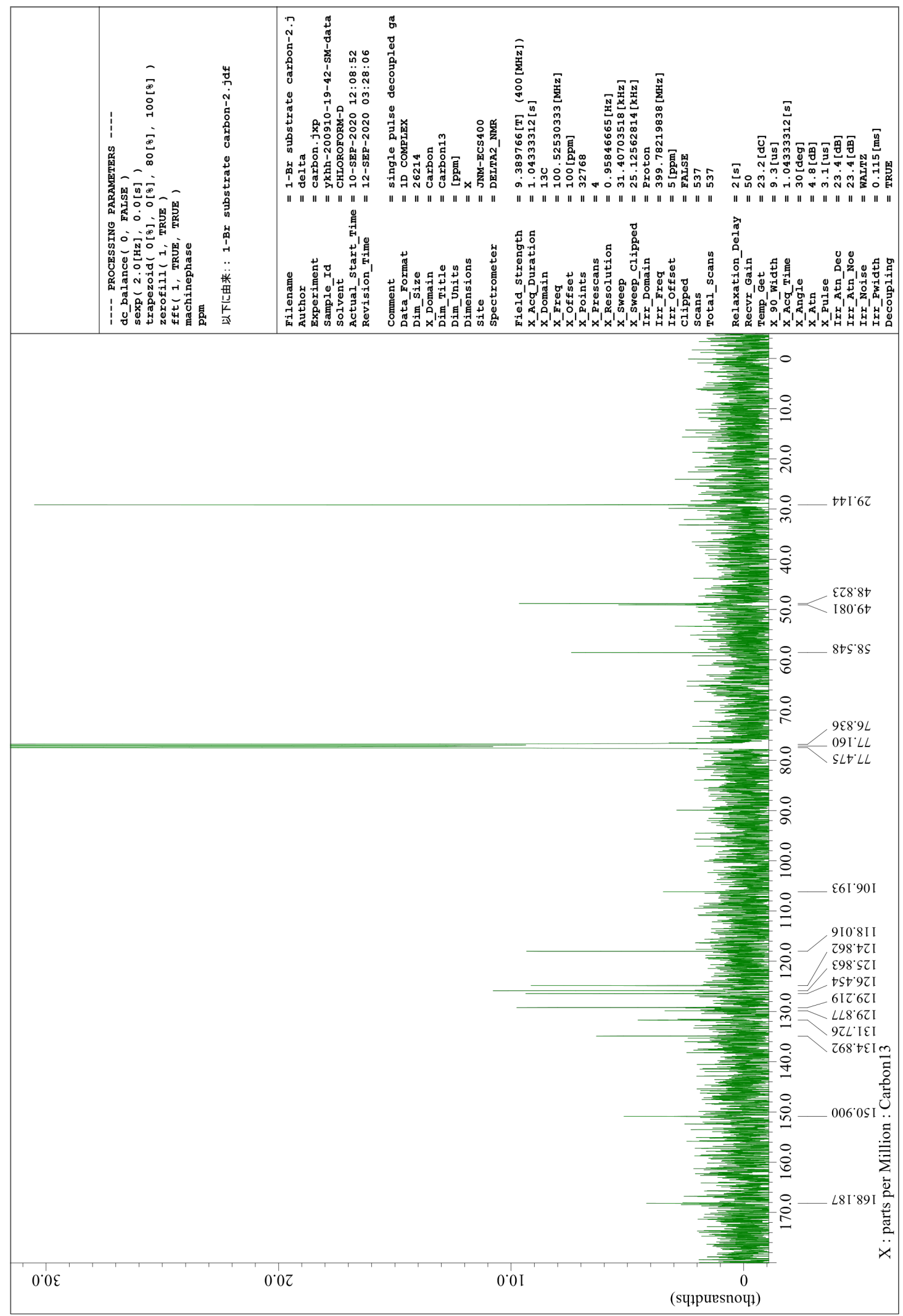


$1 \mathrm{~h}$

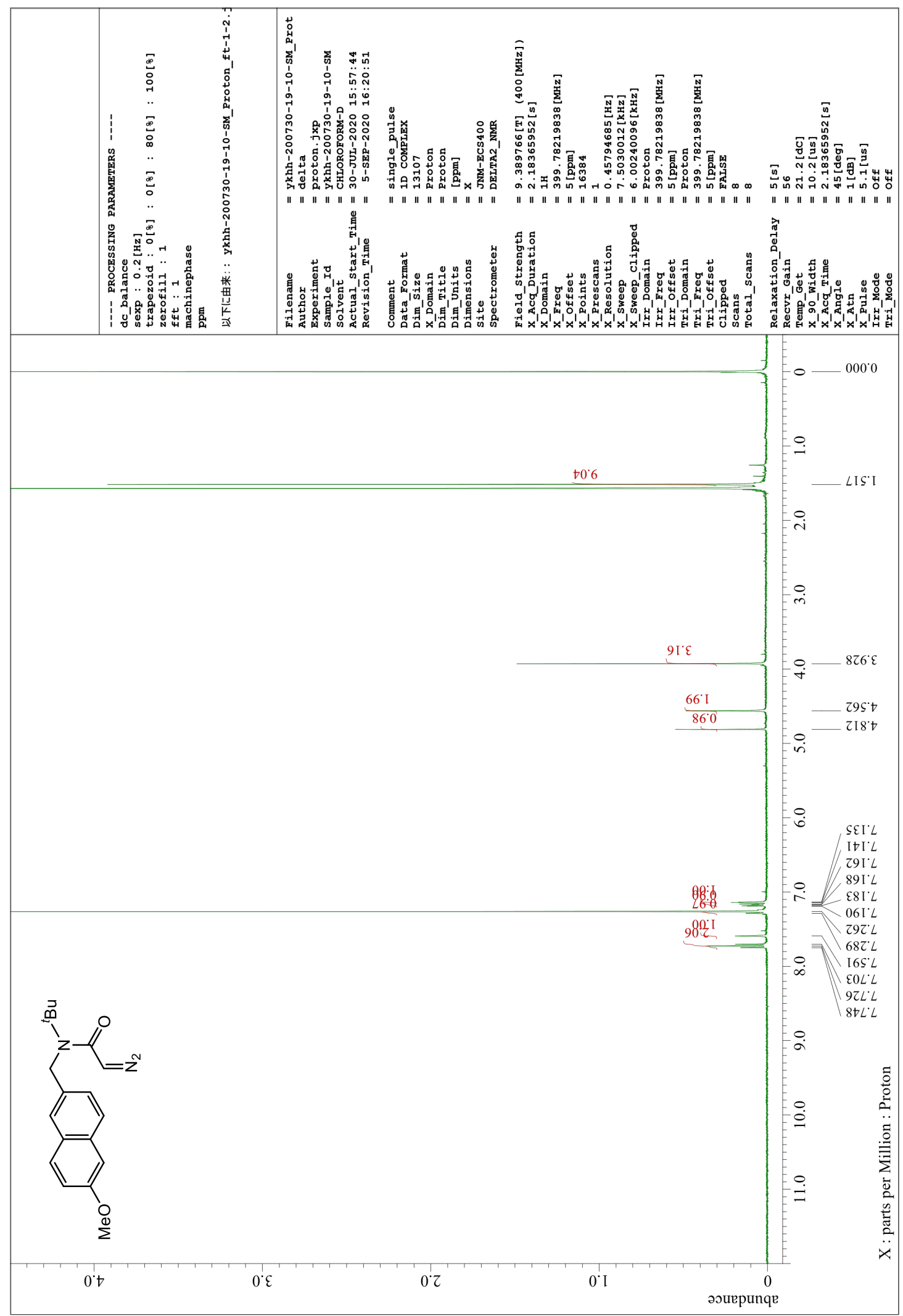




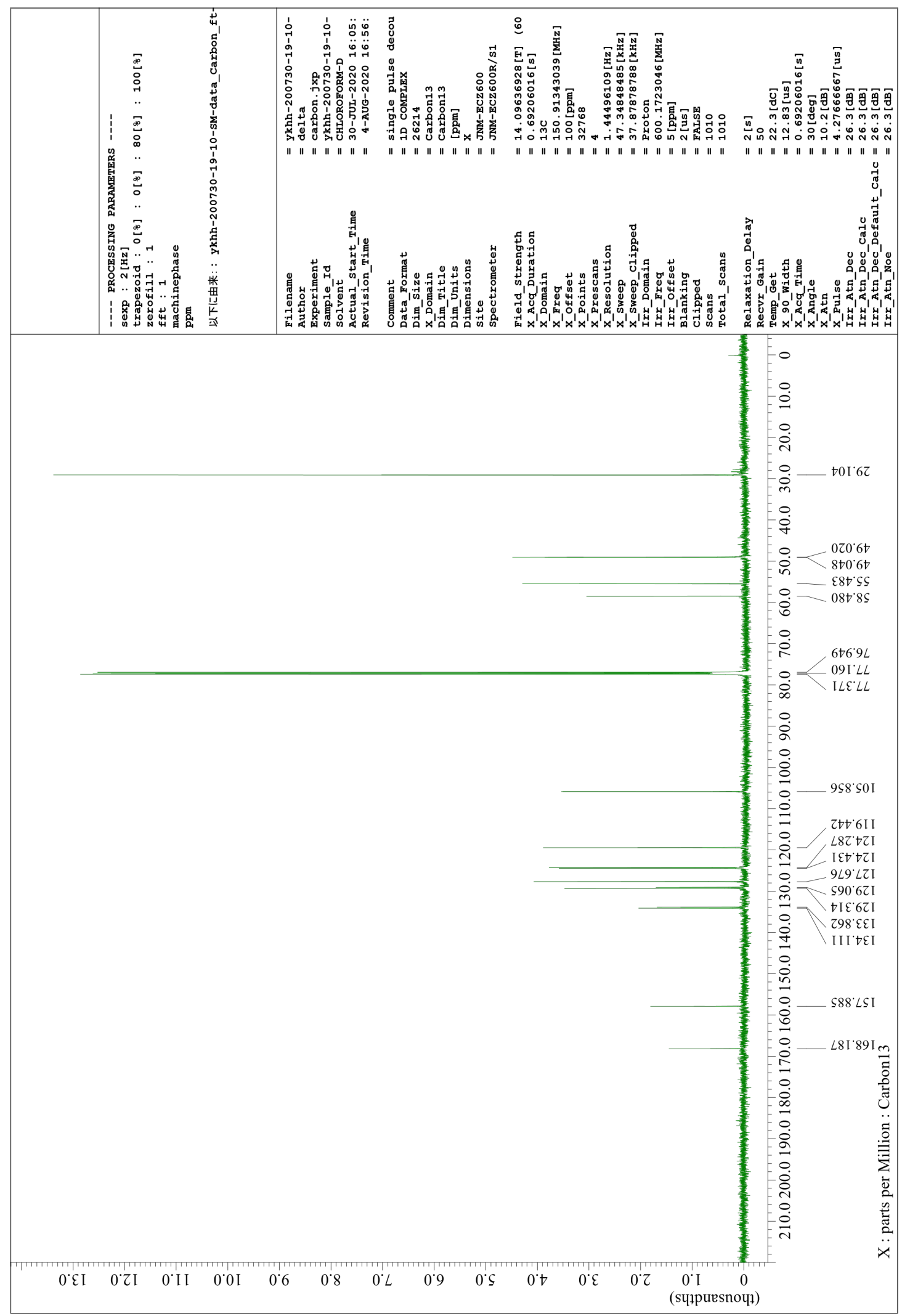


$1 \mathrm{i}$

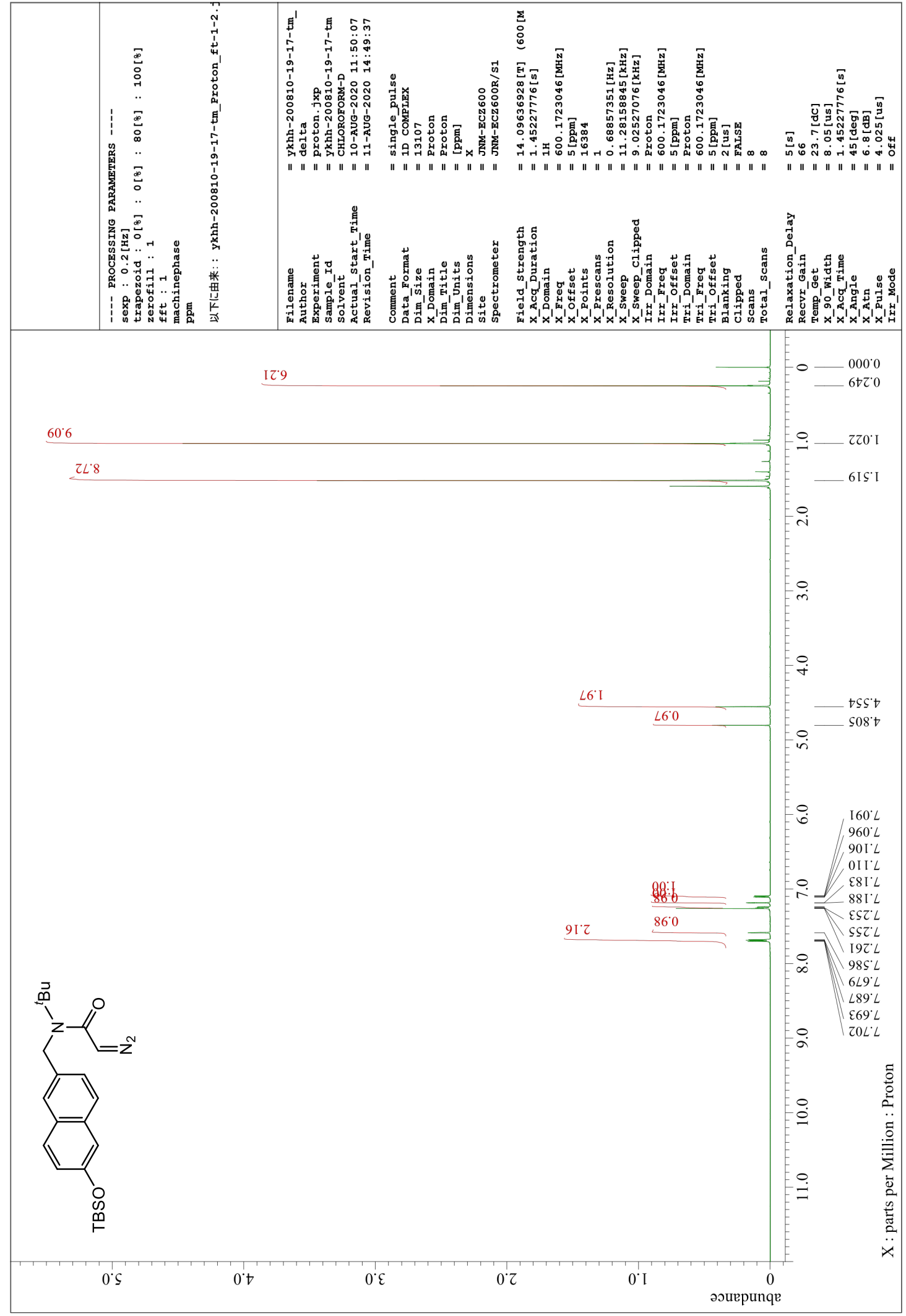




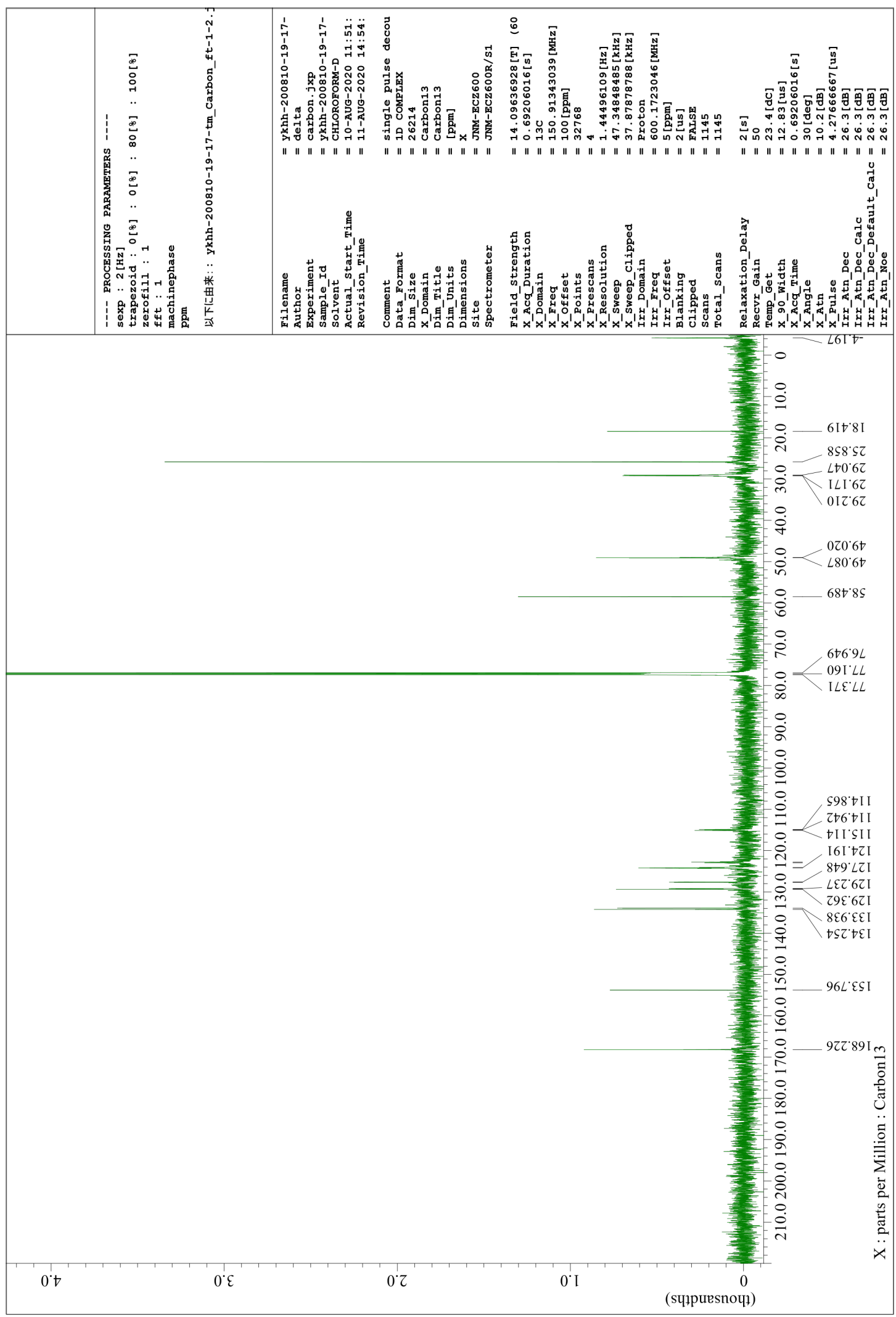




\section{References}

[1] Z. Zuo, H. Wang, L. Fan, J. Liu, Y. Wang, X. Luan, Angew. Chem. Int. Ed. 2017, 56, 2767-2771; Angew. Chem. 2017, 129, 2811-2815.

[2] L. Han, H. Wang, X. Luan, Org. Chem. Front. 2018, 5, 2453-2457.

[3] Gaussian 16, Revision A.03, M. J. Frisch, G. W. Trucks, H. B. Schlegel, G. E. Scuseria, M. A. Robb, J. R. Cheeseman, G. Scalmani, V. Barone, G. A. Petersson, H. Nakatsuji, X. Li, M. Caricato, A. V. Marenich, J. Bloino, B. G. Janesko, R. Gomperts, B. Mennucci, H. P. Hratchian, J. V. Ortiz, A. F. Izmaylov, J. L. Sonnenberg, D. Williams-Young, F. Ding, F. Lipparini, F. Egidi, J. Goings, B. Peng, A. Petrone, T. Henderson, D. Ranasinghe, V. G. Zakrzewski, J. Gao, N. Rega, G. Zheng, W. Liang, M. Hada, M. Ehara, K. Toyota, R. Fukuda, J. Hasegawa, M. Ishida, T. Nakajima, Y. Honda, O. Kitao, H. Nakai, T. Vreven, K. Throssell, J. A. Montgomery, Jr., J. E. Peralta, F. Ogliaro, M. J. Bearpark, J. J. Heyd, E. N. Brothers, K. N. Kudin, V. N. Staroverov, T. A. Keith, R. Kobayashi, J. Normand, K. Raghavachari, A. P. Rendell, J. C. Burant, Supporting Information S123 S. S. Iyengar, J. Tomasi, M. Cossi, J. M. Millam, M. Klene, C. Adamo, R. Cammi, J. W. Ochterski, R. L. Martin, K. Morokuma, O. Farkas, J. B. Foresman, and D. J. Fox, Gaussian, Inc., Wallingford CT, 2016.

[4] (a) A. D. Beche, Phys. Rev. 1988, A38, 3098-3100. (b) A. D. Beche, J. Chem. Phys. 1993, 98. 1372-1377. (c) A. D. Beche, J. Chem. Phys. 1993, 98, 5648-5652. (d) C. Lee, W. Yang, R. G. Parr, Phys. Rev. 1988, B37, 785-788.

[5] (a) K. Fukui, Acc. Chem. Res. 1981, 14, 363-368. (b) K. Ishida, K. Morokuma, A. Komornicki, J. Chem. Phys. 1977, 66, 2153-2156. (c) C. Gonzalez, H. B. Schlegel, J. Chem. Phys. 1989, 90, 2154-2161. (d) C. Gonzalez, H. B. Schlegel, J. Phys. Chem. 1990, 94, 5523-5527.

[6] SDD: D. Andrae, U. Häußermann, M. Dolg, H. Stoll, H. H. Preuß, Energy Adjusted ab initio Pseudopotentials for the Second and Third Row Transition Elements. Theoret. Chim. Acta 1990, 77, 123-141. 